\title{
Behavioural problems in dementia Caregiver issues
}


(c) M.E. de Vugt, Maastricht 2004

Cover design / layout

Production

ISBN
A. Weisscher

Ponsen \& Looijen BV, Wageningen

90-75579-19-5

Neuropsych Publishers is a nonprofit organisation, which aims at promoting the science of 'Brain and Behaviour' and improving the application of the products of this science in health care and education. Neuropsych Publishers accomplishes these aims by publishing books, dissertations and other products of scientific activity, by disseminating educational materials and publication of tests, assessment scales and other psychometric instruments in the field of Neuropsychology, Neuropsychiatry and other areas within the domain of Brain and Behaviour.

Postal address:

Neuropsych Publishers

Department of Psychiatry and Neuropsychiatry

Maastricht University

P.O. Box 616

NL-6200 MD Maastricht

The Netherlands

www-np.unimaas.nl 


\title{
Behavioural problems in dementia Caregiver issues
}

\author{
PROEFSCHRIFT \\ ter verkrijging van de graad van doctor aan de Universiteit Maastricht, \\ op gezag van de Rector Magnificus, Prof. mr. G.P.M.F. Mols, \\ volgens het besluit van het college van Decanen, in het openbaar te verdedigen \\ op woensdag 15 september 2004 om 16.00 uur
}

door

Marjolein Elisabeth de Vugt 


\section{Promotores}

Prof. dr. F.R.J. Verhey

Prof. dr. J. Jolles

\section{Co-promotor}

Dr. F. Stevens

\section{Beoordelingscommissie}

Prof. dr. R. Kempen (voorzitter)

Dr. N.A. Nicolson

Prof. dr. J. van Os

Prof. dr. J.M.G.A. Schols (Universiteit van Tilburg)

Dr. M. Vernooy-Dassen (Katholieke Universiteit Nijmegen)

The research described in this thesis was performed in the Maastricht Brain \& Behaviour Institute, and the department of Psychiatry and Neuropsychology, Maastricht University, Maastricht, the Netherlands, in collaboration with the Academic Hospital Maastricht and the geriatric division of the Regional Institute for Community Mental Health (RIAGG), Maastricht.

The Netherlands Organisation for Scientific Research (NWO; grant 940-33-039) financially supported the research described in this thesis.

Deloitte., Novartis Neuroscience, Lundbeck BV, Janssen-Cilag BV, Sigma Tau Ethifarma BV, Alzheimer Nederland and Alzheimer Afdeling stadsgewest Breda financially supported the publication of this thesis. 


\section{Contents}

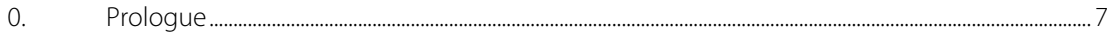

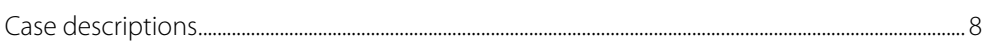

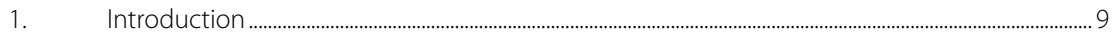

Behavioural problems in dementia ......................................................................................................... 10

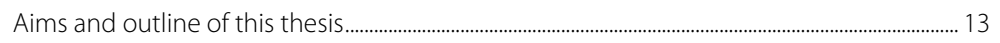

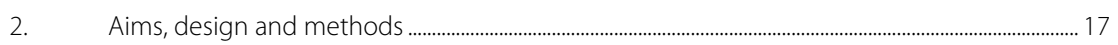

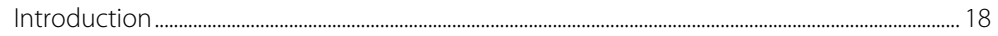

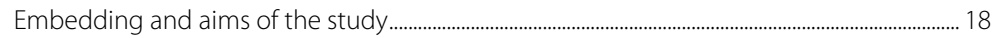

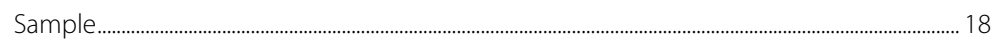

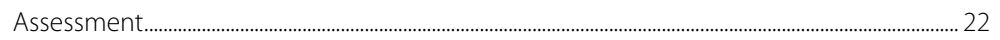

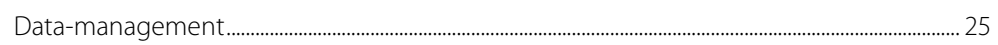

3. Factor analysis of the Neuropsychiatric Inventory (NPI) ................................................................ 27

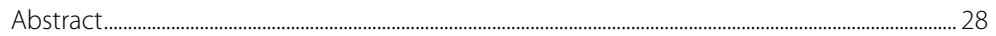

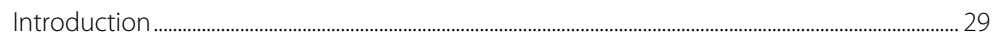

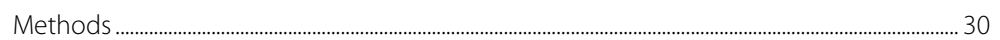

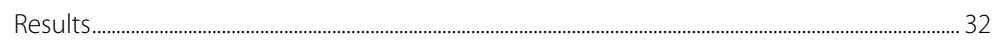

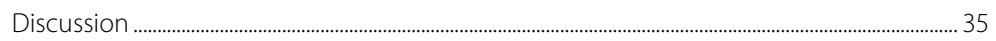

4. Salivary cortisol patterns in caregivers ......................................................................................................... 41

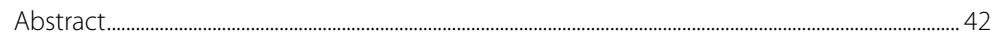

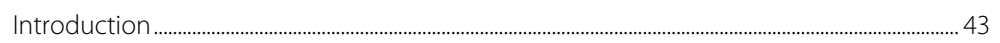

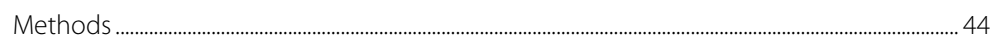

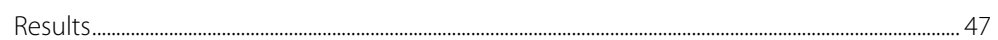

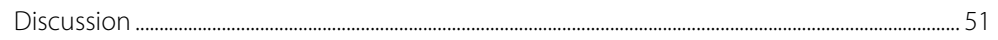

5. Behavioural disturbances in dementia patients and quality of the marital relationship ....... 55

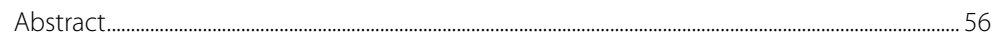

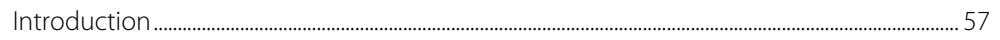

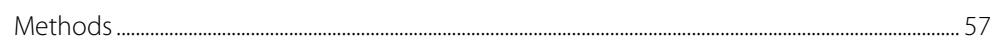

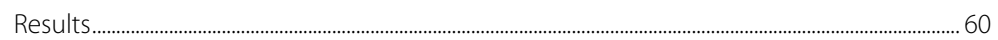

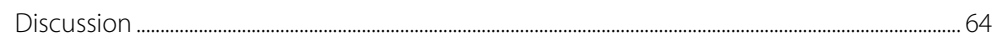

6. Impact of behavioural problems on spousal caregivers: a comparison between Alzheimer disease and Frontotemporal dementia ......................................................................................................... 67

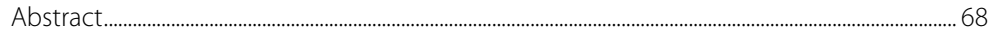

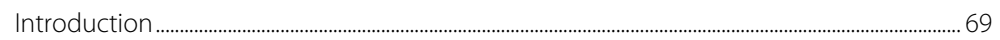

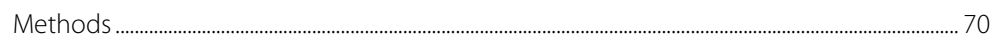

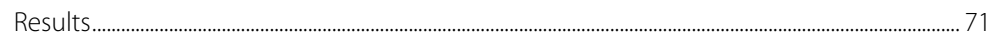

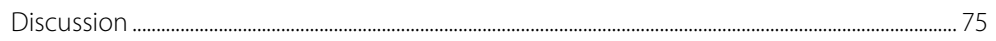

7. The effects of behavioural symptoms on institutionalisation in dementia .................................. 79

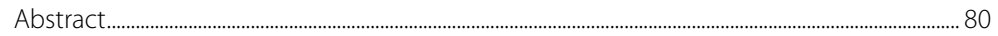

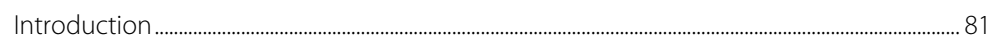


Methods

Results.

Discussion

. .88

8. Influence of caregiver management strategies on patient behaviour in dementia................91

Abstract

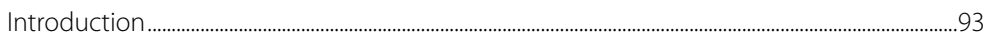

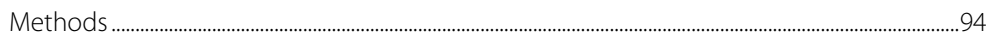

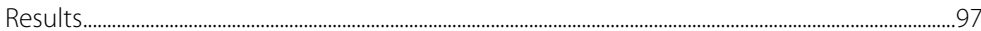

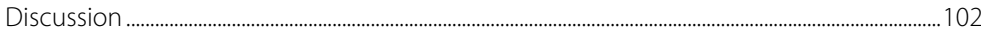

9. Expressed emotion in the caregiver as predictor of behavioural problems in dementia...105

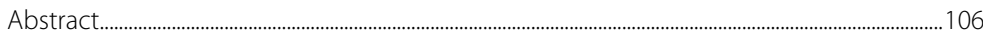

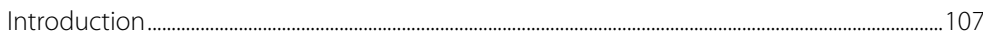

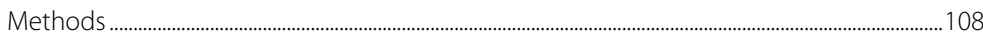

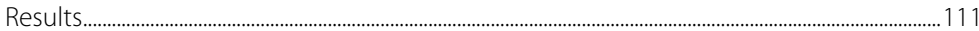

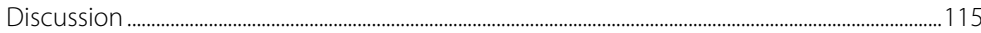

10. Consequences of cognitive functioning in spousal caregivers of dementia patients ..........119

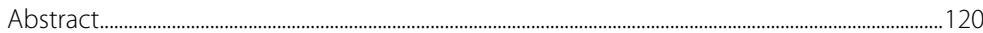

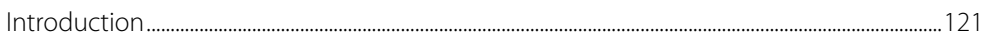

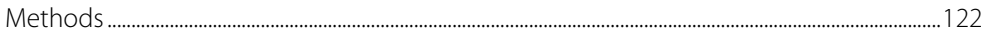

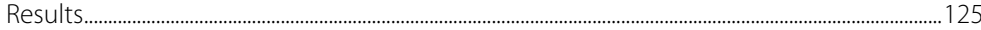

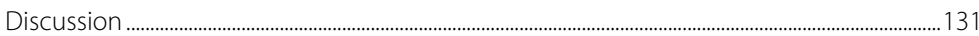

11. Impact of caregivers on behavioural problems in dementia: a qualitative review.................135

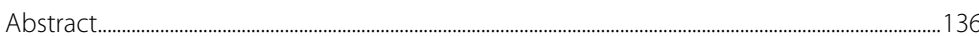

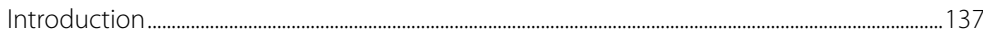

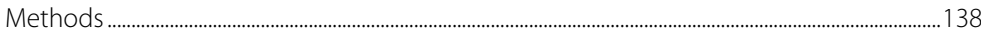

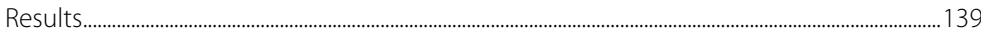

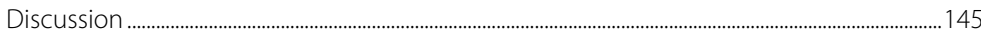

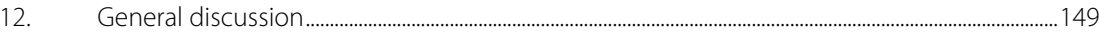

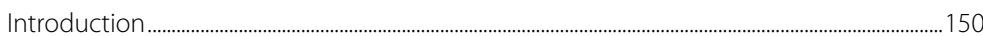

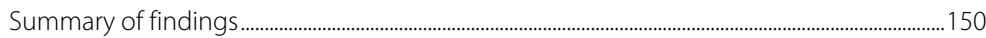

Methodological considerations ............................................................................................................1 153

Conceptual considerations ........................................................................................................................... 157

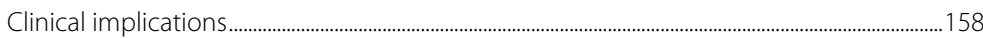

Future directions............................................................................................................................................. 160

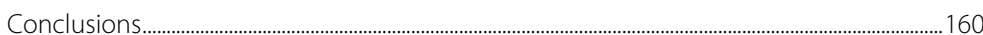

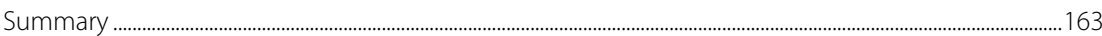

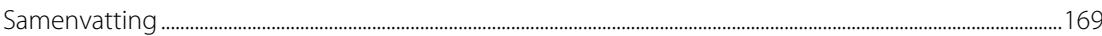

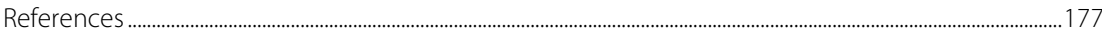

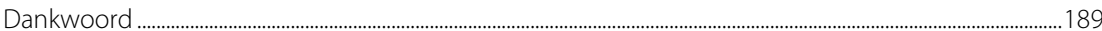

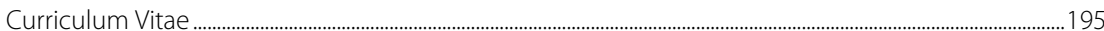




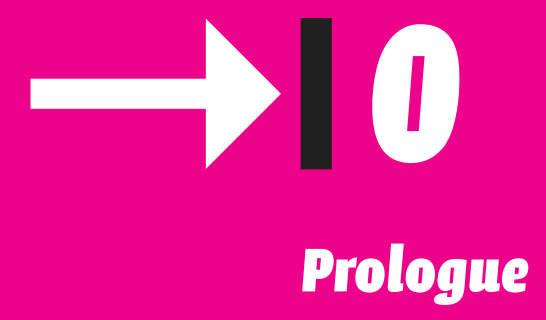




\section{Case descriptions}

Mister A. is 75 years old and referred to a memory clinic by his physician because he is increasingly forgetful. During the interview his wife complains that her husband is very withdrawn. He hardly responds to her questions and sits on the couch all day. They used to go out often together, but now he has no interest at all. She does not understand why he has become so lazy and indifferent.

Mister B. is 68 years old. He takes care of his wife suffering from dementia. She is still doing most of the household chores but with difficulty. For example, she often burns food while cooking dinner or forgets to cook the vegetables. Mister B. now keeps an eye on her in the kitchen and helps her when things go wrong. He does not want to interfere too much with her daily activities and not give her the feeling that he is checking on her.

Mrs. C. is 50 years old and asks help because of problems with her 80 year old mother. She often visits her mother, but she finds these visits increasingly difficult. Her mother is very forgetful and continuously tells stories that are not true. Mrs. C. tries to correct her mother who then reacts irritated or even aggressive. Mrs. C. does not know how to react to her mother in a different way and asks for help.

The patients in these case descriptions were all diagnosed with dementia and cared for by a relative at home. These caregivers mention different cognitive, functional and behavioural problems in the patient and approach these problems in different ways. The question arises what the impact is of dementia related problems on the caregiver and if caregiver functioning can influence the onset and course of these problems. The two-way interaction between patient behavioural problems and caregiver functioning is the main topic of the research described in this book. 


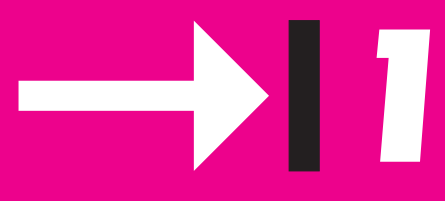

Introduction 


\section{Behavioural problems in dementia}

Alois Alzheimer wrote in 1906 his famous case description of Auguste D., a 55-year old female patient with dementia (Alzheimer 1906). In his brief report he described several psychiatric symptoms, such as delusions, hallucinations and screaming, as important features of her disease. Surprisingly, cognitive symptoms in dementia have traditionally taken a far more prominent place in diagnostic classification systems and clinical research than the psychiatric or behavioural symptoms. Only in the 1980's the interest in behavioural changes in dementia increased and several scales were developed to measure these symptoms (IPA 2003).

The terminology of the behavioural and psychiatric symptoms in dementia has been the subject of debate since. At first, they were described as the non-cognitive symptoms to underline the distinction with the cognitive aspects of dementia. Sometimes they were also referred to as secondary symptoms or complications of dementia. However, these terms tone down the importance of the symptoms, which are now considered to be among the core features of dementia. At a consensus meeting in 1996 of the International Psychiatric Association (IPA) the term Behavioural and Psychological Symptoms of Dementia (BPSD) was suggested, and defined as: symptoms of disturbed perception, thought content, mood or behaviour that frequently occur in patients with dementia (Finkel et al. 1996). This consensus statement provided an umbrella term referring to the wide range of behavioural and psychiatric symptoms that occur in dementia, such as delusions, hallucinations, depression, apathy, aggression, and restlessness.

The term BPSD has shed light on the importance to study and treat these symptoms in dementia. But with the growing understanding of the occurrence, etiology and implications of BPSD, the focus of attention has to expand beyond BPSD to specific symptoms or syndromes. More refinement is needed in research on possible contributing factors, consequences and management of these specific symptoms. This thesis deals with these issues.

In this thesis predictors and consequences of specific behavioural and psychological symptoms or syndromes in dementia are considered, and for practical reasons they will be in general terms referred to as behavioural problems or symptoms. 


\section{Importance of behavioural problems in dementia}

Behavioural symptoms in dementia are of major clinical importance for several reasons. First, they are very common in dementia, with most dementia patients presenting one or more behavioural symptoms during the course of the disease. In a study of Mega et al. (Mega 1996) it was found that as much as $84 \%$ of the dementia patients displayed any behavioural problem. Second, behavioural symptoms are known to cause considerable suffering for both patients and caregivers and decrease their quality of life (Donaldson et al. 1997). Symptoms such as delusions, anxiety and depression are in clinical practice often reported as distressing to patients (Eriksson et al. 2000). Furthermore, behavioural symptoms reduce the functional level of the patient and thus increase disability. Behavioural symptoms are also recognized as major sources of stress in caregivers (Draper et al. 1995). These symptoms are the most difficult problems to manage for family carers, and consequently a common cause of patient institutionalisation (Chan et al. 2003). Behavioural problems are not only affecting family caregivers at home. Also in an institutional setting caring for a patient with behavioural symptoms can be very demanding and distressing for the professional staff. In addition, behavioural problems have a major economic impact, since they increase the risk for institutionalisation, which is the most important source of health care costs in dementia. Finally, as the treatment possibilities of the cognitive symptoms in dementia are limited and often ineffective, the greatest improvement in symptoms can be gained by treating the behavioural problems in the patient.

\section{Behavioural problems and caregiver burden}

The majority of dementia patients are living at home and are usually cared for by family or friends. The terms 'primary caregiver' or 'informal caregiver' have been used to denote someone who voluntary takes on the principal role of taking care for the dementia patient at home. Day-to-day care for a relative with dementia is extremely demanding and has detrimental consequences for the caregiver. Caregivers have high risks for depression and other mental health problems, an increased physical morbidity, higher health-care related costs, and feel more isolated than non-caregivers (Brodaty and Luscombe 1998; Schulz et al. 1995).

Different concepts have been applied in this context to capture the negative aspects of caregiving (Ballard et al. 2001). A distinction has been made between objective and subjective burden. The term objective burden refers to the practical problems associated 
with caregiving such as the patient functional dependence and problem behaviours. Subjective burden refers to the emotional reaction of the caregiver to the demands of the situation, such as feelings of incompetence or exhaustion. Furthermore, the concept of subjective burden can be distinguished from psychiatric symptoms in the caregiver, such as anxiety and depression.

The distinction between objective and subjective burden is important because the association between the severity of impairment in the dementia patient and caregiver well-being is not straightforward. The multi-dimensional changes in the dementia patient have differential effects on the caregiver. It has been shown that the presence of behavioural problems in the dementia patient rather than the cognitive problems or functional dependence appears to be the major contributor to caregiver subjective burden (Coen et al. 1997). Many caregivers do not know how to respond to difficult patient behaviour and, consequently, become frustrated and distressed. These negative emotions may change the caregiver's feelings towards the patient and adversely affect the patient-caregiver relationship. Furthermore, behavioural symptoms have been reported to play an important role in the decision to institutionalise the patient.

The majority of the studies focussing on the association between behavioural problems and caregiver distress have assessed behavioural symptoms in general. Less is known on the differential impact of individual symptoms. Furthermore, caregivers' reaction to problem behaviour is not uniform, some problems may be distressing for some caregivers but not to others. Therefore, studies focusing upon this area have to look not only at behavioural symptoms themselves but also at the reaction of the caregiver to specific behavioural problems.

\section{Behavioural problems and the care environment}

The role of caregivers in the onset and course of patient behavioural problems is less wellresearched than the impact of patient behaviour on caregiver functioning. Behavioural symptoms probably arise from a complex interaction between biological, psychological, and social factors, including caregiver functioning (Lawlor 1996).

Lawton described an ecological model of aging in which a person's behaviour is a function of characteristics of the person and the environment and the interaction between the two (Taft, 1997). According to this model, a person becomes more sensitive 
to the environment when the personal competence decreases, as in dementia patients. The environment includes both the physical and the social care environment, but in dementia research most emphasis has been placed on the physical environment, such as the development of specialized dementia care units. Research into caregiver factors that may cause or exacerbate problem behaviours in the patient is lacking. Still, many caregiver interventions are based upon the assumption that teaching caregivers management skills can reduce patient problem behaviours. The effectiveness of these caregiver interventions is still unclear and needs to be elucidated.

We conclude from this overview that research on the association between behavioural problems in dementia patients and caregiver functioning has gained increased attention, but most studies addressed patient behaviour in general terms and focussed on the unidirectional negative impact of patient behaviour on caregivers. A differential impact of distinct behavioural syndromes may exist and therefore this needs further study. Furthermore, studies on the emergence of patient behavioural problems have mainly focussed on biological and psychological risk factors. Research into the role of social factors, such as caregiver functioning, has been neglected in this area. To examine the complex interaction between patient behaviour and caregiver functioning, we conducted a longitudinal study into the course and risk factors of behavioural problems in dementia, entitled the Maastricht Study of Behaviour in Dementia (MAASBED).

\section{Aims and outline of this thesis}

The following two main questions concerning both sides of the two-way interaction between patient behavioural problems and caregiver functioning are the focus of the present thesis and examined in MAASBED:

1. What are the consequences of behavioural problems in dementia patients for their caregivers?

As noted earlier, behavioural problems rather than cognitive disorders or functional impairment have a negative impact on caregiver functioning. Most studies addressing this issue assessed behavioural problems as a unitary syndrome, but less is known on the differential impact of specific behavioural syndromes. Furthermore, the focus of caregiver studies has to expand beyond Alzheimer's disease to other dementia types such as Frontotemporal dementia, as caregiver experiences may differ between specific diagnostic categories. 
2. Can caregiver functioning influence the onset or course of behavioural problems in the patient?

The underlying cause of behavioural changes in dementia patients is probably an interactive model of genetic, neurobiological, psychological and social factors. Little is known about the role of social aspects, such as caregiver factors, to the etiology of behavioural problems. Caregiver factors related to care management and interpersonal interactions between the patient and caregiver are likely to affect behavioural problems.

Based on these two main questions, several aspects of both sides of the patient-caregiver interaction were examined separately (see figure 1).

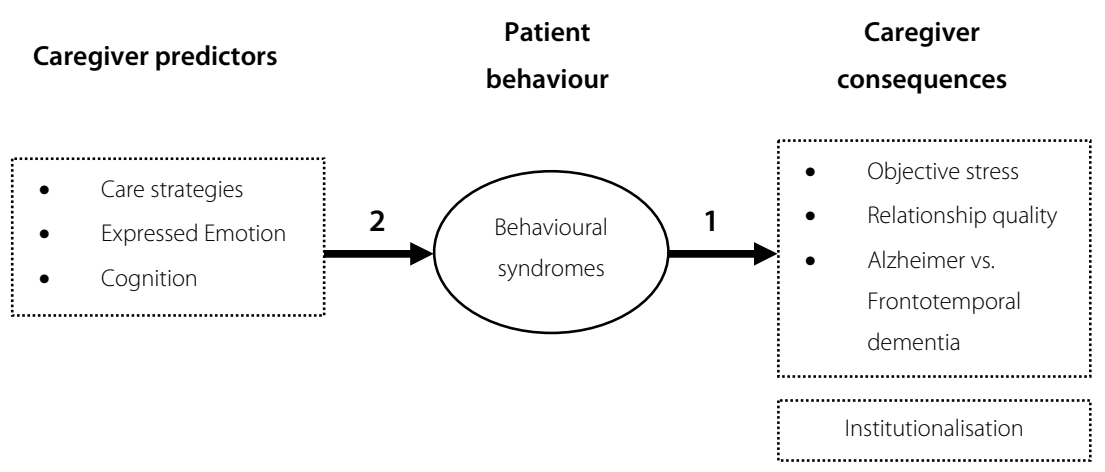

Figure 1. Aspects of the two-way interaction between patient and caregiver as examined in this thesis

The following research questions were posed concerning these aspects of the interaction between patient and caregiver:

1.1 What specific behavioural syndromes can be identified in dementia?

The further refinement of research on behavioural problems in dementia, involves the identification of specific sub-syndromes. In the study described in chapter 3 of this thesis, clusters of symptoms were examined using the 12-item Neuropsychiatric Inventory (NPI) to detect distinct behavioural syndromes. 
1.2 What is the impact of behavioural problems in dementia on caregiver distress?

Caregiver distress can be assessed by subjective as well as objective measures. Little is known about the influence of problem behaviours on the physiological stress response in caregivers. One of the most well-known physiological stress responses is the change in activity of the hypothalamic-pituitary-adrenocortical axis, as reflected in cortisol secretion. The primary goal of the study in chapter 4 was to examine the influence of patient behavioural symptoms on cortisol levels in the caregiver.

In addition, behavioural problems are often considered as a unitary syndrome, but specific symptoms have probably a differential impact on caregivers. Furthermore, as the manifestation of behavioural changes differs between diagnostic groups, there may also be a difference in related caregiver distress. Therefore, the aim of chapter 6 was to examine the impact of individual symptoms in Alzheimer's disease (AD) and Frontotemporal dementia (FTD) on caregiver reported distress, as behavioural problems are a key factor in distinguishing both diagnostic groups.

1.3 What is the differential impact of specific behavioural syndromes in dementia on the quality of the spousal relationship?

Behavioural problems are known to have a negative influence on the relationship between patient and family caregiver. The extent to which behavioural problems are related to negative relationship changes may, however, vary for distinct behavioural syndromes. Identification of this possible differential impact may provide a better understanding of the underlying mechanisms. For this reason, the specific influence of different behavioural syndromes was examined in chapter 5 by means of quantitative as well as qualitative methods.

1.4 Do behavioural problems in dementia influence the decision to institutionalise the patient?

Behavioural symptoms are often reported to be one of the leading risk factors for nursing home admission. However, a review of the literature has shown conflicting results. These contradictory findings may be explained two-fold. First, the differential impact of specific behavioural symptoms has often been neglected. Second, patient institutionalisation may not depend on the presence of behavioural symptoms on itself, but rather on the caregivers' emotional perception of the symptoms. These two hypotheses are tested in chapter 7. 
2.1 Is the interpersonal interaction between patient and caregiver a predictor of patient behavioural problems?

The next question addressed in this thesis is whether a poor interpersonal interaction between patient and caregiver may provoke or exacerbate dysfunctional patient behaviours. An important factor in this regard might be the way caregivers manage their care for the patient. Research into what constitutes a successful strategy is lacking. In chapter 8 distinct care management strategies are identified and examined as possible predictors of patient behavioural problems. Another aspect of interpersonal interaction is the emotional climate between patient and caregiver, as indicated by Expressed Emotion (EE) of the caregiver. EE has already been identified as a risk factor for relapse in schizophrenic and depressed patients. In chapter 9 caregiver EE is studied as a potential risk factor for an adverse course of behavioural problems in dementia.

2.2 What is the impact of caregiver cognitive functioning on patient behavioural problems?

Spousal caregivers are usually of the same age as the dementia patient and therefore at risk for some cognitive decline. Cognitive compromised caregivers may not be able to provide adequate care and to respond accurately to the complex problems they are confronted with every day. However, cognitive status in caregivers has hardly been studied. The aim of the study in chapter 10 is to examine cognitive functioning in spousal caregivers of dementia patients and the consequences for patient behavioural functioning.

\subsection{Do patient behavioural problems decline after caregiver interventions?}

Finally, evidence for the influence of caregiver functioning on patient behavioural problems may be sought in the intervention literature. Many interventions are aimed at learning caregivers skills to manage and reduce patient problem behaviours. A review of caregiver intervention studies is provided in chapter 11. Surprisingly, the majority of the caregiver intervention studies have focussed on caregiver outcomes and relatively few studies assessed the impact of the intervention on patient behavioural problems.

The main findings and implications of the various chapters are discussed in chapter 12. 


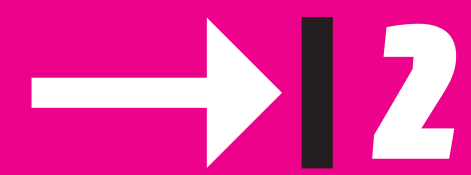

Atms, design and methods 


\section{Introduction}

The importance of the behavioural and psychological symptoms of dementia and the gaps in the literature concerning this issue were stressed in the previous chapter. As stated, we conducted a longitudinal study into the course and risk factors of behavioural problems in dementia, entitled the MAAstricht Study of BEhaviour in Dementia (MAASBED). The study described in this thesis is one of two related projects that stem from MAASBED. In this chapter the MAASBED study is described.

\section{Embedding and aims of the study}

MAASBED is a study into the course and risk factors of behavioural problems in dementia. In this longitudinal project, 199 dementia patients and 119 primary caregivers were psychologically and neuropsychologically examined every six months during the course of two years. The project was a collaboration between the Brain and Behaviour Institute of the Maastricht University, the Memory clinic of the Academic Hospital of Maastricht, and the geriatric division of the Regional Institute for Community Mental Health, Maastricht, the Netherlands. The main aim of MAASBED is to identify risk factors for the behavioural problems in dementia. The study consists of two parts. Part one focuses on the course of the behavioural problems and the impact of patient characteristics on the onset and course of these problems. Several patient factors were examined as possible predictors, such as awareness, pre-morbid personality, neuro-anatomical variables and cognitive functioning. In part two patient behavioural problems were studied in relation to caregiver functioning, which is the focus of this thesis. Several caregiver characteristics were examined as possible predictors of patient behaviour, such as care management strategies, expressed emotion and cognitive functioning. Furthermore, the impact of behavioural problems on caregiver subjective and objective (cortisol) stress and relationship quality was assessed.

\section{Sample}

\section{Characteristics}

Patients were consecutively referred by the Memory Clinic of the Academic Hospital Maastricht or the geriatric division of the Regional Institute for Community Mental Health (RIAGG). All patients were diagnosed with dementia and living at home at baseline. Data on clinical symptoms, neuropsychological testing, laboratory studies and neuro-imaging were discussed in plenary clinical rounds to diagnose patients with dementia according 
to DSM-IV criteria (American Psychiatric Association 1994). Diagnoses of Alzheimer's disease, vascular dementia, and dementia with Lewy bodies (DLB) were made in accordance with NINCDS/ADRDA (McKhann et al. 1984), NINDS/AIREN (Roman et al. 1993), and DLB Consensus criteria (McKeith et al. 1996) respectively. Of the 199 participating patients there were 146 patients with Alzheimer's Disease (AD), 32 vascular dementia, 3 Frontotemporal dementia, 5 Parkinson's dementia, 2 Lewy Body dementia, 4 primary progressive aphasia, 1 alcohol dementia and 6 mixed dementia (AD/vascular). There were 119 informal caregivers participating in part two of the study. Caregivers were included if they were the primary caregiver and had contact with the patient at least once a week. Patient and caregiver characteristics are shown in table 1.

\section{Table 1. Baseline characteristics of patients and caregivers}

\begin{tabular}{|c|c|c|}
\hline & $\begin{array}{l}\text { Patient } \\
(n=199)\end{array}$ & $\begin{array}{l}\text { Caregiver } \\
(n=119)\end{array}$ \\
\hline & Mean (SD) & Mean (SD) \\
\hline Age & $76.4(7.9)$ & $63.8(12.2)$ \\
\hline Sex ${ }^{1} \mathrm{~m} / \mathrm{f}(\%$ male $)$ & $85 / 114(43 \%)$ & $42 / 77(35 \%)$ \\
\hline Illness duration (months) & $36.2(25.9)$ & \\
\hline \multicolumn{3}{|l|}{ 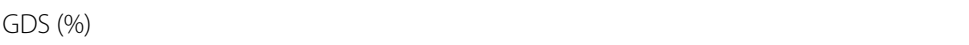 } \\
\hline Moderate 3-4 & 71.4 & \\
\hline Severe 5-6 & 28.6 & \\
\hline \multicolumn{3}{|l|}{ Relationship (\%) } \\
\hline Spouse & & $64(54 \%)$ \\
\hline Child & & $47(40 \%)$ \\
\hline Other & & $8(7 \%)$ \\
\hline Care duration (months) & & $27.9(25.5)$ \\
\hline Contact intensity (hours/week) & & $92.2(71.3)$ \\
\hline MMSE score & $18.1(4.7)$ & \\
\hline CAMCOG score & $58.5(15.6)$ & \\
\hline
\end{tabular}

GDS = Global Deterioration Scale | MMSE = Mini-Mental State examination | CAMCOG = Part B of CAMDEX-R-N 1. values represent number of males and females

\section{Refusal}

Of the 199 dementia patients included in the study at baseline, 161 (80\%) had a primary caregiver. Hundred-nineteen (73.9\%) primary caregivers agreed to participate in part two of the study and 42 (26.1\%) refused. Reasons for refusal were: no time (10; $23.8 \%$ ), too distressing (14; $33.3 \%)$, no gain $(8 ; 19 \%)$, and 10 (23.8\%) caregivers gave no reason. 
Table 2. Baseline differences between patients and caregivers of the Memory Clinic ( $n=$ 61) and the RIAGG ( $n=58)$

\begin{tabular}{|c|c|c|c|c|}
\hline & $\begin{array}{l}\text { Memory } \\
\text { Clinic } \\
n=61\end{array}$ & $\begin{array}{l}\text { RIAGG } \\
n=58\end{array}$ & $\begin{array}{l}\text { Test } \\
\text { value }\end{array}$ & $P$ value \\
\hline & Mean (SD) & Mean (SD) & & \\
\hline \multicolumn{5}{|l|}{ Patient } \\
\hline Age & $74.7(8.5)$ & $82.4(6.1)$ & $t=5.6$ & $<0.001$ \\
\hline Sex m/fำ (\% male) & $27 / 34(44.3 \%)$ & 22/36 (37.9\%) & $\chi 2=0.9$ & 0.343 \\
\hline Illness duration (months) & $47.5(31.7)$ & $38.3(29.9)$ & $t=-1.6$ & 0.109 \\
\hline \multicolumn{5}{|l|}{ GDS (\%) } \\
\hline Moderate 3-4 & $45(73.7 \%)$ & $41(70.7 \%)$ & $\chi 2=0.1$ & 0.707 \\
\hline Severe 5-6 & $16(26.3 \%)$ & $17(29.3 \%)$ & & \\
\hline MMSE & $18.3(5.1)$ & $18.3(4.3)$ & $t=0.05$ & 0.954 \\
\hline \multicolumn{5}{|l|}{ IDDD } \\
\hline Initiative & $23.4(10.3)$ & $21.5(8.8)$ & $t=-1.5$ & 0.296 \\
\hline Performance & $17.5(11.1)$ & $22.8(10.1)$ & $t=2.7$ & 0.008 \\
\hline NPI-score & $19.0(18.5)$ & $24.7(24.9)$ & $t=1.5$ & 0.144 \\
\hline \multicolumn{5}{|l|}{ Caregiver } \\
\hline Age & $63.3(11.9)$ & $64.4(12.4)$ & $t=-0.5$ & 0.613 \\
\hline Sex m/f' (\% male) & 24/37 (39.3\%) & $18 / 40$ (31\%) & $\chi 2=0.9$ & 0.343 \\
\hline Education $^{2}$ & $3.8(1.9)$ & $3.2(1.8)$ & $\mathrm{t}=-1.8$ & 0.068 \\
\hline \multicolumn{5}{|l|}{ Relationship (\%) } \\
\hline Spouse & $43(70.5 \%)$ & $21(36.2 \%)$ & & \\
\hline Child & $15(24.6 \%)$ & $32(55.2 \%)$ & $\chi 2=14.1$ & 0.001 \\
\hline Other & $3(4.9 \%)$ & $5(8.6 \%)$ & & \\
\hline Care duration (months) & $29.1(24.6)$ & $26.9(26.6)$ & $t=-0.5$ & 0.650 \\
\hline Contact intensity (hrs/week) & $117.1(62.0)$ & $72.4(71.6)$ & $\chi 2=11.6$ & 0.001 \\
\hline NPI-distress & $10.0(8.2)$ & $12.2(11.4)$ & $t=1.5$ & 0.144 \\
\hline MADRS & $7.1(5.5)$ & $9.6(6.7)$ & $t=2.2$ & 0.028 \\
\hline SOC & $25.0(5.6)$ & $22.4(6.0)$ & $t=-2.5$ & 0.014 \\
\hline
\end{tabular}

GDS $=$ Global Deterioration Scale $\mid$ MMSE $=$ Mini-Mental State examination $\mid$ IDDD = Interview of Daily living activities in Dementia $\mid \mathrm{NPI}=$ NeuroPsychiatric Inventory | MADRS = Montgomery Asberg Depression Rating Scale $\mathrm{SOC}=$ Sense of Competence

1. values represent number of males and females | 2. ranging from (1) primary school to (8) university degree

\section{Memory clinic versus RIAGG}

Hundred-and-nineteen caregivers and patients were included in the second part of MAASBED of which 61 (51.3\%) patients were referred by the Maastricht Memory Clinic and 58 (48.7\%) patients by the RIAGG. Using patients from both sites may have caused a referral bias, because a difference exists in reasons for referral between both populations. Patients with behavioural problems are probably more often referred to the RIAGG, while patients of the memory clinic are often referred for early diagnostics. Examination of differences between both groups showed that patients from the RIAGG group were older 
and performed less ADL activities; their caregivers were more often children, had less contact hours with the patient, and reported more depressive symptoms and lower feelings of competence compared to the memory clinic group (table 2).

Table 3. Measures assessed per visit to patient and caregiver

\begin{tabular}{|c|c|c|c|c|c|c|c|}
\hline & \multirow[t]{2}{*}{ Informant } & \multirow[t]{2}{*}{ Screening } & \multicolumn{5}{|c|}{ Months } \\
\hline & & & 0 & 6 & 12 & 18 & 24 \\
\hline \multicolumn{8}{|l|}{ Patient measures } \\
\hline Informed Consent & $\begin{array}{l}\mathrm{P} \text { (atient) / } \\
\mathrm{C} \text { (aregiver) }\end{array}$ & $\bullet$ & & & & & \\
\hline In/exclusion criteria & $\mathrm{P} / \mathrm{C}$ & $\bullet$ & & & & & \\
\hline DSM-IV & & $\bullet$ & & & & & \\
\hline Psychiatric (hetero)anamnesis & $\mathrm{P}$ & & $\bullet$ & & & & \\
\hline Medical (hetero)anamnesis & $\mathrm{P} / \mathrm{C}$ & & $\bullet$ & & & & \\
\hline Demographic variables & $\mathrm{P} / \mathrm{C}$ & & $\bullet$ & & & & \\
\hline NPI & C & & $\bullet$ & $\bullet$ & $\bullet$ & $\bullet$ & $\bullet$ \\
\hline MMSE & $P$ & & $\bullet$ & $\bullet$ & $\bullet$ & $\bullet$ & $\bullet$ \\
\hline IDDD & $C$ & & $\bullet$ & & $\bullet$ & & $\bullet$ \\
\hline GDS & $\mathrm{P} / \mathrm{C}$ & & $\bullet$ & & $\bullet$ & & $\bullet$ \\
\hline Treatment information & C & & $\bullet$ & $\bullet$ & $\bullet$ & $\bullet$ & $\bullet$ \\
\hline \multicolumn{8}{|l|}{ Caregiver measures } \\
\hline In/exclusion criteria & C & $\bullet$ & & & & & \\
\hline Informed Consent & C & $\bullet$ & & & & & \\
\hline Demographic variables & C & & $\bullet$ & & & & \\
\hline Neuropsychological tests & C & & $\bullet$ & & & & \\
\hline NPI-Distress & C & & $\bullet$ & $\bullet$ & $\bullet$ & $\bullet$ & $\bullet$ \\
\hline SCL-90 & C & & $\bullet$ & $\bullet$ & $\bullet$ & $\bullet$ & $\bullet$ \\
\hline MADRS & C & & $\bullet$ & & $\bullet$ & & $\bullet$ \\
\hline RAND 36 & C & & $\bullet$ & & $\bullet$ & & $\bullet$ \\
\hline NEO-FFI & C & & $\bullet$ & & & & \\
\hline UCL & C & & $\bullet$ & & & & \\
\hline Mastery & C & & $\bullet$ & & & & \\
\hline FMSS & C & & $\bullet$ & & $\bullet$ & & $\bullet$ \\
\hline CAS & C & & $\bullet$ & $\bullet$ & $\bullet$ & $\bullet$ & $\bullet$ \\
\hline SOC & C & & $\bullet$ & $\bullet$ & $\bullet$ & $\bullet$ & $\bullet$ \\
\hline SSL12-I & C & & $\bullet$ & & $\bullet$ & & $\bullet$ \\
\hline Social network & C & & $\bullet$ & & $\bullet$ & & $\bullet$ \\
\hline Sleep quality & C & & $\bullet$ & $\bullet$ & $\bullet$ & $\bullet$ & $\bullet$ \\
\hline Cortisol & C & & $\bullet$ & & $\bullet$ & & $\bullet$ \\
\hline PSS & C & & & & & & \\
\hline Qualitative interview & C & & $\bullet$ & & $\bullet$ & & $\bullet$ \\
\hline
\end{tabular}

$\mathrm{NPI}=$ Neuropsychiatric Inventory $\mid$ MMSE $=$ Mini Mental State Examination $\mid$ IDDD = Interview of Daily living activities in Dementia $\mid$ GDS = Global Deterioration Scale $\mid$ SCL-90 = Symptom Checklist-90 | MADRS = Montgomery Asberg Rating Scale | NEO-FF-I = Neuroticism-Extroversion-Openness Five Factor personality Inventory $\mid \mathrm{UCL}=$ Utrecht Copinglist $\mid$ FMSS = Five Minute Speech Sample $\mid$ CAS = Caregiver Activities Scale $\mid$ SOC = Sense of Competence Questionnaire | SSL12-I = Social Support List | PSS = Perceived Stress Scale 


\section{Assessment}

\section{Procedure}

Informed consent was obtained from all subjects. Patient and caregiver assessment were conducted at baseline, 6-, 12-, 18-, and 24 months follow-up by independent, clinically experienced, trained psychologists. Assessments at baseline, 12-, and 24 months were more extensive than at 6-, and 18 months. Patient assessment consisted of a neuropsychological examination; caregiver assessment consisted of a structured and semi-structured interview, and questionnaires. In addition a neuropsychological examination was performed in a sub-sample of only spousal caregivers, because spouses were usually of the same age as the patient and therefore at risk for some age-related cognitive decline. Furthermore, later on in the study it was decided to assess salivary cortisol as a measure of objective stress, thus these data were also collected in only a subsample of the caregivers. Main outcome measures were the Neuropsychiatric Inventory (NPI) to assess behavioural symptoms in the patient and caregiver distress, and the Short Sense of Competence Questionnaire (SOC) to assess caregiver feelings of competence to care for the patient. These instruments were administered at all visits. All measures assessed per visit are shown in table 3. Not all measures were used in the studies described in this thesis. The most important measures are discussed in the next paragraph.

\section{Measures}

- NeuroPsychiatric Inventory

The NPI was used to assess patient's behavioural and psychological problems (Cummings 1997). Several rating scales have been developed for this purpose; however the NPI is widely used and assesses a wide range of behaviours. In addition, the NPI distinguishes symptom frequency and severity, and facilitates rapid behavioural assessment through the use of screening questions. The NPI is based on a structured interview with a caregiver who is familiar with the patient's daily functioning. After explaining the purpose of the interview, a screening question is asked for each neuropsychiatric domain, followed by sub-questions if the response to the screening question suggests the presence of abnormalities in that domain. The behavioural domains assessed with the NPI are: delusions, hallucinations, dysphoria, anxiety, agitation, euphoria, apathy, irritability, disinhibition, aberrant motor behaviour, night-time disturbances and changes in eating 
behaviours. For each domain, the caregiver is asked to rate the frequency on a scale from 1 (occasionally) to 4 (very frequently), and the severity on a scale from 1 (mild) to 3 (severe). A total score is obtained by multiplying the frequency and severity score for each domain (maximum score per domain is 12), and adding them up (maximum total score is 144). In addition, for each domain a caregiver distress score is rated on a six-point scale from 0 (no distress) to 5 (extreme distress). Recently, several psychometric aspects of the Dutch translation of the NPI were published (Kat et al. 2002).

- Short Sense of Competence Questionnaire

The SOC is a shortened form of the 27-item Sense of Competence Questionnaire (Vernooij-Dassen et al. 1999) and assesses caregivers' feelings of being capable of caring for a demented person. The advantage of the SOC is that it refers to the specific situation of caregiving and does not measure a general characteristic of the caregiver. In addition, the concept of competence is preferred because it refers to caregiver capabilities, while caregiver burden is more related to the patient (Vernooij-Dassen et al. 1996). The SOC is based on the family-crisis model and includes issues from the Zarit's Burden Scale. Three domains are distinguished: (1) satisfaction with the demented person as a care recipient; (2) satisfaction with one's own performance as a caregiver; and (3) consequences of involvement in care for the personal life of the caregiver. The SOC consists of 7 items rated on a 5 -point scale from 1 (agree very strongly) to 5 (disagree very strongly). The validity and reliability of the SOC were assessed in a study of 141 caregivers of community-living dementia patients (Vernooij-Dassen et al. 1999).

- Montgomery Asberg Rating Scale

The MADRS (Montgomery and Asberg 1979), a structured interview, was used to assess depressive symptoms in the caregiver. As the MADRS contains relatively few somatic items, it is particularly suitable for the wide range in our sample, including elderly caregivers. Ratings from 0 to 6 on 10 items were summed.

- $\quad$ Five Minute Speech Sample

Level of Expressed Emotion was assessed by the FMSS (Magana et al. 1986). This instrument is a less time consuming alternative to the Camberwell Family Interview (CFI) to measure Expressed Emotion. In the FMSS, a caregiver is asked to speak freely for $5 \mathrm{~min}$ about the patient and how they get along together. The speech sample is audiotaped and transcribed verbatim. A qualified rater then codes the sample for 4 dimensions: (1) the 
initial statement, (2) quality of the relationship, (3) criticism, and (4) emotional overinvolvement (EOI). Caregivers are classified as high EE if they scored on the critical scale and/or the EOI scale, otherwise they are classified as low EE.

- $\quad$ Qualitative interviews

Qualitative interviews were used in addition to quantitative methods to study the experiences of the caregiver, relational changes between patient and caregiver, and the context of patient problem behaviours. Qualitative methods are particularly appropriate to describe experiences of illness in the context of everyday life, issues of process, and underlying factors of associations (Britten 1995). Since caregiver experiences and the social context of patient behaviour are the main issues of this study, qualitative methods are considered to be useful in addition to quantitative methods. A semi-structured interview was used with questions addressing the way caregivers manage the caregiving situation and how they deal with dementia-related problems. The interviews were taperecorded and then transcribed verbatim for analysis.

- Neuropsychological examination

Cognitive functioning in spousal caregivers was assessed by means of neuropsychological tasks measuring verbal memory (Auditory Verbal Learning Test; Brand and Jolles 1985), speed of information processing (Letter Digit Coding Test; Smith 1968), cognitive flexibility (Stroop; Houx et al. 2002; Stroop 1935), global cognitive functioning (MMSE; Folstein et al. 1975), and general intelligence (shortened GIT; Luteijn and van der Ploeg 1983).

- Cortisol

Cortisol secretion was assessed to measure the physiological stress response in caregivers. Cortisol was assessed from saliva collected with a cotton dental roll, stored in a capped plastic vial (Salivette; Sarstedt, Etten-Leur, the Netherlands). Saliva collection is noninvasive and interferes only minimally with normal daily routines, in contrast to blood sampling. Salivary cortisol represents the unbound ("free"), biologically active fraction of total cortisol; it correlates highly with free plasma cortisol (Kirschbaum and Hellhamer 1994). 


\section{Loss to follow-up}

Of the 119 primary caregivers included in the study at baseline, there were 97 (81.5\%) caregivers still participating at 1 year follow-up and 75 (63\%) caregivers at 2 year followup. Missing values in the follow-up were due to refusal $(18 ; 15.1 \%)$ or death of the patient (26; 21.8\%). Caregivers lost to follow-up were significantly older ( $t=-2.8, p=0.006$ ). Furthermore, dementia in the patients was more severe, as measured with the Global Deterioration Scale $(t=-2.0, p=0.045)$, and patients were more cognitively impaired on the MMSE ( $t=2.1, p=0.034)$. There were no other differences in patient or caregiver characteristics.

\section{Practical considerations}

Patient and caregiver assessments were mostly conducted simultaneously in the home of the participants by two independent raters. The extensive assessments took approximately 2 hours, while the duration of the other assessments was around 1 hour. Caregivers were asked to fill in a booklet with questionnaires during the next week. Caregiver and patient assessments were performed by two teams of two psychologists. They received training in test administration from a highly experienced test assistant. During the course of the study problems and other issues were discussed. Psychologists attended the weekly clinical rounds, to ensure enrolment of new patients in the study. Feedback of the individual test results was given to the clinician concerned by means of a standardized report. Study participants received a Christmas card every year in appreciation of their participation.

\section{Data-management}

Data from structured interviews, questionnaires and neuropsychological assessments were entered into a FileMaker Pro 5 database. Data integrity was ensured by matching double entered data. The database was password protected. Data was converted to SPSS 10.0 for statistical analyses. 
Chapter 2. Aims, design and methods 


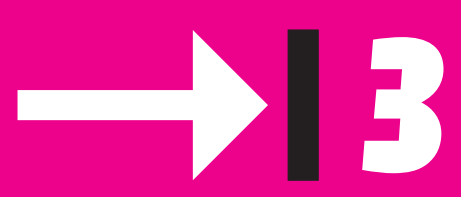

\section{Factor analysis of the Neuropsychiatric Inventory (INPI)}


Chapter 3. Factor analysis of the Neuropsychiatric Inventory (NPI)

\section{Abstract}

Objectives: Behavioural problems in dementia can be diagnosed at both a symptomatic and syndromal level. Several rating scales have been designed to assess behavioural symptoms, of which the Neuropsychiatric Inventory (NPI) is often used. The aim of this study was to detect behavioural sub syndromes of the 12-item NPI.

Methods: Cross-sectional data of 199 patients with dementia living in the community were collected. Behavioural problems were measured with the NPI. Principal component analysis (with Varimax-rotation) was used for factor analysis.

Results: Results showed the presence of three behavioural sub syndromes: mood/apathy, psychosis, and hyperactivity. Anxiety was regarded as a separate symptom. The sub syndrome mood/apathy was the most common, occurring in almost $80 \%$ of the patients, versus psychosis and hyperactivity, which occurred in $37 \%$ and $60 \%$ of the patients, respectively.

Conclusions: Our study provides evidence for the existence of behavioural sub syndromes in dementia. The three sub syndromes can give insight into possible relationships between behavioural symptoms, their underlying cause and risk factors, and clinical correlates. 


\section{Introduction}

Non-cognitive, or behavioural and psychological symptoms in dementia, often abbreviated as BPSD (Finkel 1998), are associated with more caregiver distress and increase the likelihood of institutionalization (Benoit 1999; Cummings 1994; De Deyn 1998; Finkel 1998; Kaufer 1998; Mega 1996; Tariot et al. 1995). Such problems are diagnosed at both a symptomatic and syndromal level. Several rating scales have been designed to assess behavioural and psychological symptoms, of which the Neuropsychiatric Inventory (NPI) is most often used to date (Cummings 1994).

It is important to study behavioural sub syndromes in dementia instead of investigating separate behavioural symptoms because these sub syndromes may point to a common neurobiological pathogenesis, or may react to the same treatment (Lawlor and Ni Bhriain 2001). For example pharmacological studies (Kaufer et al. 1998; Lawlor and Ni Bhriain 2001; Street et al. 2000) have shown that treatments have a consistent effect on behavioural problems in dementia when studying behavioural sub syndromes, but not when studying individual symptoms. Data reduction, through the reduction of a number of symptoms into sub syndromes, leads to greater possibility of finding associations.

In the DSM-IV (American Psychiatric Association 1994), dementia is sub typed in terms of the sub syndromes of uncomplicated dementia, or dementia with depressed mood, delusions, or delirium. The aggregation of symptoms into clusters as proposed by the DSM-IV stems from the traditional nosological classification of general psychiatry, and therefore does not necessarily apply directly to patients with dementia. It needs to be determined whether behavioural sub syndromes, i.e. clustering of psychopathological symptoms, occur in populations of patients with representative dementia.

Studies involving factor analysis, have been published recently (Devanand 1992b; Frisoni et al. 1999; Fuh et al. 2001; Hope 1997; Kaufer et al. 1998; McShane 2000). These earlier studies made use of a variety of assessment instruments to diagnose BPSD, including an earlier shorter version of the NPI (Frisoni et al. 1999). The aim of this study was to identify behavioural sub syndromes of the current version of the Neuropsychiatric Inventory (NPI) in consecutive patients with dementia referred to a mental outpatient service for the elderly. 


\section{Methods}

\section{Patients}

Subjects were 199 ambulatory patients with dementia according to DSM-IV (American Psychiatric Association 1994). Standardized clinical diagnostic criteria were used to characterize the type of dementia. Diagnoses of Alzheimer's disease, vascular dementia, and dementia with Lewy bodies (DLB) were made in accordance with NINCDS/ADRDA (McKhann et al. 1984), NINDS/AIREN (Roman et al. 1993), and DLB Consensus criteria (McKeith et al. 1996) respectively.

The patients were included in the Maastricht Study of Behaviour in Dementia (abbreviated as MAASBED). MAASBED focuses on the course and risk factors of behavioural problems in dementia. The project, funded by the Dutch National Research Council (NWO), is a 2-year prospective follow-up study of 199 patients with dementia and 119 informal caregivers. Patients and their caregivers are seen at 6- month intervals. Patients were enrolled from the Maastricht Memory Clinic of the University Hospital Maastricht, and the Department of Mental Health for the Elderly of the regional Institute of Ambulatory Mental Health of Maastricht. Both are psychiatric based clinics. Most of the patients were referred by a local general practitioner because of cognitive deficits, while in some cases the presence of behavioural problems was the reason for referral. The included patients were a representative sample of dementia patients living in the community. Because the presence of behavioural syndromes in a consecutive demented population in need of care was our main point of interest, all dementia patients, regardless of etiology, were included.

Patients were excluded if they lived in a nursing home at the beginning of the study and if there was no caregiver who had contact with the patient at least once a week. Written consent was given by the caregiver, and when possible also by the patient. This study was approved by the Medical Ethics Committee of the University Hospital Maastricht.

The cross-sectional data from the baseline data of MAASBED were used for this study.

\section{Neuropsychiatric Inventory}

The NPI (Cummings 1994) is a short, reliable, informant-based rating scale developed to assess psychopathology in dementia patients. The original NPI assesses ten behavioural symptoms. The current 12-item version (Cummings 1997) evaluates 12 Neuropsychiatric symptoms which are commonly observed in dementia: delusions, hallucinations, agitation / aggression, dysphoria / depression, anxiety, apathy, irritability, euphoria, 
disinhibition, aberrant motor behaviour, night- time behaviour disturbances, and appetite and eating abnormalities. The two latter mentioned symptoms are added in the current version. The severity and frequency of each neuropsychiatric symptom were scored on the basis of structured questions administered to the patient's caregiver. The score for each symptom was obtained by multiplying severity (1-3) with frequency (1-4). The summed symptom scores give the total NPI score. The NPI also assesses the amount of caregiver distress related to each neuropsychiatric symptom. The content validity, concurrent validity, inter-rater reliability, and test-retest reliability of the NPI have been established earlier (Cummings and McPherson 2001). The NPI may help to distinguish between different causes of dementia and is sensitive to treatment effects (Cummings 1997).

\section{Procedure}

Trained psychologists administered cognitive tests to the patients and additional information was gathered from the primary caregivers.

Cognitive tests administered to the patients included the Mini-Mental State Examination (Folstein et al. 1975) and part B of the Cambridge Examination for Mental Disorders of the Elderly (Derix et al. 1991). The severity of dementia was rated with the Global Deterioration Scale (GDS)(Reisberg et al. 1982). The Cornell Scale for Depression in Dementia (CSDD) (Alexopoulos 1988) was administered to the caregivers.

\section{Data analysis}

Statistical analysis was performed with the Statistical Package for Social Sciences (SPSS), version 10. To detect behavioural sub syndromes, we performed a principal component analysis on the NPI items, using an orthogonal rotational procedure (Varimax). Factors were selected on the basis of eigenvalues greater than 1. Factor loadings equal or greater than 0.45 were included.

At first, the analysis was performed on the total dementia population because behaviour problems were the starting point of the study, regardless of etiology.

Secondly, in order to assess possible differences between patient subgroups, three separate analyses were performed for mild (Global Deterioration Scale (GDS) 3-4) and severe (GDS 5-6) dementia stages and for Alzheimer patients only. Cronbach's alpha coefficients were used to assess the internal consistency of the factors. Spearman's rank correlations were calculated between the factors and the Cornell Scale for Depression in 
Dementia, to determine the correlation between the factor including depression and an external validation measure.

\section{Results}

\section{Characteristics of the subject group}

Of the 199 subjects, 89 were recruited through the Maastricht Memory Clinic and 110 through the Department of Mental Health of the Elderly, from the regional Institute of Ambulatory Mental Health of Maastricht.

Of the 199 referrals, 146 met the DSM-IV criteria for dementia of the Alzheimer's type. Thirty-two patients had vascular dementia, 6 patients with mixed Alzheimer's disease and vascular etiology, 3 frontal dementia, 5 Parkinson dementia, 2 Lewy body dementia, 4 primary progressive aphasia, and 1 alcohol dementia. Most patients had a mild form of dementia (71.4\%). Patient characteristics are described in table 1.

Table 1. Baseline characteristics of patients $(n=199)$ and caregivers $(n=119)$

\begin{tabular}{|c|c|c|}
\hline & $\begin{array}{l}\text { Patient } \\
(n=199)\end{array}$ & $\begin{array}{l}\text { Caregiver } \\
(\mathrm{n}=119)\end{array}$ \\
\hline & Mean (SD) & Mean (SD) \\
\hline Age & $76.4(7.9)$ & $63.8(12.2)$ \\
\hline Sex m/f' & $85 / 114$ & $42 / 77$ \\
\hline Illness duration (months) & $36.2(25.9)$ & \\
\hline \multicolumn{3}{|l|}{ GDS (\%) } \\
\hline Moderate 3-4 & 71.4 & \\
\hline Severe 5-6 & 28.6 & \\
\hline \multicolumn{3}{|l|}{ Relationship (\%) } \\
\hline Spouse & & $64(54 \%)$ \\
\hline Child & & $47(40 \%)$ \\
\hline Other & & $8(7 \%)$ \\
\hline Care duration (months) & & $27.9(25.5)$ \\
\hline Contact intensity (hours/week) & & $92.2(71.3)$ \\
\hline MMSE score & $18.1(4.7)$ & \\
\hline CAMCOG score & $58.5(15.6)$ & \\
\hline
\end{tabular}

GDS - Global Deterioration Scale | MMSE - Mini-Mental State examination | CAMCOG - Part B of CAMDEX-R-N

1. values represent number of males and females 
Apathy and depression were the most common symptoms, each being present in about $60 \%$ of the patients (table 2). The rarest symptoms were euphoria (7\%), disinhibition (13\%), and hallucinations (13\%). Eighteen patients did not have any NPI symptoms.

Table 2. Mean NPI scores (severity * frequency; range 0-12) and \% patients with symptoms; $n=199$

\begin{tabular}{lll}
\hline NPI items & Mean \pm SD & $\begin{array}{l}\text { \% Patients with symptom } \\
\text { (any severity) }\end{array}$ \\
\hline Apathy & $3.27 \pm 3.72$ & 59.3 \\
Depression & $3.48 \pm 4.22$ & 57.3 \\
Irritability & $2.37 \pm 3.83$ & 39.7 \\
Anxiety & $1.99 \pm 3.53$ & 39.2 \\
Delusions & $1.95 \pm 3.41$ & 34.7 \\
Aberrant motor behaviour & $2.23 \pm 3.73$ & 34.7 \\
Agitation & $1.50 \pm 2.94$ & 28.6 \\
Appetite and eating abnormalities & $1.73 \pm 3.46$ & 24.6 \\
Night-time behaviour disturbances & $1.22 \pm 2.98$ & 18.1 \\
Hallucinations & $0.83 \pm 2.52$ & 13.1 \\
Disinhibition & $0.61 \pm 2.12$ & 12.6 \\
Euphoria & $0.34 \pm 1.54$ & 7.0 \\
\hline
\end{tabular}

\section{Factor analyses}

The results of the exploratory factor analyses of the total patient group are shown in table 3. Principal component analysis, using the criterion of eigenvalues greater than 1 , reduced the 12 variables to three factors. The three factors explained $55.1 \%$ of the variance in the data. The first factor (29.5\% of the total variance) denoted a dimension representing 'hyperactivity', and had high loadings on agitation, euphoria, irritability, disinhibition, and aberrant motor behaviour. The second factor (14.5\% of the total variance) represented a 'mood/apathy' dimension and had high loadings on depression, apathy, night-time behaviour disturbances, and appetite and eating abnormalities. The third factor $(11.1 \%$ of the total variance) represented a 'psychosis' dimension and had high loadings on delusions, hallucinations, and anxiety. However, comparable factor loadings on different factors were found for the symptoms anxiety, aberrant motor behaviour, and night- time behaviour disturbances. The loading of anxiety on the psychosis factor was 0.50 and on the mood factor 0.42 . In addition, a reliability analysis revealed that if anxiety was omitted from the factor psychosis, the Cronbach's alpha coefficient increased substantially (from 0.64 to 0.72 ). Therefore, anxiety was excluded from the factor psychosis. Cronbach's alpha 
did not show improvements if motor behaviour or night-time behaviour disturbances were removed from the factors hyperactivity (Cronbach's alpha: 0.73) and mood/apathy (Cronbach's alpha: 0.63 ) respectively.

Separate analyses were performed for patients with mild (GDS 3-4) or severe (GDS 5-6) dementia. They resulted in only slightly different factor structures. In mild dementia, aberrant motor behaviour was not included in factor 1 'hyperactivity' but in factor 2 'mood/apathy', whereas 'night-time behaviour disturbances' was not included in factor 2 but in factor 3 'psychosis'. In severe dementia, aberrant motor behaviour had the highest correlation with the factor 3 'psychosis', and anxiety was not included in factor 3 but in factor 2 'mood/apathy'.

The analysis for patients with Alzheimer's disease $(n=146)$ revealed a factor structure analogous to that found for the total patient group, and in particular resembled that of the mild dementia stages. Reliable sub analysis of the non-Alzheimer patient groups could not be done because of the small sample sizes.

Table 3. Factor analysis of the NPI variables in the total group

\begin{tabular}{llll}
\hline & $\begin{array}{l}\text { Factor 1: } \\
\text { Hyperactivity }\end{array}$ & $\begin{array}{l}\text { Factor 2: } \\
\text { Mood/Apathy }\end{array}$ & $\begin{array}{l}\text { Factor 3: } \\
\text { Psychosis }\end{array}$ \\
\hline & & & \\
Delusions & 0.222 & 0.021 & $\mathbf{0 . 8 3 0}$ \\
Hallucinations & 0.055 & 0.002 & $\mathbf{0 . 8 4 3}$ \\
Agitation & $\mathbf{0 . 7 2 1}$ & 0.312 & -0.086 \\
Depression & 0.169 & $\mathbf{0 . 6 3 4}$ & 0.178 \\
Anxiety & -0.121 & 0.423 & $\mathbf{0 . 5 0 0}$ \\
Euphoria & $\mathbf{0 . 7 1 6}$ & -0.095 & 0.066 \\
Apathy & 0.118 & $\mathbf{0 . 7 6 1}$ & 0.035 \\
Disinhibition & $\mathbf{0 . 8 5 1}$ & 0.051 & 0.064 \\
Irritability & $\mathbf{0 . 6 0 1}$ & 0.362 & 0.201 \\
Aberrant motor behaviour & $\mathbf{0 . 4 4 9}$ & 0.405 & 0.137 \\
Night-time behaviour disturbances & 0.040 & $\mathbf{0 . 4 8 6}$ & 0.466 \\
Appetite and eating abnormalities & 0.097 & $\mathbf{0 . 6 2 9}$ & -0.040 \\
Eigenvalues & 3.544 & 1.739 & 1.329 \\
\% of Variance & 29.54 & 14.49 & 11.08 \\
\hline
\end{tabular}

External validation of the factor 'mood/apathy' was examined by calculating the Spearman's rank correlation between this factor and the score on the Cornell Scale for Depression in Dementia. This correlation was 0.79 ( $p<0.05)$, which can be regarded as high. The correlation with the factors 'hyperactivity' and 'psychosis' was $0.43(p<0.05)$ and 
0.29 ( $p<0.05)$, respectively. These correlations, although significant, were much lower than the correlation with the factor 'mood/apathy'. Therefore, the correlation was specific for the factor 'mood/apathy'.

The descriptions of the three factors and the separate symptom 'anxiety' are given in table 4. The factor scores are based on the summed NPI scores for each factor. The factor score for 'psychosis' was based on the summed score for delusions and hallucinations. Most patients ( $n=181$ ) had a score of 1 or more for at least one behavioural sub syndrome. Only 18 patients did not have any behavioural sub syndrome. The most common sub syndrome was the 'mood/apathy' sub syndrome (80.4\%), followed by 'hyperactivity' (60\%), 'anxiety' (almost 40\%), and 'psychosis' (37\%).

The patients with the 'psychosis' sub syndrome had the highest mean NPI total score. The combination of the sub syndromes 'mood/apathy' and 'hyperactivity' was present in 53\% of the patients. Mood and psychosis were present in $32 \%$ of the patients, and $26 \%$ of the patients had a score of 1 or more on both hyperactivity and psychosis. Anxiety most often co-occurred with the sub syndrome 'mood/apathy'.

Table 4. Description of the three NPI factors and anxiety

\begin{tabular}{llll}
\hline Outcome & Factor score & $\begin{array}{l}\text { Patients in factor } \\
\text { (frequency * severity }>0)\end{array}$ & $\begin{array}{l}\text { NPI total score } \\
\text { For patients in factor }\end{array}$ \\
\hline Hyperactivity factor & $7.0(0-54)$ & $119(59.8)$ & Mean (range) \\
Mood/apathy factor & $9.7(0-45)$ & $160(80.4)$ & $29.4(1-128)$ \\
Psychosis factor & $2.8(0-24)$ & $73(36.7)$ & $25.4(1-128)$ \\
Anxiety & $2.0(0-12)$ & $78(39.2)$ & $33.7(2-128)$ \\
No symptoms & & $18(9.0)$ & $29.2(1-128)$ \\
\hline
\end{tabular}

\section{Discussion}

The main finding of our study was that factor analysis indicated the presence of three behavioural sub syndromes 'mood/apathy', 'psychosis' and 'hyperactive behaviour'. Most symptoms had high factor loadings and give support to the differentiation between the three sub syndromes. The majority of patients had at least one sub syndrome. 'Mood/apathy' was the most common and co-occurred very often with 'hyperactivity'. 
The sub syndrome 'psychosis' was less prevalent and was associated with more severe total behavioural problems.

The attribution of the symptoms aberrant motor behaviour, night-time behaviour disturbances and anxiety to one of the sub syndromes can be argued. In this study, aberrant motor behaviour had high loadings for the sub syndromes 'hyperactivity' and 'mood/apathy', and thus could not be used to distinguish between the two sub syndromes. Likewise, night-time behaviour disturbances and anxiety had high loadings for the sub syndromes 'mood/apathy' and 'psychosis'. The internal consistency of the three sub syndromes was sufficient. However, because of a substantial improvement of Cronbach's alpha for the 'psychosis' sub syndrome when 'anxiety' was omitted, we decided that anxiety can best be regarded as a separate symptom, although it can cooccur with different sub syndromes.

The presence of the three ambiguous symptoms aberrant motor behaviour, anxiety, and night-time behaviour disturbances seems to be dependent on the severity of dementia, whereas the other nine symptoms were consistently attributed to the sub syndromes regardless of disease severity. However, because the three ambiguous symptoms were not able to clearly differentiate between the different sub syndromes in the total group, it cannot necessarily be concluded that the three symptoms are dementia stage specific. This deserves further study.

Because the presence of behavioural sub syndromes in a dementia population in need of care was our main point of interest, the factor analysis was performed on the etiologically heterogeneous group. However we are aware that behavioural syndromes may differ among patients with different etiologies. For example, hallucinations and delusions are more common in dementia with Lewy bodies and disinhibition in frontal dementia. Posthoc analyses were performed on Alzheimer patients, vascular dementia patients and other etiologies, but because of sample size and power it was only possible to perform a separate analysis for the Alzheimer patients. This resulted in a factor structure analogous to that found for the total patient group, and in particular resembled that of the mild dementia stages. Future studies must pay attention to the generalization of the sub syndromes to other dementia etiologies.

The three sub syndromes have face validity. In clinical practice the distinction between mood disorders, psychosis, and agitated behaviours is recognizable. The high correlation between the sub syndrome 'mood/apathy' and the Cornell Scale for Depression in Dementia also provided evidence for external validity. There was some instability of the symptoms in all three sub syndromes; however, the internal stability of the sub 
syndromes was satisfactory. Furthermore, each sub syndrome was characterized by two or more stable symptoms.

The factor structure of this study is consistent with that found in other studies. The factor we found to represent mood/apathy corresponds to factors proposed by other authors in factor analytic studies (Frisoni et al. 1999; Hope 1997; McShane 2000). Frisoni and colleagues (1999) found a sub syndrome 'mood' including depression and anxiety. Hope et al.(1997), using the Present Behavioural Examination (PBE) segregated depression as a separate factor. McShane et al. (2000), also using the PBE, found a factor depression, including anxiety. The finding of the factors hyperactivity and psychosis also seem to be consistent because in some kind the studies mentioned above agreed on the existence of these two factors. Frisoni et al. (1999) found a frontal and psychosis sub syndrome resembling our hyperactivity and psychosis sub syndromes, respectively. Hope et al. (1997) and McShane et al. (2000) also found a factor representing hyperactivity. Devenand and colleagues (1992a) found a disinhibition sub syndrome that has similarities with the 'hyperactivity' sub syndrome of our study.

There were also some differences between our results and those of previous studies. Fuh and colleagues (2001), using the NPI, found three factors. The first combined mood and psychosis, the second factor 'psychomotor regulation' resembled our hyperactivity factor, and the third factor represented 'social engagement' and included apathy and disinhibition. Hope et al. (1997) and McShane et al. (2000) distinguished aggressive behaviour as a separate sub syndrome. McShane and colleagues also did not find a factor 'psychosis'. The factor psychosis found in the study of Frisoni et al. (1999) included symptoms of our factor 'psychosis' and symptoms of our factor 'hyperactivity'.

The differences between studies may be related to the use of different measures, designs, and relatively small number of included patients for factor analytic purposes.

Frisoni et al. (1999)and Fuh et al. (2001) also studied the presence of sub syndromes of the NPI, but Fuh et al. used a very small sample (95 patients) for their factor analysis and Frisoni used the original 10-item version of the NPI. We performed a factor analysis of our data for the 10-item version of the NPI to determine whether the difference in factor structure reported by Frisoni et al. and that found in our study was the result of using different versions of the NPI. The same sub syndromes were found as, when the current 12-item version was used.

Lyketsos et al. (2001a; 2001b) performed a latent class analysis to identify symptom patterns which co-occur in individual Alzheimer patients, using a population-based study. 
This resulted into the classification of patients into three groups based on their neuropsychiatric symptom profile: an affective syndrome, a psychotic syndrome and a group with no neuropsychiatric symptoms or with a mono-symptomatic disturbance. The first two mentioned syndromes broadly resembled respectively our mood/apathy and psychosis sub syndrome. However, they did not find a group of patients with having predominantly agitated behaviours, like the hyperactive sub syndrome in our study. Agitation was regarded as a non-specific symptom. But the results reinforce the likely existence of behavioural sub syndromes in dementia patients, as well as in the population as in a clinical population.

When interpreting and using sub syndromes of dementia, one has to realize that differences can arise after controlling for, for example, stage of illness, sex, diagnosis, etc. Furthermore, the inclusion of rare variables with little variance in the analysis can affect the factor structure, especially for those factors with low eigenvalues (McShane 2000). This may have been the case for the symptom 'night-time behaviour disturbances' in our study. Overall it can be concluded that there are behavioural sub syndromes in dementia. Neurobiological correlates of behavioural symptoms in dementia have been found which support the existence of behavioural sub syndromes (Cummings 1997; Mega 2000; Sultzer 1996; Sultzer et al. 1995; Sultzer 1995; Zubenko et al. 1991).

This study had some limitations. For this study we applied a cross-sectional design without including data on the course of the sub syndromes; however, when follow-up data become available, it will be possible to examine the stability of the sub syndromes over time. In addition, the NPI is an observer-rated instrument. This can lead to information bias if the caregiver lacks or distorts information. However, it is generally acknowledged that behavioural problems in dementia are best assessed by interviewing informants who know the subject well (Zarit 1996).

Our study provides additional evidence of the existence of behavioural sub syndromes in dementia. The three sub syndromes can give insight into possible relationships between behavioural symptoms and their underlying cause and risk factors. The reduction of symptoms into sub syndromes can lead to greater ease in finding associations between behavioural disturbances and correlations with clinical variables (Frisoni et al. 1999). Furthermore, interventions may be more effective when targeting sub syndromes, rather than individual symptoms. However some caution is in order here. For example, data suggest an overlapping cholinergic-sensitive pathophysiology linking delusions and hallucinations, but these data also highlight potential differences in these symptoms with respect to differential response patterns to cholinergic treatment (Kaufer et al. 1998). 


\section{Key points}

- Three behavioural sub syndromes are identified: mood/apathy, psychosis, and hyperactivity

- Anxiety is regarded as a separate symptom

- The mood/apathy syndrome occurs in $80 \%$, hyperactivity in $60 \%$, and psychosis in $37 \%$ 
Chapter 3. Factor analysis of the Neuropsychiatric Inventory (NPI) 


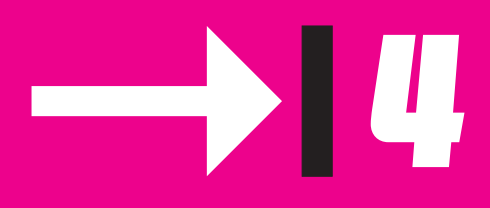

\section{Salivary cortisol patterns in caregivers}




\section{Abstract}

Objectives: The main purpose of the present study was to examine salivary cortisol patterns in caregivers of dementia patients and patients' behavioural and psychological symptoms (BPSD).

Methods: Diurnal cortisol profiles and response to awakening were measured in 57 caregivers and 55 non-caregiver controls. Cortisol was assessed from saliva collected with a cotton dental roll. Behavioural problems were assessed with the NeuroPsychiatric Inventory (NPI).

Results: Caregivers showed significantly higher levels of cortisol at the time of morning awakening than controls, with a smaller increase after awakening. Higher morning cortisol levels were found in caregivers of patients with high vs. low levels of BPSD; this effect was mediated by greater distress experienced by caregivers in relation to the BPSD.

Conclusions: These data suggest that high levels of behavioural problems in dementia patients influence the physiological stress response in caregivers. The observed elevation of morning cortisol levels could predispose caregivers to negative health consequences, with caregivers of patients with high levels of BPSD at even greater risk. 


\section{Introduction}

The behavioural and psychological symptoms of dementia (BPSD) exert a major influence on the quality of life of patients and their caregivers. They are reported by caregivers as difficult symptoms to manage and as a major source of stress (Donaldson et al. 1998; Draper et al. 1995; Gonzalez-Salvador et al. 1999). Several studies have demonstrated that patient behavioural problems are stronger predictors of caregiver distress than patient cognitive or functional impairment (Deimling and Bass 1986; Donaldson et al. 1998; Gaugler et al. 2000b; Kaufer 1998; Pruchno and Resch 1989; Vugt et al. 2003). However, despite clear evidence of subjective stress, little information is available on the impact of BPSD on physiological stress responses in caregivers.

One of the most extensively studied physiological responses to stress is change in the activity of the hypothalamic-pituitary-adrenocortical (HPA) axis, as reflected in cortisol secretion (Kirschbaum and Hellhamer 1994). Chronic stress can lead to either increases or decreases in overall cortisol levels (Preussner and Hellhamer 1999). Because cortisol has a pronounced circadian rhythm, with high levels in the morning and a decline in levels over the day, diurnal cortisol profiles provide a better picture of HPA activity than measurements at a single point in time. Another measure of HPA activity that may be a useful tool in stress assessment is the cortisol response to morning awakening (Preussner et al. 1997; Schmidt-Reinwald et al. 1999; Schulz et al. 1998; Wust et al. 2000b). Cortisol increases rapidly after awakening, with peak levels observed after 30-40 minutes. Chronic stress appears to influence the magnitude of this response, although it is not yet clear in which direction. Increased morning cortisol responses were found in individuals experiencing chronic work overload (Schulz et al. 1998); in contrast, blunted cortisol responses to awakening have been reported in individuals with burn-out (Preussner and Hellhamer 1999). Higher cortisol awakening responses have recently been reported in association with mild depressive symptoms (Preussner et al. 2003). Other factors that can influence the awakening response include awakening time, health status, age and sleep quality (Kudielka et al. 2003; Nicolson and van Diest 2000).

A number of studies have investigated the effects of chronic stress on HPA activity in caregivers of dementia patients. Of these, most reported elevated daytime cortisol levels in caregivers compared to healthy controls (Bauer et al. 2000; Cacioppo et al. 2000; Da Roza Davis and Cowen 2001; Vedhara et al. 1999), but two found no difference in cortisol levels (Irwin et al. 1997; Mills et al. 1997). One study found that cortisol levels in caregivers were associated with self-reported distress (Tarrier et al. 2002). Five studies assessed salivary cortisol concentrations (Bauer et al. 2000; Cacioppo et al. 2000; Da Roza Davis and 
Cowen 2001; Tarrier et al. 2002; Vedhara et al. 1999), and two studies used blood samples to measure plasma cortisol levels. All studies assessed only day-time cortisol levels (in some cases based on only 2 samples) and not the awakening response in caregivers. Five studies included morning cortisol levels (Cacioppo et al. 2000; Da Roza Davis and Cowen 2001; Irwin et al. 1997; Tarrier et al. 2002; Vedhara et al. 1999) but none of these studies assessed cortisol levels with reference to time of awakening. Measurement of morning cortisol levels with strict reference to time of awakening is extremely important in obtaining reliable results. In contrast, cortisol levels measured at fixed times in the morning shows very high intra-individual variability (Coste et al. 1994). Finally, there has been no attempt to date to relate individual differences in cortisol levels to patient functioning as a potential source of chronic stress in caregivers.

The primary goal of the present study was to determine whether BPSD is associated with physiological stress responses in primary caregivers. First, the hypothesis that diurnal levels of salivary cortisol and the cortisol awakening response differ between caregivers and non-caregivers was investigated. Secondly, we examined the influence of behavioural and psychological symptoms in dementia patients on cortisol measures in their caregivers.

\section{Methods}

\section{Subjects}

Participants were 57 primary caregivers of dementia patients and 55 non-caregiver control volunteers. The caregivers were participants in the Maastricht Study of Behaviour in Dementia (MAASBED) (Aalten et al. 2003), a two-year follow-up study of the course and risk factors for BPSD. Patients were in treatment at the Memory Clinic of the Academic Hospital Maastricht, or the geriatric division of the Regional Institute for Community Mental Health (RIAGG), Maastricht, the Netherlands. Caregivers were included if they were the primary caregiver and had contact with the patient at least once a week. Noncaregiver controls, matched with caregivers on sex, age and education, were recruited by telephone from a pool of volunteers for previous studies. Caregivers and controls who used medication that could influence cortisol levels were excluded (i.e., steroids, lithium, antidepressants, antipsychotics, and carbamezapine). To exclude somatic conditions that could affect cortisol levels, caregivers and controls were asked if they had any (history of) chronic health problems, if they had visited their general practitioner or a specialist for these health problems, and if they had received treatment for it in the past or were 
currently under treatment by a GP or specialist. The study was approved by the Hospital Medical Ethics Committee, and informed consent was obtained from all subjects.

Of the caregivers, 28 were spouses, 26 were children and 3 were other family members or close friends. Mean length of caregiving was 31.1 months (range 3-120), with a mean contact time per week of 85.6 hours (range 2-168).

Patients were diagnosed as having Alzheimer's disease $(n=46)$, vascular dementia $(n=7)$, frontal lobe dementia $(n=1)$, Parkinson's disease $(n=1)$, or mixed dementia $(n=2)$. There were 21 male and 36 female patients, with a mean age of 76.2 (range 54-96) and a mean Mini Mental State Examination score of 18.0 (range 5-27).

Caregiver and control groups had similar percentages of smokers ( 10 out of 57 versus 12 out of 55 respectively $(Z=-1.2 ; p=0.229)$. Alcohol consumption ranged from 0 to 4 glasses per day, with no significant difference between the groups $(Z=-1.1 ; p=0.285)$.

\section{Saliva collection}

Cortisol was assessed from saliva collected with a cotton dental roll, stored in a capped plastic vial (Salivette; Sarstedt, Etten-Leur, the Netherlands). Salivary cortisol correlates well with free plasma cortisol (Kirschbaum and Hellhamer 1994). In contrast to serum samples, saliva collection is non-invasive and interferes only minimally with normal daily routines. Subjects were given both oral and written instructions for collecting samples at home. Saliva samples were obtained: directly after awakening, 30 minutes after awakening, at 4 p.m., and at 9 p.m. . Subjects were free to use an alarm clock or to wake spontaneously. They were instructed not to eat, drink, or smoke in the 30 minutes before a saliva collection and to record the exact collection time.

\section{Patient measures}

BPSD was measured with the NeuroPsychiatric Inventory (NPI) (Cummings 1994), a structured interview with the caregiver that evaluates 12 behavioural and psychological symptoms in patients. The total score on each item can range from 1 to 15, obtained by multiplying severity (1 "mild" to 3 "severe") by frequency (1 "sometimes" to 5 "very often").

\section{Caregiver measures}

For each BPSD symptom on the NPI, caregivers rated the level of distress they experienced on a scale from 0 (none) to 5 (extreme). NPI-D score is the sum of these 12 ratings. 
Physical health complaints were measured with the physical functioning subscale of the RAND-36 questionnaire (Van der Zee and Sanderman 1993). Ten items are rated on 3point scales ranging from severely impaired to not at all impaired, with higher scores representing less impairment.

\section{Caregiver and control measures}

Overall subjective stress was measured with the Perceived Stress Scale (PSS; 10-item version) (Cohen and Williamson 1988). This questionnaire is a global measure of the degree to which situations in one's life in the past month are appraised as stressful. Items are rated on a 5-point scale ranging from 0 (never) to 4 (very often).

The Symptom-Checklist 90 (SCL-90) (Arrindell and Ettema 1986) total score was used as a measure of general psychological distress.

We used the Montgomery-Asberg Depression Rating Scale (MADRS) (Montgomery and Asberg 1979), a structured interview, to measure depressive symptoms. The MADRS contains relatively few somatic items, which makes this instrument suitable for a wide age range including elderly subjects. Ratings (from 0 to 6 ) on the 10 items were summed.

Caregivers and controls were asked to report any physical health problems and habitual mean hours of sleep per night. Sleep problems were assessed with three items from the Hamilton Depression Rating Scale (HDRS) (Hamilton 1960), rated on a three point scale (from 0 to 2 ) and then summed.

\section{Biochemical analysis}

Uncentrifuged saliva samples were frozen at $-20^{\circ} \mathrm{C}$ until analysis. Cortisol levels were determined in duplicate by direct radioimmunoassay, using ${ }^{125}$ iodohistamine-cortisol and anti-cortisol serum made against the 3-CMO-BSA conjugate (Dr. J. Sulon, University of Liège, Belgium). After overnight incubation at $4^{\circ} \mathrm{C}$, separation of free and antibody-bound ${ }^{125}$-cortisol was performed by a conventional "second antibody" method. The lower detection limit of the assay was $0.20 \mathrm{nmol} / \mathrm{L}$; the standard curve ranged up to $110 \mathrm{nmol} / \mathrm{L}$. Mean intra- and interassay coefficients of variation were $4.3 \%$ and $8.3 \%$, respectively.

\section{Data analysis}

Cortisol values were logarithmically transformed to normalize distributions. One control subject was excluded because cortisol levels were far outside the normal range (> 55.2 $\mathrm{nmol} / \mathrm{l})$. Five subjects (1 control, 4 caregivers) were excluded from analyses of awakening 
response due to deviant ( $<15$ min., $>45$ min.) or unreported time intervals between samples 1 and 2 .

The awakening response was defined as the change in cortisol level from the first to the second sample (directly after awakening and 30 minutes later). Cortisol measures directly after waking up, at 4 p.m. and at 9 p.m. were standardized within time of day and averaged to compute daily average cortisol (DAC) (Gunnar et al. 2001).

Univariate comparisons were performed with Student t-test, Chi Square test and MannWhitney $U$ test. Differences in diurnal cortisol profiles between caregivers and control subjects were analysed using repeated measures analysis of covariance (ANCOVA) with 'group' (caregivers versus controls) and sex as between-subjects factor and 'time' (timing of saliva samples: 1, 3 and 4) as within-subject factor; age and time (hours since midnight) of the first sample (awakening) were covariates. To examine the awakening response, this analysis was repeated for the cortisol measures at awakening and 30 minutes later. A forced entry regression analysis was performed to predict the cortisol awakening response by group, depressive symptoms, perceived stress, sleep problems and baseline cortisol levels.

In a separate analysis, caregivers were assigned to a 'low BPSD' and a 'high BPSD' group, according to a median-split on BPSD total scores. Differences in cortisol levels between these two groups were analysed, as above, with repeated measures ANCOVA, this time with BPSD ('high' versus 'low') as between-subjects factor. Greenhouse-Geisser corrections were applied when appropriate. Finally, a forced entry regression analysis was performed to predict the cortisol awakening response by group, caregiver distress (NPI-D), physical health problems (RAND-36), depressive feelings (MADRS) and hours of sleep. Significance was tested with two-tailed tests, with $\alpha=.05$

\section{Results}

\section{Group characteristics}

Caregiver and control characteristics are shown in table 1. There were no significant differences between the two groups in age, education, or sex distribution. However, caregivers reported significantly more subjective stress (PSS) and more depressive symptoms (MADRS) than the control subjects. No differences were found in general psychological distress (SCL-90), habitual sleep hours, and sleep problems. Awakening time on the sampling day ranged from 0410 to $1020 \mathrm{~h}$ and did not differ between controls and caregivers $(t=0.04 ; \mathrm{df}=108 ; \mathrm{p}=0.967)$. 
Table 1. Differences between caregiver and control groups

\begin{tabular}{lllll}
\hline & $\begin{array}{l}\text { Caregivers } \\
\mathrm{n}=57\end{array}$ & $\begin{array}{l}\text { Controls } \\
\mathrm{n}=55\end{array}$ & Test value & P value \\
& mean (range) & mean (range) & & \\
\hline Age (years) & $60.4(34-81)$ & $60.5(31-85)$ & $\mathrm{t}=-0.04$ & 0.968 \\
Education $^{1}(1-8)$ & $3.6(1-8)$ & $3.7(1-8)$ & $\mathrm{t}=-0.40$ & 0.688 \\
Sex $^{2}$ (m/f) & $21 / 36$ & $19 / 36$ & $\chi 2=0.06$ & 0.800 \\
PSS & $15.3(4-30)$ & $12.3(0-26)$ & $\mathrm{t}=2.67$ & 0.009 \\
MADRS & $7.4(0-23)$ & $3.4(0-11)$ & $\mathrm{Z}=-3.72$ & $<0.001$ \\
SCL-90 & $134.6(91-236)$ & $129.3(90-225)$ & $\mathrm{t}=0.78$ & 0.440 \\
Sleep hours & $7.2(3.5-9.5)$ & $7.1(4-10)$ & $\mathrm{t}=0.54$ & 0.592 \\
Sleep problems & $1.2(0-4)$ & $1.0(0-6)$ & $\mathrm{Z}=-0.98$ & 0.325 \\
Health problems $^{3}(\mathrm{Y} / \mathrm{N})$ & $32 / 24$ & $25 / 28$ & $\chi 2=1.09$ & 0.297 \\
\hline
\end{tabular}

PSS = Perceived Stress Scale $\mid$ MADRS = Montgomery Asberg Rating Scale $\mid$ SCL-90 = Symptom Checklist-90

1. ranging from primary school to university degree $\mid 2$. values represent number of males and females $\mid 3$. values represent number of subjects with or without health problems

\section{Differences between caregivers and controls in cortisol patterns}

ANCOVA results showed a significant main effect of time $(F=6.0(1.8,184.5), p=0.004)$, and a time by group interaction effect $(F=6.9(1.8,184.5), P=0.002)$. In general, men had higher cortisol levels than women $(F=4.7,(1,100), p=0.033)$. There were no significant main effects of group, age or time of awakening. As shown in Figure 1, cortisol levels were high in the morning and declined over the day in both groups. Post-hoc comparisons revealed significantly higher cortisol levels for caregivers directly after waking up $(t=3.51, \mathrm{df}=102$, $p=0.001)$

For the cortisol awakening response, ANCOVA results showed no main effect of time, but a significant time by group interaction effect $(F=7.3(1,96), p=0.008)$ as well as a significant main effect of group ( $F=4.5(1,96), p=0.036)$, indicating that the cortisol increase after awakening differed between groups, with caregivers having higher overall cortisol levels in the morning and a smaller cortisol awakening response. There were no significant effects of sex, age or time of awakening. Higher cortisol levels at awakening were related to an attenuated subsequent response $(r=-0.58, p<0.001)$.

To explore the association between group differences in the cortisol response to awakening and differences in perceived stress, depressive symptoms and sleep hours, a two-step forced entry regression analysis was performed with the cortisol awakening response as dependent variable and group as predictor in the first step, adjusted for time of awakening. In the second step depressive symptoms, perceived stress, sleep hours and 
baseline cortisol levels were also entered in the analysis as predictor variables. In the first step, group was a significant predictor of the cortisol response $(t=2.1, p=0.033$ ). However, in the second step group was no longer significant $(t=0.7, p=0.452)$ and only baseline cortisol level was a significant predictor of the cortisol awakening response $(t=-$ $6.4, p \leq 0.001)$. Pearson correlation showed no independent association between the cortisol awakening response and depressive symptoms $(r=-0.02, p=0.827)$ or perceived stress $(r=0.04, p=0.717)$.

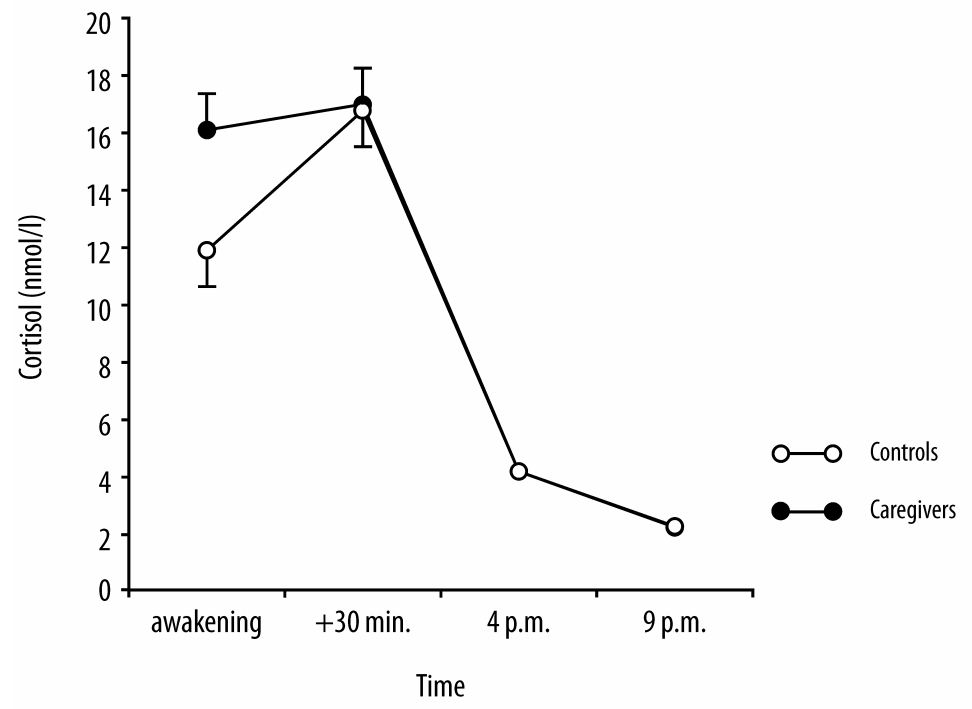

Figure 1. Diurnal cortisol levels (mean \pm SEM) in caregivers $(n=50)$ and controls $(n=51)$

\section{Association between BPSD and cortisol patterns in caregiver}

Caregivers who reported many behavioural problems in the patient ('high BPSD') were compared with those who reported fewer behavioural problems ('low BPSD'). The groups did not differ in the nature of the relationship between caregiver and patient (spouse vs. offspring $\chi^{2}=2.11, d f=1, p=0.189$ ), length of caregiving ( $\left.t=-0.05, d f=55, p=0.964\right)$, or contact hours per week $(t=1.14, d f=55, p=0.259)$. High BPSD caregivers reported significantly more distress related to patients' symptoms (Mann Whitney $\mathrm{U} ; \mathrm{z}=-5.47, \mathrm{p}<$ 0.001), more physical health complaints (Mann Whitney $U ; Z=-2.62, p=0.009$ ) and greater perceived stress $(t=-2.11, d f=53, p=0.040$ ). The two groups did not differ in depressive symptoms (Mann Whitney $\mathrm{U} ; \mathrm{z}=-0.57, \mathrm{p}=0.570$ ) or sleep hours $(t=1.48, \mathrm{df}=$ $54, p=0.145$ ). 
To examine whether differences in cortisol levels in caregivers were related to behavioural problems in the patient, diurnal cortisol levels at awakening, 4 p.m., and 9 p.m. were compared in the high versus low BPSD groups (see Fig. 2). ANCOVA results showed a main effect of sampling time only $(F=3.3(2,96), p=0.040)$.

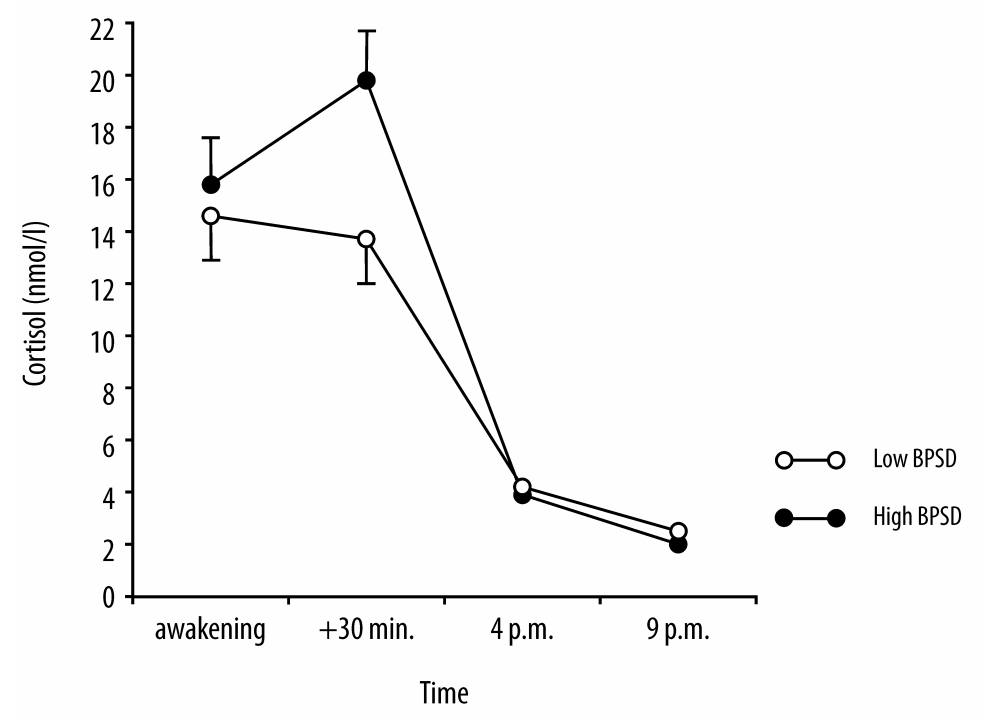

Figure 2. Diurnal cortisol levels (mean \pm SEM) in caregivers reporting high BPSD ( $n=27)$ and caregivers reporting low BPSD ( $n=23)$

With respect to the cortisol awakening response, results showed a significant time by group interaction effect $\left(F=4.7_{(1,46),} p=0.035\right)$, with a larger response in caregivers in the high BPSD group. There were no main effects of sampling time, group, sex, age or time of awakening. Post-hoc comparison at the two sampling times revealed significantly higher cortisol levels for caregivers in the high BPSD group 30 minutes after waking $(t=-2.5, \mathrm{df}=$ $50, p=0.017)$.

To test the hypothesis that caregiver distress mediates the association between BPSD and the cortisol awakening response, a two-step forced entry regression analysis was performed with the cortisol awakening response as dependent variable and group (high versus low BPSD) as predictor in the first step, adjusted for time of awakening. In the second step distress related to behavioural problems in the patient (NPI-D), physical health complaints (RAND 36 subscale), depressive symptoms (MADRS), hours of sleep and 
baseline cortisol levels were also entered in the analysis as predictor variables. SCL-90 scores were not included in the analysis because of a high correlation between the SCL90 and MADRS $(r=0.72, p<0.001)$. Of the variables in the analysis, baseline cortisol levels $(t=-5.2, p \leq 0.001)$ and distress related to behavioural problems $(t=2.0, p=0.050)$ were significant predictors, with lower baseline cortisol levels and greater distress associated with larger responses.

\section{Discussion}

The present study supports the hypothesis that salivary cortisol patterns change in relation to the stress of caregiving. This was particularly true for morning measures. Caregivers showed significantly higher levels of cortisol at the time of morning awakening than controls, with a smaller increase after awakening. In addition, in the caregiver group, a higher cortisol awakening response was found in caregivers of patients with high levels of BPSD. Caregiver distress related to problem behaviours mediated the relationship between BPSD and the cortisol awakening response. These findings provide evidence for physiological indicators of chronic stress in caregivers, in addition to subjective measures. Elevated morning cortisol levels in caregivers indicate an increased HPA activity, in line with several previous investigations (Bauer et al. 2000; Da Roza Davis and Cowen 2001; Vedhara et al. 1999). To our knowledge, this is the first study to examine the cortisol awakening response in dementia caregivers. We found elevated cortisol levels in caregivers directly after waking up, associated with blunted subsequent response compared to controls. The observed pattern is consistent with previous findings of blunted cortisol responses to awakening in teachers reporting high levels of burnout and may reflect an inability to cope with prolonged stress (Preussner and Hellhamer 1999). It is important to note, however, that these diminished cortisol responses to awakening were associated with higher initial hormone levels, as has also been found in other studies (Kudielka and Kirschbaum 2003; Schmidt-Reinwald et al. 1999). In addition, we found no significant relationship between the cortisol response to awakening and group differences in perceived stress and depression. We speculate that cortisol awakening responses in caregivers may better be explained by more specific aspects off stress within the caregiving situation, instead of global measures of distress.

In addition, we investigated differences in cortisol levels in caregivers in relation to BPSD, a potential source of stress. In this case, caregivers who reported high levels of BPSD displayed a higher morning cortisol awakening response than caregivers who reported low levels of BPSD. This association between BPSD and the cortisol awakening response is 
probably mediated by the emotional reaction of the caregiver to patient problem behaviours. Indeed, an additional analysis showed that the cortisol awakening response was specifically related to distress caused by BPSD and not to physical health complaints, depressive symptoms or hours of sleep. A greater cortisol response to awakening has also been found in individuals with chronic work overload (Wust et al. 2000b) or high perceived stress (Wust et al. 2000a). A slight non-significant decrease was found in cortisol after awakening in caregivers of low BPSD patients. This finding might be explained by the higher initial cortisol levels after awakening compared to normal controls due to the influence of chronic stress. Results from this study and findings of previous studies indicate that higher baseline cortisol levels are associated with blunted subsequent responses (Kudielka and Kirschbaum 2003; Schmidt-Reinwald et al. 1999). However, in the high BPSD group stress levels are even higher than in the low BPSD group, resulting in an additional increase in cortisol levels on top of the high cortisol baseline levels.

Some limitations should be considered. Although there were no differences between caregivers and controls in habitual sleep duration, we are unable to assess whether differences in sleep problems in the past night might have influenced cortisol levels the next morning. Secondly, sampling cortisol over two days instead of one, with more frequent samples per day, would have increased the reliability of individual diurnal cortisol profiles. In addition, the results should be interpreted cautiously, because sample collection was unsupervised. We excluded samples reportedly taken outside an acceptable time interval around the target time, with stricter criteria for the awakening response interval; this procedure improves the quality of the data obtained (Kunz-Ebrecht et al. 2004). However, recent studies in which saliva samples were monitored electronically (Broderick et al. 2004; Kudielka and Kirschbaum 2002) indicate that some subjects fail to report sampling times accurately; inclusion of samples taken later than reported can bias results, especially in the case of cortisol response to awakening.

The caregiver sample was heterogeneous, which may also have obscured subtle patterns. Both spouses and children, over a wide age range, with varying intensity and duration of caregiving activity, were included in the sample. However, caregivers in the high and low distress groups did not differ in the nature of the relationship between caregiver and patient, number of contact hours per week or months of care.

In conclusion, this study provides evidence for increased HPA activity in the morning in caregivers, as reflected by elevated salivary cortisol levels. With respect to the hypothesis that severity of patients' behavioural symptoms influences cortisol levels in caregivers, 
increased awakening responses were found in caregivers reporting high levels of BPSD in the patient, an effect that was mediated by caregiver distress due to these problems. The absence of significant associations between caregiver status and cortisol levels later in the day suggest that morning cortisol measures may be especially sensitive markers of this type of chronic stress (Kunz-Ebrecht et al. 2004), which should be taken into account in future research. Samples collected at the time of awakening also appear to provide a better estimate of 24 ht levels than samples collected at a fixed time (e.g., 8 a.m.) (Yehuda et al. 2003).

Previous reports have stated that increased HPA activity can lead to poorer immune function, and subsequent increased disease vulnerability (Bauer et al. 2000). The observed elevation of morning cortisol levels could predispose caregivers to negative health consequences, with caregivers of patients with high levels of BPSD at even greater risk. A recent study found for example that higher cortisol responses to awakening were associated with slower wound healing (Ebrecht et al. 2004). Longitudinal data from the MAASBED study will provide more information on both the causal relationship between chronic stress and cortisol secretory patterns and the longer-term consequences of stressrelated HPA abnormalities in dementia caregivers.

\section{Key points}

- $\quad$ Caregivers have higher morning cortisol levels than controls

- High levels of patient behavioural problems are related to high levels of morning cortisol in caregivers, mediated by caregiver stress 
Chapter 4. Salivary cortisol patterns in caregivers 


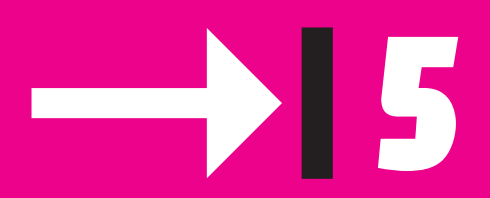

\section{Behavioural disturbances in dementia patients and quality of the marital relationship}




\section{Abstract}

Objectives: To investigate the relationship between behavioural problems in patients with dementia and changes in the marital relationship.

Methods: Fifty-three spouse caregivers of patients with dementia participated in the study. Questionnaires and interviews were used to examine caregiver perception of changes in the quality of their relationship. Behavioural disturbances in the patient were measured with the NeuroPsychiatric Inventory (NPI).

Results: Caregivers experienced a deterioration of their relationship, yet at the same time most felt closer to their spouse now than in the past. Regression analysis revealed that patient behavioural problems were, independent of patient cognitive status or functional impairment, associated with deterioration in the quality of the relationship between patient and caregiver. Patient apathy rather than depressive mood was associated with this deterioration. Apathy diminished the amount and reciprocity of interactions between partners.

Conclusions: These results show that passive behaviour rather than excessive behaviour has most impact on the deterioration of the marital relationship. Intervention programmes should target relationship problems when problem behaviour, especially apathy, is present in patients with dementia. 


\section{Introduction}

As dementia progresses, the marital relationship becomes increasingly imbalanced. Total relationship quality and the level of intimacy tend to diminish (Fearon et al. 1998; Morris et al. 1988; Wright 1991), communication becomes more difficult, and enjoyment of each other's companionship and reciprocity diminish (Gallagher-Thompson et al. 2001; Wright 1991). These changes in the marital relationship have a negative impact on depression and burden of care experienced by caregivers (Donaldson et al. 1998; Draper et al. 1995; Lawrence et al. 1998) and they increase the risk of institutionalisation (Spruytte et al. 2001).

The extent to which quality of the relationship changes, varies for different patientcaregiver couples. Researchers have found a negative correlation between BPSD and relationship quality, although the exact nature of this association is still unclear (Lawrence et al. 1998; Morris et al. 1988). It is well-known, however, that behavioural symptoms in the patient are important sources of stress and therefore may change the caregiver's feelings towards the patient. Studies which have touched on this subject have primarily focused on behavioural problems in general, although the extent to which BPSD is related to the quality of the marital relationship may vary for different symptoms or groups of symptoms.

In this study, we investigated whether changes in the quality of the marital relationship are related to psychological and behavioural symptoms in patients with dementia. In addition, the specific influence of different clusters of symptoms was examined. For this purpose, both quantitative and qualitative data were used.

\section{Methods}

\section{Subjects}

Sixty-four spouse caregivers of consecutively referred patients with dementia (DSM IV; American Psychiatric Association 1994) participated in this study. The present study is part of the Maastricht Study of Behaviour in Dementia (abbreviated as MAASBED). MAASBED is a 2-year follow-up study that focuses on the course and risk factors of behavioural and psychological symptoms of dementia (BPSD). The study population was drawn from the Maastricht Memory Clinic of the Academic Hospital Maastricht, and the geriatric division of the Regional Institute for Community Mental Health (RIAGG) of Maastricht, the 
Netherlands. All patients were living at home at baseline. Informed consent was obtained from all subjects.

Of the 64 spouse caregivers participating in MAASBED, 53 caregivers returned the questionnaires (82.8\%). Caregivers who filled out the questionnaires did not differ from those who did not in terms of sex, education, depression, burden, and patient behavioural problems. The caregivers who did not complete the questionnaires tended to be older $(p=0.051)$. Baseline data were used for this study. Subject characteristics are shown in table 1.

Table 1. Patient and caregiver characteristics

\begin{tabular}{lll}
\hline & $\begin{array}{l}\text { Caregiver } \\
(\mathrm{n}=53)\end{array}$ & $\begin{array}{l}\text { Patient } \\
(\mathrm{n}=53)\end{array}$ \\
\hline & Mean (SD) & Mean (SD) \\
\hline Sex' (m/f) & $22 / 31$ & $32 / 21$ \\
Age & $68.3(7.9)$ & $71.6(7.6)$ \\
Education ${ }^{2}$ (1-8) & $3.1(1.7)$ & $2.8(1.9)$ \\
Contact per week (hours) & $153.7(13.9)$ & \\
Duration of care (months) & $27.0(25.4)$ & \\
Duration of illness (months) & & $46.2(31.2)$ \\
MMSE-score & & $18.6(5.2)$ \\
Dementia diagnosis patient & & \\
& Alzheimer's disease & 41 \\
& Vascular dementia & 7 \\
& Frontal lobe dementia & 2 \\
& Parkinson's disease & 1 \\
& Primary Progressive aphasia & 1 \\
& Mixed (AD/vascular) & 1 \\
\hline
\end{tabular}

MMSE - Mini-Mental State examination

1. values represent number of males and females $\mid 2$. ranging from primary school (1) to university degree (8)

\section{Patient measures}

Patient behavioural problems were measured with the NeuroPsychiatric Inventory (NPI). The NPI (Cummings 1994) is a structured interview with the caregiver and evaluates 12 neuropsychiatric disturbances. The score of each item is obtained by multiplying severity (1-3) by frequency (1-4) (range 0-12). The level of distress that a particular symptom causes the caregiver is also rated (range 0-5). Principal component analysis was used to detect 
behavioural sub-syndromes. This factor analysis resulted in three factors (Aalten et al. 2003): (1) a hyperactivity factor including the symptoms disinhibition, irritability, agitation, euphoria, and aberrant motor behaviour (Cronbach's alpha =0.73); (2) a mood/apathy factor including night-time behaviour disturbances and eating abnormalities $(\alpha=0.63)$; and (3) a psychosis factor including hallucinations and delusions $(\alpha=0.72)$. Anxiety was not part of any factor and therefore was not included in the analysis. Factor scores are based on the summed observed NPI item scores for each factor.

The Mini Mental Status Examination (MMSE; Folstein et al. 1975) was used to measure patient cognitive functioning. The dependency of the patient in daily activities was measured with the Interview for Deterioration in Daily living activities in Dementia (IDDD; Teunisse 1995). This questionnaire consists of 20 items reflecting initiative to and actual performance of daily activities. We used a summed score for items concerning actual performance of activities (range 0 to 44).

\section{Caregiver measures}

First, the quality of the relationship was measured using four items of the University of Southern California Longitudinal Study of Three-Generation Families measures of positive affect (Lawrence et al. 1998). The items indicate a) general closeness, b) communication, c) similarity of views about life and d) degree of getting along. Answer categories range from 1 (not at all) to 4 (very). Spouses answered these questions in terms of the current situation (Cronbach's alpha $=0.73$ ) and to what degree the relationship had changed since illness onset $(1=$ much better, $5=$ much worse) $(\alpha=0.78)$. Summed scores were used as an index of the change in relationship quality.

Caregivers then rated their present and past relationship on two visual analogue scales ranging from 0 (not good at all) to 10 (very good). They also completed a semi-structured interview, administered according to a standardized procedure, to assess the quality of the relationship from their perspective. In short, caregivers were asked to describe their spouse and their relationship with their spouse in their own words. In an additional interview, spouse caregivers reported on changes in their relationship since the onset of the dementia. 


\section{Data analysis}

Multiple regression analysis was used to examine the relationship between dependent (relationship change) and independent variables (patient functioning). The potential influence of outliers on the results was checked according to standard procedures (Hair et al. 1988).

Qualitative interviews were analysed to assess the expected association between dependent (relationship change) and independent variables (patient functioning). BPSD and relationship change were dichotomised with a median split for this qualitative analysis. As the purpose of the qualitative analysis was to explore the impact of BPSD on changes in the relationship, the analysis was focused on couples that scored high on behavioural problems $(n=28)$. Nineteen of these couples (one caregiver refused to be interviewed) scored also high on relationship change and 9 couples (again one refusal) scored low on relationship change.

The semi-structured, open-ended interviews were tape-recorded and transcribed verbatim for analysis. The content of the transcripts was analysed using the framework approach to identify common themes and issues (Ritchie and Spencer 1994). This process involved clustering, selecting, and coding data on the basis of the thematic framework derived from the quantitative analysis and on issues raised by the respondents themselves. The process of interpreting and identifying categories involved searching and counting key themes, noting associations and comparing (deviant) cases. Reproducibility of the results was checked by an independent observer who came to the same conclusions in a set of interviews drawn at random from the total group. Ethnograph version 5 was used for qualitative analysis (Seidel 1998).

\section{Results}

\section{Relationship quality}

Most of the spouse caregivers rated the premorbid quality of their relationship very positively (mean 8.49). Nearly $90 \%$ rated their past relationship as 7 or higher on a scale from 1 (not good at all) to 10 (very good). The quality of the current relationship was rated significantly less positive (mean 7.35; Friedman test: $p=0.000$ ). Seventy per cent rated their present relationship as 7 or higher. Table 2 presents caregiver perception of the current quality of the relationship and of the relationship change. Most spouses reported the greatest deterioration to have occurred in communication with the patient, yet most 
spouses also reported feeling closer to the patient than in the past. They were less likely to report a change in the degree of getting along and having similar views about life.

Table 2. Percentage response per answer category to items of relationship quality $(\mathbf{n}=\mathbf{5 3})$

\begin{tabular}{lccc|ccc}
\hline & \multicolumn{6}{c}{ Answer categories (\%) } \\
\hline & \multicolumn{3}{c}{ Current relationship } & \multicolumn{3}{c}{ Relationship change } \\
\hline Items & $\begin{array}{c}\text { not at all } \\
\text { somewhat }\end{array}$ & fairly & very & $\begin{array}{c}\text { much better } \\
\text { better }\end{array}$ & same & $\begin{array}{c}\text { worse } \\
\text { much worse }\end{array}$ \\
\hline How close do you feel? & 7.5 & 22.6 & 69.8 & 47.2 & 39.6 & 13.2 \\
How is communication? & 54.7 & 39.6 & 5.7 & 9.4 & 28.3 & 62.2 \\
How similar are your views? & 45.3 & 41.5 & 13.2 & 15.1 & 52.8 & 32.1 \\
How well do you get & 9.4 & 35.8 & 54.7 & 11.3 & 56.6 & 32.1 \\
along? & & & & & & \\
\hline
\end{tabular}

\section{Changes in relationship quality associated with behavioural problems}

Overall, $90 \%$ of the patients with dementia had some behavioural symptoms (table 3). A regression analysis was performed to determine the degree to which behavioural problems predicted relationship change independent of cognitive status and functional impairment.

Table 3. Patient behavioural syndrome scores, number of patients with each syndrome, and mean NPI total scores $(\mathbf{n = 5 3 )}$

\begin{tabular}{llll}
\hline Syndromes & Syndrome score & $\begin{array}{l}\text { Patients with syndrome } \\
\text { score }>0^{2}\end{array}$ & $\begin{array}{l}\text { NPI total score for patients } \\
\text { per subsyndrome }\end{array}$ \\
\hline Mean (range) & $\mathrm{N}(\%)$ & Mean (range) \\
\hline Myperactivity & $5.3(0-27)$ & $30(56.6)$ & $25.7(1-68)$ \\
Mood/apathy & $8.6(0-35)$ & $43(81.1)$ & $20.9(1-68)$ \\
Psychosis & $1.6(0-21)$ & $11(20.8)$ & $30.5(5-68)$ \\
\hline
\end{tabular}

1. Computed as the sum of NPI item scores belonging to each subsyndrome | 2. Eight patients (13.9\%) had no symptoms | 3. Computed as NPI total scores for patients scoring on the subsyndrome

Cook's distance indicated that there were two major outliers with extremely high NPI scores. Exclusion of these outliers resulted in a more stable model and reduced the $\mathrm{p}$ value of NPI total from 0.068 to 0.001 . Behavioural problems were highly predictive of 
relationship change, whereas cognitive status and functional impairment were not (table 4). Sex was not a confounder.

A second regression analysis differentiated between the three behavioural syndromes (table 4). Mood/apathy was a significant predictor of relationship change, whereas hyperactivity and psychosis were not. Again, sex was not a confounder.

\section{Table 4. Multiple regression analysis predicting relationship change}

\begin{tabular}{lll}
\hline Independent variable & Unstandardised B coefficient & $P$ value \\
\hline $\mathrm{F}(3,47)=5.31, \mathrm{P}=0.003$ & & \\
$\mathrm{NPI}$ total & 0.103 & 0.001 \\
$\mathrm{MMSE}$ & $4.92 \mathrm{E}-02$ & 0.508 \\
$\mathrm{IDDD}$ & $7.89 \mathrm{E}-03$ & 0.832 \\
\hline $\mathrm{F}(3,47)=4.88, \mathrm{P}=0.005$ & & \\
Mood/apathy & 0.122 & 0.010 \\
Hyperactivity & $9.02 \mathrm{E}-02$ & 0.151 \\
Psychosis & $8.62 \mathrm{E}-02$ & 0.457 \\
\hline
\end{tabular}

$\mathrm{NPI}=$ Neuropsychiatric Inventory $\mid \mathrm{MMSE}=$ Mini Mental State Examination $\mid$ IDDD = Interview of Daily living activities in Dementia

\section{Exploration of the association between BPSD and relationship change}

Qualitative data were used to further assess the association between patient behavioural problems and deterioration of the marital relationship. Several key issues became apparent, the most evident being communication difficulties (mentioned by 16/18 caregivers), restriction of freedom (11/18), lack of intimacy (9/18), and diminished joint activities (9/18).

Changes in the relationship were examined in relation to BPSD in the group that had high scores for relationship change and behavioural problems. The most disruptive patient behavioural problems mentioned by caregivers (16/18) were apathy and withdrawal. These symptoms were mentioned by the caregivers as distressing and as having a negative impact on the relationship. Post-hoc regression analysis of the mood/apathy items as predictor for relationship change in the total group $(n=53)$ confirmed the finding that apathy was the only significant predictor $(B=0.356 ; p=0.014)$, whereas depression, night-time behaviour disturbances, and eating abnormalities were not significant predictors. 
Table 5. Interview quotations with theme and respondent number

\begin{tabular}{|c|c|}
\hline Quotations & Theme \\
\hline \multicolumn{2}{|l|}{ Caregiver group reporting high relationship change } \\
\hline $\begin{array}{l}\text { "It is awful to be around a man all day who doesn't } \\
\text { communicate. I rather talk to someone about unimportant } \\
\text { or stupid things, than not to talk at all » (\#4) }\end{array}$ & Communication \\
\hline $\begin{array}{l}\text { "I miss the love between us. That is the most important } \\
\text { problem. I miss his arm around me when I am cooking } \\
\text { dinner» (\#19) }\end{array}$ & Intimacy \\
\hline $\begin{array}{l}\text { "I would like to go on holiday with him like we used to do, } \\
\text { but he has no interest at all " (\#8) }\end{array}$ & Joint activities \\
\hline $\begin{array}{l}\text { "You are losing someone against your will, you can try } \\
\text { everything, but you get no response at all. That is very } \\
\text { difficult for me» (\#5) }\end{array}$ & Reciprocity \\
\hline \multicolumn{2}{|l|}{ Caregiver group reporting low relationship change } \\
\hline $\begin{array}{l}\text { "Sometimes he wants to know what kind of clothes he has } \\
\text { and then he takes everything out of the wardrobe. This can } \\
\text { happen two or three times a week and I have to put } \\
\text { everything back» (\#14) }\end{array}$ & $\begin{array}{l}\text { Active disturbed } \\
\text { behaviour }\end{array}$ \\
\hline $\begin{array}{l}\text { "We often go away together, for example every Thursday. } \\
\text { We often go out for dinner and have a good time»(\#23) }\end{array}$ & Joint activities \\
\hline $\begin{array}{l}\text { "Every evening I have a conversation with him. We have } \\
\text { always done that, but now I am more aware of it» (\#15) }\end{array}$ & Communication \\
\hline
\end{tabular}

Communication problems increased when there was a lack of spontaneous speech or response from the patient. These communication difficulties hindered the caregivers from sharing their thoughts and feelings with the patient, which affected the reciprocity of the relationship. Apathy in the patient affected feelings of companionship - caregivers missed sharing experiences and activities with their spouse. Joint activities declined because the patient was no longer interested or the caregiver derived no pleasure from activities with a spouse who hardly ever responded. The level of affection and sexual intimacy declined when the patient lost interest or rejected the caregiver. Some caregivers were no longer sexually interested in their partners because they felt their relationship and their spouse had changed too much. Overall, patients with passive disturbed behaviour were often difficult to reach, had lost interest in their environment, and showed few emotions. These 
symptoms diminished caregiver-patient contact and the mutuality of the relationship (see table 5, group 1).

In contrast, in the couples who scored high on behavioural problems and low on relationship change, the most problematic behaviour was 'active disturbed behaviour'. Most caregivers (5/8) reported agitation, irritability, paranoia, or verbal aggression on the part of the patient. Although these behavioural problems were distressing to the caregivers, the amount and mutuality of interaction between the spouses in this group was affected less than in the group with more passive disturbed patients (see table 5, group 2)

\section{Discussion}

The main finding of our study is that deterioration of relationship quality is specifically associated with the presence of behavioural problems, notably apathy, in patients with dementia. In addition, the results confirm previous findings showing that, overall, caregivers experience a deterioration of their relationship (Horowitz and Shindelman 1983; Morris et al. 1988; Wright 1991), yet at the same time most felt closer to their spouse now than in the past.

The fact that most caregivers reported a stronger emotional bond with their spouse is in accordance with other investigations that identified not only negative changes but also some positive experiences of caregivers after onset of dementia (Horowitz and Shindelman 1983; Murray et al. 1999; Spruytte et al. 2001). This finding may be explained in a variety of ways. First, both caregiver and patient come into the same situation where they have to deal with the diagnosis dementia. Sharing this difficult experience may strengthen their bond. Second, the quality of the relationship between caregiver and patient can no longer be taken for granted. This may increase caregivers' awareness of their affection for the patient and makes them enjoy more of the time they still have together (see table 5, respondent number 15). Finally, the increasing difficulty of dementia patients to manage their day-to-day life may elicit feelings of empathy in the caregiver and the need to protect their spouse. This can make them feel closer to their partner while daily interaction becomes more difficult.

In accordance with findings of several other studies (Lawrence et al. 1998; Morris et al. 1988), behavioural problems had a significant effect on relationship deterioration, 
whereas patient functional disability or cognitive problems seemed to be less of a problem to caregivers. Caregiver experience of a greater deterioration of the relationship was particularly associated with more patient symptoms of mood/apathy. Qualitative data, confirmed in a post-hoc quantitative analysis, suggested that not depressive mood but apathy was the main symptom associated with deterioration of the relationship. This is in line with other studies that showed apathetic and withdrawn behaviour to be most stressful to caregivers (Greene et al. 1982; Landes et al. 2001; LoGiudice et al. 1995). Apathy in the patient diminished joint activities and sharing experiences, and loss of these essential elements restricts mutuality in the marital relationship. There was not a significant association between hyperactivity or psychosis and changes in the relationship, indicating that passive behaviour rather than active disturbed behaviour has more impact on the marital relationship. Apparently, caregivers are better able to cope with excessive behaviour that disrupts interaction than they are with a decrease in interaction because of diminished conversation or disinterest on the part of the patient.

A pre-morbid difficult relationship has been found to increase the risk of deterioration in the quality of the relationship (Williamson and Shaffer 2001). However, in the present study this was not taken into account because only a few caregivers reported a low premorbid relationship quality. A possible explanation is that caregivers tend to idealize their past relationship when they are confronted with the problems they experience in the current situation.

Some limitations of this study need to be considered. First, the findings are based on baseline data, so the causality of the association was not determined, although the qualitative data in this study suggest that BPSD has an effect on the change in the quality of the relationship. Moreover, a low relationship quality may have a negative effect on the course of behavioural and psychological problems of the patient. So there might be a bidirectional pattern of causation. Second, it cannot be precluded that the lack of association of hyperactivity and psychosis with relationship quality was due to the low frequency of these syndromes. However, qualitative data confirmed the finding that the caregiver-patient relationship was affected less when active disturbed behaviour was present in the patient. Third, the study sample was a relatively young group of patients due to the selection of patients who were living at home with their partner. Therefore, one should be cautious in generalizing these results. 
In conclusion, the restriction of interaction due to patient apathy appeared to be an important factor in the deterioration of the marital relationship. Identification of these vulnerable couples provides important information for further refinement of intervention programmes. One should be alert to relationship problems when a patient has problem behaviours, especially apathy. Intervention programmes should provide specific counselling for caregivers to reinforce positive perceptions of their relationship and to increase reciprocity, and they should aim at early activation of patients with dementia to prevent the development of apathy. Available cholinesterase inhibitors are also relevant in this respect, given the fact that they also may influence these behavioural symptoms (Cummings 2000). This may improve the quality of life for both partners and reduce psychological morbidity in caregivers.

Whether the quality of the relationship has an effect on the course of behavioural and psychological problems of the patient needs further investigation. Longitudinal data of the MAASBED study will provide further insight into the causal nature of this association.

\section{Key points}

- $\quad$ Caregivers experience a deterioration of their relationship, yet at the same time feel closer to their spouse

- $\quad$ Negative relationship changes are related to patient behavioural problems, but not to cognitive status or functional impairment

- Apathy has most impact on the deterioration of the spousal relationship 


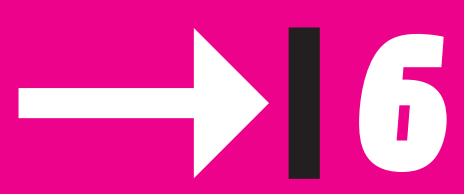

Impact of behavioural problems on spousal caregiverst a comparison between Alzheimer disease and Frontotemporal dementia 


\section{Abstract}

Objectives: Behavioural changes are a key factor in distinguishing Frontotemporal Dementia (FTD) from Alzheimer's Disease (AD); however, little is known about the impact of these changes on caregivers. The aim of this study was to compare caregivers' distress related to behavioural symptoms of AD and FTD.

Methods: Forty-seven spouse caregivers of consecutively referred patients with $A D$ and 27 spouse caregivers of patients with FTD participated in this study. Behavioural disturbances in the patient and caregivers' emotional reactions were measured with the NeuroPsychiatric Inventory (NPI).

Results: Patients with FTD had significantly higher levels of agitation, apathy, disinhibition and aberrant motor behaviour than did patients with AD. The caregivers of FTD patients reported disinhibition, depression and apathy as being severely distressing whereas the caregivers of AD patients reported patient apathy, depression and anxiety as being severely distressing. Higher mean distress scores were found for disinhibition and apathy in the FTD group. Furthermore, caregivers of FTD patients reported higher levels of general burden, and felt less competent than AD caregivers.

Conclusions: Caregivers of FTD patients were overall more distressed by the behaviour of their partners than were the caregivers of $A D$ patients. Findings from this study underscore the importance of differentiating between diagnostic groups when focusing on caregiver reactions to problem behaviour. 


\section{Introduction}

Behavioural and psychological symptoms are common in patients with dementia. These symptoms have important consequences for primary caregivers because they are associated with caregiver distress and negative health effects (Donaldson et al. 1998; Hooker et al. 2002). Moreover, these symptoms are important determinants of nursing home placement (O'Donell et al. 1992). A greater understanding of the impact of these symptoms on caregivers may facilitate the development of more specific caregiver interventions.

Research has identified a broad spectrum of behavioural disturbances related to dementia, including depression, apathy, agitation, aggressiveness and disinhibition. In addition to the well-known associations between caregiver distress and general measures of behavioural problems, some specific behavioural symptoms have been linked to caregiver burden. For example, apathetic and withdrawn behaviour are stressful to caregivers (Greene et al. 1982; Landes et al. 2001; LoGiudice et al. 1995; Vugt et al. 2003), as is disruptive behaviour (Deimling and Bass 1986). However, most of these studies looked at heterogeneous samples of dementia patients or included primarily patients with Alzheimer's disease (AD). Although a number of studies compared behaviour in different types of dementia, little is known about whether caregivers' emotional reactions to patient behavioural problems differ between specific diagnostic groups.

Behavioural changes are a key factor in distinguishing Frontotemporal dementia (FTD) from $A D$. At the onset of FTD, non-cognitive behavioural changes predominate and cognitive functions are relatively preserved, whereas the diagnosis of $A D$ is based principally on the presence of cognitive disturbances. Furthermore, unlike AD, FTD has a relatively early onset and there is early loss of insight (Perry and Miller 2001). A number of studies have investigated behavioural changes in FTD (Bathgate et al. 2001; Bozeat et al. 2000; Diehl and Kurz 2002; Levy et al. 1996; Rymer et al. 2002; Snowden et al. 2001), and it has been reported that FTD patients have overall more behavioural problems than AD patients (Levy et al. 1996). Symptoms that are more pronounced in FTD are apathy, disinhibition, euphoria, and aberrant motor behaviour.

In view of these differences in behaviour between the two disorders, we expected that caregiver distress related to the behavioural problems of the patient would differ between FTD and AD. Therefore, we compared the behavioural symptom profiles of AD and FTD patients, and the emotional distress of caregivers in relation to the behavioural symptoms of patients with $A D$ and FTD. 


\section{Methods}

\section{Subjects}

Forty-seven spouse caregivers of consecutively referred patients with AD (DSM IV) (American Psychiatric Association 1994) and 27 spouse caregivers of patients with FTD (diagnosis according the criteria of The Lund and Manchester Groups, 1994) participated in this study. Patients with $A D$ and their caregivers were drawn from the Maastricht Memory Clinic of the Academic Hospital Maastricht or the Department of Mental Health Care for the Elderly of the Community Mental Health Center of Maastricht, the Netherlands. They were included in the Maastricht Study of Behaviour in Dementia (MAASBED) (Aalten et al. 2003). MAASBED is a 2-year follow-up study that focuses on the course and risk factors of behavioural and psychological symptoms of dementia (BPSD). FTD patients and their caregivers were examined at the Department of Neurology of the Academic Hospital of Rotterdam, the Netherlands (Rosso et al. 2001).They were included in a follow-up study of caregiver burden in FTD. Baseline data were used for the current study. All patients were living at home at baseline. Informed consent was obtained from all subjects.

\section{Patient measures}

Patient behavioural problems were measured with the 10 item version of the Neuro Psychiatric Inventory (NPI). The NPI (Cummings 1994) is a structured interview held with the caregiver and evaluates 10 neuropsychiatric symptoms which are commonly observed in dementia: delusions, hallucinations, agitation / aggression, dysphoria / depression, anxiety, apathy, irritability, euphoria, disinhibition, and aberrant motor behaviour. The score for each item is obtained by multiplying severity (1-3) by frequency (1-4) (range 0-12). The validity and reliability of the NPI have been established earlier (Cummings and McPherson 2001), as well as its Dutch version (Kat et al., 2002).

The Mini Mental Status Examination (MMSE) (Folstein et al. 1975) was used to measure patient cognitive functioning. The dependency of the patient on assistance to perform daily activities was measured with the Interview for Deterioration in Daily living activities in Dementia (IDDD) (Teunisse and Derix 1991). This questionnaire consists of 20 items reflecting initiative to and actual performance of daily activities. A summed score was used for items concerning actual performance of activities (range 0 to 44) and for items concerning initiative to activities (range 0 to 36 ). 


\section{Caregiver measures}

For each of the 10 neuropsychiatric symptoms on the NPI, caregivers rated the level of distress they experienced on a scale from 0 (none) to 5 (extreme). NPI-D total score is the sum of these 10 ratings.

A visual analogue scale was used to assess overall caregiver burden, ranging from 1 ("not at all") to 10 ("extreme").

Caregiver competence was measured with the Short Sense of Competence Questionnaire (SOC) (Vernooij-Dassen et al. 1999). This questionnaire consists of 7 items rated on a 5point scale ( 1 "agree very strongly" to 5 "disagree very strongly"). These items reflect three domains of caregivers' feelings of being able to care for a demented person: (1) satisfaction with the demented person as a recipient of care; (2) satisfaction with one's own performance as a caregiver; (3) consequences of involvement in care for the personal life of the caregiver.

\section{Data analysis}

Demographic variables of the AD and the FTD group were compared using t-tests and Chi-square tests. Mean NPI symptom scores and NPI-distress scores for the FTD and AD groups were compared using t-tests or Mann-Whitney $U$ tests. Since NPI item scores were not normally distributed, a logistic regression analysis was used to adjust for dementia severity and patient or caregiver age, when significant. For this analysis, NPI symptom and distress scores were dichotomized (score 0 versus $\geq 1$ ). Patient functional impairment (IDDD performance score) was used as a measure of dementia severity.

\section{Results}

\section{Group characteristics}

The caregiver and patient characteristics of both groups are shown in table 1. There were no significant differences between the two groups in caregiver or patient education and sex. However, caregivers and patients in the FTD group were significantly younger than caregivers and patients in the AD group. Furthermore, disease duration was longer, and cognitive functioning and initiative to perform activities were worse in the FTD patients than in the AD patients. There was no difference in actual performance in activities between the groups. 
Table 1. Differences between Alzheimer's disease (AD) and Frontotemporal Dementia (FTD) groups

\begin{tabular}{|c|c|c|c|c|}
\hline & $\begin{array}{l}\text { AD group } \\
(n=47)\end{array}$ & $\begin{array}{l}\text { FTD group } \\
(n=27)\end{array}$ & Test value & P value \\
\hline & Mean (SD) & Mean (SD) & & \\
\hline \multicolumn{5}{|l|}{ Caregiver } \\
\hline Age (years) & $71.3(8.6)$ & $58.5(9.6)$ & $\mathrm{t}=5.9$ & $<0.001$ \\
\hline Education' (1-8) & $3.4(1.9)$ & $3.6(1.6)$ & $t=-0.6$ & 0.575 \\
\hline $\operatorname{Sex}^{2}(m / f)$ & $20 / 27$ & $11 / 16$ & $\chi 2=0.02$ & 0.879 \\
\hline \multicolumn{5}{|l|}{ Patient } \\
\hline Age (years) & $71.5(8.2)$ & $59.5(8.4)$ & $t=6.0$ & $<0.001$ \\
\hline Education' (1-8) & $2.9(2.1)$ & $3.5(1.8)$ & $t=-1.2$ & 0.231 \\
\hline $\operatorname{Sex}^{2}(m / f)$ & $28 / 19$ & $15 / 12$ & $\chi 2=0.1$ & 0.736 \\
\hline \multicolumn{5}{|l|}{ IDDD } \\
\hline Performance & $17.6(10.9)$ & $15.7(13.4)$ & $t=0.7$ & 0.513 \\
\hline Initiative & $24.6(9.3)$ & $16.2(9.9)$ & $t=3.6$ & 0.001 \\
\hline Duration of illness (months) & $39.7(28.5)$ & $60.5(24.3)$ & $t=-3.15$ & 0.002 \\
\hline MMSE-score & $19.3(4.9)$ & $13.2(9.3)$ & $t=3.6$ & 0.001 \\
\hline
\end{tabular}

IDDD = Interview of Daily living activities in Dementia | MMSE - Mini-Mental State examination

1. ranging from primary school (1) to university degree (8) $\mid$ 2. values represent number of males and females

\section{Patient behavioural problems}

Table 2 shows the frequency of behavioural symptoms for the two diagnostic groups. Frequencies refer to the number and proportion of patients in a subgroup showing a specific behavioural symptom. Behavioural symptoms were present in all FTD patients and in $91 \%$ of the AD patients. Apathy was the most common behavioural symptom in both groups, but it occurred more often in the FTD group (89\%) than in the AD group (57\%). In the AD group, anxiety and depression were the next most common symptoms, each being present in more than $50 \%$ of the patients. In the FTD group the other next common symptom was aberrant motor behaviour (82\%), followed by disinhibition (67\%) and irritability (52\%).

Comparison of the mean NPI subscale scores showed that the patients with FTD had significantly higher NPI total scores and higher scores for agitation, euphoria, apathy, disinhibition and aberrant motor behaviour than patients with AD (table 2). Additional logistic regression analyses revealed that after controlling for disease severity (IDDD performance) and patient age, group differences remained significant, except for euphoria (odds ratio $=3.4,95 \% \mathrm{Cl}=0.7-15.4 ; \mathrm{p}=0.113$ ). 
Table 2. Number (\%) of patients with symptoms and mean NPI subscale scores (severity * frequency; range 0-12)

\begin{tabular}{|c|c|c|c|c|c|c|}
\hline \multirow[t]{2}{*}{ NPI subscales } & \multicolumn{2}{|c|}{$\begin{array}{l}\text { Alzheimer Disease } \\
(n=47)\end{array}$} & \multicolumn{2}{|c|}{$\begin{array}{l}\text { Frontotemporal } \\
\text { Dementia } \\
(n=27)\end{array}$} & \multirow[t]{2}{*}{$\begin{array}{l}\text { Test }^{1} \\
\text { Value }\end{array}$} & \multirow[t]{2}{*}{$\begin{array}{l}P^{2} \\
\text { value }\end{array}$} \\
\hline & $\mathrm{N}(\%)$ & Mean (SD) & $N(\%)$ & $\begin{array}{l}\text { Mean } \\
(\mathrm{SD}) \\
\end{array}$ & & \\
\hline Delusions & $10(21.3)$ & $1.1(2.6)$ & $6(22.2)$ & $1.0(2.0)$ & -0.3 & 0.726 \\
\hline Hallucinations & $4(8.5)$ & $0.6(2.1)$ & $4(14.8)$ & $0.5(1.2)$ & -0.7 & 0.492 \\
\hline Agitation & $7(14.9)$ & $0.6(1.5)$ & $13(49.1)$ & $2.4(3.1)$ & -3.2 & 0.001 \\
\hline Depression & 24 (51.1) & $2.4(3.4)$ & $7(25.9)$ & $1.1(2.6)$ & -2.1 & 0.040 \\
\hline Anxiety & $25(53.2)$ & $2.2(3.2)$ & $11(40.7)$ & $2.3(3.7)$ & -0.5 & 0.620 \\
\hline Euphoria & $4(8.5)$ & $0.2(1.0)$ & $12(44.4)$ & $2.0(3.4)$ & -3.7 & $<0.001$ \\
\hline Apathy & $27(57.4)$ & $2.7(3.1)$ & $24(88.9)$ & $5.5(3.5)$ & -3.5 & $<0.001$ \\
\hline Disinhibition & $4(8.5)$ & $0.1(0.4)$ & $18(66.7)$ & $3.1(3.4)$ & -5.7 & $<0.001$ \\
\hline Irritability & $19(40.4)$ & $2.5(3.2)$ & $14(51.9)$ & $5.5(3.8)$ & -1.1 & 0.280 \\
\hline Aberrant motor & $16(34.0)$ & $2.4(3.8)$ & $22(81.5)$ & $5.5(3.8)$ & -3.6 & $<0.001$ \\
\hline Total NPI score & 43 (91.4) & $16.1(17.2)$ & 27 (100\%) & $26.0(16.4)$ & -2.9 & 0.003 \\
\hline
\end{tabular}

NPI = Neuropsychiatric Inventory

1. Mann-Whitney $U$ test | 2. following Bonferonni correction the significance level was set at .0045

\section{Caregiver Distress}

Caregiver distress in relation to individual NPI symptoms was examined in both diagnostic groups (table 3). NPI-D ratings were divided into three categories (Low $=$ score 0 to 1 , Medium = score 2 to 3, and High = score 4 to 5; Kaufer 1998). In the caregivers of AD patients most NPI symptoms (42.9\%) were associated with high distress, 35.7\% with medium distress and $21.4 \%$ with low distress. Apathy, depression and anxiety were most frequently reported as being severely distressing (highest proportion of high distress ratings across total diagnostic group). In the caregivers of FTD patients $30.8 \%$ of the NPI symptoms were associated with high distress, 38.5\% with medium distress and 30.8\% with low distress. The symptoms most frequently reported as being distressing were disinhibition, depression and apathy.

Comparison of both diagnostic groups (Figure 1) showed disinhibition, euphoria and apathy to be significantly more distressing to the caregivers of FTD patients than to the caregivers of AD patients. Additional logistic regression analyses were carried out to test whether these differences in NPI distress subscale scores were associated with disease severity or caregiver age. After controlling for functional impairment (IDDD performance) and caregiver age, group differences remained significant, except for euphoria (odds ratio $=3.9,95 \% \mathrm{Cl}=0.893-17.7 ; \mathrm{p}=0.070$ ). 
Table 3. NPI distress ratings in caregivers of patients with Alzheimer's disease (AD; $n=47$ ) or Frontotemporal Dementia (FTD; $\mathbf{n = 2 7 )}$

\begin{tabular}{|c|c|c|c|c|c|c|c|c|c|c|}
\hline & \multicolumn{2}{|c|}{$n^{1}$} & \multicolumn{2}{|c|}{$\begin{array}{l}\text { No / Low } \\
\text { distress }^{2}\end{array}$} & \multicolumn{2}{|c|}{$\begin{array}{c}\text { Medium } \\
\text { distress }\end{array}$} & \multicolumn{2}{|c|}{$\begin{array}{c}\text { High } \\
\text { distress }\end{array}$} & \multicolumn{2}{|c|}{ Total high $^{3}$} \\
\hline & $A D$ & FTD & $A D$ & FTD & $A D$ & FTD & $A D$ & FTD & $A D$ & FTD \\
\hline \multicolumn{11}{|l|}{ Delusions } \\
\hline $\mathrm{n}$ & 10 & 7 & 2 & 3 & 3 & 1 & 5 & 3 & & \\
\hline$\%$ & & & 20.0 & 2.9 & 30.0 & 14.3 & 50.0 & 42.9 & 10.6 & 11.1 \\
\hline \multicolumn{11}{|l|}{ Hallucinations } \\
\hline$n$ & 4 & 4 & 0 & 3 & 2 & 0 & 2 & 1 & & \\
\hline$\%$ & & & 0 & 75.0 & 50.0 & 0 & 50.0 & 25.0 & 4.3 & 3.7 \\
\hline \multicolumn{11}{|l|}{ Agitation } \\
\hline$n$ & 7 & 13 & 0 & 2 & 2 & 6 & 5 & 5 & & \\
\hline$\%$ & & & 0 & 15.4 & 28.6 & 46.2 & 71.4 & 38.5 & 10.6 & 18.5 \\
\hline \multicolumn{11}{|l|}{ Depression } \\
\hline $\mathrm{n}$ & 24 & 7 & 4 & 0 & 10 & 0 & 11 & 7 & & \\
\hline$\%$ & & & 16.7 & 0 & 41.7 & 0 & 45.8 & 100 & 23.4 & 25.9 \\
\hline \multicolumn{11}{|l|}{ Anxiety } \\
\hline$n$ & 25 & 11 & 4 & 4 & 11 & 4 & 10 & 3 & & \\
\hline$\%$ & & & 16.0 & 36.4 & 44.0 & 36.4 & 40.0 & 27.3 & 21.3 & 11.1 \\
\hline \multicolumn{11}{|l|}{ Euphoria } \\
\hline$n$ & 4 & 12 & 2 & 5 & 2 & 6 & 0 & 1 & & \\
\hline$\%$ & & & 50.0 & 41.7 & 50.0 & 22.2 & 0 & 8.3 & 0 & 3.7 \\
\hline \multicolumn{11}{|l|}{ Apathy } \\
\hline$n$ & 27 & 24 & 7 & 5 & 8 & 13 & 12 & 6 & & \\
\hline$\%$ & & & 25.9 & 20.8 & 29.6 & 54.2 & 44.4 & 25.0 & 25.5 & 22.2 \\
\hline \multicolumn{11}{|l|}{ Disinhibition } \\
\hline $\mathrm{n}$ & 4 & 18 & 3 & 4 & 1 & 6 & 0 & 9 & & \\
\hline$\%$ & & & 75.0 & 22.2 & 25.0 & 33.3 & 0 & 50.0 & 0 & 33.3 \\
\hline \multicolumn{11}{|l|}{ Irritability } \\
\hline $\mathrm{n}$ & 19 & 14 & 6 & 1 & 6 & 11 & 7 & 2 & & \\
\hline$\%$ & & & 31.6 & 7.1 & 31.6 & 8.6 & 36.8 & 14.3 & 14.9 & 7.4 \\
\hline \multicolumn{11}{|l|}{ Aberrant Mot. } \\
\hline$n$ & 16 & 22 & 6 & 13 & 6 & 6 & 4 & 3 & & \\
\hline$\%$ & & & 37.5 & 59.1 & 37.5 & 27.3 & 25.0 & 13.6 & 8.5 & 11.1 \\
\hline \multicolumn{11}{|l|}{ Mean } \\
\hline $\mathrm{n}$ & 14 & 13 & 3 & 4 & 5 & 5 & 6 & 4 & & \\
\hline$\%$ & & & 21.4 & 30.8 & 35.7 & 38.5 & 42.9 & 30.8 & 11.9 & 14.8 \\
\hline
\end{tabular}

1. $n=$ number of caregivers whose spouse (patient) exhibited the symptom | 2. distress rates (\%) per diagnostic group when the patient exhibits the symptom | 3. percentage of caregivers reporting high level of distress within total diagnostic subgroup (AD/FTD)

In addition, the general caregiver burden and caregiver competence were compared between the caregivers of AD and FTD patients using regression analysis controlled for disease severity and caregiver age. The caregivers of FTD patients experienced caregiving 
as being more of a burden ( $t=3.4, p=0.001)$. There was no differences in total caregiver competence scores ( $t=-1.5, p=0.124)$, but when examining the three subscales of the sense of competence scale, caregivers of FTD patients felt less satisfied with the patient (t $=-2.9, p=0.005)$ and with themselves as caregiver $(t=-2.1, p=0.037)$. However, caregivers of $A D$ patients experienced more consequences for their personal life $(t=3.1, p$ $=0.003)$.

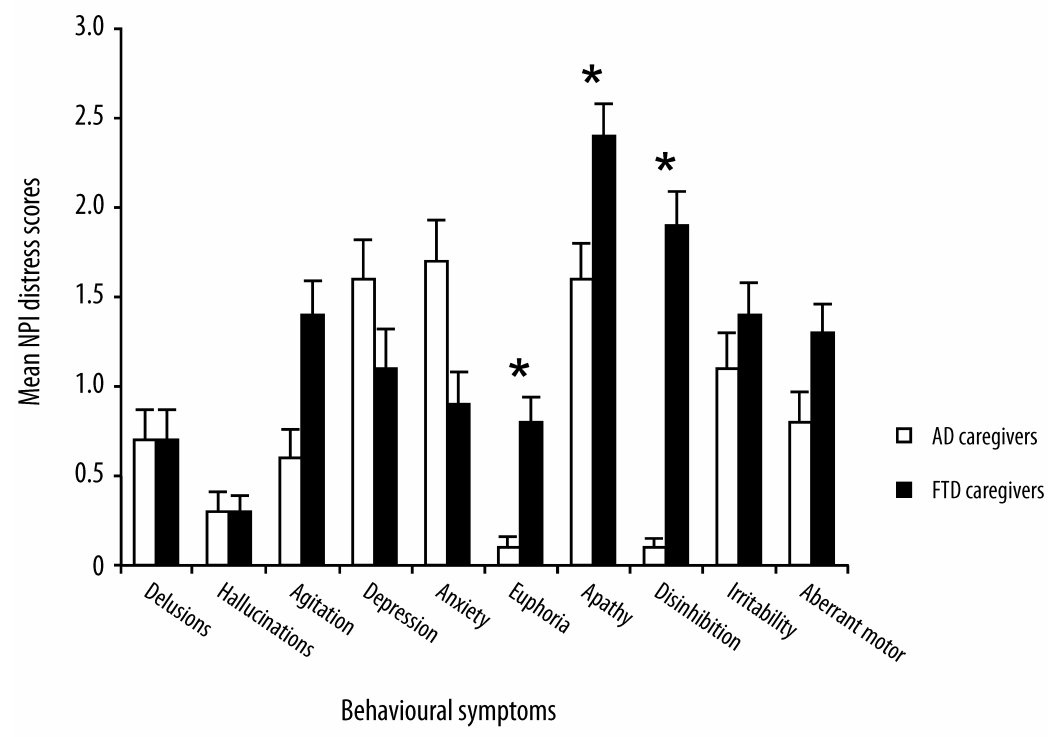

Figure 1. Mean distress $( \pm$ SEM) ratings for NPI symptoms in caregivers of patients with FrontoTemporal dementia (FTD) and patients with Alzheimer's disease (AD) $\mid * P<0.05$

\section{Discussion}

Although many studies have investigated the behavioural changes in FTD, none have investigated these behavioural problems in relation to caregiver distress (Bathgate et al. 2001; Bozeat et al. 2000; Diehl and Kurz 2002; Levy et al. 1996; Rymer et al. 2002; Snowden et al. 2001). We found that the behavioural symptom profile was different in patients with FTD and AD, and that differences existed between both diagnostic groups in the level of caregiver distress related to these symptoms. 
Patients with FTD had significantly higher levels of agitation, apathy, disinhibition and aberrant motor behaviour than patients with $A D$ when the level of functional impairment was taken into account. The observed differences are largely consistent with the results of Levy et al. (1996) who also used the NPI to assess behavioural symptoms. However, Levy et al. found that FTD patients exhibited more euphoria, but they found no differences in agitation. The difference in findings between the two studies may be due to differences in dementia severity between groups and different operationalisations of dementia severity (cognitive impairment in the study of Levy et al. versus functional impairment in the current study). We did find higher levels of euphoria in FTD patients when functional impairment was not taken into account.

To our knowledge, this study is one of the first to examine distress related to patient behavioural problems experienced by caregivers of patients with FTD or AD. The caregivers of patients with FTD experienced patient disinhibition and apathy as highly distressing. Moreover, although not many patients were depressed (25\%), their caregivers experienced patient depression as being highly distressing. Aberrant motor behaviour, which affected $81 \%$ of the FTD patients, was most often rated as not or slightly distressing. These findings support previous findings that some problem behaviours have a greater impact on caregivers than others (Kaufer 1998; Robinson et al. 2001; Victoroff et al. 1998), which underscores the importance of focusing on specific behavioural changes when studying and trying to ameliorate caregiver distress.

Comparison of the caregivers of FTD patients and the caregivers of AD patients revealed that when behavioural problems were present most symptoms (42.9\%) were associated with high distress in the caregivers of AD patients, whereas most symptoms (38.5\%) were associated with medium distress in the caregivers of patients with FTD. But, caregivers of FTD patients had higher mean distress score than caregivers of FTD patients. Disinhibition and apathy in particular had higher mean distress scores in the FTD group compared to the $A D$ group. However, again in the AD group caregivers most frequently reported apathy as highly distressing when the symptom was present, while in the FTD group caregivers most frequently reported apathy as medium distressing when present. Therefore, higher mean distress levels in the FTD group are probably caused by a higher level of behavioural problems in FTD patients. The caregivers of FTD patients also reported higher levels of general caregiver burden than did the caregivers of AD patients, even after controlling for dementia severity and caregiver age. Caregivers of FTD patients 
also felt less satisfied with the patient and themselves as caregiver. On the other hand, AD caregivers experienced more consequences for their personal life, for example a lack of privacy. An explanation for this finding may be that AD patients need more continuous supervision than FTD patients because of their cognitive impairment, which requires greater interference of the caregiver and consequently less time for themselves compared to FTD caregivers.

The higher levels of distress experienced by the caregivers of FTD patients are not surprising given the fact that early-onset dementia is often not correctly diagnosed at an early stage, which results in frustration for patient and caregiver. In addition, early-onset dementia gives additional problems because it appears when most people are enjoying an active and independent lifestyle. Behavioural changes are a more salient characteristic of FTD than of AD (Diehl and Kurz 2002) and are a well-known risk factor for caregiver stress (Donaldson et al. 1997; Robinson et al. 2001; Victoroff et al. 1998). Problems related to disinhibition are probably socially embarrassing to caregivers and hamper pleasant social interactions, causing the caregiver greater distress (Pruchno and Resch 1989; Rymer et al. 2002). Apathy was also stressful to the caregivers of FTD patients, as is was in other studies of patients with dementia (Greene et al. 1982; Landes et al. 2001; LoGiudice et al. 1995; Murray et al. 1999; Vugt et al. 2003). Apathy diminishes the interaction between patient and caregiver, harming their relationship (Vugt et al. 2003). Finally, differences in the level of stress may have been influenced by differences in availability of support between the groups. Support services are mostly established for the geriatric population and therefore the older $\mathrm{AD}$ patients and caregivers may have better access to these services than the younger FTD group. In addition, there may have been differences in family support, because FTD patients had probably younger children than AD patients.

The study has some potential limitations. Firstly, the risk off circularity exists when selecting FTD patients on the basis of behavioural changes in order to compare the problem behaviours of patients with AD and FTD. We minimized this by diagnosing patients on the basis of neurological, neuropsychological and neuro-imaging data and by selecting patients on the basis of well defined diagnostic criteria (The Lund and Manchester (groups 1994). Secondly, the issue of matching AD and FTD patients should be discussed. To compare specific aspects of behaviour in FTD patients with AD patients, one must ensure the overall comparability of the two groups. However, differences in disease course and symptomatology between AD and FTD might render traditional measures of dementia severity invalid (Brandt and Munro 2002). Using the MMSE as a 
measure of dementia severity in this study is problematic because of the influence of lack of motivation or cooperation, perseveration and language problems on the test score of FTD patients (Brandt and Munro 2002). Likewise, disease duration was considered unsuitable because of differences in disease course between AD and FTD. Therefore, we used an alternative strategy by adjusting for functional impairment in the analysis. Thus, it is not likely that group differences in this study are due to differences in dementia severity. Thirdly, group comparisons were adjusted for caregiver age in addition to dementia severity as potential confounders of group differences in caregiver distress. But, the way in which a caregiver reacts to a patient's behaviour is probably determined by several factors, for example caregiver coping styles, social support and quality of the relationship. Future studies into caregiver distress should explore the impact of these factors.

Our findings underscore the importance of distinguishing between diagnostic groups when investigating caregiver reactions to problem behaviour. They may also facilitate the development of more specific caregiver interventions. For example, the caregivers of FTD patients found excessive and apathetic behaviour to be particularly distressing. However, most caregiver training programs mainly target patient behavioural excesses and do not focus on apathy. Our results suggest that such programmes should focus on problems related to disinhibition as well as apathy in patients with FTD in order to reduce caregiver distress.

\section{Key points}

- $\quad$ FTD caregivers experience high distress levels due to patient apathy, depression, and disinhibition

- $\quad A D$ caregivers experience high distress levels due to patient apathy, depression, and anxiety

- $\quad$ FTD caregivers report higher distress levels due to disinhibition and apathy than AD caregivers

- $\quad$ FTD caregivers experience more burden en feel less competent than $\mathrm{AD}$ caregivers 


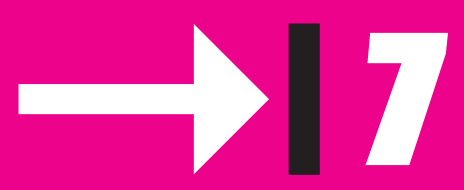

The effects of behavioural symptoms on institutionalisation in dementia 


\section{Abstract}

Objectives: Literature on the effects of problem behaviours in dementia on the decision to institutionalise the patient has shown conflicting results. Few studies took into account that specific behavioural problems may have differential effects on the decision to institutionalise the patient. Moreover, it is probably not patient behaviour itself that causes nursing home placement (NHP), but caregiver's emotional reaction to it. The aim of the present study was to examine the impact of specific behavioural sub-syndromes and caregiver's emotional reaction on NHP.

Methods: Hundred-nineteen patients with dementia and their informal caregivers were followed up for 2 years. Time to NHP was measured in months from the date of the baseline interview to the date of NHP. Behavioural disturbances in the patient and caregivers' emotional reactions were measured with the NeuroPsychiatric Inventory (NPI). A Cox regression analysis was used to analyze the probability that caregivers would institutionalize the patient sooner when patient behavioural problems or caregiver distress were present at baseline.

Results: Results of the present study showed that $41 \%$ of the patients were institutionalised during the two year follow-up. Caregiver distress related to patient behaviour was a significant predictor of NHP, while behaviour in itself did not predict NHP. Contrary to our expectations, we did not find a differential impact of specific aspects of problem behaviour. Furthermore, children caregivers, especially daughters, were associated with shorter time to NHP compared to spouses.

Conclusions: Findings indicate that caregiver's emotional reaction to patient behaviour is more critical than problem behaviours per se in the decision to institutionalise the patient. Interventions aimed at teaching caregivers strategies to better manage difficult patient behaviours may provide caregivers the necessary resources to continue care at home. Future interventions need to account for the specific needs and problems of different caregiver groups. 


\section{Introduction}

Two-third of the patients with dementia are cared for at home by relatives. Caring for a dementia patient is a stressful and demanding process that affects caregivers' psychological and physical well-being (Pinquart and Sorensen 2003; Schulz et al. 1995). In particular, the behavioural and psychological symptoms in dementia (BPSD) such as depression, apathy, agitation and aggressiveness are known to be more stressful to caregivers than the cognitive or functional problems in the patient (Donaldson et al. 1998; Hooker et al. 2002; Vugt et al. 2003). Patient behavioural problems have been identified as particularly important in the decision to institutionalise the patient (Chan et al. 2003; O'Donell et al. 1992; Yaffe et al. 2002).

Several studies into BPSD as a predictor of nursing home placement (NHP), yielded mixed results so far. The majority of these studies found an association between BPSD and NHP (Banerjee et al. 2003; Chan et al. 2003; Cohen et al. 1993; Gaugler et al. 2000b; Gaugler et al. 2003; Hope et al. 1998; Kim et al. 2002; Knopman et al. 1988; Lopez et al. 1999; Phillips and Diwan 2003; Yaffe et al. 2002), while others did not (Fisher and Lieberman 1999; Hebert et al. 2001; Knopman et al. 1999; Lieberman and Kramer 1991; Spruytte et al. 2001). Contradictory findings in past research may be due to the following. First, different indicators of patient behaviour were used to measure BPSD. However, specific aspects of BPSD vary in there impact on caregivers (Victoroff et al. 1998; Vugt et al. 2003). Despite the extensive literature on predictors of NHP, only a handful of studies addressed specific aspects of BPSD (Chan et al. 2003; Hope et al. 1998; Knopman et al. 1988; Lopez et al. 1999). Knopman and colleagues selected nocturnal disruptive behaviour and excessive irritability for their analyses and found that it increased the risk of institutionalisation. Hope and colleagues included several symptoms in the analyses (e.g. anxiety, hallucinations, persecutory ideas, sadness, activity disturbances, abnormal eating, and night-time activity), and found only night-time activity to predict NHP. In a study of Chan and colleagues it was found that symptoms of agitation were significantly associated with shorter time to NHP in an univariate analysis, whereas patient depression or psychosis were not. However, they did not test these specific aspects of BPSD in a multivariate analysis. Lopez and colleagues included patient psychosis, insomnia, wandering, aggression, agitation and depression in their model and found only psychosis to be related with institutionalisation. Second, none of the reported studies did investigate the importance of caregiver's emotional reaction to BPSD. However, some patient behavioural problems may be troublesome for some caregivers but not for others. In other words, one 
might hypothesise that the decision to institutionalise does not so much depend on patient behaviour itself, but more on caregiver's emotional reaction to BPSD. Furthermore, the context of care has an impact on perceptions of care demands and stress (Gaugler et al. 2000a). Therefore caregiver characteristics, such as kinship and gender, as well as patient characteristics, such as disease severity, must be taken into account when examining predictors of NHP.

The present study will extend the findings of previous research by focussing on the role of specific aspects of BPSD, and caregiver's emotional reaction to BPSD in predicting NHP. We did this in a 2-year prospective design, adjusted for patient as well as caregiver characteristics. The first aim of the present study is to examine the relationship between specific aspects of BPSD and patient NHP. The second aim is to investigate the role of caregiver distress in the relationship between patient behavioural problems and NHP.

\section{Methods}

\section{Subjects}

Subjects were 119 informal caregivers of ambulatory patients with dementia according to DSM-IV (American Psychiatric Association, 1994). The present study is part of the Maastricht Study of Behaviour in Dementia (abbreviated as MAASBED). MAASBED is a 2year follow-up study that focuses on the course and risk factors of behavioural and psychological symptoms of dementia (BPSD). Patients and their caregivers are seen at 6month intervals. Patients were referred by the Memory Clinic of the Academic Hospital Maastricht, or the geriatric division of the Regional Institute for Community Mental Health (RIAGG), Maastricht, the Netherlands. Data on clinical symptoms, neuropsychological testing, laboratory studies and neuro-imaging were discussed in plenary clinical rounds to diagnose patients according to DSM-IV criteria.

Caregivers were included if they were the primary caregiver and had contact with the patient at least once a week. All patients were living at home at baseline. Informed consent was obtained from all subjects. Patient and caregiver assessments were conducted by independent, clinically experienced, trained psychologists.

In the current study 1-year follow up data were available for 97 (81.5\%) patients and caregivers, and 2-year follow up data were available for 75 (63.0\%) patients and caregivers. Missing values in the follow up were due to refusal $(n=18 ; 15.1 \%)$ or death of the patient $(n=26 ; 21.8 \%)$. Caregivers lost to follow-up were relatively older $(t=-2.8, p=0.006)$, patients were more cognitively impaired on the MMSE $(t=2.1, p=0.034)$ and dementia 
was more severe on the Global Deterioration Scale $(t=-2.0, p=0.045)$. There were no other differences in patient or caregiver characteristics at baseline between the follow up sample and the loss to follow-up group.

Sample characteristics are shown in table 1. Ninety patients had Alzheimer's disease, 20 vascular dementia, 2 Frontotemporal dementia, 3 Parkinson's disease, 1 primary progressive dementia, and 3 mixed dementia (AD/vascular).

\section{Dependent variable}

The dependent variable was time to NHP, assessed by interviews with the caregiver at baseline, 6, 12, 18, and 24 months. It was measured in months from the date of the baseline interview to the date of NHP.

\section{Patient measures}

Patient behavioural problems were measured with the NeuroPsychiatric Inventory (NPI; Cummings 1994), a structured interview with the caregiver that evaluates 12 neuropsychiatric symptoms. The total score on each item can range from 1 to 12 and is obtained by multiplying severity ( 1 "mild" to 3 "severe") by frequency (1 "sometimes" to 4 "very often"). Principal component analysis was used to identify behavioural subsyndromes measured by the NPI. This resulted in three components, explaining 55\% of variance in the data (Aalten et al. 2003): (1) a hyperactivity factor, including the symptoms disinhibition, irritability, agitation, euphoria, and aberrant motor behaviour (5 items, Cronbach's alpha $=0.73$ ); (2) a mood/apathy factor including depression, apathy, nighttime behavioural disturbances and eating abnormalities ( 4 items, $\alpha=0.63$ ); and (3) a psychosis factor, including hallucinations and delusions ( 2 items, $\alpha=0.72$ ). The symptom anxiety loaded high on several factors and therefore was not included in the analysis. Total scores for each sub syndrome were computed as the sum of observed NPI item scores for each factor.

The Mini Mental Status Examination (MMSE; Folstein et al. 1975) was used to measure the patient cognitive functioning of the patients. The dependence on others of the patient with regard to daily activities was measured with the Interview for Deterioration in Daily living activities in Dementia (IDDD; Teunisse 1995). This questionnaire consists of 20 items reflecting initiative to and actual performance of daily activities. We used a summed score for items concerning actual performance of activities (range 0 to 44) and for items 
concerning initiative to activities (range 0 to 36). The severity of dementia was rated with the Global Deterioration Scale (GDS; Reisberg et al. 1982).

\section{Caregiver measures}

For each of the 12 behavioural symptoms on the NPI, caregivers rated the level of distress they experienced on a scale from 0 (none) to 5 (extreme). NPI-Distress score is the sum of these 12 ratings (range $0-60$ ).

Caregiver's subjective competence was measured with the Short Sense of Competence Questionnaire (SOC; Vernooij-Dassen et al. 1999). This questionnaire consists of 7 items rated on a 5-point scale (1 "agree very strongly" to 5 "disagree very strongly"; range 7-35). These items reflect three domains of caregivers' feelings of being capable of caring for a demented person: (a) satisfaction with the demented person as a recipient of care; (b) satisfaction with one's own performance as a caregiver; and (c) consequences of involvement in care for the personal life of the caregiver. The content validity and construct validity have been reported elsewhere (Vernooij-Dassen et al. 1999). Cronbach's alpha in this study is 0.74 .

Depressive symptoms were measured with the Montgomery-Asberg Depression Rating Scale (MADRS; Montgomery and Asberg 1979), a structured interview administered by the clinician. Ratings (from 0 to 6) on 10 items were summed (range 0-60).

\section{Data analysis}

Groups were compared at baseline in patient and caregiver characteristics using t-tests and Chi-square tests. Cox regression was used to analyse the probability that caregivers would institutionalise the patient during the 2-year follow up when patient behavioural problems were present at baseline. Months to NHP was the dependent variable. Baseline measurements of BPSD total score, mood/apathy, psychosis, and hyperactivity were the predictors. BPSD scores were dichotomized with a median split; i.e. high BPSD total score $\geq 18$, high mood/apathy $\geq 6$, high hyperactivity $\geq 3$, high psychosis $\geq 1$. First, the relationship between total BPSD and NHP was tested in a hierarchical Cox regression model to specify the unique contribution of patient background variables (step 1: disease severity, medication use), caregiver demographics (step 2: age, sex, education, kinship) and patient behaviour (step 3). A second analysis was performed with patient mood/apathy, psychosis, and hyperactivity entered as predictors in the model at step 3. In addition, the same two analyses were performed with caregiver distress related to the 
behavioural problems (total distress; mood/apathy distress, psychosis distress, and hyperactivity distress) as predictor of NHP.

Distress scores were dichotomized with a median split; i.e. high total distress score $\geq 10$, high mood/apathy distress score $\geq 4$, high hyperactivity distress score $\geq 2$, high psychosis distress score $\geq 1$. Significance level was set at $\alpha=.050$. All tests were performed twotailed.

\section{Results}

\section{Nursing home placement and baseline group differences}

Of the 119 patients 16 (13.4\%) patients were institutionalised during the first 6 months, 12 (10.1\%) patients between 6 and 12 months, 13 (10.9\%) patients between 12 and 18 months, and 8 (6.7\%) patients between 18 and 24 months. Overall, 49 (41.1\%) of the dementia patients were institutionalised over the 2 year follow up.

Table 1 shows baseline differences between institutionalised patients and patients living at home during the 2 year follow-up. There were no differences between both groups in patient behavioural problems, disease severity, cognitive status or functional impairment (all $p$ values were higher than 0.053). Institutionalised patients were more often woman and significantly older than patients living at home. There were no differences between caregivers of patients living at home and the institutionalised group in education, gender, or length of caregiving (all p values were higher than 0.276). However, caregivers of institutionalised patients were significantly younger, more often children, had less contact hours with the patient, reported significantly higher levels of emotional distress and depressive feelings, and felt less competent compared to the caregivers of patients living at home.

\section{Patient behaviour as a predictor of NHP}

Hierarchical Cox regression analysis was used to analyse the time to NHP for patients with high versus low levels of BPSD at baseline. Results indicated that the total model including patient background characteristics (step 1), caregiver demographics (step 2) and total BPSD (step 3) predicted NHP $\left(\chi^{2}=18.4, d f=7, p=0.010\right)$. Block 1 showed no significant effect of patient variables $\left(\chi^{2}=3.9, d f=2, p=0.138\right.$ ). Block 2 , on the other hand, showed a 
significant change with the addition of caregiver demographics in the model $\left(\chi^{2}=14.1, \mathrm{df}\right.$ $=4, p=0.007$ ). However, block 3 showed no significant change with the inclusion of patient behaviour in the model $\left(\chi^{2}=0.5, d f=1, p=0.495\right)$, indicating that patient behaviour was not a significant predictor of $\operatorname{NHP}(\operatorname{Exp}(B)=1.2, p=0.497)$. Kinship was the only significant predictor in the total model $(\operatorname{Exp}(B)=3.1, p=0.012)$, with children and other relatives institutionalising the patient sooner than spouses.

\section{Table 1. Patient and caregiver differences}

\begin{tabular}{|c|c|c|c|c|c|}
\hline & $\begin{array}{l}\text { Total } \\
\mathrm{n}=119\end{array}$ & $\begin{array}{l}\text { Institutionalized } \\
\mathrm{n}=49\end{array}$ & $\begin{array}{l}\text { Home } \\
n=70\end{array}$ & Test value & P value \\
\hline & Mean (SD) & Mean (SD) & Mean (SD) & & \\
\hline \multicolumn{6}{|l|}{ Patient } \\
\hline Age & $78.5(8.4)$ & $80.8(7.1)$ & $76.8(8.8)$ & $t=-2.6$ & 0.011 \\
\hline \multicolumn{6}{|l|}{$\operatorname{Sex}(n, \%)$} \\
\hline $\begin{array}{r}\text { Male } \\
\text { Female }\end{array}$ & $\begin{array}{l}49(41.2 \%) \\
70(58.8 \%)\end{array}$ & $\begin{array}{l}14(28.6 \%) \\
35(71.4 \%)\end{array}$ & $\begin{array}{l}35(50 \%) \\
35(50 \%)\end{array}$ & $\chi^{2}=5.5$ & 0.019 \\
\hline GDS & $4.1(0.7)$ & $4.2(0.6)$ & $4.0(0.8)$ & $\mathrm{t}=-1.1$ & 0.278 \\
\hline Illness duration (months, SD) & $42.9(31.0)$ & $38.8(29.9)$ & $49.6(31.0)$ & $t=0.2$ & 0.862 \\
\hline MMSE & $18.3(4.7)$ & $17.6(4.2)$ & $18.8(4.9)$ & $t=1.4$ & 0.170 \\
\hline NPI total score & $21.8(21.9)$ & $24.9(22.6)$ & $19.6(21.4)$ & $t=-1.9$ & 0.058 \\
\hline \multicolumn{6}{|l|}{ IDDD } \\
\hline $\begin{array}{r}\text { Initiative } \\
\text { Performance }\end{array}$ & $\begin{array}{l}22.5(9.6) \\
20.1(10.9)\end{array}$ & $\begin{array}{l}20.4(9.3) \\
20.7(10.2)\end{array}$ & $\begin{array}{l}23.9(9.6) \\
19.7(11.5)\end{array}$ & $\begin{array}{l}t=1.9 \\
t=-0.5\end{array}$ & $\begin{array}{l}0.053 \\
0.619 \\
\end{array}$ \\
\hline \multicolumn{6}{|l|}{ Caregiver } \\
\hline $\begin{array}{l}\text { Sex }(n, \%) \\
\text { Age }\end{array}$ & $\begin{array}{l}42(35.3 \%) \\
77(64.7 \%) \\
63.8(12.2)\end{array}$ & $\begin{array}{l}19(38.8 \%) \\
30(61.2 \%) \\
60.1(11.9)\end{array}$ & $\begin{array}{l}23(32.9 \%) \\
47(67.1 \%) \\
66.5(11.7)\end{array}$ & $\begin{array}{l}\chi^{2}=0.4 \\
t=2.9\end{array}$ & $\begin{array}{l}0.506 \\
0.005\end{array}$ \\
\hline $\begin{array}{l}\text { Relationship (n, \%) } \\
\text { Education level' (n, \%) }\end{array}$ & $\begin{array}{l}64(53.8 \%) \\
47(39.5 \%) \\
8(6.7 \%)\end{array}$ & $\begin{array}{l}17(34.7 \%) \\
28(57.1 \%) \\
4(8.2 \%)\end{array}$ & $\begin{array}{l}47(67.1 \%) \\
19(27.1 \%) \\
4(5.7 \%)\end{array}$ & $\chi^{2}=12.5$ & 0.002 \\
\hline $\begin{array}{r}\text { Low } \\
\text { Medium } \\
\text { High }\end{array}$ & $\begin{array}{l}41(34.5 \%) \\
52(43.7 \%) \\
26(21.8 \%)\end{array}$ & $\begin{array}{l}14(28.6 \%) \\
21(42.9 \%) \\
14(28.6 \%)\end{array}$ & $\begin{array}{l}27(38.6 \%) \\
31(44.3 \%) \\
12(17.1 \%)\end{array}$ & $\chi^{2}=2.6$ & 0.276 \\
\hline $\begin{array}{r}\text { Contact per week }(\mathrm{n}, \%) \\
\text { Low } \\
\text { High }\end{array}$ & $\begin{array}{l}47(39.5 \%) \\
72(60.5 \%)\end{array}$ & $\begin{array}{l}27(55.1 \%) \\
22(44.9 \%)\end{array}$ & $\begin{array}{l}20(28.6 \%) \\
50(71.4 \%)\end{array}$ & $\chi^{2}=8.5$ & 0.004 \\
\hline Duration of care (months, SD) & $27.9(25.5)$ & $26.8(25.4)$ & $28.8(25.8)$ & $t=0.4$ & 0.677 \\
\hline NPI distress & $11.1(9.9)$ & $13.6(10.4)$ & $9.3(9.2)$ & $t=-2.7$ & 0.007 \\
\hline Sense of Competence & $23.7(5.9)$ & $22.0(5.9)$ & $24.9(5.6)$ & $\mathrm{t}=2.7$ & 0.009 \\
\hline MADRS & $8.3(6.2)$ & $9.8(7.0)$ & $7.3(5.3)$ & $\mathrm{t}=-2.0$ & 0.046 \\
\hline
\end{tabular}

GDS = Global Deterioration Scale $\mid$ MMSE = Mini Mental State Examination | NPI = Neuropsychiatric Inventory | IDDD $=$ Interview of Daily living activities in Dementia | MADRS = Montgomery Asberg Rating Scale

1. educational level was compressed from eight to three levels: low (level 1 and 2), medium (level 3 to 5), and high (level 6 to 8) 
To examine the differential impact of specific behavioural sub-syndromes, the same hierarchical Cox regression analyses was performed with patient hyperactivity, psychosis and mood/apathy entered in the model at step 3. Results showed that patient hyperactivity, mood/apathy and psychosis were no significant predictors of NHP (respectively $\operatorname{Exp}(B)=1.2, p=0.590 ; \operatorname{Exp}(B)=1.1, p=0.711 ; \operatorname{Exp}(B)=1.3, p=0.432$ ).

\section{Caregiver distress as a predictor of NHP}

Next, hierarchical Cox regression analysis was performed to examine the impact of caregiver distress related to BPSD on time to NHP. First, the model with patient characteristics (step 1), caregiver demographics (step 2) and total distress (step 3) was tested. Results indicate that the total model predicted $\mathrm{NHP}\left(\chi^{2}=24.7, \mathrm{df}=7, \mathrm{p}=0.001\right)$, with a significant change at step 2 , and also at step 3 when caregiver distress was entered in the model $\left(\chi^{2}=6.6, d f=1, p=0.010\right)$. In the total model kinship $(\operatorname{Exp}(B)=3.1, p=0.012)$ as well as caregiver distress were significant predictors of $\mathrm{NHP}(\operatorname{Exp}(B)=2.7, p=0.023)$, with children and high distressed caregivers institutionalising the patient sooner. We explored the relationship between BPSD related distress and other psychological characteristics of the caregiver with Pearson correlations. Results indicated that distress was significantly correlated with feelings of competence ( $r=-.47, p \leq 0.001)$ and depressive symptoms in the caregiver $(r=.28, p \leq 0.002)$.

Next, this hierarchical Cox regression analysis was performed with distress related to specific behavioural sub-syndromes. Results showed that distress related to patient hyperactivity, mood/apathy and psychosis were no significant predictors (respectively $\operatorname{Exp}(B)=1.7, p=0.150 ; \operatorname{Exp}(B)=0.9, p=0.677 ; \operatorname{Exp}(B)=1.5, p=0.217)$. Kinship was the only significant predictor in the total model $(\operatorname{Exp}(B)=2.9, p=0.019)$.

\section{Post-hoc analysis of kinship impact}

The impact of kinship was further explored in a post-hoc analysis, because the relationship with the patient showed to be an important predictor of NHP. Analysis of differences in NHP between more specific relationship categories (i.e., wives, husbands, daughters, and sons) indicated that caregivers of institutionalised patients were more often daughters, while wives were least likely to institutionalise the patient $\left(\chi^{2}=14.6 ; p=0.002\right)$. Furthermore, exploration of differences in other characteristics of spouses and children showed differences in competence $(t=2.6, p=0.010)$ and distress $(t=2.5, p=0.012)$, with daughters feeling most distressed and least competent. There were no differences between the groups in depressive symptoms ( $t=0.7, p=0.459)$. 


\section{Discussion}

Results of the present study showed that $41 \%$ of the patients were institutionalised during the two year follow-up. Caregiver distress related to BPSD was a significant predictor of NHP, while BPSD in itself did not predict NHP. Also children caregivers, especially daughters, were associated with shorter time to NHP compared to spouses.

The finding that BPSD was no significant predictor of NHP in multivariate analyses is consistent with several other studies (Chan et al. 2003; Hebert et al. 2001; Pruchno et al. 1990). We hypothesised that caregiver's emotional reaction to BPSD may be a more important risk factor of NHP than patient behaviour itself. The results of the present study supports this hypothesis, and is also in line with several studies that found caregiver burden to be predictive for NHP (Cohen et al. 1993; Gaugler et al. 2003; Hebert et al. 2001; Lieberman and Kramer 1991; Yaffe et al. 2002). Caregivers apparently differ in their emotional responses to BPSD even when facing similar problems. These results indicate that caregiver's perception of patient problems is more critical than problem behaviour per se in the decision to institutionalise the patient. So, the focus should be on caregiver distress rather than on presence of problem behaviours when addressing the risk for NHP. Contrary to our expectations, we did not find a differential impact of distress related to specific aspects of BPSD. This indicates that the decision to institutionalise depends on the level of caregiver distress per se, and is not related to specific aspects of patient behaviour. Although, some aspects of behaviour may be more stressful to caregivers than others, differentiation in distress related to specific aspects of BPSD does not necessarily discriminate between distressed and non-distressed caregivers. Several behavioural problems may arise in the same patient and induce caregiver stress. Caregivers who experience distress due to hyperactivity symptoms in the patient may also report distress related to psychosis. Also the contrary may occur, that caregivers who report low levels of distress related to mood/apathy may report high levels of distress related to hyperactivity. Caregiver distress related to total BPSD does discriminate between high and low distressed caregivers and therefore may be a better predictor of NHP.

The only significant caregiver demographic characteristic as predictor of NHP was kinship. Children, especially daughters, were most likely to institutionalise the patient sooner. This is in line with a study of Hope and colleagues (1998) who found that spouses had a high commitment to care, whereas younger caregivers (often children) were less committed. They probably have other competing life responsibilities such as jobs or children to care for. In the current study gender did not predict the decision to institutionalise, but 
daughters were more likely to institutionalise the patient than sons. This might be explained by a previous finding from the MAASBED study on differences in caregiver strategies (Vugt et al. 2004). Male caregivers tend to use a "supporting care strategy" and only intervene with the patient when needed. Female caregivers are more likely to use a "nurturing care strategy" and feel responsible for all household and personal care activities. This strategy may more easily result in feelings of overload or exhaustion. Indeed, the results of the current study indicated that daughters felt most distressed and least competent compared to other caregivers.

There were several limitations to this study. First, institutionalisation may not always immediately follow the decision to give up home care. For example, lack of available nursing home beds may probably have extended care at home, while physical health problems in the patient may have necessitated earlier institutionalisation. In addition, waiting lists and care indication may differ between different regions. However, this can not explain the kinship differences as it probably concerns a non-selective bias. Second, other aspects of patient functioning than BPSD and dementia severity, such as cognitive and functional status, were not included in the study as predictors of NHP. Patient cognitive and functional status may have to some extent a predictive value with respect to NHP (Severson et al. 1994), although the lack of significant baseline differences makes it not very likely that these variables could explain our results. Finally, the follow-up interval in the present study is confined to 2 years. Longitudinal follow-up until death or institutionalisation of all patients would be needed to obtain a more complete picture of NHP predictors in all stages of dementia.

In conclusion, the major finding of our study is that not presence of BPSD on itself, but caregiver's emotional reaction to patient problems shortened time to NHP. Furthermore, daughters were more likely to institutionalise the patient sooner than spouses. These findings indicate that interventions aimed at teaching caregivers strategies to better manage difficult patient behaviours may perhaps be more effective to extent care at home, than interventions that aim at the reduction of BPSD alone. Finally, future interventions need to account for the unique needs and stresses of specific groups of family caregivers. 


\section{Key points}

- $\quad 41 \%$ was institutionalised during 2-year follow up

- Caregiver distress due to patient behaviour, but not behaviour in itself, is a risk factor for patient institutionalisation

- Daughter caregivers institutionalise the patient sooner than spouses 


\section{$\rightarrow \mid 8$ \\ Influence of caregiver management strategies on patient behaviour in dementia}




\section{Abstract}

Objectives: Little is known about the effectiveness of caregiver management strategies on the functioning of the demented patient. However, identification of specific caregiver strategies may provide useful information on the management and manifestation of behavioural problems in dementia.

Methods: Ninety-nine patients with dementia and their informal caregivers were followed up for 1 year. Interviews were used to assess differences in caregiver management strategies. Behavioural disturbances in the patient were measured with the NeuroPsychiatric Inventory (NPI). Repeated measures analyses were carried out to investigate the relationship between caregiver management strategies and patient behaviour.

Results: Three caregiver management strategies were identified, based on whether caregivers accepted, or not, the caregiving situation and dementia related problems. Caregivers characterized by non-acceptance were typified as 'Non-adapters'; caregivers characterized by acceptance were further subdivided into two groups typified as 'Nurturers' and 'Supporters'. Caregiver characteristics such as sex, education and personality were important determinants of management strategies. ANCOVA showed that non-adapters reported significantly more hyperactivity symptoms in patients and felt less competent than did supporters.

Conclusions: Caregiver management strategies would appear to be associated with behavioural problems in dementia, and are important in predicting patient behaviour and caregiver burden. Intervention programmes should aim at teaching caregivers adequate management strategies. 


\section{Introduction}

Two-third of the patients with dementia is cared for at home by relatives. Research has shown that particularly behavioural and psychological symptoms in dementia (BPSD) are associated with negative caregiver consequences - they contribute to caregiver burden (Donaldson et al. 1998; Draper et al. 1995), have a negative impact on the relationship between caregiver and patient ((Lawrence et al. 1998; Vugt et al. 2003), and increase the risk of institutionalisation (O'Donell et al. 1992). The quality of life of both patient and caregiver, and the ability to provide care at home are greatly dependent on the ability of the caregiver to adequately adapt and respond to these behavioural problems and to the changing needs of the patient. However, caregivers differ in their management strategies, with some caregivers being more successful than others. Insight into what constitutes a successful strategy is lacking.

Previous research has focused mainly on general coping strategies in caregivers dealing with dementia related problems. Few studies have specifically looked at daily care management strategies in dementia. Hinrichsen and Niederehe (1994) identified three types of dementia management strategies, namely, criticism, encouragement, and active management. The use of active management and criticism was related to a greater caregiver burden, whereas encouragement was associated with less of a caregiver burden and less desire to institutionalise the patient.

Differences between individuals regarding management strategies could be ascribed to the context in which management takes place (e.g. disease status and patient characteristics) as well as to caregiver characteristics. Some studies have found sex differences in dementia management strategies. For example, Corcoran (1992) suggested that male caregivers have a more task-oriented approach and carry out activities in a linear fashion, while female caregivers have a nurturing approach and nest activities inside one another .

Although several clinical reports provide guidelines for managing dementia-related problems (Marchello et al. 1995; Stewart 1995; Teri et al. 2002), little is known about the effects of caregiver management strategies on patient functioning. Evidence from intervention studies (Teri 1999) indicates that caregiver interactions with the patient have an impact on patient behaviour. Lawton's ecological model (Parmelee and Lawton 1990) suggests that people with dementia are even more likely than the average person to be vulnerable to the impact of their environment, because of their diminished competence and function. Thus, the identification of specific caregiver management strategies may 
provide useful information concerning the manifestation of behavioural problems in dementia.

In the present study, we attempted to identify specific caregiver strategies and then investigated whether these are predictors of patient behavioural problems and caregiver distress.

\section{Table 1. Patient and caregiver characteristics}

\begin{tabular}{|c|c|c|}
\hline & $\begin{array}{l}\text { Caregiver } \\
\mathrm{n}=99\end{array}$ & $\begin{array}{l}\text { Patient } \\
n=99\end{array}$ \\
\hline & Mean (SD) & Mean (SD) \\
\hline $\operatorname{Sex}^{1}(m / f)$ & $33 / 66$ & $43 / 56$ \\
\hline Age & $61.9(11.9)$ & $78.2(8.4)$ \\
\hline \multicolumn{3}{|l|}{ Relationship (n, \%) } \\
\hline Spouse & $55(55.6 \%)$ & \\
\hline Child & $44(44.4 \%)$ & \\
\hline Education $^{2}(1-8)$ & $3.4(1.8)$ & \\
\hline Contact per week (hours) & $98.8(69.7)$ & \\
\hline Duration of care (months) & $28.8(27.4)$ & \\
\hline Duration of illness (months) & & $42.6(31.1)$ \\
\hline MMSE-score & & $18.2(4.8)$ \\
\hline \multicolumn{3}{|l|}{ Global Deterioration Scale (GDS) $(\mathrm{N}, \%)$} \\
\hline Mild 3-4 & & $69(69.7 \%)$ \\
\hline Severe 5-6 & & $30(30.3 \%)$ \\
\hline \multicolumn{3}{|l|}{ Dementia diagnosis patient } \\
\hline Alzheimer's disease & & 73 \\
\hline Vascular dementia & & 19 \\
\hline Frontal lobe dementia & & 2 \\
\hline Parkinson's disease & & 3 \\
\hline Mixed (AD/vascular) & & 2 \\
\hline
\end{tabular}

MMSE - Mini-Mental State examination | GDS = Global Deterioration Scale

1. values represent number of males and females | 2. ranging from primary school (1) to university degree (8)

\section{Methods}

\section{Subjects}

Subjects were 99 informal caregivers of ambulatory patients with dementia diagnosed according to DSM-IV criteria (American Psychiatric Association 1994). The present study is part of the Maastricht Study of Behaviour in Dementia (abbreviated as MAASBED). 
MAASBED is a 2-year follow-up study that focuses on the course and risk factors of behavioural and psychological symptoms of dementia (BPSD). Patients were referred by the Memory Clinic of the Academic Hospital Maastricht, or the geriatric division of the Regional Institute for Community Mental Health (RIAGG), Maastricht, the Netherlands. Data on clinical symptoms, neuropsychological testing, laboratory studies and neuroimaging were discussed in plenary clinical rounds to diagnose patients according to DSMIV criteria.

Caregivers were included if they were the primary caregiver and had contact with the patient at least once a week. All patients were living at home at baseline. Informed consent was obtained from all subjects. Patient and caregiver assessments were conducted by independent, clinically experienced, trained psychologists.

Of the 119 informal caregivers participating in MAASBED, 99 agreed to be interviewed at baseline (83.2\%). Caregivers who participated did not differ from those who did not in terms of age, sex, education, length of care, depression, and patient behavioural problems. Subject characteristics are shown in table 1.

\section{Patient measures}

Patient behavioural problems were measured with the NeuroPsychiatric Inventory (NPI; Cummings 1994), a structured interview with the caregiver that evaluates 12 neuropsychiatric symptoms. The total score on each item can range from 1 to 12 and is obtained by multiplying severity ( 1 "mild" to 3 "severe") by frequency (1 "sometimes" to 4 "very often"). Principal component analysis was used to identify behavioural subsyndromes measured by the NPI. This resulted in three components, explaining 55\% of variance in the data (Aalten et al. 2003): (1) a hyperactivity factor, including the symptoms disinhibition, irritability, agitation, euphoria, and aberrant motor behaviour (5 items, Cronbach's alpha = 0.73); (2) a mood/apathy factor including depression, apathy, nighttime behavioural disturbances and eating abnormalities ( 4 items, $\alpha=0.63$ ); and (3) a psychosis factor, including hallucinations and delusions ( 2 items, $\alpha=0.72$ ). The symptom anxiety loaded high on several factors and therefore was not included in the analysis. Total scores for each sub syndrome were computed as the sum of observed NPI item scores for each factor.

The Mini Mental Status Examination (MMSE; Folstein et al. 1975) was used to measure the patient cognitive functioning of the patients. The dependence on others of the patient 
with regard to daily activities was measured with the Interview for Deterioration in Daily living activities in Dementia (IDDD; Teunisse 1995). This questionnaire consists of 20 items reflecting initiative to and actual performance of daily activities. We used a summed score for items concerning actual performance of activities (range 0 to 44) and for items concerning initiative to activities (range 0 to 36 ).

\section{Caregiver measures}

Caregivers completed a semi-structured interview, administered according to a standardized procedure (for further description see 'data analysis' below). Questions in the interview were formulated to elicit information about the way caregivers manage the caregiving situation and how they deal with dementia-related problems.

For each of the 12 BPSD symptoms on the NPI, caregivers rated the level of distress they experienced on a scale from 0 (none) to 5 (extreme). NPI-D score is the sum of these 12 ratings.

Caregiver competence was measured with the Short Sense of Competence Questionnaire (SOC; Vernooij-Dassen et al. 1999). This questionnaire consists of 7 items rated on a 5point scale ( 1 "agree very strongly" to 5 "disagree very strongly"). These items reflect three domains of caregivers' feelings of being capable of caring for a demented person: (1) satisfaction with the demented person as a recipient of care; (2) satisfaction with one's own performance as a caregiver; and (3) consequences of involvement in care for the personal life of the caregiver. The content validity and construct validity have been reported elsewhere (Vernooij-Dassen et al., 1999), Cronbach's alpha in this study is 0.74 .

Depressive symptoms were measured with the Montgomery-Asberg Depression Rating Scale (MADRS; Montgomery and Asberg 1979), a structured interview. Ratings (from 0 to 6) on 10 items were summed.

\section{Data analysis}

The semi-structured interviews were analysed qualitatively to identify caregiver management strategies, independently of other caregiver measures. The interviews were tape-recorded and then transcribed verbatim for analysis. The content of the transcripts was analysed using the Grounded Theory process of constant comparative analysis to identify common themes and issues (Strauss \& Corbin, 1990). This process involved reading and re-reading interviews, selecting and coding data. The process of interpreting and identifying categories involved searching for key themes, noting associations and 
comparing (deviant) cases. Ethnograph version 5 was used for qualitative analysis (Seidel 1998). The analyses resulted in the identification of three strategies. The reproducibility of the results was checked by an independent observer. Agreement of coding between both researchers was 77\% (kappa=0.62). Agreement of coding between both researchers was based on the agreement in allocation of caregivers to one of the three strategy groups by analysing the interviews. Differences in coding were discussed between both researchers to see if consensus could be reached. If both researchers still disagreed the coding of a third independent observer was decisive.

Univariate comparisons were conducted with ANOVA and Chi Square tests. Differences in BPSD (NPI) between the three caregiver management strategy groups were analysed using repeated measures analysis of variance (ANCOVA) with three caregiver strategy groups, sex, relationship (partner versus child) and education (low, medium, high) as between-subjects factors. In this analysis time (NPI at 0, 6 and 12 months) was used as within-subject factor. This analysis was repeated for the three NPI sub-syndromes. Therefore significance level was Bonferonni corrected and set at 0.0125. Results of the ANCOVA for the separate sub-syndromes were verified with logistic regression, because some variables somewhat deviated from a linear distribution. To examine differences in caregiver outcomes, ANCOVA was repeated for caregiver competence (SOC) and caregiver distress (NPI-D). Significance level was Bonferonni corrected and set at $\alpha=.025$. All tests were performed two-tailed.

\section{Results}

\section{Caregiver strategies}

Qualitative data analysis resulted in the identification of three types of caregiver management strategies, based mainly on whether the caregiver accepted the caregiving situation and of dementia-related problems. Caregiver strategies characterized by nonacceptance were termed "non-adapting" ( $n=17)$. Caregiver strategies characterized by acceptance were further subdivided into two different strategies termed "nurturing" $(n=30)$ and "supporting" $(n=52)$. Although some caregivers used aspects of different strategies in conjunction, in all cases consensus was reached between the observers about the most prominent strategy. 


\section{Nurturing}

Nurturing indicates efforts to manage the patient by using a "parent-child approach", in which the patient is "taken by the hand" and is no longer regarded as an equal. In this approach caregivers felt responsible for doing most of the daily chores. They tried to protect the patient or tended to focus on personal care tasks, such as physical assistance with self-care and providing meals. As one wife said,

"I take more care of him. I have always been a caring person, but now I feel that I'm taking care of a child. I don't know how to do it otherwise. But I believe this is the right way. »

\section{Supporting}

Supporting describes efforts to manage the patient by adapting to the patient's level of functioning and by stimulating his or her existing abilities. These caregivers allow the patient to "lead the way". Several aspects of supporting were identified. First, caregivers tried to supervise the activities of the patient, assisting the patient when needed or supporting the patient by discussing things. Second, caregivers tended to be patient with the patient adopting a calm or cautious manner and tried to manage behavioural problems with compliance. Third, caregivers tried to stimulate the patient to undertake physical, social or household activities or they did pleasant activities together. As a husband said,

"I let my wife live her own life. I don't tell her what to do... I don't do that. I let her go her own way, because that's important to her. You have to be careful with these patients and not give her the feeling that I'm constantly watching her. »

Non-adapting

These caregivers were characterized by a lack of understanding of the patient or of acceptance of the situation. They primarily approached the patient with impatience, irritation or anger. They tried to manage behavioural problems by confronting or ignoring the patient, for example by walking away. As a daughter said,

"I don't think I manage her the right way, because sometimes I get irritated ... Recently / said to her that it was better for me to go home, because she started telling stories that weren't true. At that moment I interrupted her and said that it wasn't right what she was telling. I know that's wrong, but I can't help myself. » 
Table 2. Patient and caregiver characteristics per caregiver strategy

\begin{tabular}{|c|c|c|c|c|c|c|}
\hline & $\begin{array}{l}\text { Total } \\
\mathrm{n}=99\end{array}$ & $\begin{array}{l}\text { Nurturing } \\
n=30\end{array}$ & $\begin{array}{l}\text { Supporting } \\
\mathrm{n}=52\end{array}$ & $\begin{array}{l}\text { Non- } \\
\text { adapting } \\
n=17\end{array}$ & $\begin{array}{l}\text { Test } \\
\text { value }\end{array}$ & $P$ value \\
\hline & Mean (SD) & Mean (SD) & Mean (SD) & Mean (SD) & & \\
\hline \multicolumn{7}{|l|}{ Caregiver } \\
\hline \multicolumn{7}{|l|}{$\operatorname{Sex}(n, \%)$} \\
\hline Male & $33(33.3 \%)$ & $5(16.7 \%)$ & $24(46.2 \%)$ & $4(23.5 \%)$ & $\chi^{2}=8.3$ & 0.016 \\
\hline Female & $66(66.7 \%)$ & $25(83.3 \%)$ & $28(53.8 \%)$ & $13(76.5 \%)$ & & \\
\hline \multicolumn{7}{|l|}{ Relationship (n, \%) } \\
\hline Spouse & $55(55.6 \%)$ & $15(50 \%)$ & $33(63.5 \%)$ & $7(41.2 \%)$ & $\chi^{2}=3.1$ & 0.211 \\
\hline Child & $44(44.4 \%)$ & $15(50 \%)$ & $19(36.5 \%)$ & $10(58.8 \%)$ & & \\
\hline \multicolumn{7}{|l|}{ Education level $^{1}(n, \%)$} \\
\hline Low & $37(37.4 \%)$ & $13(43.3 \%)$ & $16(30.8 \%)$ & $8(47.1 \%)$ & $\chi^{2}=10.1$ & 0.038 \\
\hline Medium & $40(40.4 \%)$ & $15(50 \%)$ & $18(34.6 \%)$ & $7(41.1 \%)$ & & \\
\hline High & $22(22.2 \%)$ & $2(6.7 \%)$ & $18(34.6 \%)$ & $2(11.8 \%)$ & & \\
\hline \multicolumn{7}{|l|}{ Contact per week $(n, \%)$} \\
\hline Low & $37(37.4 \%)$ & $14(46.7 \%)$ & $16(30.8 \%)$ & $7(41.2 \%)$ & $\chi^{2}=2.2$ & 0.336 \\
\hline High & $62(62.6 \%)$ & $16(53.3 \%)$ & $36(69.2 \%)$ & $10(58.8 \%)$ & & \\
\hline Duration of care (months, SD) & $28.8(27.4)$ & $30.6(27.4)$ & $28.1(29.1)$ & $27.5(22.2)$ & $F=2.2$ & 0.591 \\
\hline NPI distress & $11.2(10.3)$ & $12.6(12.9)$ & $9.2(8.1)$ & $14.6(10.7)$ & $F=2.3$ & 0.105 \\
\hline Sense of Competence & $23.5(6.3)$ & $22.3(6.2)$ & $25.9(4.8)$ & $18.2(7.1)$ & $F=12.6$ & $<0.001$ \\
\hline MADRS & $8.7(6.3)$ & $9.9(6.3)$ & $7.1(5.4)$ & $11.3(7.6)$ & $F=3.1$ & 0.049 \\
\hline Neuroticism & $30.8(7.1)$ & $32.2(5.5)$ & $28.3(7.1)$ & $36.1(6.7)$ & $F=8.3$ & $<0.001$ \\
\hline \multicolumn{7}{|l|}{ Patient } \\
\hline GDS & $4.1(0.7)$ & $4.2(0.6)$ & $4.1(0.7)$ & $4.1(0.8)$ & $F=0.2$ & 0.862 \\
\hline MMSE & $18.2(4.8)$ & $17.7(3.9)$ & $18.1(5.3)$ & $19.2(4.5)$ & $F=0.5$ & 0.591 \\
\hline NPI total score & $24.9(25.9)$ & $29.7(33.6)$ & $19.7(18.5)$ & $33.1(28.0)$ & $F=2.2$ & 0.113 \\
\hline Mood/apathy & $10.2(10.8)$ & $12.4(13.1)$ & $6.8(9.9)$ & $2.4(4.9)$ & $\chi^{2}=2.1$ & 0.345 \\
\hline Hyperactivity & $6.8(9.9)$ & $9.4(13.7)$ & $3.9(5.2)$ & $11.3(10.8)$ & $\chi^{2}=6.8$ & 0.034 \\
\hline Psychosis & $2.4(4.9)$ & $2.3(5.9)$ & $2.3(4.5)$ & $2.8(4.2)$ & $\chi^{2}=2.2$ & 0.341 \\
\hline \multicolumn{7}{|l|}{ IDDD } \\
\hline Initiative & $21.9(9.8)$ & $19.9(9.3)$ & $22.3(10.1)$ & $24.1(9.5)$ & $F=1.1$ & 0.343 \\
\hline Performance & $20.4(11.1)$ & $24.6(8.7)$ & $18.5(11.6)$ & $18.9(11.8)$ & $F=3.3$ & 0.043 \\
\hline
\end{tabular}

$\mathrm{NPI}=$ Neuropsychiatric Inventory $\mid$ MADRS = Montgomery Asberg Rating Scale $\mid$ GDS = Global Deterioration Scale | MMSE = Mini Mental State Examination | IDDD = Interview of Daily living activities in Dementia

1. educational level was compressed from eight to three levels: low (level 1 and 2), medium (level 3 to 5), and high (level 6 to 8 )

\section{Caregiver strategies: baseline differences}

Differences in patient and caregiver characteristics between the three groups of caregivers are shown in table 2. Fifty-three percent of the caregivers used a supporting management strategy, $30 \%$ a nurturing strategy, and only $17 \%$ a non-adapting strategy. 
Relatively more women than men used a nurturing strategy (25 versus 5) or a nonadapting strategy (13 versus 4). No difference was found in management strategies between spouses and children. Caregivers who used a non-adapting strategy reported a lower sense of competence, more depressive symptoms, and higher levels of neuroticism than caregivers who used the other strategies. Relatively more caregivers who used a supporting strategy were highly educated. Caregivers who used the non-adapting strategy reported more patient hyperactivity symptoms than did caregivers who used the other strategies. There was also a significant difference between the groups in patient performance of daily activities, with caregivers who used the nurturing strategy reporting lower levels of patient activities than caregivers who used the other strategies. However there were no differences in patient initiative to undertake activities or other measures of patient functioning.

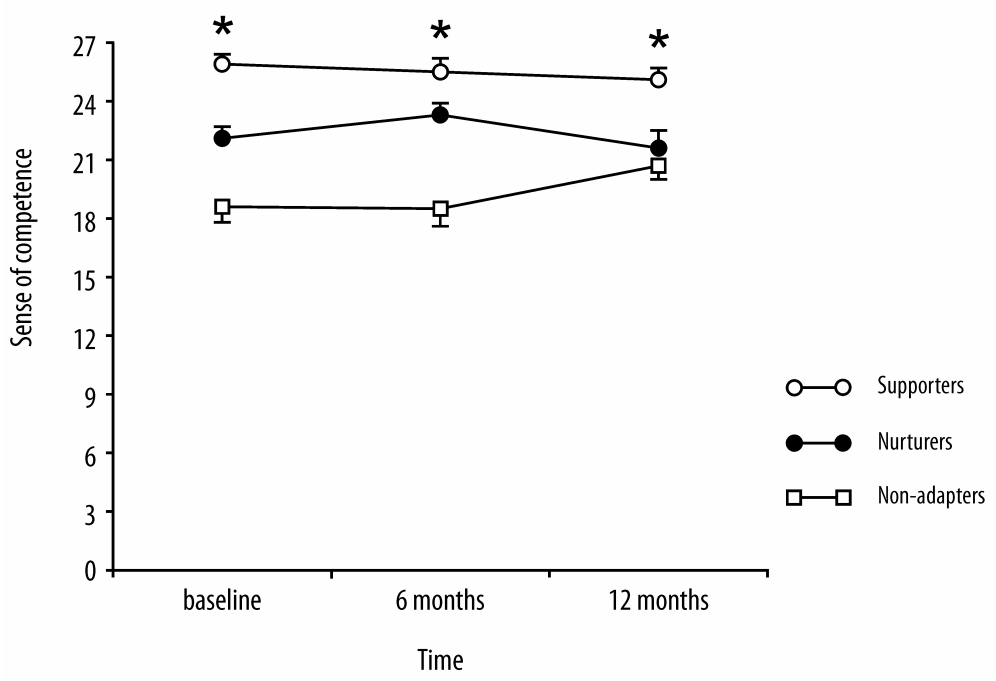

Figure 1. Sense of Competence (mean \pm SEM) in nurturers $(n=23)$, supporters $(n=42)$, and non-adapters $(n=11) \mid * P<0.05$

\section{Caregiver strategies and caregiver functioning over time}

ANCOVA was used to investigate differences in caregiver functioning over time between the three types of strategies. Results for sense of competence showed an overall difference between the groups ( $F=6.1(2,69), p=0.003)$, even after adjusting for baseline dementia severity $(F=5.9(2,68), p=0.004)$. There was a significant difference between the 
caregivers who used a supporting strategy and the caregivers who used a non-adapting strategy $(t=3.4, p=0.001)$, with caregivers who used a non-adapting strategy reporting the lowest sense of competence over time (Fig. 1).

There was no significant overall change in SOC over time ( $F=0.1(2,138), p=0.861)$. Post-hoc comparisons revealed a significant difference in SOC between the three groups at baseline $(F=12.6(2,89), p<0.001)$ and at 6 months follow up $(F=13.2(2,86), p<0.001)$ but not at 12 months $(F=3.8(2,78), p=0.038)$. Groups did not differ with regard to NPI-distress scores $(F=0.248(2,76), p=0.781)$.

\section{Caregiver strategies and patient behaviour}

ANCOVA was performed to investigate differences in patient behaviour over time between the caregivers who used the different management strategies. Results showed no overall difference in BPSD between the groups $\left(F=1.9_{(2,74),} p=0.152\right)$. However, posthoc comparisons revealed a significant difference in BPSD between the groups at 6 months $(F=5.3(2,88), p=0.007)$ and 12 months follow up $(F=4.2(2,83), p=0.019)$, with caregivers who used a non-adapting strategy reporting higher levels of BPSD than caregivers who used a supporting strategy.

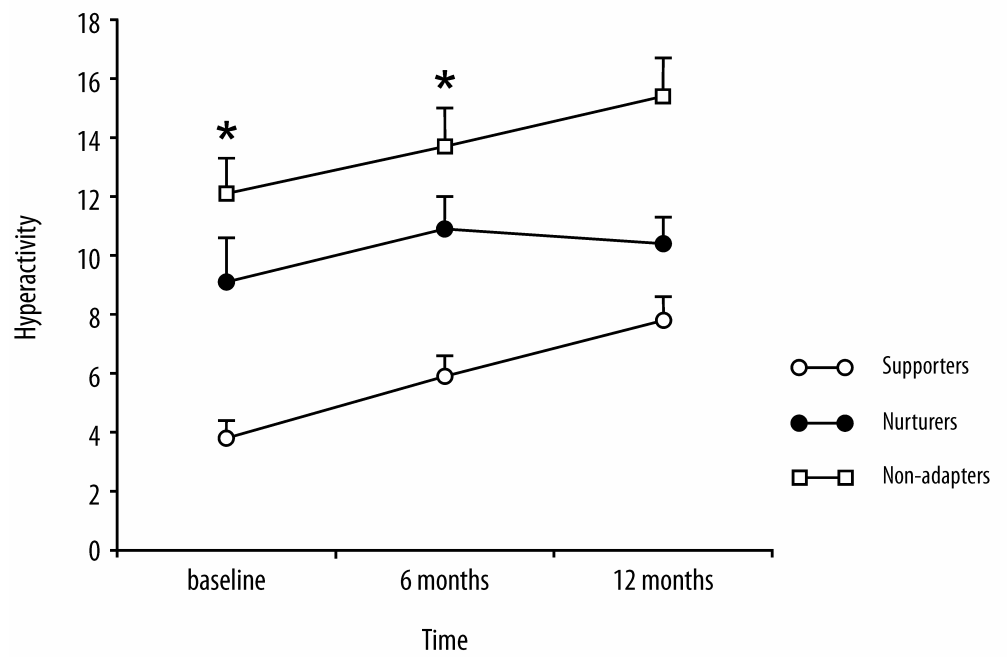

Figure 2. Hyperactivity (mean \pm SEM) in patients of nurturers $(n=23)$, supporters $(n=44)$, and non-adapters $(n=14) \mid * P<0.05$ 
Comparable analyses were performed for the three sub syndromes of BPSD to examine differences in patient mood/apathy, hyperactivity and psychosis between the groups. ANCOVA showed an overall difference in hyperactivity between the groups $(F=4.2(2,76), p$ $=0.018$ ) (Fig.2), with caregivers who used a non-adapting strategy reporting higher levels of hyperactivity than caregivers who used a supporting strategy $(t=2.9, p=0.005)$. There was also a significant increase in hyperactivity over time $(F=4.5(2,148), p=0.015)$. Post-hoc comparisons revealed a significant difference in hyperactivity between the groups at baseline $(F=4.4(2,98), p=0.015)$ and at 6 months $(F=5.4(2,88), p=0.006)$.

ANCOVA for mood/apathy and psychosis showed no overall differences in patient behaviour between the groups (respectively $F=0.7_{(2,74),} p=0.512$ and $F=0.9_{(2,74)}, p=$ 0.380).

\section{Discussion}

The main finding of the study is that caregiver management strategies are associated with BPSD and the caregiver's sense of competence. We were able to identify three types of strategies, namely, nurturing, supporting and non-adapting strategies. While these strategies were identified from interviews independent of other caregiver measures, there were differences between the three management styles in several caregiver characteristics such as sex, education and personality. This indicates that stable caregiver characteristics are important determinants of the way caregivers manage patients.

The finding that male and female caregivers tended to use different care strategies is in line with the study of Fitting and colleagues (Fitting et al. 1986). One explanation for this sex difference in style of caregiving is that it traditionally has been the female's social role to perform nurturing activities for individuals who are unable to care for themselves (Gallicchio et al. 2002). Highly educated caregivers tended to use a supporting strategy, which suggests that these caregivers are better able to adjust to the care demands and patient level of functioning than other caregivers.

Only a few caregivers used the non-adapting strategy. These caregivers also felt less competent at all follow-up times. They were also more neurotic and reported more depressive symptoms at baseline than the other caregivers did, which is similar to the findings of Hinrichsen and Niederehe (1994). These results are also in line with the findings of studies of general coping strategies, which showed that emotional reactive or avoidant ways of responding to problematic situations are associated with more subjective stress (Matsuda 1995; Powers et al. 2002). 
There were some differences between the caregiver groups in patient functioning at baseline. The patients of caregivers who used a nurturing strategy performed fewer daily living activities, which is consistent with these caregivers feeling responsible for doing the daily chores and with their greater focus on personal care tasks. It is possible that patients cared for by caregivers who used a nurturing strategy are less able to perform these daily tasks themselves than other patients. However, the fact that illness severity, in terms of the global deterioration score, was similar between the patient groups suggests that caregivers who used a nurturing strategy took over most of the daily tasks and presumed the patient was less able to perform these tasks themselves.

We also investigated changes in patient behaviour in relation to caregiver management strategies. The caregivers who used a non-adapting strategy reported more patient hyperactivity than did the caregivers who used a supporting strategy.

These results suggest that caregivers who use a non-adapting strategy are more likely to encounter hyperactive behaviour in patients than caregivers who use a supporting strategy. This finding could indicate that hyperactive behaviour in dementia patients is triggered by caregiver interactions with the patient. Caregiver impatience, irritation or anger may result in greater agitation in the patient, which is in line with results of Hamel and colleagues (Hamel et al. 1990), who found that a poorer interpersonal relationship between caregiver and patient resulted in greater patient aggression. The caregivers who used a supporting strategy seemed to be most effective in dealing with patient problems and reduced the risk of hyperactive behaviour. Their effort to adjust to the behaviour and abilities of the patient probably creates a safe environment and minimizes patient frustration, which is in line with findings of Harvath (Harvath 1994).

Baseline differences between the 3 strategy groups suggest that caregiver characteristics such as gender, education and personality are important determinants of caregiver strategies. It is supposed that caregiver strategies subsequently influence patient behaviour. However, caregivers may also tend to use different strategies when problem behaviours are present in the patient. So, there might be a bi-directional pattern of causation. Longitudinal data on caregiver strategies may indicate whether caregivers change their strategy over time. However, baseline results did not show any influence of duration of care on caregiver strategy, which suggests that management strategies do not change in different phases of caregiving. 
Our study has several limitations. One is the relatively small number of caregivers, especially in the group using a non-adapting strategy. It is possible that with larger caregiver groups and therefore an increased statistical power, the tendencies we found would become statistically significant.

A second limitation involves the fact that the treatment of behavioural disturbances in the patient was not taken into account. However, this probably results in an underestimation of the negative impact of caregiver management strategies on patient behaviour, because successful treatment will decrease the severity of the behavioural problems.

A third limitation is the fact that caregiver reports were used to assess patient behavioural symptoms. It may be argued that non-adaptive caregivers provide more negative ratings of patient behaviour, while these actual patient's behaviours may be less severe. However, the finding that non-adaptive caregivers report specific behavioural symptoms (hyperactivity) and not other problem behaviours invalidates this argument.

Finally, in this study caregivers were allocated to one particular strategy. But, some caregivers used aspects of different strategies in conjunction. So, one must take into account that the distinction between the three types of caregivers may be less clear than the discrimination of the three types of strategies. However, the fact that two independent observers identified the same strategy in $77 \%$ of the cases indicates that in most caregivers one strategy is most dominant.

Our results indicate that differences exist among caregivers in the way they adapt to the demands of the caregiving situation. Findings suggest that caregiver characteristics such as sex, education and personality are important determinants of management strategies. Inadequate caregiver management strategies may put caregivers at risk of experiencing a higher burden and may increase patient agitation. Intervention programs should aim at training caregivers to use adequate management strategies, which could increase caregiver competence and decrease patient problem behaviour.

\section{Key points}

- Caregivers differ in their management strategies

- $\quad$ Caregiver sex, education and personality are important determinants of management strategies

- $\quad$ Caregiver management strategies are important in predicting patient hyperactivity and caregiver burden 


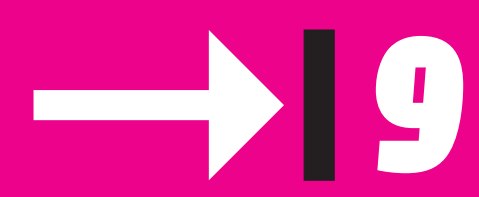

Expressed emotion in the caregiver as predictor of behavioural problems in dementia 


\section{Abstract}

Objective: Caregiver functioning may have an important influence on behavioural problems in dementia patients. Little is known about the impact of caregiver expressed emotion (EE) on negative patient behaviour in dementia. In a prospective design the differential impact of EE on specific behavioural syndromes in patients and the impact of EE on caregiver functioning were investigated.

Methods: Ninety-three patients with dementia and their informal caregivers were followed up for 1 year. EE was assessed using the Five Minute Speech Sample (FMSS) at baseline. Behavioural disturbances in the patient were measured with the NeuroPsychiatric Inventory (NPI). Repeated measures analyses were carried out to investigate the relationship between caregiver $\mathrm{EE}$ and patient hyperactivity, psychosis and mood/apathy.

Results: Caregiver EE was associated with specific patient behavioural problems and caregiver functioning. At baseline and 6 months follow-up high EE was related to patient hyperactivity symptoms and lower feelings of competence in caregivers. No associations were found between high EE and patient mood/apathy or psychosis. Also, no associations were found between high EE and patient behaviour or caregiver functioning at 1 year follow-up. In addition, post-hoc analysis showed an association between high EE and patient institutionalisation.

Conclusions: Results suggest that caregiver-patient interaction influences patient as well as caregiver functioning. There are potential implications of these results for caregiver interventions. Interventions such as psycho-education and teaching of problem-solving strategies should be designed and evaluated to reduce the level of EE in dementia caregivers and subsequently increase caregiver well-being, reduce patient hyperactivity and delay institutionalisation. 


\section{Introduction}

Behavioural problems are a major problem in patients with dementia, and occur in most patients during the course of the disease. These behaviours have important consequences for family caregivers because they are associated with caregiver distress (Donaldson et al. 1998) and negative health effects in caregivers (Hooker et al. 2002). Furthermore, these symptoms are important determinants of nursing home placement (O'Donell et al. 1992; Vugt et al. 2003).

Most studies have focused on the consequences of behavioural problems on caregiver burden. However, attitudes of caregivers may vice versa also affect patient functioning. A poor dyadic interaction can maintain or exacerbate dysfunctional behaviours in the patient (Patterson and Hops 1972; Vitaliano et al. 1993). This interactive aspect as a source of problem behaviour has often been overlooked. Expressed Emotion (EE), an indicator of such an interaction, is defined as the emotional climate between patient and caregiver (Leff and Vaughn 1985). High EE is defined as a critical and hostile attitude and/or emotional over involvement of the patient's caregiver. It has been identified as a risk factor for relapse in schizophrenic and uni- and bipolar depressed patients (Butzlaff 1998; Hooley et al. 1986; Parker and Hadzi-Pavlovic 1990). EE may also be an important risk factor for an adverse course of behavioural problems in dementia patients (Magana et al. 1986). EE has been studied in caregivers of dementia patients, where high EE levels were found to be associated with caregiver distress (Bledin et al. 1990; Gilhooly and Whittick 1989; Tarrier et al. 2002). Few studies have examined caregiver EE in relation to patient behaviour in dementia. In a longitudinal study Vitaliano and colleagues (1993) found that high levels of caregiver EE were predictive of restless behaviour in Alzheimer patients over time. In this study, high EE levels were not related to cognitive or functional problems in the patient. Wagner and colleagues (1997) however found no relation between EE levels in caregivers of patients with Alzheimer's disease and patient depression and behavioural problems. The discrepancy between these two studies may be due to differences in sample characteristics, such as differences in sample size (79 versus 57), or in the assessment of behavioural problems (Screen for Caregiver Burden versus Hamilton Depression Rating Scale and Revised Memory and Behaviour Problem Checklist). However, it may also be that the impact of EE varies for different behavioural symptoms or groups of symptoms, for instance one may conceive that patient restlessness may be more susceptible to caregiver affect than patient feelings of depression. However, these two studies did not look at the differential effect of EE on specific patient behavioural symptoms. 
The current study used a large sample and a 1-year prospective design to examine the differential impact of EE on specific behavioural syndromes in patients. In addition, the impact of EE on caregiver functioning was investigated.

\section{Methods}

\section{Subjects}

Subjects were informal caregivers of ambulatory patients with dementia according to DSM-IV (American Psychiatric Association 1994). The present study is part of the MAAstricht Study of BEhaviour in Dementia (abbreviated as MAASBED). MAASBED is a 2year follow-up study that focuses on the course and risk factors of behavioural and psychological symptoms of dementia (BPSD). Patients and their caregivers are seen at 6month intervals. Patients were referred by the Memory Clinic of the Academic Hospital Maastricht, or the geriatric division of the Regional Institute for Community Mental Health (RIAGG), in Maastricht. Caregivers were included if they were the primary caregiver and had at least once a week contact with the patient. At baseline all patients were living at home. Informed consent was obtained from all subjects. Of the 119 informal caregivers participating in MAASBED, 111 agreed to be interviewed at baseline (93.3\%). Caregivers who participated did not differ from those who did not in terms of age, sex, education, length of care, depressive symptoms, or characteristics of the patients cared for (dementia severity, patient behavioural problems). The mean caregiver age was $63.3(S D=12.1)$ and their mean educational level was secondary school (mean $=3.5 ; S D=1.8$; ranging from 1primary school to 8-university) (see table 1). There were 38 males and 73 females. Fiftyseven (51.4\%) caregivers were spouses, 46 (41.4\%) children and 8 (7.2\%) other relatives or friends of the patient. The mean duration of care was 28.2 months $(S D=26.2)$, and the mean contact hours per week with the patient was 92.1 ( $S D=70.7)$. There were 45 male patients and 66 female patients. The mean duration of illness was 42.3 months ( $S D=30.6)$ and mean MMSE score was 18.1 (SD = 4.7). Eighty-three patients had Alzheimer's disease, 19 vascular dementia, 2 Frontotemporal dementia, 3 Parkinson's disease, 1 primary progressive dementia, and 3 mixed dementia (AD/vascular). Patients used the following medication at baseline: 11 patients (9.8\%) used neuroleptics, 30 patients (26.8\%) antidepressants, 26 (23.2\%) benzodiazepines, 12 (10.8\%) cholinesterase inhibitor.

The current study refers to the 1-year follow-up. Missing values in the follow-up were due to refusal $(n=9)$ or death of the patient $(n=9)$. Caregivers lost to follow-up were relatively older $(t=2.2, p=0.028)$ and more depressed $(t=2.2, p=0.005)$. There were no other 
differences in demographic variables, EE classification or baseline scores of the dependent variables between the follow-up sample and the loss to follow-up group.

\section{Patient measures}

Patient behavioural problems were measured with the NeuroPsychiatric Inventory (NPI) (Cummings 1994), a structured interview with the caregiver that evaluates 12 neuropsychiatric symptoms. The total score on each item can range from 1 to 12 and is obtained by multiplying severity ( 1 "mild" to 3 "severe") by frequency ( 1 "sometimes" to 4 "very often"). Principal component analysis of the NPI identified three behavioural subsyndromes: (a) a hyperactivity factor, including the symptoms disinhibition, irritability, agitation, euphoria, and aberrant motor behaviour (5 items, Cronbach's alpha $=0.73$ ); (b) a mood/apathy factor including depression, apathy, night-time behavioural disturbances and eating abnormalities ( 4 items, $\alpha=0.63$ ); and (c) a psychosis factor, including hallucinations and delusions ( 2 items, $\alpha=0.72$ ) (Aalten et al. 2003). Total scores for each sub syndrome were computed as the sum of observed NPI item scores for each factor.

The Mini Mental Status Examination (MMSE) (Folstein et al. 1975) was used to measure patient cognitive functioning. Patient dependency with regard to daily activities was measured with the Interview for Deterioration in Daily living activities in Dementia (IDDD) (Teunisse 1995). This questionnaire consists of 20 items reflecting initiative to and actual performance of daily activities. We used a summed score for items concerning actual performance of activities (range 0 to 44) and for items concerning initiative to activities (range 0 to 36).

\section{Caregiver measures}

Level of caregiver Expressed Emotion was assessed by the Five-Minute Speech Sample (FMSS) (Magana et al. 1986) at baseline. Caregivers were asked to speak without interruption for five minutes, describing their spouse/parent or other relative and how they got along together. The speech samples were audiotaped and transcribed verbatim. Two qualified raters coded the transcripts using the guidelines described for coding EE (Magana et al. 1986). They rated the number of critical comments, the amount of emotional over involvement (EOI), the initial statement, and the relationship between patient and caregiver, regardless if changes in the patient were due to the dementia. Caregivers were classified as 'high-EE' if they scored on the critical scale and/or on the EOI scale, otherwise they were rated as 'low-EE'. In the low EE group caregivers were rated as 
'borderline-EOI' or 'borderline-critical' when there were some indications for a high EE score, but it did not fulfil the high EE criteria. In order to assess the inter-rater reliability, twelve interviews were randomly selected and rated by two other experienced blind raters $(A H, P D)$ to assess reliability and consistency. The inter-rater reliability between these raters and the two qualified raters was $100 \%$.

For each of the 12 behavioural symptoms on the NPI, caregivers rated the level of distress they experienced on a scale from 0 (none) to 5 (extreme). NPI-Distress score is the sum of these 12 ratings (range $0-60$ ).

Caregiver's subjective competence was measured with the Short Sense of Competence Questionnaire (SOC) (Vernooij-Dassen et al. 1999). This questionnaire consists of 7 items rated on a 5-point scale (1 "agree very strongly" to 5 "disagree very strongly"; range 7-35). These items reflect three domains of caregivers' feelings of being capable of caring for a demented person: (a) satisfaction with the demented person as a recipient of care; (b) satisfaction with one's own performance as a caregiver; and (c) consequences of involvement in care for the personal life of the caregiver. The content validity and construct validity have been reported elsewhere (Vernooij-Dassen et al. 1999). Cronbach's alpha in this study is 0.74 .

Depressive symptoms were measured with the Montgomery-Asberg Depression Rating Scale (MADRS) (Montgomery and Asberg 1979), a structured interview administered by the clinician. Ratings (from 0 to 6) on 10 items were summed (range 0-60).

\section{Data analysis}

The low and high EE groups were compared at baseline in patient and caregiver characteristics using t-tests, Mann-Whitney $U$ tests and Chi-square tests. Square root transformations were used to normalise distributions. Repeated measures analysis of covariance (ANCOVA) was used to test the difference between high and low caregiver EE (between factor with 2 levels) at baseline in behavioural symptoms in the patient at baseline, 6 months, and 12 months follow-up (within factor with 3 levels). Four dependent variables were used: NPI total score, NPI mood/apathy, NPI hyperactivity and NPI psychosis. Therefore significance level was Bonferroni corrected and set at 0.013 . Caregiver gender, relationship with the patient (spouse/other) and contact hours were included in the model.

This analysis was repeated to test the difference between high and low caregiver EE at baseline in caregiver distress at baseline, 6 months, and 12 months follow-up. Three 
dependent variables were used: NPI distress, Sense of Competence and MADRS depressive symptoms. MADRS was only assessed at baseline and 12 months follow-up. Significance level was Bonferroni corrected and set at $\alpha=.025$. All tests were performed two-tailed.

\section{Results}

\section{Expressed Emotion classification}

Seventy-six (63.9\%) caregivers were classified as low EE and 35 (29.4\%) caregivers were classified as high EE. In the high EE group 16 (45.7\%) caregivers were critical, 12 (34.3\%) caregivers were emotionally over involved, and 7 (20.0\%) caregivers were both critical and emotionally over involved. In the low EE group 14 (18.4\%) caregivers were borderlinecritical and 8 (10.5\%) caregivers were borderline-emotionally over involved.

\section{Expressed Emotion and baseline group differences}

First, the relationship between caregiver EE and other baseline caregiver characteristics was examined (table 1). There were no differences between high and low EE caregivers in age, education, contact hours with the patient, relationship to the patient, length of caregiving or caregiver depressive symptoms (all $p$ values were higher than 0.109). However, the high EE caregivers reported significantly higher levels of emotional distress and felt less competent compared with the low EE caregivers.

In addition, patient characteristics were examined at baseline in relation to caregiver EE status. There were no differences between the high and low EE groups in patient behavioural problems, disease severity, cognitive status or functional impairment. There were also no differences between high and low EE groups in patient medication use (all $p$ values were higher than 0.237). 
Table 1. Patient and caregiver characteristics per EE classification

\begin{tabular}{|c|c|c|c|c|c|}
\hline & $\begin{array}{l}\text { Total } \\
n=111\end{array}$ & $\begin{array}{l}\text { EE low } \\
n=76\end{array}$ & $\begin{array}{l}\text { EE high } \\
n=35\end{array}$ & $\begin{array}{l}\text { Test } \\
\text { value }\end{array}$ & $P$ value \\
\hline \multicolumn{6}{|l|}{ Caregiver } \\
\hline \multicolumn{6}{|l|}{$\operatorname{Sex}(n, \%)$} \\
\hline Male & $38(34.2 \%)$ & $29(38.2 \%)$ & $9(25.7 \%)$ & $\chi^{2}=1.6$ & 0.199 \\
\hline Female & $73(65.8 \%)$ & $47(61.8 \%)$ & $26(74.3 \%)$ & & \\
\hline Age (SD) & $63.3(12.1)$ & $63.9(13.2)$ & $62.1(9.3)$ & $\mathrm{t}=0.7$ & 0.482 \\
\hline \multicolumn{6}{|l|}{ Relationship (n, \%) } \\
\hline Spouse & $57(51.4 \%)$ & $40(52.6 \%)$ & $17(48.6 \%)$ & $\chi^{2}=0.5$ & 0.794 \\
\hline Child & $46(41.1 \%)$ & $30(39.5 \%)$ & $16(45.7 \%)$ & & \\
\hline Other & $8(7.2 \%)$ & $6(7.9 \%)$ & $2(5.7 \%)$ & & \\
\hline \multicolumn{6}{|l|}{ Education level ${ }^{1}(\mathrm{n}, \%)$} \\
\hline Low & $39(35.1)$ & $26(34.2 \%)$ & $13(37.1 \%)$ & $\chi^{2}=4.4$ & 0.109 \\
\hline Medium & $46(41.4)$ & $28(36.8 \%)$ & $18(51.4 \%)$ & & \\
\hline High & $26(23.4)$ & $22(29.0 \%)$ & $4(11.4 \%)$ & & \\
\hline \multicolumn{6}{|l|}{ Contact per week $(\mathrm{n}, \%)$} \\
\hline Low & $46(41.4 \%)$ & $31(40.8 \%)$ & $20(57.1 \%)$ & $\chi^{2}=0.04$ & 0.837 \\
\hline High & $65(58.6 \%)$ & $45(59.2 \%)$ & $15(42.9 \%)$ & & \\
\hline Duration of care (months, SD) & $28.2(26.2)$ & $26.8(26.3)$ & $31.1(26.1)$ & $t=-0.8$ & 0.426 \\
\hline NPI distress & $11.2(10.2)$ & $9.5(8.7)$ & $14.6(12.2)$ & $t=-2.1$ & 0.033 \\
\hline Sense of Competence & $23.7(6.1)$ & $24.8(5.7)$ & $21.4(6.1)$ & $t=2.9$ & 0.005 \\
\hline MADRS & $8.3(6.3)$ & $8.1(6.0)$ & $9.0(6.8)$ & $t=-0.8$ & 0.431 \\
\hline \multicolumn{6}{|l|}{ Patient } \\
\hline Age (SD) & $78.6(8.4)$ & $78.9(8.4)$ & $78.0(8.3)$ & $t=0.5$ & 0.593 \\
\hline \multicolumn{6}{|l|}{$\operatorname{Sex}(n, \%)$} \\
\hline Male & $35(31.5 \%)$ & $29(38.2 \%)$ & $16(45.7 \%)$ & $\chi^{2}=0.6$ & 0.451 \\
\hline Female & $76(68.5 \%)$ & $47(61.8 \%)$ & 19 (54.3\%) & & \\
\hline GDS & $4.1(0.7)$ & $4.1(0.7)$ & $4.1(0.6)$ & $t=-0.2$ & 0.812 \\
\hline Illness duration (months, SD) & $42.3(30.6)$ & $38.8(29.9)$ & $49.6(31.0)$ & $t=-1.7$ & 0.085 \\
\hline MMSE & $18.1(4.7)$ & $17.8(4.7)$ & $18.7(4.5)$ & $t=-0.9$ & 0.325 \\
\hline NPI total score & $22.0(22.4)$ & $20.1(20.4)$ & $26.1(26.0)$ & $t=-1.2$ & 0.237 \\
\hline \multicolumn{6}{|l|}{ IDDD } \\
\hline Initiative & $22.5(9.7)$ & $22.9(9.7)$ & $21.6(9.9)$ & $t=0.6$ & 0.519 \\
\hline Performance & $19.9(10.8)$ & $19.9(10.9)$ & $19.9(10.6)$ & $t=0.01$ & 0.994 \\
\hline
\end{tabular}

$\mathrm{NPI}=$ Neuropsychiatric Inventory | MADRS = Montgomery Asberg Rating Scale | GDS = Global Deterioration Scale MMSE = Mini Mental State Examination | IDDD = Interview of Daily living activities in Dementia

1. educational level was compressed from eight to three levels: low (level 1 and 2), medium (level 3 to 5), and high (level 6 to 8) 


\section{Expressed Emotion as predictor of patient behaviour}

Repeated measures analysis of covariance (ANCOVA) was performed to investigate differences in patient behaviour between the high and low EE groups. Results showed no significant overall difference in general levels of behavioural symptoms (total NPI) between the groups $(F=0.5(1,88), p=0.487)$. There was also no significant time by group interaction effect $(F=1.9(2,176), p=0.147)$

ANCOVA was repeated for the three behavioural sub-syndromes to examine differences in patient mood/apathy, hyperactivity, and psychosis. Regarding mood/apathy and psychosis, results showed no difference in patient symptoms between the groups (respectively $F=0.02_{(1,88),} P=0.880$ and $\left.F=0.001_{(1,87),} P=0.969\right)$. Results for hyperactivity showed no overall difference between the groups $(F=2.9(1,87), p=0.094)$, or a change in hyperactivity over time $(F=2.9(2,174), p=0.059)$.

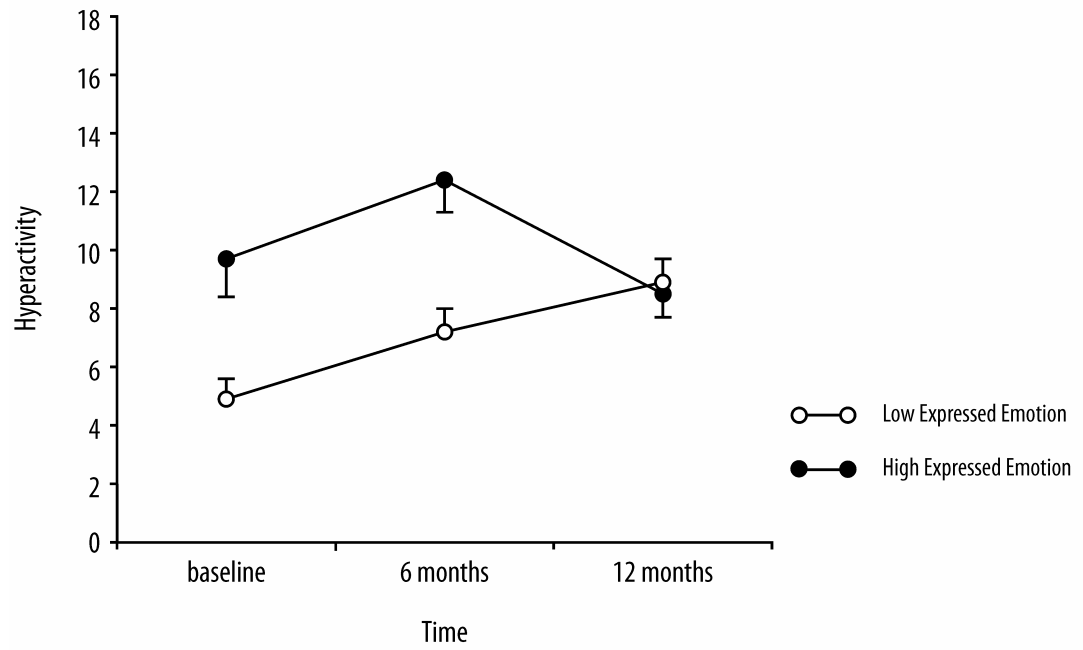

Figure 1. Patient hyperactivity (mean \pm SEM) in low EE $(n=63)$ and high EE $(n=30)$ groups at baseline, 6 months and 12 months follow-up

However, as figure 1 shows there was a decrease in symptoms in the high EE group at 1 year follow-up. To test the difference in hyperactivity between both groups in the first 6 months follow-up, a post-hoc ANCOVA was performed for only baseline and 6 months follow-up. This analysis indeed showed a significant between groups effect, with higher levels of hyperactivity in patients of high $E E$ caregivers ( $F=8.8(1,97), p=0.004)$. We hypothesised that the fact that some of the patients were institutionalised during the 
course of the follow-up could have reduced the association between caregiver EE and patient functioning at 12 months. Therefore a second post-hoc ANCOVA was performed, excluding the patients who were institutionalised during the 1-year follow-up ( $n=52)$. This indeed showed a significant between groups effect, with higher levels of hyperactivity in patients of high EE caregivers over all three measurements $(F=6.4(1,45), p=0.015)$.

In addition, we hypothesised that patients of caregivers in the high EE group were more often institutionalised during the 1 year follow-up than patients of caregivers in the low EE group. A post-hoc logistic regression analysis, adjusted for patient gender, relationship and contact hours, showed that patients of high EE caregivers were indeed more often institutionalised at 1 year follow-up than patients of low EE caregivers (odds ratio $=2.5$, $95 \% \mathrm{Cl}=1.0-6.0$; Wald $\chi^{2}=4.2, \mathrm{df}=1, \mathrm{p}=0.040$ ).

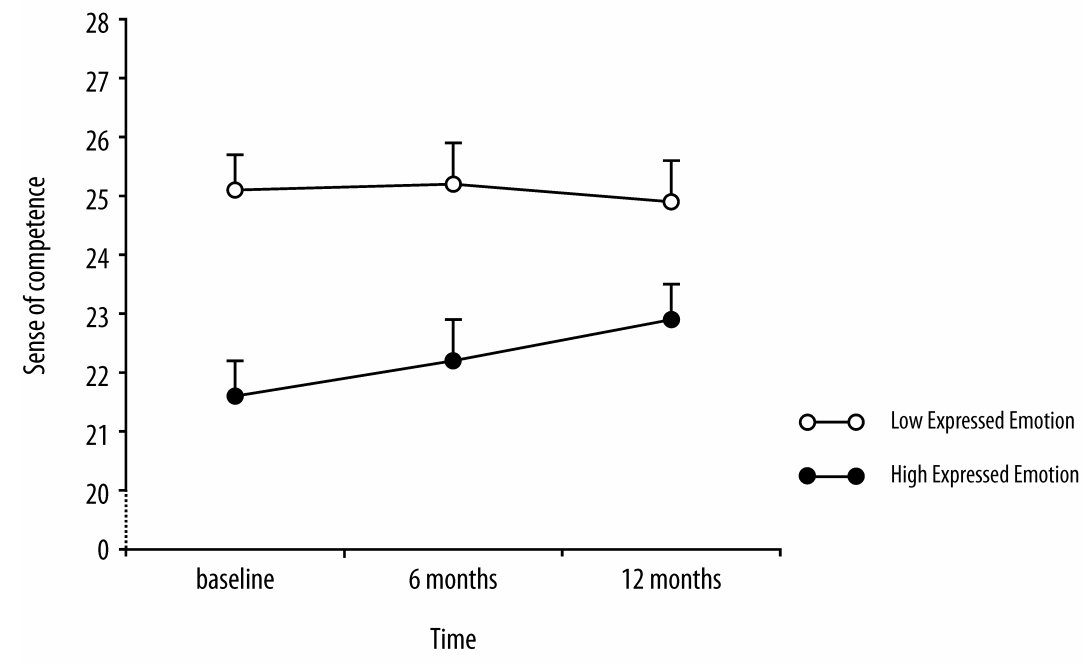

Figure 2. Caregiver sense of competence (mean \pm SEM) in low EE $(n=56)$ and high EE (n=25) groups at baseline, 6 months and 12 months follow-up

\section{Expressed Emotion as predictor of caregiver functioning}

One-year follow-up data were available for 93 (84\%) caregivers for the rating of NPIdistress, for 83 (75\%) caregivers for the rating of MADRS, and for 81 (73\%) caregivers for the rating of SOC. ANCOVA was used to investigate differences in caregiver functioning over time between the high and low EE group. There were no differences between both groups concerning depressive symptoms ( $F=0.002(1,76), p=0.963)$ or distress related to 
patient behaviour ( $F=0.06(1,87), p=0.811)$. Regarding feelings of competence, there was also no significant difference over time between the groups $(F=3.6(1,75), p=0.063$ ) (figure 2). Again, a post-hoc ANCOVA was performed for measurements at baseline and 6 months follow-up showing significant higher feelings of competence in low EE caregivers $(F=8.8(1,90), p=0.004)$.

\section{Discussion}

The main results of the present study are that high levels of EE were present in $29.4 \%$ of the caregivers. At baseline and 6 months follow-up high EE was related to patient hyperactivity symptoms and lower feelings of competence in caregivers. No associations were found between high EE and patient mood/apathy or psychosis. Also, no associations were found between high EE and patient behaviour or caregiver functioning at 1 year follow-up. Patients of high EE caregivers were more likely to become institutionalised during 1 year follow-up than patients of low EE caregivers.

The finding that EE is related to patient hyperactivity is consistent with the results of Vitaliano and colleagues (1993). In their study one overall measure of negative patient behaviour was used, including hyperactive behaviours, paranoia, and wandering. However, they did not differentiate between specific behavioural symptoms. In the current study, patient psychosis and mood/apathy symptoms were also taken into account. However, our results showed no association between these symptoms and EE ratings. This is in line with the findings of Wagner and colleagues (1997), in which caregiver EE was unrelated to patient depression. Our findings indicate that EE is specifically related to patient hyperactivity and is unrelated to other patient behaviour. However, at one year follow-up differences in hyperactivity symptoms were no longer present due to lower levels of hyperactivity symptoms in the high EE group. One explanation could be a possible successful treatment of patient symptoms that reduced patient hyperactivity, however there were no differences in patient medication use at 12 months follow up (all p-values were higher than 0.580). Secondly, the fact that some of the patients were institutionalised during the course of the follow-up is likely to have influenced levels of hyperactivity and probably reduced the interaction between caregiver and patient. Performing the analysis with a subgroup of patients who were living at home during the 1-year follow-up indeed showed also higher levels of hyperactivity in patients of high EE caregivers at 1-year follow-up. Finally, baseline levels of EE may change during the course of the dementia. Caregivers with high EE levels at baseline may have learned to adapt to the caregiving situation, resulting in lower levels of 
EE at later stages of the disease and subsequent lower levels of hyperactivity in the patient. In addition, since the observed association between patient hyperactivity and caregiver EE is already present at baseline, we can not determine temporal precedence. There might be a bi-directional pattern of causation between EE and patient hyperactivity, with lower levels of hyperactivity resulting in lower levels of EE. Repeated EE measurement will indicate whether levels of EE are stable over time or indeed change during the course of the disease.

Besides, an increase in patient hyperactive behaviour in the high EE group, we also found that these patients were more likely to become institutionalised during follow-up. These findings are clinically important, because they might indicate that high EE levels are related to poorer outcome. However, we can not preclude that patients with high hyperactivity levels are more likely to be institutionalised and are also more likely to invoke high levels of EE in their caregivers.

EE was also investigated in relation to caregiver functioning. Results showed that high EE caregivers felt less competent than low EE caregivers during the first 6 months follow-up. This finding is consistent with other studies that have found associations between EE and caregiver well-being (Gilhooly and Whittick 1989). However, in the current study no significant associations were found between EE and caregiver depressive feelings or distress, when adjusting for relevant covariates. This finding seems to be in contrast with studies that have observed an association between depressive feelings or distress and EE in dementia caregivers (Vitaliano et al. 1993; Wagner et al. 1997). However, these studies did not adjust for covariates, such as contact hours between patient and caregiver. We did find higher levels of caregiver distress in the high EE group at baseline when these covariates were not taken into account.

In addition, there were no longer differences in feelings of competence between the high and low EE caregivers when the one year follow-up was included. Again, this could be explained by adaptation of the caregiver to the caregiving situation or by a successful treatment of patient behavioural symptoms.

Our study has some limitations. First, caregiver reports were used to assess patient behavioural symptoms. It may be argued that high EE caregivers provide more negative ratings of patient behaviour, while these actual patient's behaviours may be less severe. However, the finding that high EE is associated with reports of specific behavioural 
symptoms (hyperactivity) and not with other problem behaviours invalidates this argument.

Second, the treatment of behavioural disturbances in the patient was not taken into account in the analysis. However, this probably results in an underestimation of the association between EE and patient behaviour, because successful treatment will diminish problem behaviours and therefore reduce the negative impact of EE on the course of these symptoms. Furthermore, it is not expected that the impact of treatment differs between the EE groups because there were no differences between the groups in patient medication use during follow-up (all p-values were higher than 0.237).

Results of this study suggest that caregiver-patient interaction influences negative behaviours in the patient and caregiver functioning. Findings indicate that high caregiver EE is specifically associated with higher levels of patient hyperactivity, lower feelings of caregiver competence and a greater chance of patient institutionalisation. These results indicate that caregiver interventions that can reduce levels of EE may increase caregiver well-being, reduce patient behavioural problems, and delay patient institutionalisation. Psycho-educational skill training has been found to improve the affective state and the type of coping strategies used by caregivers of dementia patients (Coon et al. 2003). Therefore, psycho-education and teaching of problem-solving strategies could be an effective means to reduce the level of EE in dementia caregivers, as has been shown in caregivers of schizophrenia and bipolar patients (Berkowitz et al. 1990; Honig et al. 1997).

\section{Key points}

- High EE is reported in $29.4 \%$ of the caregivers

- High EE is associated with high levels of patient hyperactivity and lower feelings of caregiver competence

- High EE is associated with patient institutionalisation 
Chapter 9. Expressed emotion in the caregiver as predictor of behavioural problems in dementia 


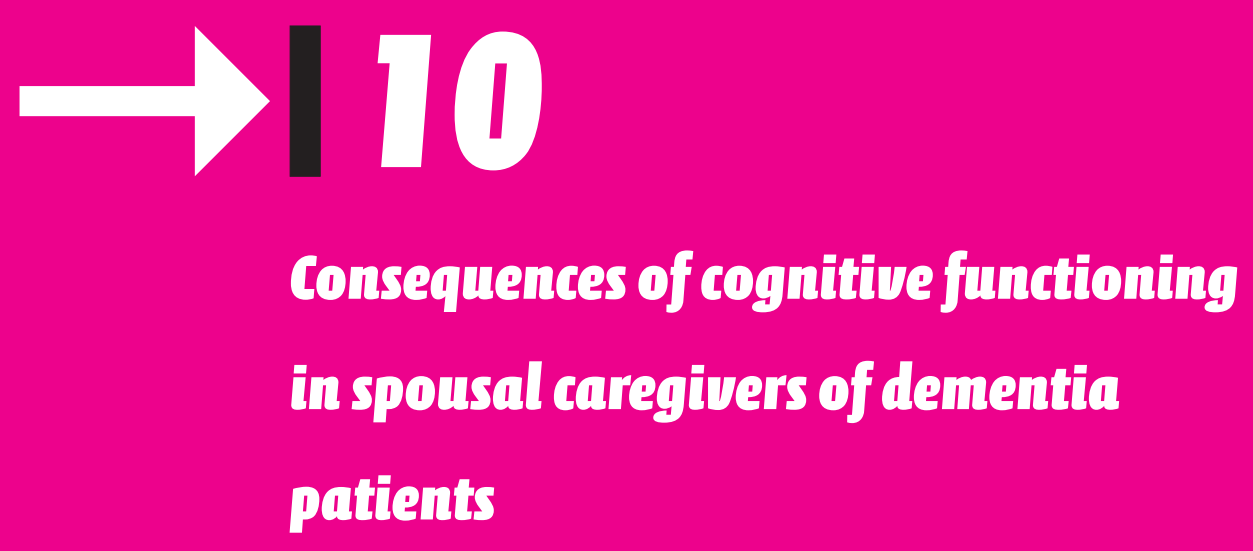

Marjolein E de Vugt, Jelle Jolles, Liesbeth van Osch, Fred Stevens, Pauline Aalten, Richel Lousberg, Frans RJ Verhey 


\section{Abstract}

Objective: Spousal caregivers of dementia patients are usually of the same age as the patient and therefore at risk for age-related cognitive decline. Impaired cognitive functioning in caregivers may have profound implications for patient and caregiver. In a prospective design the impact of caregiver cognitive functioning on behavioural functioning in the patient and caregiver feelings of competence were investigated.

Methods: Cognitive functioning in 54 spousal caregivers was compared to 108 age-, sex-, and education matched controls. Furthermore, patients with dementia and their spousal caregivers were followed-up for 1 year. Behavioural disturbances in the patient were measured with the NeuroPsychiatric Inventory (NPI). Repeated measures statistical analyses were carried out to investigate the relationship between caregiver cognitive functioning and patient behavioural problems.

Results: Spousal caregivers of dementia patients had lower levels of cognitive functioning than control subjects. Caregivers performed significantly worse on measures of general cognitive functioning, speed of information processing, and verbal memory. Low performance on a measure of verbal memory was related to a decrease in caregiver subjective competence and an increase in patient behavioural symptoms, in particular hyperactivity symptoms.

Conclusions Results show sub-optimal cognitive functioning in spousal caregivers, compared to matched controls. Reduced cognitive functioning in caregivers was associated with higher levels of patient hyperactivity and lower feelings of caregiver competence. These results indicate that screening for cognitive impairment of spousal caregivers may be helpful, because reduced cognitive functioning may affect the ability to provide adequate care for the patient. 


\section{Introduction}

A vast majority of dementia patients is cared for at home by their spouse. These spousal caregivers often provide years of extensive care for their partner and must be able to adapt to a continuously changing and demanding situation. The quality of life of both patient and caregiver, and the ability to provide care at home are greatly dependent upon the ability of the caregiver to adequately adapt and respond to the problems and needs of the patient. This ability to care for a demented partner may be compromised by cognitive impairments in spousal caregivers themselves. However, cognitive status in spousal caregivers of dementia patients has hardly been assessed in studies on caregiver functioning.

Spousal caregivers are usually of the same age as the patients and therefore at risk for some age-related cognitive decline. For example, information processing usually becomes slower and less efficient with age (Jolles 1986). In addition, caregivers are likely to experience prolonged elevated levels of stress, which make them particularly vulnerable for cognitive deterioration, such as memory problems (Lupien et al. 1994). As a consequence, the elderly caregiver may not be able to respond accurately to the novel and complex problems they are confronted with in the caregiving process. In such highly stressful and demanding situations, suboptimal cognitive functioning in the caregiver may be related to decreased competence to provide adequate care. Furthermore, it may also affect patient functioning, as especially behavioural problems - in contrast to cognitive impairment -, seem to be sensitive to patient-caregiver interaction. For example, caregiver management strategies have been found to affect behavioural problems in the patient, in particular hyperactivity symptoms (Vugt et al. 2004). However, the impact of cognitive problems in spousal caregivers on patient behaviour has not been studied yet. Cognitive functioning in elderly caregivers at all has hardly been documented. Only two studies were found that addressed this issue. Boucher and colleagues (1996) found that patients of spousal caregivers who scored below the cut-off of a cognitive screening test used fewer community resources and experienced difficulties with medication compliance. This study did not include caregiver educational level as a possible confounder in the relationship between cognitive status and caregiver skills. In a study of Caswell and colleagues (2003) it was found that spousal caregivers have lower levels of complex attention and speed of information processing than non-caregivers, due to chronic stress. However, these studies did not focus on consequences of compromised cognitive functioning on patient and caregiver functioning. 
The aim of the present study is to examine the cognitive functioning of spousal dementia caregivers, and to explore the consequences for patient and caregiver functioning. A prospective design is used to investigate the hypothesis that impaired caregiver cognitive functioning is related to lower levels of caregiver competence and higher levels of patient behavioural problems.

\section{Methods}

\section{Subjects}

Subjects were spousal caregivers of ambulatory patients with dementia according to DSM-IV (American Psychiatric Association 1994). The present study is part of the MAAstricht Study of Behaviour in Dementia (abbreviated as MAASBED). MAASBED is a $2-$ year follow-up study that focuses on the course and risk factors of behavioural and psychological symptoms of dementia (BPSD). Patients and their caregivers are seen at 6month intervals. Patients were referred by the Memory Clinic of the Academic Hospital Maastricht, or the geriatric division of the Regional Institute for Community Mental Health (RIAGG), Maastricht, the Netherlands. Caregivers were included if they were the primary caregiver and had contact with the patient at least once a week. At baseline all patients were living at home. Informed consent was obtained from all subjects. Of the 119 informal caregivers participating in MAASBED, 64 were spouses, of whom 54 agreed to undergo a neuropsychological examination at baseline (84.4\%). Caregivers who participated were significantly younger than non-participators ( $t=2.2, p=0.030$ ). There were no differences between the groups in caregiver sex, education, length of care, depressive symptoms, dementia severity or patient behavioural problems. The mean caregiver age was 68.4 (SD $=8.5)$ and their mean educational level was low $(M=1.8, S D=0.8$; ranging from 1-primary school to 8-university). There were 22 males (40.7\%) and 32 females (59.3\%). At baseline, the mean duration of care was 27.9 months $(S D=25.5)$, and the mean contact hours per week with the patient was $153.6(S D=14.1)$. There were $33(61.1 \%)$ male patients and 21 (38.9\%) female patients with a mean MMSE score of 18.4 (SD = 5.3). Forty-one patients had Alzheimer's disease, 7 vascular dementia, 2 Frontotemporal dementia, 2 Parkinson's disease, 1 primary progressive aphasia, and 1 mixed dementia (AD/vascular), according to regular criteria. Caregivers were matched with 108 control subjects for age, sex and level of education. Control subjects were selected from a large pool of healthy controls, which was collected for use in the MAastricht Aging Study (MAAS; Jolles et al. 1995), a longitudinal investigation on the determinants and consequences of pathological and 
successful aging with respect to cognitive functioning. The participants enrolled in MAAS were selected from a register of 15 general practitioners in the South of the Netherlands. Subjects were excluded if there were any active or inactive medical conditions that might interfere with normal cognitive function or psychosocial contra-indications (e.g. actual major life events). More details on the MAAS population can be found elsewhere (Jolles et al. 1995).

The current study refers to the 1-year follow up. Missing values in the follow up were due to refusal $(n=11)$ or death of the patient $(n=5)$. Caregivers lost to follow-up did not differ from those who were not, in terms of age, sex, education, contact hours, length of care, disease duration, depressive symptoms, feelings of competence, patient cognitive or behavioural problems.

\section{Patient measures}

Patient behavioural problems were measured with the NeuroPsychiatric Inventory (NPI; Cummings 1994), a structured interview with the caregiver that evaluates 12 neuropsychiatric symptoms. The total score on each item can range from 1 to 12 and is obtained by multiplying severity ( 1 "mild" to 3 "severe") by frequency (1 "sometimes" to 4 "very often"). In a previous study principal component analysis of the NPI identified three behavioural sub-syndromes: (a) a hyperactivity factor, including the symptoms disinhibition, irritability, agitation, euphoria, and aberrant motor behaviour (5 items, Cronbach's alpha $=0.73$ ); (b) a mood/apathy factor including depression, apathy, nighttime behavioural disturbances and eating abnormalities ( 4 items, $\alpha=0.63$ ); and (c) a psychosis factor, including hallucinations and delusions ( 2 items, $\alpha=0.72$ ) (Aalten et al. 2003). Total scores for each sub syndrome were computed as the sum of observed NPI item scores for each factor. These three factors were used in this study.

The severity of dementia was rated with the Global Deterioration Scale (GDS; Reisberg et al. 1982). Patient's level of interference with regard to daily activities was measured with the Interview for Deterioration in Daily living activities in Dementia (IDDD; Teunisse and Derix 1991). This questionnaire consists of 20 items reflecting initiative to and actual performance of daily activities. We used a summed score for items concerning actual performance of activities (range 0 to 44) and for items concerning initiative to activities (range 0 to 36). The Mini Mental Status Examination (MMSE; Folstein et al. 1975) was used to measure patient's cognitive functioning. 


\section{Neuropsychological measures}

Cognitive functioning of caregivers and controls was assessed by means of tasks measuring verbal memory, speed of information processing, and cognitive flexibility. Global cognitive functioning was assessed with the Mini Mental State Examination (MMSE; Folstein et al. 1975). The delayed recall score of the Auditory Verbal Learning Test (AVLT; Brand and Jolles 1985) was used to measure memory retrieval. Speed of information processing was assessed with the Letter Digit Coding Test (LDCT), which is a modified version of the Symbol Digit Modalities Test (Smith 1968). Cognitive flexibility was assessed with the Stroop Colour-Word Test (SCWT; Houx et al. 2002; Stroop 1935). The test uses three cards displaying colour names (SCWT I), colour patches (SCWT II), and colour names printed in incongruously coloured ink (SCWT III). The dependent variable is the time needed to read card III minus the mean time needed to read card I and II. Cognitive tests have shown to be reliable and sensitive to detect small differences (Houx et al. 2002). A shortened form of the GIT (Luteijn and van der Ploeg 1983) was used to obtain a measure of general intelligence.

\section{Additional caregiver measures}

The Symptom-Checklist 90 (SCL-90) (Arrindell and Ettema 1986) depression subscale and anxiety subscale were used to assess psychological complaints in caregivers and controls. For each of the 12 behavioural symptoms on the NPI, caregivers rated the level of distress they experienced on a scale from 0 (none) to 5 (extreme). NPI-Distress score is the sum of these 12 ratings (range $0-60$ ).

Caregiver's subjective competence was measured with the Short Sense of Competence Questionnaire (SOC; Vernooij-Dassen et al. 1999). This questionnaire consists of 7 items rated on a 5-point scale (1 "agree very strongly" to 5 "disagree very strongly"; range 7-35). These items reflect three domains of caregivers' feelings of being capable of caring for a demented person: (a) satisfaction with the demented person as a recipient of care; (b) satisfaction with one's own performance as a caregiver; and (c) consequences of involvement in care for the personal life of the caregiver. The content validity and construct validity have been reported elsewhere (Vernooij-Dassen et al. 1999). Cronbach's alpha in this study is 0.77 .

Physical health complaints were measured with the physical functioning subscale of the RAND-36 questionnaire (Van der Zee and Sanderman 1993). Ten items are rated on 3point scales ranging from severely impaired to not at all impaired, with higher scores representing less impairment. 


\section{Data analysis}

Univariate comparisons were performed with Student t-test, Chi Square test and MannWhitney $U$ test. Caregiver cognitive performance was converted to standard z-scores using the data from the control group as the reference. In order to explore group differences in cognitive functioning and the mediating effect of psychological functioning, three forced entry regression analyses were performed to predict cognitive functioning (memory, information processing speed, and cognitive flexibility) by age, education, sex (entered at step 1), group (controls vs. caregivers; entered at step 2), depression and anxiety (entered at step 3).

Next, caregiver cognitive performance was examined as predictor of patient behaviour with repeated measures analysis of covariance (ANCOVA). Therefore, caregivers were assigned to 'low performance' and 'high performance' groups, according to a mediansplit on the memory delayed recall score, Stroop interference score, and LDCT score. Differences in patient behaviour between high and low performance groups for the three cognitive domains were analysed using ANCOVA with 'group' (high versus low performance) and sex as between-subjects factor and 'time' (timing of patient behaviour measurement: baseline, 6 months and 12 months follow-up) as within-subject factor; age, education, and dementia severity were covariates. This analysis was repeated to examine caregiver cognitive performance as predictor of caregiver competence. Significance was tested with two-tailed tests, with $\alpha=.05$.

\section{Results}

\section{Cognitive functioning in caregivers and controls}

Caregivers and control subjects did not differ in psychological complaints or IQ score (see table 1). Univariate analysis of group differences in cognitive functioning indicated that caregivers performed significantly worse than the control subjects on all cognitive domains, except for cognitive flexibility (see table 1). 
Table 1. Demographic and psychological characteristics, and cognitive performance in caregivers and controls

\begin{tabular}{|c|c|c|c|c|c|}
\hline & & $\begin{array}{l}\text { Caregivers } \\
\mathrm{n}=54\end{array}$ & $\begin{array}{l}\text { Controls } \\
n=108\end{array}$ & Test value & P value \\
\hline & & Mean (SD) & Mean (SD) & & \\
\hline \multirow[t]{2}{*}{$\operatorname{Sex}(n, \%)$} & Male & $22(40.7 \%)$ & $44(40.7 \%)$ & $\chi^{2}=0.0$ & 1.000 \\
\hline & Female & $32(59.3 \%)$ & $64(59.3 \%)$ & & \\
\hline \multicolumn{2}{|l|}{ Age } & $68.4(8.5)$ & $68.3(8.4)$ & $\mathrm{t}=0.1$ & 0.927 \\
\hline \multicolumn{2}{|c|}{ Level of education ${ }^{1}$} & $1.78(0.8)$ & $1.76(0.8)$ & $t=0.1$ & 0.888 \\
\hline \multicolumn{2}{|c|}{ SCL anxiety } & $15.3(5.2)$ & $15.2(7.3)$ & $Z=-0.2$ & 0.219 \\
\hline \multicolumn{2}{|c|}{ SCL depression } & $27.7(9.7)$ & $26.0(11.9)$ & $Z=-1.5$ & 0.134 \\
\hline \multicolumn{2}{|c|}{ IQ score } & $114.1(13.7)$ & $115.1(11.6)$ & $t=-0.5$ & 0.638 \\
\hline \multicolumn{2}{|l|}{ MMSE } & $27.9(1.6)$ & $28.6(1.7)$ & $Z=-3.1$ & 0.002 \\
\hline \multicolumn{2}{|c|}{ AVLT (delayed recall) } & $8.6(3.3)$ & $10.1(2.9)$ & $t=-2.7$ & 0.006 \\
\hline \multicolumn{2}{|c|}{ Stroop interference (seconds) } & $117.3(49.4)$ & $104.0(35.5)$ & $t=1.9$ & 0.053 \\
\hline \multicolumn{2}{|c|}{ LDCT (number completed) } & $36.6(12.6)$ & $44.5(8.8)$ & $t=-4.1$ & $\leq 0.001$ \\
\hline
\end{tabular}

MMSE = Mini Mental State Examination $\mid$ SCL-90 = Symptom Checklist-90 | MMSE = Mini Mental State Examination AVLT = Auditory Verbal Learning Test $\mid \mathrm{LDCT}=$ Letter Digit Coding Test

1. educational level was compressed from eight to three levels: low (level 1 and 2), medium (level 3 to 5), and high (level 6 to 8)

Figure 1 shows cognitive performance scores converted to standard z-scores for caregivers, using cognitive performance of the controls as a reference.

Psychological complaints were examined as possible mediators of the relationship between group and cognitive performance. In the caregiver group 15 (28\%) subjects scored high on anxiety and 24 (45\%) scored high on depression compared to norm scores, whereas in the control group 29 (27\%) subjects scored high on anxiety and 32 (30\%) subjects scored high on depression. Forced entry regression analyses were performed to predict cognitive functioning by age, education, sex (entered at step 1), group (entered at step 2), depression and anxiety (entered at step 3). Control subjects performed better than caregivers on tasks measuring global cognitive functioning ( $t=2.5$, $p=0.012)$, memory delayed recall $(t=2.9, p=0.004)$, and information processing speed $(t=3.7, p \leq 0.001)$, but not for cognitive flexibility ( $t=-1.8, p=0.083)$. In addition, complaints of depression and anxiety were only predictive for memory performance (respectively $t=2.5, p=0.014$ and $t=-2.9, p=0.004$ ), accounting for $6 \%$ of variance in memory performance with the total model explaining $22 \%(F=6.1 ; p \leq 0.001)$. 


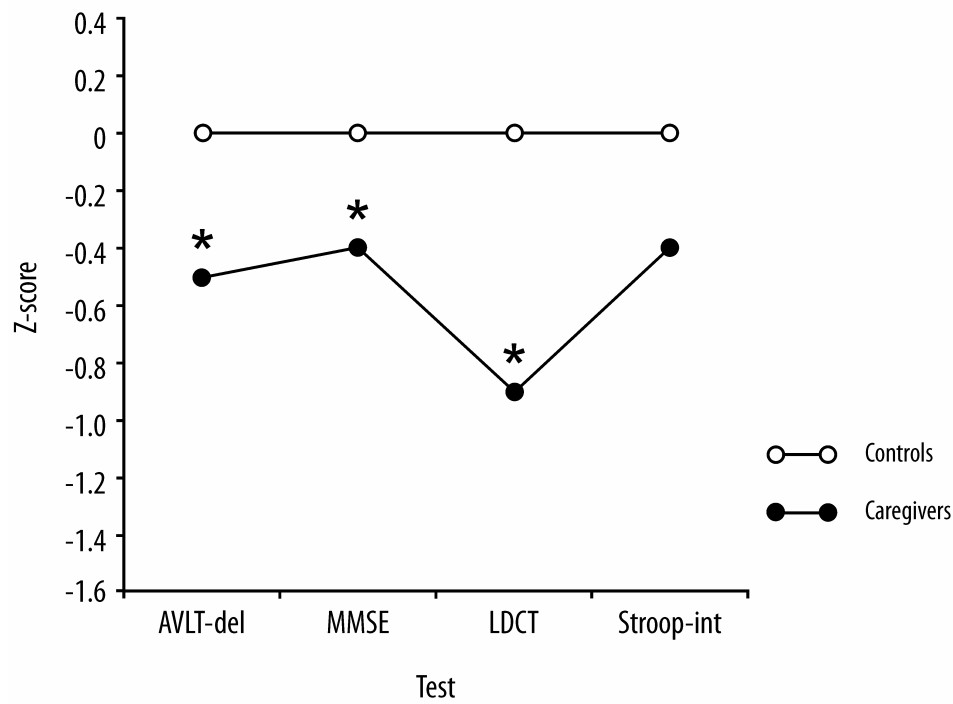

Figure 1. Standardized performance scores for caregivers with respect to controls | $* P<0.05$

\section{Differences between caregiver cognitive performance groups}

Cognitive measures (AVLT, Stroop interference, LDCT) were dichotomised by a median split in a high and low caregiver performance group. Table 2 shows descriptive data of the low and high cognitive performance groups for each cognitive domain. The high cognitive performance group was significantly younger than the low performance group on all cognitive domains (all p-values lower than 0.010). Furthermore, caregivers in the low cognitive speed group were significantly lower educated $(t=-2.7, p=0.009)$ and had more physical health complaints $(Z=-2.8, p=0.006)$ than caregivers in the high cognitive speed group. Also caregivers in the low cognitive flexibility group had more physical health complaints than caregivers in the high cognitive flexibility group $(Z=-2.4, p=$ 0.015). The low performance group on information processing speed tended to use more medication (i.e. antidepressants, antipsychotics or anxiolytics) $\left(\chi^{2}=3.7, p=0.054\right)$. In addition, there were no differences in medication use between the high and low performance groups for memory $\left(\chi^{2}=0.1, p=0.771\right)$ and cognitive flexibility $\left(\chi^{2}=0.8, p=\right.$ 0.386). 
Table 2. Descriptive data for high versus low cognitive performance groups per cognitive domain

\begin{tabular}{|c|c|c|c|c|c|c|c|c|c|}
\hline & \multicolumn{3}{|c|}{$\begin{array}{c}\text { Memory } \\
\text { AVLT delayed recall }\end{array}$} & \multicolumn{3}{|c|}{$\begin{array}{l}\text { Cognitive flexibility } \\
\text { Stroop interference }\end{array}$} & \multicolumn{3}{|c|}{$\begin{array}{l}\text { Speed } \\
\text { LDCT }\end{array}$} \\
\hline & $\begin{array}{l}\text { Low } \\
n=24\end{array}$ & $\begin{array}{l}\text { High } \\
n=30\end{array}$ & $\begin{array}{l}\mathrm{P} \\
\text { value }\end{array}$ & $\begin{array}{l}\text { Low } \\
n=27\end{array}$ & $\begin{array}{l}\text { High } \\
n=27\end{array}$ & $\begin{array}{l}P \\
\text { value }\end{array}$ & $\begin{array}{l}\text { Low } \\
\mathrm{n}=25\end{array}$ & $\begin{array}{l}\text { High } \\
n=29\end{array}$ & $P$ value \\
\hline \multicolumn{10}{|l|}{ Caregiver } \\
\hline Male/Female' & $13 / 11$ & $9 / 21$ & .097 & $14 / 23$ & $8 / 19$ & .166 & $8 / 17$ & $14 / 15$ & .274 \\
\hline $\mathrm{Age}^{2}$ & $71.7(7.5)$ & $65,8(8.4)$ & .010 & $72.7(7.9)$ & $64.2(6.7)$ & $\leq .001$ & $72.2(7.3)$ & $65.3(8.2)$ & .002 \\
\hline Education & $1.8(0.8)$ & $1.8(0.8)$ & .910 & $1.8(0.8)$ & $1.7(0.8)$ & .735 & $1.5(0.8)$ & $2.0(0.7)$ & .009 \\
\hline $\begin{array}{l}\text { Contact } \\
\text { hours }\end{array}$ & $\begin{array}{l}153.1 \\
(15.4)\end{array}$ & $\begin{array}{l}154.0 \\
(13.3)\end{array}$ & .828 & $\begin{array}{l}154.4 \\
(13.7)\end{array}$ & $\begin{array}{l}152.9 \\
(14.8)\end{array}$ & .701 & $\begin{array}{l}153.2 \\
(15.7)\end{array}$ & $\begin{array}{l}153.9 \\
(12.9)\end{array}$ & .856 \\
\hline $\begin{array}{l}\text { Care } \\
\text { (months) }\end{array}$ & $\begin{array}{l}27.2 \\
(25.4)\end{array}$ & $\begin{array}{l}28.4 \\
(25.9)\end{array}$ & .863 & $\begin{array}{l}22.9 \\
(20.1)\end{array}$ & $\begin{array}{l}32.9 \\
(29.5)\end{array}$ & .151 & $\begin{array}{l}28.4 \\
(24.8)\end{array}$ & $\begin{array}{l}27.4 \\
(26.5)\end{array}$ & .884 \\
\hline SOC & $25.7(5.5)$ & $25.3(6.2)$ & .806 & $26.2(4.8)$ & $24.7(6.7)$ & .382 & $24.6(6.8)$ & $26.2(4.9)$ & .307 \\
\hline NPI distress & $10.0(7.2)$ & $9.2(9.5)$ & .722 & $9.2(8.5)$ & $10.0(8.7)$ & .717 & $10.8(9.3)$ & $8.6(7.9)$ & .347 \\
\hline MADRS & $7.6(6.1)$ & $7.9(5.9)$ & .837 & $7.5(4.7)$ & $8.0(7.1)$ & .735 & $9.2(6.3)$ & $6.5(5.4)$ & .074 \\
\hline RAND phys & $23.8(5.5)$ & $25.1(4.2)$ & .499 & $22.6(5.5)$ & $26.2(3.3)$ & .015 & $22.2(5.7)$ & $26.5(2.5)$ & .006 \\
\hline SCL depr & $\begin{array}{l}25.4 \\
(8.2)\end{array}$ & $\begin{array}{l}29.0 \\
(10.4)\end{array}$ & .241 & $\begin{array}{l}28.5 \\
(9.4)\end{array}$ & $\begin{array}{l}27.1 \\
(10.0)\end{array}$ & .640 & $\begin{array}{l}30.3 \\
(11.4)\end{array}$ & $\begin{array}{l}25.9 \\
(8.1)\end{array}$ & .132 \\
\hline \multicolumn{10}{|l|}{ Patient } \\
\hline MMSE & $18.2(5.2)$ & $18.7(5.5)$ & .734 & $18.3(5.2)$ & $18.6(5.5)$ & .840 & $18.7(5.5)$ & $18.2(5.2)$ & .727 \\
\hline IDDD & $\begin{array}{l}20.4 \\
(10.9)\end{array}$ & $\begin{array}{l}19.0 \\
(12.5)\end{array}$ & .673 & $\begin{array}{l}18.3 \\
(11.8)\end{array}$ & $\begin{array}{l}20.9 \\
(11.7)\end{array}$ & .415 & $\begin{array}{l}17.1 \\
(11.9)\end{array}$ & $\begin{array}{l}21.8 \\
(11.4)\end{array}$ & .142 \\
\hline NPI & $\begin{array}{l}17.5 \\
(14.3)\end{array}$ & $\begin{array}{l}17.5 \\
(20.0)\end{array}$ & .348 & $\begin{array}{l}16.8 \\
(19.5)\end{array}$ & $\begin{array}{l}18.2 \\
(15.7)\end{array}$ & .746 & $\begin{array}{l}20.4 \\
(19.5)\end{array}$ & $\begin{array}{l}14.9 \\
(15.6)\end{array}$ & .314 \\
\hline
\end{tabular}

SOC = Sense of Competence Questionnaire | NPI = Neuropsychiatric Inventory | MADRS = Montgomery Asberg Rating Scale $\mid$ RAND-phys = RAND physical subscale $\mid \mathrm{SCL}$ depr $=$ Symptom Checklist-90 depression subscale | MMSE = Mini Mental State Examination | IDDD = Interview of Daily living activities in Dementia

1. values represent number of males and females $\mid 2$. educational level was compressed from eight to three levels: low (level 1 and 2), medium (level 3 to 5), and high (level 6 to 8) 


\section{Caregiver cognitive functioning as predictor of patient behaviour}

ANCOVA was performed to investigate differences in patient behaviour between the high and low cognitive performance groups, adjusted for age, sex, education and dementia severity. Results showed no significant overall difference in general levels of behavioural symptoms (total NPI) between the groups for memory $(F=2.6(1,32), p=0.115)$, cognitive flexibility $(F=0.4(1,32), P=0.539)$, or cognitive speed $\left(F=0.1_{(1,32),} P=0.777\right)$. There was a near significant time by memory performance interaction effect $(F=2.9(2,64), p=0.065)$, with an increase in patient behavioural problems in the low memory performance group in the first 6 months follow-up (figure 2). Therefore, an additional ANCOVA was performed to test differences in change between both memory performance groups in the first 6 months follow-up. Indeed, this analysis showed a significant increase in the low performance group in comparison with the high performance group $\left(F=5.1_{(1,32),} p=\right.$ 0.030). Thus, lower caregiver memory performance was related to an increase in patient behavioural problems.

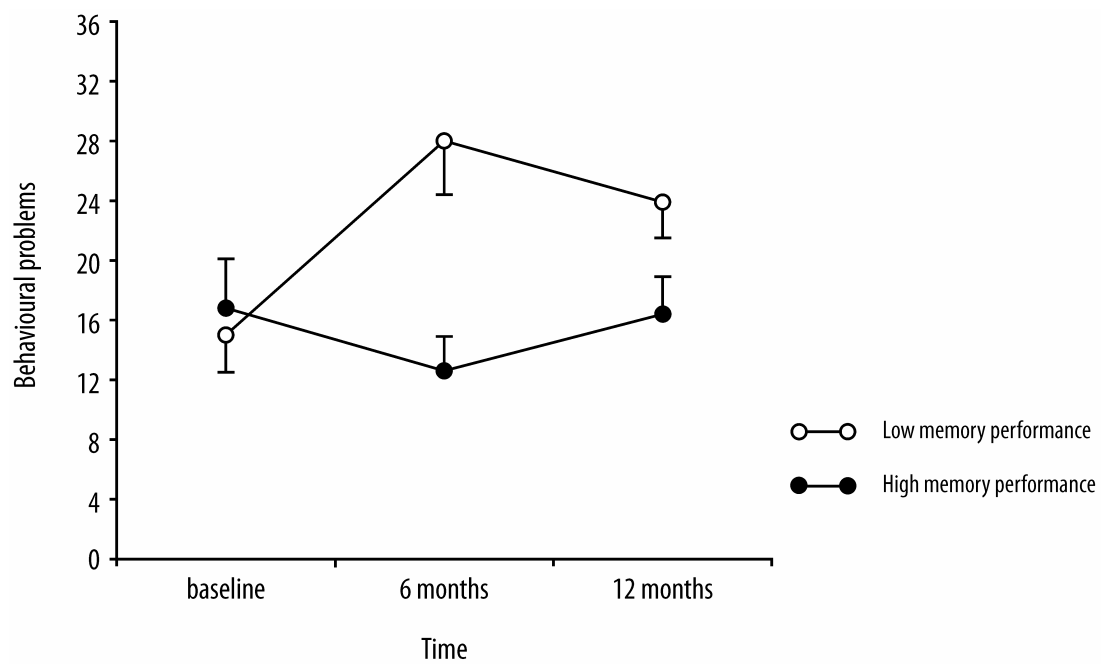

Figure 2. Behavioral problems (mean \pm SEM) in patients of caregivers with low ( $n=15)$ and high memory performance $(n=23)$ at baseline, 6 months and 12 months follow-up

To examine differences in specific types of patient behaviours between the high and low memory performance group, ANCOVA was repeated for the three behavioural subsyndromes (mood/apathy, hyperactivity, and psychosis). Results showed no overall group differences for memory performance in patient hyperactivity $(F=1.1(1,32), p=0.302)$, 
psychosis $(F=0.4(1,32), p=0.520)$, or mood/apathy $(F=3.7(1,32), p=0.064)$. However, there was a significant difference in change in hyperactivity over time $(F=4.7(2,64), p=0.021)$. Again, there was an increase in patient hyperactivity in the low memory performance group of caregivers, in the first 6 months of follow-up. To investigate whether the impact of caregiver memory performance on patient hyperactivity was mediated by caregiver distress, an additional ANCOVA was performed with caregiver memory performance as predictor of patient hyperactivity, adjusted for caregiver distress (NPI-D). This analyses showed that the time by memory performance interaction effect $(F=4.9(2,62), p=0.018)$ remained significant after adjusting for caregiver distress $(F=27.4(2,31), p \leq 0.001)$.

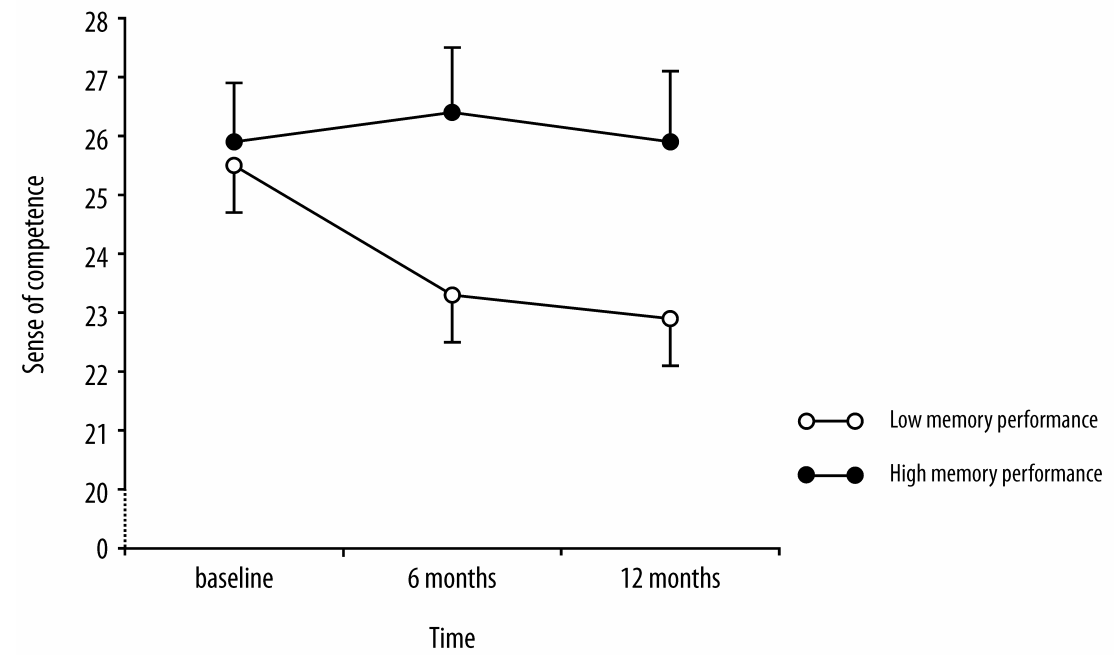

Figure 3. Sense of competence (mean \pm SEM) in caregivers with low $(n=13)$ and high memory performance $(n=22)$ at baseline, 6 months and 12 months follow-up

\section{Caregiver cognitive functioning as predictor of caregiver competence}

In addition, we hypothesised that patients of caregivers with lower levels of cognitive functioning might feel less competent than patients of caregivers with higher levels of cognitive functioning. Therefore, ANCOVA was performed to investigate differences in caregiver competence between the high and low cognitive performance groups, adjusted for age, sex, education, and dementia severity. Results showed no significant overall difference in feelings of competence between the groups for memory $(F=1.5(1,29)$, $p=0.228)$, cognitive flexibility $\left(F=0.03_{(1,29),} p=0.863\right)$, or cognitive speed $\left(F=0.001_{(1,29),} p=\right.$ 
0.974). There were also no significant interaction effects between cognitive performance and feelings of competence, although a decrease in feelings of competence was shown between baseline and 6 months follow-up in the low memory performance group (see Figure 3). To test this difference in change between both groups an additional ANCOVA was performed for baseline and 6 months follow-up. Indeed, this analysis showed that the low memory performance group had a significant decrease in feelings of competence when compared to the high memory performance group from baseline to 6 months follow up $(F=6.3(1,39), p=0.016)$.

\section{Discussion}

The results show that spousal caregivers of dementia patients have on average lower levels of cognitive functioning than age-, sex-, and education- matched controls. Caregivers performed significantly worse on measures of general cognitive functioning, speed of information processing, and verbal memory. In general, caregiver cognitive functioning was sub-optimal rather than impaired. Furthermore, low performance on verbal memory was related to a decrease in caregiver competence, and an increase in patient behavioural symptoms, in particular hyperactivity symptoms.

Interestingly, Caswell and colleagues (2003) recently obtained evidence which is compatible with our findings. They found lower levels of complex attention and speed of information processing in caregivers compared to controls, after adjustment of age differences. Cognitive suboptimal functioning in caregivers can probably be explained by chronic stress levels due to the caregiving situation. Behavioural problems lead to chronic stress which in turn can elevate levels of cortisol in caregivers of dementia patients (Vugt de et al. 2004, in press). High levels of cortisol have been widely associated with impaired cognitive functioning, particularly with reduced cognitive attention and memory (Lupien et al. 1994; Lupien et al. 1998; McEwen and Sapolsky 1995; Sapolsky et al. 1986). Data on psychological complaints were available in the control group and used as a proximate measure of chronic stress to investigate the mediating effect of psychological functioning in the association between group and cognitive performance. However, differences in cognitive functioning between the groups were not associated to psychological complaints, and may be better explained by more specific measures of stress. Furthermore, group differences in cognitive functioning may be related to differences in physical health problems and related medication use. The control group consisted of healthy subjects without medical conditions or psychotropic drug use that could interfere with normal cognitive function. In contrast, caregivers of dementia patients are known to 
perceive their health to be worse than non-caregivers, to use more psychotropic medication and to visit their physician more often (Baumgarten et al. 1992; Katon et al. 1982; Kiecolt-Glaser et al. 1991), which may reduce cognitive functioning. Indeed, caregivers in the low speed and cognitive flexibility performance groups reported somewhat more health complaints than caregivers in the high performance groups. In addition, caregivers in the low cognitive speed group tended to use more psychotropic medication.

Potential effects of suboptimal caregiver cognitive functioning on patient outcomes were assessed longitudinally. Low performance on a measure of verbal memory was related to an increase in patient behavioural symptoms, in particular hyperactivity symptoms. This finding is in line with previous results from MAASBED (Vugt et al. 2004), that caregivers who use less effective care strategies increase the risk of hyperactive behaviour in the patient. Effective care strategies may greatly depend on the cognitive abilities of the caregiver, in order to meet the needs and shortcomings of the patient. Given the complex and changing demands in the caregiving situation, suboptimal cognitive functioning may affect daily caregiver functioning, even without a clear cognitive impairment (Caswell et al. 2003). Therefore, cognitively "impaired" caregivers may be less able to develop adequate care strategies, resulting in increased hyperactive behaviour in the patient (Vugt et al. 2004)

In addition, low memory performance in caregivers was associated with a decrease in feelings of competence in caregivers. Again, this can be explained by the possible influence of cognitive abilities on care strategies. Previous results from MAASBED (Vugt et al. 2004) indicated that caregivers who use a non-adapting strategy feel less competent than caregivers who use other care strategies. Furthermore, poorer cognitive functioning may be disconcerting to caregivers and cause lower feelings of competence, as they may even worry about getting dementia themselves. This may also explain why only memory performance was associated with lower feelings of competence, as memory problems are the most salient characteristic of dementia and therefore may cause the most uneasiness in caregivers about their own mental health.

There were several limitations to this study. First, lowered cognitive functioning in caregivers may have biased the reports of patient problems, since part of patient information was only obtained by caregiver judgement. However, the fact that reduced memory performance was specifically related to higher levels of patient hyperactivity and 
not to other problems makes it unlikely that a bias in caregiver reports can explain this finding.

Second, there probably exists a bi-directional relationship between caregiver competence, patient behaviour and caregiver cognitive functioning. High levels of patient problems will cause caregiver stress, which in turn is associated with impaired cognitive functioning. However, the finding that patient behavioural problems increased in the low memory performance group, while at baseline no significant differences existed between the groups in patient behaviour suggests temporal precedence and supports the view that reduced cognitive functioning is also a predictor of patient behaviour and caregiver competence.

Our findings indicate that spousal caregivers of dementia patients show reduced levels of cognitive functioning compared to healthy matched controls. Furthermore, sub-optimal cognitive functioning in caregivers has profound implications for patient as well as caregiver outcomes. Low memory performance was associated with a reduction in caregiver's feelings of competence and an increase in patient behavioural symptoms, especially hyperactivity. These results imply that one should be alert to cognitive problems in older caregivers. Even minor memory problems in caregivers may affect their ability to provide adequate care and the quality of life of both patient and caregiver.

\section{Key points}

- Spousal caregivers show suboptimal cognitive functioning compared to healthy controls

- $\quad$ Reduced caregiver cognitive functioning is associated with a decrease in feelings of caregiver competence and an increase in levels of patient hyperactivity 
Chapter 10. Consequences of cognitive functioning in the spousal caregivers of dementia patients 


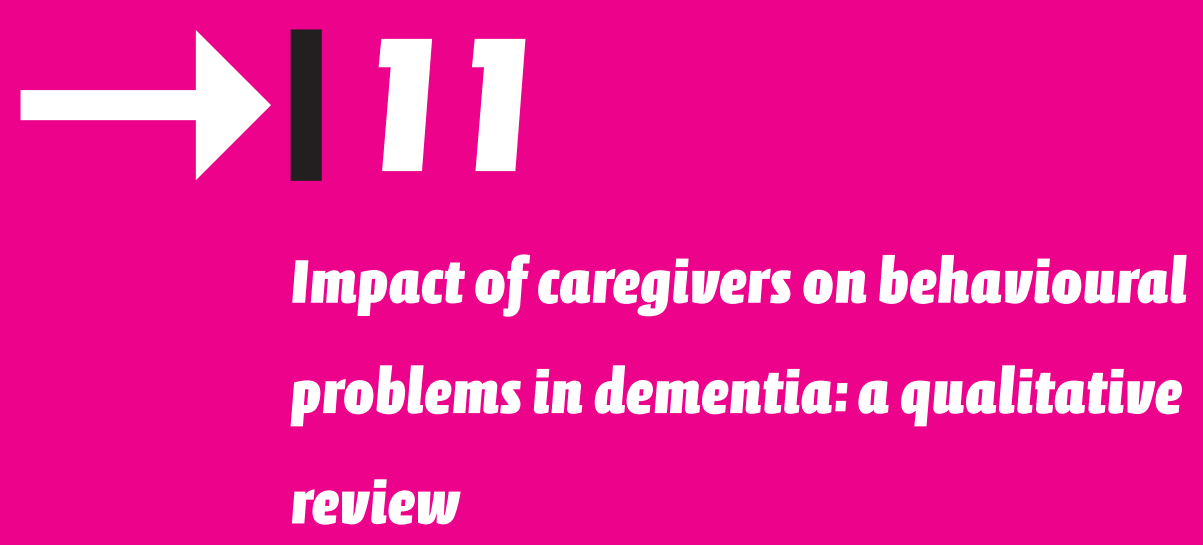




\section{Abstract}

Objective: This paper provides a review of research findings published between 1966 and May 2003 concerning the influence of informal caregivers on behavioural problems in dementia.

Methods: MEDLINE, Psychinfo, EMBASE, and the Cochrane databases were searched to identify research articles on informal caregivers, dementia and behavioural problems. Studies had to involve an intervention targeting informal caregivers and provide information on patient behavioural problems before and after the intervention period. The methodological quality of the studies was assessed according to standardized criteria. Studies with an adequate methodological quality were included in the review.

Results: Twenty-six papers met criteria for inclusion. Two sets of two duplicate papers were identified and the two publications with the lowest methodological rating were excluded. Of the remaining 24 papers there were 13 papers rated as methodological adequate. Methodological limitations included small group sizes, inadequate descriptions of the target group, lack of comparability between groups, imprecise data collection methods, and a lack of adjustment for co-interventions. Notwithstanding these limitations, caregiver interventions, in particular behavioural management training, show beneficial effects for patient behavioural problems.

Conclusions: Caregiver interventions are potentially successful in reducing patient behavioural problems. Methodological improvement and refinement of intervention studies is needed. Tailoring interventions to patient and caregiver characteristics will maximize successfulness. 


\section{Introduction}

Behavioural and Psychological symptoms of dementia (BPSD) can be defined as a heterogeneous range of psychological reactions, psychiatric symptoms and behaviours resulting from the presence of dementia. Problems such as depression, apathy, agitation, aggression, hallucinations and delusions are present in most patients at some point during the disease course (Aalten et al. 2003; Cummings 1990). They are important determinants of caregiver burden (Donaldson et al. 1998; Draper et al. 1995; Vugt et al. 2003) and they increase the risk of institutionalisation (Hope et al. 1998; O'Donell et al. 1992).

These behavioural symptoms are produced by a complex interaction between several factors. Much of the literature has focused on biological (neurochemical and neuropathological) (Forstl 2000) and psychological (e.g. premorbid personality) (Meins et al. 1998; Strauss 1997) predictors of BPSD. However, environmental aspects such as caregiver functioning could contribute to the etiology of BPSD as well. Theoretical support for this association can be found in Lawton's ecological model (Lawton 1975), which suggests that persons with dementia are even more likely than average persons to be vulnerable to the impact of their environment because of their lowered competence and function. Consequently, a high demand from the environment can result in inappropriate behaviour of the patient (Eriksson 2000). The literature relating to the influence of caregiver functioning on the manifestation of behavioural problems in dementia is full of anecdotal reports and recommendations (Marchello et al. 1995; Stewart 1995; Teri et al. 2002). However, most of the caregiving research has focused on caregiver consequences of patient behavioural problems (Donaldson et al. 1997; Gaugler et al. 2000a; Hooker et al. 2002; Pruchno and Resch 1989). Only few studies have addressed the issue whether caregiver characteristics predict such problems in the patient. In the MAAstricht Study of Behaviour in Dementia (MAASBED) we found that caregiver management strategies were related to levels of patient hyperactivity (Vugt et al. 2004). Vitaliano et al. (1993) found that ratings of a caregiver's expressed emotion were predictive of increased behavioural problems over time. In addition, caregiver distress has been found to be predictive for feeding difficulties (Riviere et al. 2002) and delusions (Riello et al. 2002). A study of Hamel et al. (1990) showed that a troubled premorbid relationship between patient and caregiver was a significant predictor of patient aggression. Roberto et al. (1998) found that patients of caregivers with dysfunctional communication patterns exhibited more behavioural problems. 
Taken together, results from these few studies suggest that several aspects of caregiver functioning have an impact on patient behaviour. Modifying the caregiver's approach towards the patient's behaviour may lead to a reduction of the latter. Although there is still much unknown about this issue, numerous caregiver training programmes are based on the assumption that caregiver functioning influences patient outcomes. Many interventions are aimed at learning caregivers skills to reduce patient behavioural problems. Surprisingly, in the caregiver intervention literature more emphasis has been placed on caregiver outcomes (such as burden or levels of depression) than on patient functioning (Schulz et al. 2002). Clarification of the effectiveness of these training programmes to reduce patient problems is needed.

The purpose of this paper is to provide a review of relevant research findings concerning the issue whether caregiver functioning influences the manifestation of behavioural problems in dementia. Given the small amount of studies assessing caregiver characteristics as predictors of patient behavioural problems, we focused on the question if patient behavioural problems improve after caregiver interventions.

\section{Methods}

\section{Literature search}

Data collection strategies included (i) searches of MEDLINE (1966- May 2003), Psychinfo (1966- May 2003), EMBASE (1984- May 2003) and the Cochrane databases (issue 2, 2003) (ii) searches of references listed in the reviewed papers and meta-analyses. Key words were formulated according to the PICO-method (Patient population, Intervention, Control/Comparison, Outcome). The following key words were used: dementia/ Alzheimer('s)/ vascular/ Lewy body/ Frontotemporal/ Parkinson/ mixed; caregiver(s)/ carer(s)/ family/ spouse(s)/ child(ren)/ relative(s)/ psychosocial/ non-pharmalogical; controlled trial/ placebo/ case study; BPSD/ behaviour/ behaviour/ psychosis/ delusions/ paranoia/ depression/ aggression/ agitation/ restlessness/ anxiety/verbal/ kicking/ biting/ spitting/yelling/wandering/ repetitive/ pacing/sundowning/ shouting/ hitting.

The papers to be selected for this review had at least to meet the following criteria: (i) patients had to be diagnosed with dementia; (ii) the study had to include informal (nonprofessional) caregivers; (iii) the study had to involve an intervention targeting informal caregivers; (iv) there were comparisons of patient behavioural problems before and after 
the intervention period (v) there was a full report available (vi) for practical reasons, the study had to be written in English, Dutch, French or German.

All titles and abstracts were screened and possibly relevant articles were obtained and assessed for inclusion. Twenty-six articles met all inclusion criteria.

\section{Study quality assessment}

The methodological quality of the included studies was assessed according to standardized methodological criteria (de Vet et al. 1997). A list of 47 criteria was designed at forehand and concerned 11 methodological aspects (table 1), based on the method developed by de Vet and colleagues (1997). The criteria concerned three aspects of the study: the internal validity (e.g. randomization, blinding, loss to follow up); the precision of the study (e.g. sample size and variability); the relevance of choices with respect to the study population, interventions and outcome parameters. Maximum scores per criterion were determined beforehand, with more important criteria receiving relatively higher maximum scores. A total maximum score of 100 could be obtained per study, with an arbitrary score higher than 55 regarded as representing an adequate quality of the paper. Two independent raters (NR, MM) scored all papers on the 11 methodological aspects. In order to prevent review bias, all papers were blinded by the first author (MdV). Information was deleted about authors, name and location of institute, journal and publication year. The two raters agreed on the score for 195 of the 275 aspects (11 aspects and 25 papers; 71\%). A consensus meeting was held to identify and discuss disagreements between the raters. All disagreements were solved during this meeting. A total score was obtained per article, resulting in a hierarchical list in which higher scores indicated a higher methodological quality of the paper. Methodological ratings were based solely on material presented in the publication, therefore studies may have been under-rated if papers failed to mention important details of the study.

\section{Results}

\section{Methodological rating}

Twenty-six papers met the inclusion criteria. Two sets of two duplicate papers (Bourgeois et al. 1997; Bourgeois et al. 2002; Teri et al. 2000; Teri et al. 1998) were based on the same data; of these the two studies with the lowest scores were excluded (Bourgeois et al. 1997; Teri et al. 1998). Thus, twenty-four publications were included in this review (table 1). The mean methodological quality was adequate (mean score $=58$ ). Scores ranged between 
22 and 83, with 13 papers (54\%) scoring above the cut-off of 55 (adequate quality) (Bourgeois et al. 2002; Gendron et al. 1996; Gerdner et al. 1996; Gitlin et al. 2001; Gormley et al. 2001; Hebert et al. 1994; Hebert et al. 2003; Huang et al. 2003; Marriott et al. 2000; Ostwald et al. 1999; Quayhagen et al. 2000; Teri et al. 2000; Teri et al. 1997)

Table 1. Methodological quality of included studies

\begin{tabular}{|c|c|c|c|c|c|c|c|c|c|c|c|c|c|}
\hline Criterion & 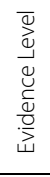 & ¿ & के & U & ڤ̀ & 亡 & ப் & ¿ & 主 & $=$ & $=$ & 立 & 쥼 \\
\hline $\begin{array}{l}\text { Maximum score } \\
\text { Included studies: }\end{array}$ & & 10 & 10 & 10 & 6 & 8 & 10 & 11 & 7 & 12 & 8 & 8 & 100 \\
\hline 1. Gormley 2001 & A & 7 & 10 & 10 & 4 & 8 & 9 & 8 & 7 & 10 & 4 & 6 & 83 \\
\hline 2. Marriot 2000 & A & 7 & 10 & 10 & 4 & 5 & 8 & 8 & 7 & 11 & 6 & 6 & 82 \\
\hline 3. Teri 2000 & A & 9 & 8 & 10 & 4 & 3 & 8 & 8 & 7 & 12 & 5 & 8 & 82 \\
\hline 4.Gerdner 2002 & A & 7 & 10 & 10 & 4 & 3 & 10 & 10 & 0 & 11 & 8 & 6 & 79 \\
\hline 5.Hebert 2003 & A & 4 & 10 & 10 & 5 & 4 & 10 & 5 & 7 & 11 & 5 & 6 & 77 \\
\hline 6.Gitlin 2001 & A & 9 & 10 & 8 & 4 & 6 & 10 & 4 & 7 & 4 & 3 & 6 & 71 \\
\hline 7.Gendron 1996 & A & 5 & 10 & 2 & 2 & 8 & 9 & 8 & 0 & 10 & 8 & 7 & 69 \\
\hline 8.Hebert 1994 & A & 8 & 10 & 2 & 4 & 3 & 9 & 4 & 0 & 10 & 8 & 7 & 65 \\
\hline 9.Teri 1997 & A & 7 & 10 & 0 & 4 & 4 & 9 & 8 & 0 & 11 & 4 & 6 & 63 \\
\hline 10.Bourgeois 2002 & A & 9 & 6 & 2 & 4 & 4 & 8 & 8 & 0 & 10 & 8 & 4 & 63 \\
\hline 11. Ostwald 1999 & A & 6 & 10 & 2 & 3 & 3 & 10 & 5 & 0 & 6 & 6 & 6 & 57 \\
\hline 12. Huang 2003 & A & 6 & 10 & 2 & 5 & 4 & 9 & 6 & 0 & 7 & 4 & 4 & 57 \\
\hline 13.Quahagen 2000 & A & 8 & 8 & 2 & 2 & 0 & 9 & 4 & 0 & 12 & 5 & 6 & 56 \\
\hline 14. Haupt 2000 & $C$ & 5 & 0 & 2 & 0 & 8 & 10 & 0 & 7 & 6 & 4 & 7 & 55 \\
\hline 15.Zarit 1987 & B & 9 & 10 & 2 & 0 & 3 & 7 & 5 & 0 & 5 & 8 & 6 & 55 \\
\hline 16.Hinchliffe 1995 & B & 8 & 10 & 2 & 1 & 3 & 9 & 6 & 0 & 7 & 7 & 2 & 55 \\
\hline $\begin{array}{l}\text { 17.Quayhagen } \\
1995\end{array}$ & B & 3 & 10 & 2 & 0 & 4 & 8 & 6 & 0 & 8 & 8 & 6 & 55 \\
\hline 18.Chang 1999 & B & 4 & 10 & 2 & 4 & 2 & 7 & 7 & 0 & 5 & 4 & 4 & 49 \\
\hline 19.Kuhn 2001 & B & 6 & 0 & 6 & 0 & 3 & 9 & 0 & 0 & 5 & 7 & 6 & 42 \\
\hline 20.Zannetti 1998 & B & 6 & 0 & 0 & 4 & 4 & 8 & 8 & 0 & 3 & 5 & 3 & 42 \\
\hline 21. Done 2001 & B & 5 & 10 & 2 & 0 & 2 & 9 & 4 & 0 & 4 & 1 & 4 & 41 \\
\hline 22. Burgener 1998 & B & 2 & 10 & 0 & 3 & 2 & 5 & 5 & 0 & 5 & 4 & 1 & 37 \\
\hline 23. Teri 1991 & C & 5 & 0 & 0 & 0 & 8 & 6 & 0 & 0 & 2 & 5 & 3 & 29 \\
\hline 24. Bianchetti 1997 & B & 3 & 0 & 0 & 0 & 0 & 7 & 2 & 0 & 4 & 6 & 0 & 22 \\
\hline Mean & & 7 & 8 & 4 & 3 & 4 & 8 & 5 & 2 & 8 & 6 & 5 & 58 \\
\hline$\%$ of max score & & 70 & 80 & 40 & 50 & 50 & 80 & 45 & 28 & 58 & 63 & 63 & 58 \\
\hline
\end{tabular}

Evidence level: $\mathrm{A}=$ randomised clinical trial, high quality $\mid \mathrm{B}=$ randomised clinical trial, moderate quality $\mid \mathrm{C}=$ nonrandomised trial $\mid A^{\prime}=$ selection and restriction $\mid B^{\prime}=$ treatment allocation ||$C^{\prime}=$ study size $\mid D^{\prime}=$ prognostic comparability of groups $\mid \mathrm{E}^{\prime}=$ loss to follow up $\mid \mathrm{F}^{\prime}=$ intervention/experimental $\mid \mathrm{G}^{\prime}=$ intervention/control $\mid \mathrm{H}^{\prime}=$ extra treatments $\mid I^{\prime}=$ outcome measures $\mid J^{\prime}=$ follow up period $\mid K^{\prime}=$ data analysis and presentation. 
Eight papers (33\%) gave only a very brief description of the study population (Bianchetti et al. 1997; Burgener et al. 1998; Chang 1999; Done and Thomas 2001; Haupt et al. 2000; Kuhn and Mendes de Leon 2001; Teri and Uomoto 1991; Zannetti et al. 1998), with most studies not reporting on caregiver kinship or contact intensity with the patient. Most studies were randomized controlled trials, only five studies were non-randomized (21\%) (Bianchetti et al. 1997; Haupt et al. 2000; Kuhn and Mendes de Leon 2001; Teri and Uomoto 1991; Zanetti et al. 1998). Study size was based on a power analysis in five studies (21\%) (Gerdner et al. 2002; Gormley et al. 2001; Hebert et al. 2003; Marriott et al. 2000; Teri et al. 2000), of the remaining papers only two studies had a group size of fifty or more (Gitlin et al. 2002; Kuhn and Mendes de Leon 2001). Prognostic comparability between the intervention and control groups was not, or poorly, described in ten papers (42\%), with fifteen studies (63\%) not reporting on comparability of patient behavioural problems at baseline. To assess patient behavioural problems, sixteen different measures were used with twelve studies (50\%) using the original or revised Memory and Behaviour Problems Checklist (table 2). In seven studies (29\%) there was no loss to follow-up (Bianchetti et al. 1997; Gendron et al. 1996; Gormley et al. 2001; Hair et al. 1988; Haupt et al. 2000; Marriott et al. 2000; Teri and Uomoto 1991); eleven of the remaining papers reported reasons for dropping out. Most studies gave an adequate description of the intervention and control condition, however in most studies both conditions lacked comparability. In six studies (25\%) more than one intervention group was used besides a control intervention (Bourgeois et al. 2002; Burgener et al. 1998; Gitlin et al. 2001; Quayhagen et al. 2000; Teri et al. 2000; Zarit et al. 1987). Only six studies (25\%) reported on comparability or avoidance of co-interventions such as patient medication. Four studies (17\%) reported no follow-up measurement (Gormley et al. 2001; Haupt et al. 2000; Quayhagen et al. 2000; Teri et al. 2000). A follow-up period of 1 to 3 months was reported in seven papers (29\%), 3 to 6 months in four studies (17\%), and 6 months or longer in nine studies (38\%). Only five studies performed an intention-to-treat analysis (21\%) (Gendron et al. 1996; Gerdner et al. 2002; Haupt et al. 2000; Hebert et al. 1994; Teri et al. 2000). 
Table 2. Characteristics of included studies

\begin{tabular}{|c|c|c|c|c|c|}
\hline Study & $\begin{array}{l}\text { Intervention } \\
\text { (group size at } \\
\text { entry) }\end{array}$ & Control & $\begin{array}{l}\text { Design/ } \\
\text { duration }\end{array}$ & Scale & Outcome \\
\hline \multicolumn{6}{|c|}{ Behavioural management training } \\
\hline Gormley (2001) & $\begin{array}{l}\text { Behavioural } \\
\text { management of } \\
\text { aggression (IGr, 34; } \\
\text { CGr 28) }\end{array}$ & Yes & $\begin{array}{l}\text { RCT; } \\
8 \text { weeks }\end{array}$ & $\begin{array}{l}\text { Behave-AD } \\
\text { RAGE }\end{array}$ & $\begin{array}{l}\text { Trend towards } \\
\text { reduction in } \\
\text { aggression in } \\
\text { treatment group }\end{array}$ \\
\hline Marriot (2000) & $\begin{array}{l}\text { Cognitive- } \\
\text { behavioural } \\
\text { intervention (IGr, } \\
14 ; 2 \text { CGr of 14) }\end{array}$ & Yes & $\begin{array}{l}\text { RCT; } \\
9 \text { months } \\
3 \text { months FU }\end{array}$ & $\begin{array}{l}\text { CSDD } \\
\text { MOUSEPAD }\end{array}$ & $\begin{array}{l}\text { Reduction in } \\
\text { behaviour post } \\
\text { treatment (not at } \\
\text { FU) }\end{array}$ \\
\hline Teri (2000) & $\begin{array}{l}\text { Behavioural } \\
\text { Management of } \\
\text { agitation versus } \\
\text { haloperidol/ } \\
\text { trazodone (BMT, } \\
41 ; \text { Hal, 34; Traz, } \\
\text { 37; PL, 36) }\end{array}$ & Placebo & $\begin{array}{l}\text { RCT; } \\
16 \text { weeks 3, 6, } \\
12 \text { months } \\
\text { FU }\end{array}$ & $\begin{array}{l}\text { BRSD; } \\
\text { RMBPC; } \\
\text { CMAl; ABID }\end{array}$ & $\begin{array}{l}32 \% \text { improved in } \\
\text { BMT versus } 31 \% \\
\text { in PL; fewer } \\
\text { adverse events in } \\
\text { BMT group }\end{array}$ \\
\hline Gitlin (2001) & $\begin{array}{l}\text { Home education+ } \\
\text { environmental } \\
\text { modifications (IGr, } \\
93 ; \mathrm{TCC}, 78)\end{array}$ & $\begin{array}{l}\text { Typical } \\
\text { care }\end{array}$ & $\begin{array}{l}\text { RCT; } \\
3 \text { months }\end{array}$ & MBPC & $\begin{array}{l}\text { Reduction } \\
\text { behaviour in } \\
\text { intervention } \\
\text { group post-test }\end{array}$ \\
\hline Gendron (1996) & $\begin{array}{l}\text { Cognitive- } \\
\text { behavioural } \\
\text { intervention }(I G r \\
17 ; \mathrm{ISGr}, 18)\end{array}$ & $\begin{array}{l}\text { Support } \\
\text { group }\end{array}$ & $\begin{array}{l}\text { RCT; } \\
8 \text { weeks; } 3,6 \\
\text { months FU }\end{array}$ & MBPC & $\begin{array}{l}\text { No difference in } \\
\text { patient behaviour }\end{array}$ \\
\hline Teri (1997) & $\begin{array}{l}\text { Behavioural } \\
\text { treatment of } \\
\text { depression (BT-PE, } \\
\text { 23; BT-PS, 19; TCC, } \\
\text { 10; WLC, 20) }\end{array}$ & $\begin{array}{l}\text { Typical } \\
\text { care/ } \\
\text { Waiting- } \\
\text { list }\end{array}$ & $\begin{array}{l}\text { RCT; } \\
9 \text { weeks; } 6 \\
\text { months FU }\end{array}$ & $\begin{array}{l}\text { HDRS } \\
\text { CSDD } \\
\text { BDI }\end{array}$ & $\begin{array}{l}59 \% \text { improved in } \\
\text { BT-groups versus } \\
21 \% \text { in WLC, } \\
\text { maintained at } \\
\text { follow-up }\end{array}$ \\
\hline Huang (2003) & $\begin{array}{l}\text { PIST training } \\
\text { programme (IGr, } \\
24 ; C G r, 24)\end{array}$ & Yes & $\begin{array}{l}\mathrm{RCT} ; \\
2 \text { weeks, } 3 \\
\text { months FU }\end{array}$ & CMAl & $\begin{array}{l}\text { Sign. reduction in } \\
\text { agressive and } \\
\text { non-aggressive } \\
\text { behaviour and } \\
\text { agitation in } \\
\text { treatment group }\end{array}$ \\
\hline Bourgeois (2002) & $\begin{array}{l}\text { Skills training (PCh, } \\
\text { 22; SCh, 21; CGr, } \\
\text { 20) }\end{array}$ & Yes & $\begin{array}{l}\mathrm{RCT} ; \\
12 \text { weeks } 3,6 \\
\text { months FU }\end{array}$ & $\begin{array}{l}\text { Behave-AD } \\
\text { Caregiver } \\
\text { observation }\end{array}$ & $\begin{array}{l}\text { Reduced } \\
\text { frequency of } \\
\text { behaviour in PCh }\end{array}$ \\
\hline & & & & & $\begin{array}{l}\text { Fewer problems } \\
\text { in SCh than in } \\
\text { controls at post- } \\
\text { test and FU }\end{array}$ \\
\hline \multicolumn{6}{|l|}{ Psychoeducation } \\
\hline Gerdner (2002) & $\begin{array}{l}\text { Psychoeducation } \\
(\mathrm{IGr}, 132 ; \mathrm{CGr}, 105)\end{array}$ & Yes & $\begin{array}{l}\text { RCT; } \\
4 \text { weeks 3,6, } \\
9,12 \text { months } \\
\text { FU }\end{array}$ & MBPC & $\begin{array}{l}\text { Reduction in } \\
\text { frequency of } \\
\text { behaviour in } \\
\text { intervention } \\
\text { group }\end{array}$ \\
\hline Hebert (2003) & $\begin{array}{l}\text { Psychoeducation } \\
(\mathrm{IGr}, 79 ; \mathrm{CG}, 79)\end{array}$ & Yes & $\begin{array}{l}\mathrm{RCT} ; \\
15 \text { weeks }\end{array}$ & R-MBPC & $\begin{array}{l}\text { Reduction in } \\
\text { frequency of } \\
\text { behaviour in } \\
\text { intervention } \\
\text { group }\end{array}$ \\
\hline
\end{tabular}




\begin{tabular}{|c|c|c|c|c|c|}
\hline Study & $\begin{array}{l}\text { Intervention } \\
\text { (group size at } \\
\text { entry) }\end{array}$ & Control & $\begin{array}{l}\text { Design/ } \\
\text { duration }\end{array}$ & Scale & Outcome \\
\hline Ostwald (1999) & $\begin{array}{l}\text { Psychoeducation } \\
(\mathrm{IGr}, 50 ; \text { WLC, 30) }\end{array}$ & $\begin{array}{l}\text { Waiting- } \\
\text { list }\end{array}$ & $\begin{array}{l}\text { RCT; } \\
7 \text { weeks; 3,5 } \\
\text { months FU } \\
\text { post-baseline }\end{array}$ & R-MBPC & $\begin{array}{l}\text { No reduction in } \\
\text { frequency of } \\
\text { problem } \\
\text { behaviours }\end{array}$ \\
\hline \multicolumn{6}{|l|}{ Support groups } \\
\hline Hebert (1994) & $\begin{array}{l}\text { Support groups } \\
(\mid G r, 23 ; C G r, 18)\end{array}$ & Yes & 8 months FU & $\mathrm{R}-\mathrm{MBPC}$ & $\begin{array}{l}\text { No difference in } \\
\text { patient behaviour }\end{array}$ \\
\hline Quayhagen (2000) & $\begin{array}{l}\text { Cognitive } \\
\text { stimulation, dyadic } \\
\text { counseling, dual } \\
\text { support seminar, } \\
\text { early-stage day } \\
\text { care (CS, 21; DC, } \\
\text { 29; DSS, 22; EDC, } \\
\text { 16; WLC, 15) }\end{array}$ & $\begin{array}{l}\text { Waiting- } \\
\text { list }\end{array}$ & $\begin{array}{l}\text { RCT; } \\
8 \text { weeks } 3 \\
\text { months FU }\end{array}$ & MBPC & $\begin{array}{l}\text { No reduction in } \\
\text { patient behaviour }\end{array}$ \\
\hline
\end{tabular}

$\mathrm{ABID}=$ Agitated Behaviour Inventory for Dementia $\mid$ Behave-AD = Behavioural Abnormalities in Alzheimer's Disease rating scale $\mid \mathrm{BDI}=$ BeckDepression Inventory $\mid \mathrm{BMT}=$ Behavioural Management Training group $\mid$ BRSD = Behavioural Rating Scale for Dementia | BT-PE= Behaviour therapy-Pleasant events | BT-PS= Behaviour therapyproblrm solving | $\mathrm{CGr}=$ Control group $\mid \mathrm{CMAl}=$ Cohen-Mansfield Agitation Inventory $\mid \mathrm{CS}=$ Cognitive stimulation group $\mid$ CSDD $=$ Cornell Scale for Depression in Dementia $\mid D C=$ Dyadic Counseling group $\mid$ DSS = Dual Support group $\mid \mathrm{EDC}=$ Early-stage day care $\mid \mathrm{FU}=$ Follow up $\mid \mathrm{Hal}=$ Haloperidol $\mid$ HDRS = Hamilton Depression Rating Scale $\mid$ Igr = Intervention group | ISGr = Information Support group | (R-)MBPC= (Revised) Memory and Behaviour Problem Checklist $\mid \mathrm{PCh}=$ Patient Change group $\mid \mathrm{PL}=$ Placebo group $\mid \mathrm{PLST}=$ Progressively Lowered Stress Threshold Model $\mid \mathrm{RCT}=$ Randomized Controlled Trial $\mid$ Traz $=$ Trazodone $\mid \mathrm{TCC}=$ Typical Care Condition $\mid$ SCh =Self-Change group $\mid$ WLC $=$ Waitlist condition

\section{Intervention efficacy}

Results are only described of the thirteen studies with an adequate methodological quality. The differences in interventions and outcome measures do not allow for a statistical comparison between the study results.

The papers are arranged by type of intervention as presented in the article (some studies used more than one intervention group). The following interventions were identified: behavioural management training (8 studies; Bourgeois et al. 2002; Gendron et al. 1996; Gitlin et al. 2001; Gormley et al. 2001; Huang et al. 2003; Marriott et al. 2000; Teri et al. 2000; Teri et al. 1997), psychoeducation (3 studies; Gerdner et al. 2002; Hebert et al. 2003; Ostwald et al. 1999) support groups (2 studies; Hebert et al. 1994; Quayhagen et al. 2000), counselling (1 study; Quayhagen et al. 2000) and cognitive stimulation (1 study; Quayhagen et al. 2000). Most studies (62\%) reported a significant reduction in patient problem behaviours in the intervention group (see table 2). In addition, one of the nonsignificant studies did report a trend towards reduction in patient behaviour in the treatment group (Gormley et al. 2001). Results are presented in more detail for each intervention type. 
Behavioural management training

Eight articles reported on the effects of behavioural management training on patient problem behaviours. Methods ranged from introducing environmental modifications, teaching problem solving strategies, and increasing pleasant activities. All studies reported a beneficial effect of the intervention on patient behaviour. In the study of Gormley and colleagues (2001) only a tendency was found towards reduction in aggressive behaviour in the intervention group in comparison to the control group. In line with this, Huang and colleagues (2003) found only a decreasing trend in physically aggressive behaviours, but significantly lower levels of overall behavioural problems in the experimental group. Teri and colleagues (2000)compared the effect of behavioural management training with haloperidol, trazodone and placebo on patient agitation. They found no difference in improvement between the four groups, but fewer adverse effects were reported in the behavioural management group. Teri et al. (1997) examined in another study the effectivity of two non-pharmacological treatments of depression. It was found that the two active treatment conditions (behaviour therapy-Pleasant events and behaviour therapy-Problem solving) did not differ significantly from one another, but patient's depression significantly improved as compared with care-as-usual and the waiting list condition. Bourgeois and colleagues (2002) found intervention effects for aggressive/activity symptoms, but not for the psychosis/delusion items. Most studies found that significant reductions in the intervention group were maintained at 3 or 6 months follow up, except for the study of Marriot et al. (2000).

\section{Psychoeducation}

Three studies (Gerdner et al. 2002; Hebert et al. 2003; Ostwald et al. 1999) examined the effectiveness of a psycho-educational intervention. The psycho-educational intervention programmes consisted of a multicomponent approach including behavioural techniques. All studies used the (r-)MBPC as outcome measure to assess patient behaviour. Two studies (Gerdner et al. 2002; Hebert et al. 2003) found a significant reduction in frequency of problem behaviours in the intervention group. Gerdner and colleagues (2002) found that the intervention effect varied according to the relationship with the patient; a change in problem behaviours was reported by non-spousal caregivers only, with an increase in problem behaviours from baseline to 12 months follow-up in the control group and stable levels in the intervention group. In the study of Ostwald and colleagues (Ostwald et al. 1999) the intervention was successful in reducing negative caregiver reactions to problem behaviours, but was not successful in significantly reducing the behaviours itself. 
Support groups

Two studies (Hebert et al. 1994; Quayhagen et al. 2000) using a support group intervention found no significant differences in patient problem behaviours. In both studies the intervention consisted of 8 weekly sessions and the (r-)MBPC was used to assess patient behaviour. Hebert and colleagues (1994) compared the intervention group with a control group that attended informal monthly meetings of the Alzheimer's society. Quahagen and colleagues (2000) compared the support group condition with three other non-pharmacological interventions (cognitive stimulation, dyadic counselling and earlystage day care) and a wait-list condition. In this study only raw scores of patient problem behaviours were presented pre and post intervention, which showed no differences between the five groups.

\section{Discussion}

Twenty-six papers on caregiver interventions and patient problem behaviours fulfilled the inclusion criteria. Two articles were excluded because they duplicated two other studies. With respect to the remaining 24 studies there were several methodological limitations. First, group sizes were often too small to detect large effects. Only in five studies the study size was based on a power analysis. Second, many studies did not provide a detailed description of the target population, including information on caregiver kinship and contact intensity with the patient. Third, (information on) prognostic comparability between the intervention and control groups was often lacking. Fourth, in most studies the assessment of outcome measures was not blinded. Finally, few studies provided information on the comparability or avoidance of co-interventions such as the use of psychotropic medication in the patient. A rating of the methodological quality of the studies resulted in the inclusion of only 13 of 24 papers.

The majority of the 13 studies included in the review showed that caregiver interventions indeed can have beneficial effects on patient problem behaviours. Three types of caregiver training programs were evaluated: behavioural management training, psychoeducation, and social support groups. Behavioural training was examined most often and was found effective to reduce behavioural problems in the patient. Two out of three papers reporting on the effectiveness of a psycho-educational intervention also found a significant reduction in patient problem behaviour. No effect was found of support group programmes on patient behaviour.

The majority of the studies did not provide percentages of patients that improved after the intervention, thus unfortunately we were not able to calculate numbers-needed-to- 
treat (NNT) for these studies. Only Teri and colleagues $(1997,2000)$ provided proportions of improvement. They did not find a difference in patient agitation between a behavioural training group and placebo group (respectively $31.7 \%$ versus $30.6 \%$ improvement; NNT = 100). However, they did find a difference between two behavioural treatment conditions and a waitlist condition in patient depression (respectively 59\% versus $21 \%$ improvement; NNT $=2.5$.

There are several possible explanations for the positive effects of caregiver interventions on patient behaviour. Most psycho-educational programmes focus on understanding patient problem behaviours, which will change caregivers' interpretation of difficult behaviours and thus may decrease their irritation towards the patient and subsequently decrease patient inadequate behaviour. Behavioural training programmes not only increase caregiver understanding but also teach caregivers management skills and environmental modifications to reduce behavioural problems. In contrast, support groups are focusing more on supporting caregivers and alleviating their burden than on improving the caregiver-patient interaction, which probably makes this intervention type less successful in reducing patient problem behaviours. The relative effectiveness of behavioural management training is in line with the findings of Brodaty and colleagues (2003), who found in their meta-analysis on psychosocial interventions that intervention programmes focussing on the interaction between patient and caregiver are most successful in reducing caregiver psychological morbidity and delay patient institutionalisation. However, the findings concerning behavioural training must be interpreted cautiously, because three papers with the highest quality rating (Gormley et al. 2001; Marriott et al. 2000; Teri et al. 2000) showed only modest or no significant effects of the intervention. This is in line with findings of Acton et al. (Acton and Kang 2001) and Brown (1992), who found that more adequately designed studies have smaller effect sizes, because extraneous influences are controlled for; thus the intervention effect may be purer.

The majority of the studies focused on a reduction of total problem behaviours after intervention, whereas only a few studies examined the effect on specific symptoms. Effects of behavioural management training were found on agitation and depression, but not on psychosis or physical aggression. These differential effects suggest that subtypes of problem behaviours need to be taken into account when studying the effect of caregiver interventions on patient behaviour. Different types of interventions may be successful in reducing specific subtypes of behavioural symptoms. Striking was the lack of caregiver 
interventions focusing on apathetic symptoms in the patient, since apathy adversely affects the patient-caregiver relationship (Vugt et al. 2003) and is one of the most stressful symptoms to caregivers (Greene et al. 1982; LoGiudice et al. 1995). Adequate caregiver intervention studies are warranted that focus on patient apathy.

In some articles several interventions were compared within one study. Of special interest is the comparison of behavioural management training (BMT) with pharmacological treatment of agitation in the study of Teri et al. (2000). It was found that BMT was as effective as haloperidol, trazodone, or placebo, with fewer adverse effects in the BMT group. These results suggest that pharmacological treatments often wrongfully precede non-pharmacological interventions in clinical practice. Effectiveness of the synergy of combined pharmacological and non-pharmacological treatments has yet to be investigated.

In conclusion, this literature review shows that caregiver interventions, such as behavioural management training and psycho-education, are potentially successful in reducing patient problem behaviours. However, there is a lack of large, well-designed, controlled studies. In addition, there is a need for more refinement in the development of new caregiver interventions. Future studies should evaluate the effectiveness of interventions directed at more specific behavioural symptoms, such as patient apathy, as it is an important stressor for caregivers. Furthermore, interventions should be adjusted to caregiver characteristics as well, because caregivers differ in their care strategies (Vugt et al. 2004) and subsequently have different needs. Tailoring interventions for patient as well as caregiver characteristics will probably increase their effectiveness.

\section{Key points}

- The majority of caregiver intervention studies lack methodological rigour

- Caregiver interventions, especially behavioural management training, show beneficial effects for patient behavioural problems 
Chapter 11. Impact of caregivers on problems in dementia: a qualitative review 


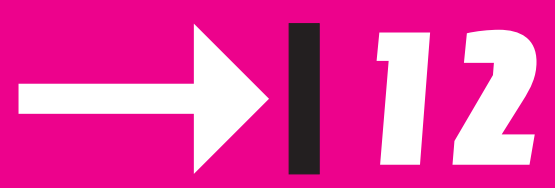

General discussion 


\section{Introduction}

Two-third of the dementia patients are cared for at home by family members. Caring for a demented person who has problematic behaviour poses great demands and has detrimental consequences for many caregivers. Caregivers differ in their competence and skills to successfully manage problem behaviours and may even trigger or exacerbate difficult behaviours in the patient. This thesis focuses on the interaction between caregiver functioning and patient behavioural problems. This is a two way interaction, of which both sides will be dealt with separately. First, we examined the impact of patient behavioural problems on caregiver distress, the relationship quality, and the decision to institutionalize the patient (chapters 4, 5, 6, and 7). Next, we focused on the consequences of several aspects of caregiver functioning on the course of the behavioural symptoms in the patient (chapters 8, 9 and 10). We further examined the effect of modifying caregiver impact on patient behaviour, by reviewing the literature (chapter 11) on the effectiveness of caregiver interventions to reduce patient problem behaviours. In this last chapter of the thesis we will briefly summarize the findings and discuss methodological limitations, implications for clinical practice and future directions.

\section{Summary of findings}

\section{The impact of patient behaviour on caregiver functioning}

With regard to the negative impact of patient behavioural problems on various aspects of caregiver functioning, as addressed in chapter 4, 5, 6, and 7, results show that patient behavioural problems are distressing to caregivers, as indicated by subjective as well as objective measures.

The finding in chapter 4 that high levels of patient behavioural problems are associated with a higher caregiver cortisol awakening response provides evidence for a physiological stress response in caregivers, in addition to subjective stress. The observed elevation of cortisol levels is an important finding, because it might increase caregivers' susceptibility to physical health problems, as chronic exposure to stress hormones increases disease vulnerability (Bauer et al. 2000).

A further examination of the impact of specific behavioural symptoms in chapter 5 and 6 indicated that specific symptoms have specific effects on family caregivers, with patient apathy being one of the most important symptoms in this regard. Caregivers of Alzheimer's Disease (AD) patients as well as caregivers of Frontotemporal dementia (FTD) 
patients reported that patient symptoms of depression and apathy were most distressing to them (chapter 6). Furthermore, patient apathy was the symptom that adversely affected the quality of the spousal relationship the most, by causing a loss of essential relationship elements, such as joint activities, shared experiences, and verbal communication. The negative impact of patient apathy on caregiver functioning is an important finding, as clinical practice and intervention research have mainly focused on the impact of excessive patient behaviours, such as aggression and restlessness and have under recognized the importance of apathy so far. Only few other studies reported that apathetic and withdrawn behaviour was most stressful to caregivers (Greene et al. 1982; Landes et al. 2001; LoGiudice et al. 1995). However, most caregiver studies did not take these patient behaviours into account. One might hypothesize that the importance of patient apathy to caregiver burden is caused by the fact that it is too often unrecognized as a symptom of the underlying disease process, as it has received little attention in clinical practice and intervention research as such. Thus, patient apathy may be often misinterpreted as intentional, lack of commitment or laziness and thus creating more caregiver stress (Landes et al. 2001). In addition, apathetic patients place heavy demands on their caregivers, because they depend on others to initiate activities even when they are still capable to perform the activities themselves (Landes et al. 2001).Furthermore, caregivers may find it difficult that their relationship with the patient becomes unidirectional and that they do not get any feedback from the patient on their care activities.

High levels of caregiver distress appeared to have profound consequences for patient and caregiver (chapter 7), as caregiver distress related to patient behaviour was a significant predictor of nursing home placement, while patient behaviour in itself was not. These results underscore the importance of distinguishing objective burden (behavioural problems) from the resulting subjective distress in the caregiver, when estimating the risk for institutionalization. In addition, kinship has to be taken into account in this regard, because daughters institutionalized the patient sooner than spousal caregivers. This finding indicates that taking care for a dementia patient is more difficult to bear for daughters than for spousal caregivers, which may be caused by a lower commitment to care or more competing life responsibilities in daughter caregivers compared to spouses (Pot 1996). 


\section{The impact of caregiver factors on patient behaviour}

Besides the finding that patient behaviour has profound consequences for family caregivers, caregivers themselves are also, vice verse, likely to play a role in the emergence of patient behavioural problems. With regard to caregiver management strategies (chapter 8), it was found that non-adapting caregivers were most likely to encounter patient hyperactivity symptoms during the course of the dementia and felt least competent compared to nurturers and supporters, whereas supporters reported the lowest levels of patient hyperactivity and felt most competent as a caregiver. These findings resulted in the identification of the most vulnerable patient-caregiver group, characterized by a non-adapting caregiver strategy, feelings of low caregiver competence, and high levels of patient hyperactivity. It is most likely that there exists a mutual negative interaction in these vulnerable couples, with both patient and caregiver reacting with irritation and anger towards each other, resulting in an exacerbation of patient hyperactivity. This is further supported by the finding in chapter 9 that critical/overinvolved caregivers, as indicated by a measure of expressed emotion (EE), also reported higher levels of patient hyperactivity and lower feelings of competence. Although not mentioned in chapter 8 or 9 , we hypothesize at this point that non-adapting caregivers were probably most likely to have high levels of EE. Therefore, a post-hoc analysis was performed, which indeed showed that there was a significant difference between the management style groups in levels of $\mathrm{EE}(\mathrm{X} 2=8.5, \mathrm{df}=2, \mathrm{p}=0.014)$, with $50 \%$ of the nonadapters, $43 \%$ of the nurturers, and only $18 \%$ of the supporters scoring high on $\mathrm{EE}$.

In addition, results in chapter 10 showed that vulnerability was also increased by suboptimal cognitive functioning in spousal caregivers. Suboptimal memory performance in caregivers was related to an increase in patient hyperactivity symptoms. This finding is in line with the results described in chapter 8 and 9, that caregiver functioning is associated with patient hyperactivity. The fact that suboptimal cognitive functioning preceded an increase in patient hyperactivity supports the notion that caregiver functioning plays a role in the etiology of patient behaviour. But again, considering the fact that there is a reciprocal interaction between patient and caregiver it seems most plausible that there exists a mutual relationship between patient problem behaviours and caregiver functioning.

The influence of the care environment on patient behaviour can be seen from the theoretical perspective provided by the ecological model of Lawton (Lawton 1975). According to this model, behaviour is a function of the competence to deal with the demands from the environment. Dementia patients are particularly sensitive to the 
impact of the environment, because of their diminished competence and increased dependence. Consequently, a low competence or high demand from the environment may result in inadequate behaviours. The results from the current study may then imply that caregivers with a non-adaptive strategy, high levels of expressed emotion, or suboptimal cognitive functioning may not be able to counterbalance the lowered competence in the dementia patient, whereas supporting caregivers are most competent to adapt to the needs of the patient and create a safe environment.

Further evidence for the impact of caregiver functioning on patient behaviour is provided by caregiver intervention studies. A review of caregiver intervention studies in chapter 11, including behavioural management training, psycho-education, and social support groups, suggested that patient problem behaviours can be improved by teaching caregivers adequate management skills. This underlines the importance of the MAASBED results, as the identification of vulnerable patient-caregiver couples can be used as a starting point for caregiver interventions to improve caregiver skills and subsequently reduce patient problem behaviour.

\section{Methodological considerations}

MAASBED has several methodological limitations that need to be addressed. Methodological problems and issues will be discussed, as well as how they may have influenced the results presented in the various chapters of this thesis.

\section{Sample selection}

We subsequently included subjects in MAASBED from the Memory Clinic of the Academic Hospital Maastricht, as well as from the geriatric division of the Regional Institute for Community Mental Health (RIAGG), to enclose as much dementia patients as possible living in the Maastricht region. However, it is likely that a selection was made, because patients diagnosed with dementia by the general practitioner but not referred to the hospital or the RIAGG were not included in the study. General practitioners are likely to make a selection when referring a patient to an institution, with more severely demented patients, patients with behavioural problems, or caregivers in need for support being more easily referred. Considering the fact that this thesis deals with patient behavioural symptoms and negative interpersonal interaction, perhaps our results are not valid for all community-living dementia patients and their caregivers. Although we may have over sampled patients with problem behaviours or caregivers with management problems, the 
results, however, are still representative for the included settings and useful for clinical practice. Moreover, we were particularly interested in this problematic group.

Sampling bias may have also occurred by selective participation of caregivers. Since excessive time demands are one of the most prominent problems in caregiving (Dura and Kiecolt-Glaser 1990), participating in time demanding research may be problematic for many caregivers. Similarly, providing care for a dementia patient often results in a decreased mobility. Data from a study of Dura and colleagues (1990) showed that caregiver studies that limit participation to those who are willing to travel to a central assessment site may bias their sample towards less depressed caregivers who provide care for patients with fewer dementia-related problems. To maximize participation, all subjects in MAASBED have been offered at-home assessments. Still, $24 \%$ of the caregivers in the total patient sample refused to participate. As the most important reason for refusal was emotional burden, it is likely that non-participants represent a more distressed subpopulation of caregivers.

Furthermore, the included sample was heterogeneous with respect to caregiver gender, kinship, contact intensity with the patient, and dementia diagnosis. The heterogeneity of the sample may have obscured associations between caregiver functioning and patient behaviour. For example, one might expect to have found a stronger association between caregiver functioning and patient behaviour when only caregivers with high patient contact intensity were included. However, this would have reduced the sample size and made the sample less representative for the whole caregiver population. Therefore, it was decided to take these variables into account in the statistical analysis as possible confounding variables. In addition, sub-samples where used when appropriate.

\section{Assessment of behavioural symptoms}

The assessment of behavioural symptoms in dementia presents some difficulties. Dementia patients themselves are not a reliable source of information, because they may not exhibit, remember or report their symptoms at the time of the interview. Therefore, proxy interviews with a caregiver or other informant are primarily used to assess changes in patient behaviour. Caregiver reports of some neuropsychiatric symptoms, such as delusions or hallucinations, must then be inferred from behaviour if not described by the patient (Cummings 1994). Furthermore, caregiver reports may be affected by feelings of burden, guilt, or denial. Despite this possibility of confounding, caregivers as a group have 
been reported as a reliable source of information (Zarit 1996). But, alternatively, more objective measures of patient behaviour are needed to assess the reporting bias in caregivers. On the other hand, the subjective experiences of the caregiver are even more important than objective assessment to identify vulnerable patient-caregiver couples in clinical practice.

In addition, the reliability of the reports is greatly depending on the instrument used. In MAASBED the Neuropsychiatrc Inventory (NPI) was used to measure the neuropsychiatric symptoms in dementia. This is one of the most widely used instruments to assess behavioural changes. The NPI has the advantage of asking about a wide range of behaviours that are specific and observable. The instrument consists of detailed questions about the specific aspects of each behavioural domain and differentiates between severity, frequency and emotional burden of the caregiver of behavioural changes. The combination of symptom frequency and severity is reported to be a stronger predictor of caregiver distress than symptom frequency alone (Cummings 1994).

One of the goals of this thesis was to differentiate between specific behavioural problems instead of using a total score of symptoms. Therefore a factor analysis was performed to detect behavioural sub syndromes of the NPI (chapter 3). The identification of three sub syndromes provided a data-reduction of the $12 \mathrm{NPI}$ symptoms in subsequent analysis, but still enabled us to examine the differential impact of specific behaviours. However, caution is needed in studying patient behaviour at a syndromal level, because the robustness of the syndromes across studies and across dementia stages has yet to be demonstrated. Furthermore, the use of individual symptoms may be preferable when high specificity is useful. For example, in chapter 5 we found an effect of the mood/apathy sub syndrome on negative changes in the spousal relationship. When further examining the underlying mechanisms of this association it appeared that negative relationship changes were associated with patient apathy rather than mood problems. This finding indicates that although apathy and mood problems tend to occur together in the same patient and may arise from a common underlying construct, they have a differential impact on the environment and therefore they need to be discriminated in this regard.

The fact that we failed to find any association between caregiver factors and patient psychosis needs some further reflection. One might hypothesize that psychotic symptoms may be experienced by caregivers as less distressing than mood/apathy symptoms, because they may be more obvious symptoms of the underlying illness and therefore interpreted as unintentional. With regard to the underlying pathogenesis of psychotic symptoms, they may be seen as the result of biological factors rather than 
triggered by a poor interpersonal interaction. However, one cannot preclude that the lack of association with psychosis is due to a type two error, related to the low frequency of these symptoms in the sample. Over the course of the follow-up period psychotic symptoms were present in only around $50 \%$ of the patients. It is possible that with a larger sample and therefore an increased statistical power, we would have found significant associations between caregiver functioning and patient psychosis.

\section{Follow-up}

One of the problems with longitudinal designs is the loss to follow-up. When there is a large proportion of the sample dropping out or when the dropout is selective, the results of the study are likely to be biased. In our sample the proportion of caregivers lost at 1 year follow-up was 19\% and at 2 years follow-up 37\% (see chapter 2). Several patient and caregiver characteristics were compared between the dropouts and 'stayers' to identify selective attrition. It was found that caregivers lost to follow up were older and patients were more severely demented. Although no differences were found at baseline in our main outcome measure (NPI) this dropout has probably biased the results by selecting the more mildly impaired patients and younger caregivers. For example, in chapter 10 the selection of younger caregivers may have resulted in an underestimation of the cognitive problems in spousal caregivers and the impact on patient problem behaviours.

\section{Causality}

In this thesis, behavioural problems in dementia were examined in a social care context. Cross-sectional data were used to assess the impact of patient behaviour on caregiver burden. A longitudinal design was used to study patient behaviour as a predictor of institutionalization during the two year follow-up. Furthermore, caregiver factors were examined longitudinally as predictors of patient behaviour. Examination of the temporal relationship between caregiver factors as predictors of patient behaviour is necessary to determine causality. Still, causality could not be fully determined since differences in patient behaviour often already existed at baseline (see chapter 8 and 9). One could argue that these baseline differences should be adjusted for in the analysis to determine causality. However, baseline measurements were not equal to the starting point of the interactive process between patient and caregiver, as mean dementia duration was 36 months at baseline assessment. Therefore, correcting for baseline differences would presumably reduce the sensitivity to detect significant associations between patient behaviour and caregiver functioning. Moreover, determining the exact direction of 
causality may be less relevant in daily practice, as the results of MAASBED has led to the identification of vulnerable client systems, in which probably a circular pattern of causation exists.

\section{Combining qualitative and quantitative methods}

In chapter 5 and 8 of this thesis qualitative and quantitative research were used in a complementary way. The combined use of these methods is addressed here, because both approaches are sometimes considered to be antithetical instead of complementary. Qualitative methods score high on validity, emphasize the meanings and views of all research participants (Pope and Mays 1995), and are therefore particularly suitable to learn more about the experiences of caregivers of dementia patients. Quantitative methods are more standardized and score higher on reliability and generalizability. Qualitative and quantitative methods can be combined in several ways. In chapter 5, qualitative analysis followed quantitative results to further explore the underlying mechanism of the association between patient mood/apathy symptoms and negative relationship changes. On the other hand, quantitative methods can build on qualitative results, as the qualitative identification of caregiver strategies in chapter 8 was followed by a quantitative examination of the strategies. Combining both methods allows access to different levels of knowledge and helps to create a wider picture (Pope and Mays 1995). Thus, the differences in methodology of both approaches do not imply that quantitative and qualitative approaches are incompatible, but can actually be used to strengthen each other, as we have proved here.

\section{Medication use}

In view of the naturalistic nature of the current study, no treatments affecting patient behavioural symptoms were excluded. For example, patients used a various range of medications such as antipsychotics, antidepressants and benzodiazepines. While a confounding influence of medication use cannot be precluded, it probably resulted in an underestimation of the negative impact of caregiver factors on patient behaviour. Namely, successful treatment will decrease the severity of the behavioural problems and therefore reduce the negative impact of caregiver factors.

\section{Conceptual considerations}

Several conceptual issues will be discussed here. First, as behavioural problems in dementia are highly prevalent, and have important consequences for patient and 
caregiver, they need to be considered as an intrinsic part of dementia instead of secondary symptoms or complications of dementia. A behavioural approach to dementia will yield more treatment options for patient and caregiver than a mainly cognitive perspective.

Second, the results described in this thesis stress the importance of differentiating between specific behavioural syndromes or symptoms in dementia. However, with the introduction of the term BPSD by the IPA in 1996, behavioural problems in dementia were implicitly considered to be a unitary entity. Although the term BPSD has served a useful purpose by increasing the awareness of the importance of behavioural symptoms in dementia, at this point the focus of attention has to expand beyond BPSD to specific symptoms or syndromes, with each its typical biological correlates, and psychological and social consequences.

Furthermore, regarding the etiology of behavioural symptoms, the most plausible model is multidimensional and includes biological, psychological, and environmental aspects (Lawlor 1996). Although the contributing role of each factor still needs further investigation, emphasis on an interactive model of patient problem behaviours is important in view of the development of intervention programs. From this standpoint, we advocate a change in perspective from seeing dementia as a primarily neurological disorder with behavioural consequences, to emphasizing the complex interpersonal dynamics between patient and caregiver, in which a circular pattern of causation exist. A too narrow focus is unwarranted and unethical, as it leaves important treatment options unused.

\section{Clinical implications}

The results described in this thesis have several implications for clinical practice. Research findings concerning the prevalence of patient behavioural problems and their impact on caregivers, stress the importance of monitoring behavioural symptoms in dementia in clinical practice routine. Incorporation of behavioural measures into dementia test protocols is therefore warranted. Standardized assessment of behavioural problems and related caregiver distress over time should serve as an important indicator to clinicians for the need for possible interventions.

Differential effects of specific patient behaviours suggest that more emphasis must be placed on individual symptoms or syndromes rather than on behavioural problems as a unitary entity ('BPSD'). One of the most important individual symptoms is apathy, as it frequently occurs in dementia, induces caregiver distress, and adversely affects the 
patient-caregiver relationship. Unfortunately, apathy in dementia has received too little attention in caregiver research and clinical practice. Most intervention studies and recommendation reports focus on excessive patient behaviours such as aggression and restlessness (Brodaty 2003), while apathetic patients are often considered to be 'easy' patients, as is probably true for a professional care situation. But also in a professional care situation apathy may have a negative impact as it may decrease patient quality of life. The current results imply that clinicians need to be more aware of the negative impact of patient apathy. Furthermore, there is a need for development of intervention programs that target apathy in dementia patients, for instance early activation of patients, and caregiver psycho-education may be effective in this respect.

In view of the results on patient institutionalization, it is assumed that interventions aimed at reducing caregiver distress related to problem behaviours may have a beneficial effect on the institutionalization rate of dementia patients. In addition, the findings stress the importance of differentiating between spousal and non-spousal caregivers. Thus, interventions should be tailored to the type of caregiver and the specific problems they experience.

Another important clinical implication of the findings in this thesis is the identification of vulnerable patient-caregiver couples. These couples can be characterized by several caregiver and patient characteristics, such as suboptimal caregiver cognitive functioning, poor interpersonal interactions between patient and caregiver, low feelings of competence in caregivers, and exacerbation of patient hyperactivity. There probably exists a continues interactive relationship between suboptimal caregiver functioning on the one hand, and negative patient behaviour on the other. The role of interactive aspects in the etiology of behavioural problems in dementia, have been a neglected area and warrant more emphasis in clinical practice. The identification of vulnerable patientcaregiver systems and the differentiation in caregiver management strategies may provide useful starting points for the development of more specific caregiver interventions. Psycho-education and skills training is most needed in non-adaptive, high EE caregivers to increase feelings of competence and reduce patient hyperactivity. But also nurturing caregivers might benefit from learning more "supporting" skills, as supporting caregivers were found to be most successful. Furthermore, one should be alert to cognitive problems in older caregivers as they may be less able to provide adequate care. 


\section{Future directions}

The Behavioural and Psychological Symptoms in Dementia (BPSD) include a heterogeneous range of behaviours and psychological reactions (Finkel et al. 1996). In view of the differential etiology and impact of specific behaviours, future research must continue to look beyond BPSD and focus on specific symptoms or syndromes, rather than on an unitary syndrome of BPSD. Apathy is an important symptom in this regard, because it was found to have profound consequences for caregiver functioning. Furthermore, there is a striking lack of psychosocial interventions in this regard. Therefore, specific intervention programs will be needed to target apathy and reduce caregiver distress.

As the caregiver role can be described as a career process with different stages and changing perceptions (Lindgren 1993), the impact of behavioural symptoms should also be studied longitudinally. Furthermore, research into the impact of behavioural problems on the environment needs to expand to professional care situations. It is expected that professional caregivers differ in their perception of behavioural problems from family caregivers and probably find patient apathy less distressing.

More research into what constitutes a successful intervention is needed. Successfulness might be increased by using a system-approach and targeting vulnerable patientcaregiver couples. The findings of our study will help to identify these vulnerable systems and may be useful to develop more specific interventions for different patient-caregiver types.

Furthermore, evidence-based clinical guidelines for the management of behavioural problems in dementia are needed. In current clinical practice there is often a lag between evidence and practice. Moreover, many clinical guidelines are based on individual clinical expertise rather than on external evidence. The findings from MAASBED have clear practical implications, as discussed in this last chapter, that might contribute to the development of more evidence-based guidelines.

\section{Conclusions}

The behavioural and psychological symptoms in dementia have negative consequences for caregiver functioning. Specific behavioural symptoms or syndromes differ in their impact on caregivers, with apathy being one of the most important symptoms in the mild to moderate stages of dementia. Patient apathy induces caregiver distress and has an adverse effect on the patient-caregiver relationship. Furthermore, caregiver distress related to patient problem behaviours is an important predictor of patient 
institutionalization. Striking is the difference between daughter caregivers and spouses, with daughters institutionalizing the patient sooner.

The importance of an interactive model of patient problem behaviours is stressed throughout various chapters of this thesis. Vulnerable patient-caregiver couples can be characterized by suboptimal caregiver cognitive functioning, diminished feelings of caregiver competence, poor interpersonal interaction, and exacerbation of patient hyperactivity symptoms. The differentiation in nurturing, supporting, and non-adapting management strategies may be useful in the development of more specific caregiver interventions. Caregiver intervention studies show the potential to reduce patient behavioural problems, but so far lack methodological rigor.

Future studies should continue to concentrate on differential aspects of specific behavioural symptoms or syndromes. Patient apathy is an important but neglected symptom in clinical practice and intervention studies, and therefore should be an important focus in both areas. A change in perspective from a medical-biological viewpoint to an interactive model of patient behaviour in dementia may provide more opportunities for the development of successful interventions to improve the quality of life of both patient and caregiver. Furthermore, methodological improvement and refinement in intervention studies is necessary. Tailored intervention programs are needed that target vulnerable patient-caregiver systems. 
12 
Summary 
Summary

\section{Introduction}

This thesis deals with the behavioural and psychological changes in dementia in relation to caregiver functioning. Behavioural and psychological problems occur frequently during all stages of the dementia process, and include a heterogeneous range of behaviours, such as depression, apathy, agitation, aggression, psychosis, hallucinations, and restlessness. The majority of the dementia patients are cared for at home by a family member, mostly spouses or children. Caring for a demented person with problem behaviours is very demanding for family caregivers and has profound consequences. Furthermore, caregivers differ in their competence to adequately manage patient problems, and may even worsen problem behaviours. The topic of this thesis is the twoway interaction between patient behaviour and caregiver functioning, of which both sides are dealt with separately. Thus the two main questions addressed in this thesis are:

1. What is the impact of behavioural problems in dementia patients on the family caregiver?

2. What is the impact of family caregivers on patient problem behaviours in dementia?

To examine the interaction between patient behaviour and caregiver functioning, we performed a longitudinal study into the course and risk factors of behavioural problems in dementia, entitled the Maastricht Study of Behaviour in Dementia (MAASBED). A description of MAASBED is provided in chapter 2 of this thesis.

In chapter 3 we tried to identify distinct behavioural syndromes, using the NeuroPsychiatric Inventory (NPI). The three subsyndromes that were found (hyperactivity, mood/apathy, and psychosis) formed the basis of further analyses described in this thesis.

\section{The impact of patient behaviour on caregiver functioning}

The first aim of this study was to assess the negative impact of patient behavioural problems on various aspects of caregiver functioning, as addressed in chapter 4, 5, 6, and 7. Results show that patient behavioural problems are the most distressing to caregivers, as indicated by subjective as well as objective measures. Furthermore, caregiver distress appeared to have profound consequences, as it increased the risk of patient institutionalization.

In addition, it was found in chapter 4 that high levels of patient behavioural problems led to a higher caregiver cortisol awakening response, an objective indicator of stress (Pruessner et al. 1997), mediated through stress appraisal. The observed elevation of 
cortisol levels is an important finding, because it could increase caregivers' susceptibility to physical health problems, as chronic exposure to stress hormones increases disease vulnerability (Bauer et al. 2000). These findings provide evidence for a physiological stress response in caregivers, in addition to subjective measures.

A further examination described in chapter 5 and 6 indicated that specific symptoms have specific effects on family caregivers, with patient apathy being one of the most important symptoms in this regard. Caregivers of Alzheimer's Disease (AD) patients as well as caregivers of Frontotemporal Dementia (FTD) patients reported that patient symptoms of depression and apathy were most distressing to them (chapter 6). In addition, disinhibition was severely distressing in FTD caregivers, whereas anxiety was typically distressing in AD caregivers. FTD caregivers were overall more distressed by the behaviour of their partner than AD caregivers. Thus, individual behavioural symptoms in dementia patients have a differential impact on their caregivers, with mood disturbance and apathy having overall the greatest impact. But, besides these similarities also differences exist between specific diagnostic groups, with behaviour related distress being most prominent in FTD caregivers.

Furthermore, patient apathy was the most important symptom that adversely affected the quality of the spousal relationship, by causing a loss of essential relationship elements, such as joint activities, shared experiences, and verbal communication. Results indicated that caregivers better cope with excessive patient behaviour that disrupts interaction, than they are with a decrease in interaction because of diminished conversation or disinterest on the part of the patient.

High levels of caregiver distress appeared to have profound consequences for patient and caregiver. The study described in chapter 7 showed that caregiver distress related to patient behaviour was a significant predictor of nursing home placement, while patient behaviour in itself was not. These results stress the importance of distinguishing objective burden (in terms of behavioural problems) from the resulting subjective distress in the caregiver, when estimating the risk for institutionalization. In addition, kinship has to be taken into account in this regard, because daughters institutionalized the patient sooner than spousal caregivers.

\section{The impact of caregiver factors on patient behaviour}

Besides the finding that patient behaviour has profound consequences for family caregivers, they are, vice verse, likely to play a role in the emergence of patient behavioural problems. The impact of several aspects of caregiver functioning on patient 
Summary

behaviour was addressed in the second part of this thesis (chapter 8,9,10, and 11). Results from these studies suggest that caregiver competencies and abilities to provide adequate care may affect hyperactive behaviour in the patient. No evidence was found that patient mood/apathy or psychosis was influenced by caregiver functioning.

First, differences in caregiver management styles were examined in chapter 8, which resulted in the identification of the following three strategies, on the basis of analysis of verbatim reports of in-depth interviews in caregivers: (1) caregivers using a 'nurturing' strategy felt that they were taking care of a child, no longer regarded the patient as equal, and focused on personal care tasks or protection of the patient; (2) a 'supporting' strategy was characterized by adaptation to the patient's level of functioning, supervising the patient, and stimulating his or her abilities; (3) and a 'non-adapting' strategy was characterized by a lack of understanding of the problems, and irritation or impatience towards the patient. We found that caregiver characteristics such as sex, education, and personality were important determinants of management strategies. Furthermore, possible consequences of these management styles were examined. We found that nonadapting caregivers were most likely to encounter patient hyperactivity symptoms during the course of the dementia and felt least competent compared to nurturers and supporters, whereas supporters reported the lowest levels of patient hyperactivity and felt most competent as a caregiver. These findings resulted in the identification of the most vulnerable patient-caregiver group, characterized by a non-adapting caregiver strategy, feelings of low caregiver competence, and high levels of patient hyperactivity. It is most likely that there exists a mutual negative interaction in these vulnerable couples, with both patient and caregiver reacting with irritation and anger towards each other, resulting in an exacerbation of patient hyperactivity. This is further supported by the finding in chapter 9 that highly critical/over-involved caregivers, as indicated by a measure of expressed emotion (EE), also reported higher levels of patient hyperactivity and lower feelings of competence.

In addition, vulnerability may be increased by suboptimal cognitive functioning in caregivers. The continuously changing and demanding care situation requires optimal cognitive functioning in caregivers. Spousal caregivers of dementia patients are usual of the same age as the patient and therefore at risk for age-related cognitive decline (Jolles 1986), which may affect their ability to provide adequate care. Examination of caregiver cognitive functioning and possible consequences for patient behaviour in chapter 10 showed that suboptimal memory performance in caregivers was related to an increase in patient hyperactivity symptoms. This finding is in line with the results described in chapter 
8 and 9, that caregiver functioning is associated with patient hyperactivity. The fact that suboptimal cognitive functioning predicted an increase in patient hyperactivity supports the notion that caregiver functioning plays a role in the etiology of patient behaviour. But again, considering the fact that there is a reciprocal interaction between patient and caregiver it seems most plausible that there exists a mutual relationship between patient problem behaviours and caregiver functioning.

The results from the current study imply that caregivers with a non-adaptive strategy, high levels of expressed emotion, or sub-optimal cognitive functioning may not be able to counterbalance the lowered competence in the dementia patient, whereas supporting caregivers are most competent to adapt to the needs of the patient and create a safe environment.

Further evidence for the impact of caregiver functioning on patient behaviour is provided by caregiver intervention studies. A review of caregiver intervention studies in chapter 11, including behavioural management training, psycho-education, and social support groups, suggested that patient problem behaviours can be improved by teaching caregivers adequate management skills. This underlines the importance of the MAASBED results, as the identification of vulnerable patient-caregiver couples can be used as a starting point for caregiver interventions to improve caregiver skills and subsequently reduce patient problem behaviour.

\section{Discussion and conclusion}

Methodology, findings and their relevance are discussed in chapter 12. Methodological issues included the sample selection, assessment of behavioural symptoms, loss to followup, causality, combination of qualitative and quantitative methods, and the confounding influence of medication use.

Patient problem behaviours have negative consequences for caregiver functioning, with patient apathy being the most important symptom in this regard. Several explanations for the contribution of apathy to caregiver burden are discussed. The impact of apathy is an important finding, as clinical practice and intervention research have under-recognized the importance of apathy so far. Furthermore, the differential effects of specific patient behaviours stress the importance of focusing beyond BPSD on individual symptoms or syndromes.

As caregiver functioning is, vice verse, likely to play a role in the emergence of patient behavioural problems, the importance of an interactive model of patient problem behaviours was set forth. Vulnerable patient-caregiver systems were found, characterized 
Summary

by suboptimal caregiver functioning, diminished feelings of caregiver competence, poor interpersonal interaction, and higher levels of patient hyperactivity. The identification of vulnerable client systems may provide a useful starting point for the development of more specific interventions.

Overall, these findings illustrate that behavioural changes are a prominent aspect of dementia, and therefore should be considered as an intrinsic part of the disease. A behavioural approach to dementia, with emphasis on an interactive model of patient behaviour, will provide most opportunities for successful interventions to increase the quality of life of both patient and caregiver. 
Samenvatting 


\section{Introductie}

Het onderzoek dat in dit proefschrift wordt beschreven betreft de relatie tussen enerzijds gedragsmatige en psychologische veranderingen bij dementie en anderzijds het functioneren van de mantelzorger. Gedragsmatige en psychologische problemen komen frequent voor gedurende alle stadia van het dementieproces. Het betreft een breed scala aan verschijnselen, zoals depressie, apathie, agitatie, psychose, hallucinaties en rusteloosheid. Deze gedragsproblemen vormen vaak een belangrijke bron van stress voor de omgeving. De meerderheid van de dementie patiënten wordt thuis verzorgd door een familielid, meestal de partner of een van de kinderen. Zorgen voor een dementerende met gedragsproblemen eist veel van familie en heeft ingrijpende consequenties. Bovendien is het zeer waarschijnlijk dat mantelzorgers verschillen in hun competenties om adequate zorg te bieden en problemen op te lossen, en kunnen zij mogelijk zelfs probleemgedrag in de patiënt verergeren. Het onderwerp van dit proefschrift is enerzijds de impact van gedragsproblemen bij de patiënt op het functioneren van de mantelzorger en anderzijds de invloed van de mantelzorger op probleemgedrag van de patiënt. Beide richtingen van de interactie zullen aan de hand van verschillende aspecten apart aan de orde komen. Dus de twee hoofdvraagstellingen van dit proefschrift zijn:

1. Wat is de impact van gedragsproblemen bij dementie op het functioneren van de mantelzorger?

2. Wat is de invloed van mantelzorgers op het ontstaan en beloop van gedragsproblemen bij dementie?

Deze vraagstellingen zijn onderzocht in een longitudinaal onderzoek naar het beloop en de risicofactoren van gedragsproblemen bij dementie, genaamd de Maastricht Study of Behaviour in Dementia (MAASBED). Een beschrijving van MAASBED wordt gegeven in hoofdstuk 2 van dit proefschrift.

In hoofdstuk 3 is getracht om verschillende gedragssyndromen te identificeren op basis van de meting van 12 gedragssymptomen met behulp van de NeuroPsychiatric Inventory. De drie subsyndromen die werden gevonden (hyperactiviteit, stemming/apathie, en psychose) vormen de basis voor de verdere analyses die beschreven staan in dit proefschrift. 


\section{De invloed van probleemgedrag bij dementie op de mantelzorger}

De eerste doelstelling van de studie was het onderzoeken van de invloed van probleemgedrag van de dementerende op het functioneren van de mantelzorger, zoals beschreven in hoofdstuk 4, 5, 6, en 7. De resultaten van zowel objectieve als subjectieve metingen laten zien dat gedragsproblemen bij dementerenden stresserend zijn voor mantelzorgers. Een hogere mate van emotionele belasting bij de mantelzorger blijkt vervolgens een risicofactor te zijn voor eerdere opname van de patiënt.

De resultaten in hoofdstuk 4 geven aan dat een hogere mate van gedragsproblemen bij de patiënt leidt tot een hogere cortisol respons na ontwaken bij de mantelzorger, hetgeen een objectieve indicator is voor stress (Pruessner et al. 1997), gemedieerd door de subjectieve ervaring van stress. De geobserveerde verhoging van cortisol is een belangrijke bevinding, aangezien chronische blootstelling aan stresshormonen een verhoogde kwetsbaarheid geeft voor ziektes (Bauer et al. 2000) en dus de mantelzorger gevoelig maakt voor lichamelijke problemen.

De resultaten beschreven in hoofdstuk 5 en 6 geven aan dat specifieke symptomen ook specifieke effecten hebben op mantelzorgers, waarbij apathie een van de symptomen is met de grootste impact op de mantelzorger. Zowel mantelzorgers van patiënten met de ziekte van Alzheimer (AD) als mantelzorgers van Frontaalkwab dementie patiënten (FTD) rapporteerden een hoge mate van emotionele belasting gerelateerd aan depressie en apathie in de patiënt (hoofdstuk 6). Verder bleek dat ontremming een belastend probleem was voor FTD mantelzorgers en angst belastend was voor AD mantelzorgers. Over het algemeen ervaarden FTD mantelzorgers een hogere emotionele belasting gerelateerd aan gedragsproblemen dan AD mantelzorgers. Concluderend hebben individuele gedragsproblemen een gedifferentieerde invloed op de mantelzorgers, waarbij stemmingsproblemen en apathie als meest belastend worden ervaren. Tevens blijkt er een verschil te zijn in emotionele belasting gerelateerd aan gedragsproblemen tussen specifieke diagnostische groepen, waarbij FTD mantelzorgers in het algemeen de grootste belasting ervaren door gedragsproblemen in vergelijking met AD mantelzorgers. Apathie had de meest negatieve invloed op de kwaliteit van de partnerrelatie, doordat een aantal essentiële kenmerken van de relatie hierdoor verloren ging, zoals het ondernemen van gezamenlijke activiteiten, het delen van ervaringen, en het verbaal communiceren. De resultaten geven aan dat mantelzorgers beter kunnen omgaan met actieve gedragingen die de interactie verstoren, zoals onrust en prikkelbaarheid, dan met een verminderde interactie met de patiënt veroorzaakt door apathie. 
Een hoge emotionele belasting in de mantelzorger heeft negatieve consequenties voor zowel patiënt als mantelzorger. De resultaten beschreven in hoofdstuk 7 laten zien dat de emotionele belasting gerelateerd aan gedragsproblemen een significante voorspeller is voor het moment van opname van de patient, terwijl gedragsproblemen op zichzelf geen voorspeller zijn. Deze resultaten geven weer dat het van belang is om objectieve stress (aanwezigheid van gedragsproblemen) te differentiëren van subjectieve belasting in de mantelzorger, wanneer men het risico op opname wilt bepalen. Tenslotte bleek ook de relatie met de patiënt een belangrijke factor te zijn, aangezien dochters eerder tot opname besloten dan partners van de dementerenden.

\section{De invloed van de mantelzorger op probleemgedrag bij dementie}

Naast het feit dat probleemgedrag van de patiënt ingrijpende gevolgen heeft voor de mantelzorger is het vice versa ook waarschijnlijk dat mantelzorgers invloed hebben op het ontstaan, in stand houden of verergeren van probleemgedrag van de patiënt. In het tweede deel van dit proefschrift werd ingegaan op de invloed van verschillende aspecten van het functioneren van de mantelzorger op probleemgedrag bij de patiënt (hoofdstuk 8. 9, 10 en 11). De resultaten uit deze studies geven aan dat lagere competenties van de mantelzorger om adequate zorg te geven geassocieerd zijn met een verhoogde mate van hyperactief gedrag bij de patiënt. Er zijn geen aanwijzingen dat stemming/apathie of psychose samenhangt met het functioneren van de mantelzorger.

Ten eerste werden in hoofdstuk 8 verschillen in zorgstrategiëen onderzocht aan de hand van interviews met de mantelzorger. Dit resulteerde in de identificatie van de volgende 3 strategiëen: (1) de eerste groep mantelzorgers hanteerde een "verzorgende" strategie en hadden het gevoel dat zij de zorg hadden voor een kind, zagen de dementerende niet meer als gelijkwaardig, en waren met name gericht op de persoonlijke verzorging en bescherming van de patiënt; (2) de tweede groep mantelzorgers hanteerde een "ondersteunende" strategie die werd gekenmerkt door aanpassing aan het niveau van functioneren van de patiënt, supervisie en stimulering van de dementerende; (3) de derde groep mantelzorgers hanteerde een "niet-adaptieve" strategie, die gekenmerkt werd door een gebrek aan acceptatie en begrip van de problemen, en een geïriteerde en ongeduldige houding ten opzichte van de patiënt. De drie groepen verschilden tevens in een aantal andere kenmerken van de mantelzorger, zoals geslacht, opleiding en persoonlijkheid. Vervolgens werden mogelijke gevolgen van deze zorgstrategiëen onderzocht. We vonden dat mantelzorgers met een niet- adaptieve strategie meer onrust rapporteerden bij de patiënt en zich minder competent voelden in de zorg voor de 
dementerende in vergelijking met de verzorgende en ondersteunende mantelzorgers. Daarentegen rapporteerden de ondersteunende mantelzorgers de minste onrust bij de patiënt en voelden zij zich het meest competent in hun rol als mantelzorger. Op basis van deze resultaten kunnen de meest kwetsbare patiënt-verzorger koppels worden geïdentificeerd, gekenmerkt door een niet-adaptieve zorgstrategie, gevoelens van verminderde competentie, en een hoge mate van hyperactieve symptomen in de patiënt. Het is zeer waarschijnlijk dat er sprake is van een wederzijdse negatieve interactie bij deze kwetsbare koppels, waarbij zowel de patiënt als de mantelzorger met irritatie en boosheid op elkaar reageren en zo een toename in hyperactiviteit bij de patiënt veroorzaken. Dit wordt verder ondersteund door de resultaten die beschreven worden in hoofdstuk 9, dat kritische en overbetrokken mantelzorgers, zoals bepaald met een maat voor Expressed Emotion (EE), een hogere mate van hyperactiviteit in de patiënt en verminderde gevoelens van competentie rapporteerden.

De kwetsbaarheid van patiënt-verzorger koppels wordt mogelijk ook vergroot door suboptimaal cognitief functioneren van de mantelzorger. De veeleisende zorgsituatie, die bovendien voortdurend veranderd, vereist optimaal cognitief functioneren van de mantelzorger. Echter partners van dementerenden zijn over het algemeen van dezelfde leeftijd als de patiënt en lopen daarom het risico op leeftijdsgerelateerde cognitieve achteruitgang (Jolles, 1986), hetgeen mogelijk een negatieve invloed heeft op de kwaliteit van de zorg voor de patiënt. De studie beschreven in hoofdstuk 10 naar het cognitief functioneren van mantelzorgers en mogelijke consequenties voor probleemgedrag van de patiënt laat zien dat suboptimaal cognitief functioneren van de mantelzorger gerelateerd is aan een toename in hyperactiviteit bij de patiënt. Deze bevinding sluit aan bij de resultaten beschreven in hoofdstuk 8 en 9 dat het functioneren van de mantelzorger geassocieerd is met hyperactiviteit bij de patiënt. Het feit dat suboptimaal cognitief functioneren van de mantelzorger een toename in hyperactiviteit veroorzaakte ondersteunt de hypothese dat het functioneren van de mantelzorger een rol speelt in de etiologie van probleemgedrag. Echter gezien de continue interactie tussen patiënt en mantelzorger is het het meest waarschijnlijk dat er een wederkerige relatie bestaat tussen probleemgedrag van de patiënt en het functioneren van de mantelzorger.

De resultaten van deze studie suggereren dat mantelzorgers die gekenmerkt worden door een niet-adaptieve strategie, hoge mate van Expressed Emotion of suboptimaal cognititief functioneren, mogelijk niet in staat zijn de verminderde competentie van de patiënt op te vangen en te compenseren. Daarentegen zijn mantelzorgers die 
gekenmerkt worden door een ondersteunende strategie beter in staat zich aan te passen aan de behoeftes van de patiënt en voor hem een veilige omgeving te creëren.

De hypothese dat het functioneren van de mantelzorger van invloed is op probleemgedrag van de dementerende wordt verder ondersteund door de resultaten van interventie studies. In hoofdstuk 11 wordt een literatuuroverzicht beschreven van studies naar interventies bij mantelzorgers van dementerenden, zoals gedragstherapeutische interventies, psycho-educatie, en sociale steun groepen. De resultaten van deze studies laten zien dat probleemgedrag bij de patiënt kan verminderen door het aanleren van adequate management strategiëen aan de mantelzorger. Dit onderstreept het belang van de resultaten van MAASBED, aangezien de identificatie van kwetsbare patient-verzorger koppels een belangrijk aangrijpingspunt zou kunnen zijn voor interventies gericht op verbetering van vaardigheden en strategieën van de mantelzorger om zo probleemgedrag van de patient te reduceren.

\section{Discussie en conclusie}

In hoofdstuk 12 worden de methodologie, bevindingen en hun relevantie besproken. Methodologische aandachtspunten zijn de sample selectie, meting van gedragsproblemen, uitval, de combinatie van kwalitatieve en kwanitatieve methodes, en de invloed van medicatiegebruik.

Probleemgedrag van de patiënt heeft negatieve consequenties voor het functioneren van de mantelzorger, waarbij apathie bij de patiënt een van de meest voorkomende en ingrijpende symptomen is. Verschillende verklaringen voor de rol van apathie worden bediscussieërd. De negatieve impact van apathie is een belangrijke bevinding omdat tot op heden de rol van apathie onderbelicht is gebleven in zowel de klinische praktijk als in interventie onderzoek. Verder geven de differentiële effecten van specifieke probleemgedragingen aan dat het belangrijk is om zowel in onderzoek als de praktijk verder te kijken dan gedragsproblemen in het algemeen naar individuele symptomen of syndromen.

Gezien het feit dat het functioneren van de mantelzorger, vice versa, ook van invloed lijkt op het probleemgedrag van de patiënt, wordt in hoofdstuk 12 het belang onderstreept van een interactief model van probleemgedrag bij dementie. Kwetsbare patiënt-verzorger systemen werden gekenmerkt door suboptimaal functioneren van de mantelzorger, verminderde gevoelens van competentie bij de mantelzorger, een negatieve interpersoonlijke interactie, en een hogere mate van hyperactiviteit bij de patiënt. Het 
identificeren van deze kwetsbare systemen vormt een belangrijk aangrijpingspunt voor de ontwikkeling van meer specifieke interventies.

Concluderend geven de resultaten van het onderzoek aan dat gedragsproblemen een belangrijk aspect zijn van dementie en daarom ook als een intrinsiek onderdeel van de ziekte moeten worden gezien. Een gedragsmatige benadering van dementie, met de nadruk op een interactief model van probleemgedrag, geeft goede mogelijkheden voor de ontwikkeling van succesvolle interventies gericht op het verbeteren van de kwaliteit van leven van zowel patiënt als mantelzorger. 
Samenvatting 


\section{References}


References

Aalten, P., Vugt, M. E., Lousberg, R., et al. Behavioral problems in dementia: a factor analysis of the Neuropsychiatric Inventory (NPI). Dement Geriatr Cogn Disord (2003) 15:99-105.

Acton, G. J., and Kang, J. Interventions to reduce the burden of caregiving for an adult with dementia: a metaanalysis. Research in Nursing and Health (2001) 24:349-360.

Alexopoulos, G. S., Abrams, R.C., Young, R.C., Shamoian, C.A. Cornell Scale for Depression in Dementia. Biological Psychiatry (1988) 23:271-284.

Alzheimer, A. Uber einen eigenartigen schweren Er Krankungsprozeb der Hirnrinde. Neurologisches Centralblatt (1906):1129-1136.

American Psychiatric Association, ed. Diagnostic and Statistical Manual of Mental Disorders: DSM-IV. American Psychiatric Association, 1994.

Arrindell, W. A., and Ettema, J. H. M. SCL-90. Handleiding bij een multidimensionele psychopathologie indicator. Swets \& Zeitlinger, 1986.

Ballard, C., O'Brien, J., James, I., et al. Dementia: management of behavioural and psychological symptoms. Oxford University Press, 2001.

Banerjee, S., Murray, J., Foley, B., et al. Predictors of institutionalisation in people with dementia. J Neurol Neurosurg Psychiatry (2003) 74(9):1315-6.

Bathgate, D., Snowden, J. S., Varma, A., et al. Behaviour in frontotemporal dementia, Alzheimer's disease and vascular dementia. Acta Neurol Scand (2001) 103(6):367-78.

Bauer, M. E., Vedhara, K., Perks, P., et al. Chronic stress in caregivers of dementia patients is associated with reduced lymphocyte sensitivity to glucocorticoids. Journal of neuroimmunology (2000) 103:84-92.

Baumgarten, M., Battista, R. N., Infante-Rivard, C., et al. The psychological and physical health of family members caring for an elderly person with dementia. Journal of clinical epidemiology (1992) 45(1):61-70.

Benoit, M., Dygai, I., Migneco, O., Robert, P. H., Bertogliati, C., Darcourt, J., Benoliel, J., Aubin-Brunet, V., Pringuey, D. Behavioral and psychological symptoms in Alzheimer's disease. Relation between apathy and regional cerebral perfusion. Dement Geriatr Cogn Disord (1999) 10(6):511-7.

Berkowitz, R., Shavit, N., and Leff, J. P. Educating relatives of schizophrenic patients. Soc Psychiatry Psychiatr Epidemiol (1990) 25(4):216-20.

Bianchetti, A., Zanetti, O., and Trabucchi, M. Non pharmacological treatment in Alzheimer's disease. Funct Neurol (1997) 12(3-4):215-7.

Bledin, K. D., MacCarthy, B., Kuipers, L., et al. Daughters of people with dementia. British journal of psychiatry (1990) 157:221-227.

Boucher, L., Renvall, M. J., and Jackson, J. E. Cognitively impaired spouses as primary caregivers for demented elderly people. J Am Geriatr Soc (1996) 44(7):828-31.

Bourgeois, M. S., Burgio, L. D., Schulz, R., et al. Modifying repetitive verbalizations of community-dwelling patients with AD. Gerontologist (1997) 37(1):30-9.

Bourgeois, M. S., Schulz, R., Burgio, L. D., et al. Skills training for spouses of patients with Alzheimer's disease: outcomes of an intervention study. Journal of Clinical Geropsychology (2002) 8(1):53-73.

Bozeat, S., Gregory, C. A., Ralph, M. A., et al. Which neuropsychiatric and behavioural features distinguish frontal and temporal variants of frontotemporal dementia from Alzheimer's disease? I Neurol Neurosurg Psychiatry (2000) 69(2):178-86.

Brand, N., and Jolles, J. Learning and retrieval rate of words presented auditorily and visually. J Gen Psychol (1985) 112(2):201-10.

Brandt, J., and Munro, C. A. Memory disorders in subcortical dementia. In A. D. Baddeley, M. D. Kopelman, and B. A. Wilson, eds., The handbook of memory disorders. John Wiley \& Sons Ltd, 2002.

Britten, N. Qualitative interviews in medical research. British Medical Journal (1995) 311:251-253. 
Brodaty, H. Psychosocial interventions in the Behavioural and Psychosocial Symptoms in Dementia (BPSD). International Psychogeriatrics (2003) 15(2).

Brodaty, H., Greene, A., and Koschera, A. Meta-analysis of psychosocial interventions for caregivers of people with dementia. JAGS (2003) 51:657-664.

Brodaty, H., and Luscombe, G. Psychological morbidity in caregivers is associated with depression in patients with dementia. Alzheimer Dis Assoc Disord (1998) 12(2):62-70.

Broderick, J. E., Arnold, D., Kudielka, B. M., et al. Salivary cortisol sampling compliance: comparison of patients and healthy volunteers. Psychoneuroendocrinology (2004) 29(5): 636-50.

Brown, S. A. Meta-analysis of diabetes patient education research: variations in intervention effects across studies. Research in Nursing and Health (1992) 15:409-419.

Burgener, S. C., Bakas, T., Murray, C., et al. Effective caregiver approaches for patients with Alzheimer's disease. Geriatr Nurs (1998) 19(3):121-6.

Butzlaff, R. L., Hooley, J.M. Expressed Emotion and Psychiatric Relapse. Archives of General Psychiatry (1998) 55:547552.

Cacioppo, J. T., Burleson, M. H., Poehlmann, K. M., et al. Autonomic and neuroendocrine responses to mild psychological stressors: effects of chronic stress on older women. The society of behavioral medicine (2000) 22(2):140-148.

Caswell, L. W., Vitaliano, P. P., Croyle, K. L., et al. Negative associations of chronic stress and cognitive performance in older adult spouse caregivers. Exp Aging Res (2003) 29(3):303-18.

Chan, D. C., Kasper, J. D., Black, B. S., et al. Presence of behavioral and psychological symptoms predicts nursing home placement in community-dwelling elders with cognitive impairment in univariate but not multivariate analysis. J Gerontol A Biol Sci Med Sci (2003) 58(6):548-54.

Chang, B. L. Cognitive-behavioral intervention for homebound caregivers of persons with dementia. Nurs Res (1999) 48(3):173-82.

Coen, R. F., Swanwick, G. R., O'Boyle, C. A., et al. Behaviour disturbance and other predictors of carer burden in Alzheimer's disease. Int J Geriatr Psychiatry (1997) 12(3):331-6.

Cohen, C. A., Gold, D. P., Shulman, K. I., et al. Factors determining the decision to institutionalize dementing individuals: a prospective study. Gerontologist (1993) 33(6):714-20.

Cohen, S., and Williamson, G. M. Perceived stress in a probability sample of the united states. In S. Spacepan and S. Oskamp, eds., The social psychology of Health. Sage Publications, 1988.

Coon, D. W., Thompson, L., Steffen, A., et al. Anger and depression management: psychoeducational skill training interventions for women caregivers of a relative with dementia. Gerontologist (2003) 43(5):678-89.

Corcoran, M. A. Gender differences in dementia management plans of spousal caregivers: implications for occupational therapy. Am J Occup Ther (1992) 46(11):1006-12.

Coste, J., Strauch, G., Letrath, M., et al. Reliability of hormonal levels for assessing the hypothalamic-pituitaryadrenocortical system in clinical pharmacology. Br J Clin Pharmacol (1994) 42(1):63-68.

Cummings, J. L. The Neuropsychiatric Inventory: assessing psychopathology in dementia patients. Neurology (1997) 48(5 Suppl 6):S10-6.

Cummings, J. L. Cholinesterase inhibitors: A new class of psychotropic compounds. Am J Psychiatry (2000) 157(1):415.

Cummings, J. L., and McPherson, S. Neuropsychiatric assessment of Alzheimer's disease and related dementias. Aging (Milano) (2001) 13(3):240-6.

Cummings, J. L., Mega, M., Gray, K., Rosenberg-Thompson, S., Carusi, D.A., Gornbein, J. The Neuropsychiatric Inventory: Comprehensive assessment of psychopathology in dementia. Neurology (1994) 44:2308-2314. 
References

Cummings, J. L., Victoroff, J.I. Noncognitive Neuropsychiatric Syndromes in Alzheimer's Disease. Neuropsychiatry, Neuropsychology, and Behavioral Neurology (1990) 2:140-158.

Da Roza Davis, J. M., and Cowen, P. J. Biochemical stress of caring. Psychological Medicine (2001) 31:1475-1478.

De Deyn, P. P. The management of behavioural and psychotic disturbances in the elderly. Clinician (1998) 16(1):4355.

De Vet, H. C. W., de Bie, R. A., G.J.M.G., v. d. H., et al. Systematic reviews on the basis of methodological criteria. Physiotherapy (1997) 83(6):284-289.

Deimling, G. T., and Bass, D. M. Symptoms of mental impairment among elderly adults and their effects on family caregivers. J Gerontol (1986) 41(6):778-84.

Derix, M. M., Hofstede, A. B., Teunisse, S., et al. [CAMDEX-N: the Dutch version of the Cambridge Examination for Mental Disorders of the Elderly with automatic data processing]. Tijdschrift voor Gerontologie en Geriatrie (1991) 22(4):143-50.

Devanand, D. P., Brockington, C. D., Moody, B. J., Brown, R. P., Mayeux, R., Endicott, J., Sackeim, H. A. Behavioral syndromes in Alzheimer's disease. Int Psychogeriatr (1992a) 4(Suppl 2):161-84.

Devanand, D. P., Miller, L., Richards, M., Marder, K., Bell, K., Mayeux, R, Stern, Y. The Columbia University Scale for psychopathology in Alzheimer's disease. Archives of Neurology (1992b) 49:371-376.

Diehl, J., and Kurz, A. Frontotemporal dementia: patient characteristics, cognition, and behaviour. Int J Geriatr Psychiatry (2002) 17(10):914-8.

Donaldson, C., Tarrier, N., and Burns, A. The impact of the symptoms of dementia on caregivers [see comments]. Br J Psychiatry (1997) 170:62-8.

Donaldson, C., Tarrier, N., and Burns, A. Determinants of carer stress in Alzheimer's disease. Int J Geriatr Psychiatry (1998) 13(4):248-56.

Done, D. J., and Thomas, J. A. Training in communication skills for informal carers of people suffering from dementia: a cluster randomized clinical trial comparing a therapist led workshop and a booklet. Int J Geriatr Psychiatry (2001) 16(8):816-21.

Draper, B. M., Poulos, R. G., Poulos, C. J., et al. Risk factors for stress in elderly caregivers. International journal of geriatric psychiatry (1995) 11:227-231.

Dura, J. R., and Kiecolt-Glaser, J. K. Sample bias in caregiving research. J Gerontol (1990) 45(5):200-4.

Ebrecht, M., Hextall, J., Kirtley, L. G., et al. Perceived stress and cortisol levels predict speed of wound healing in healthy male adults. Psychoneuroendocrinology (2003) 29(6): 798-809.

Eriksson, S. impact of the environment on behavioral and psychological symptoms of dementia. international psychogeriatrics (2000) 12:89-91.

Eriksson, S., Minthon, L., K.M., M., et al. BPSD from a Nordic perspective; Behavioural and psychological symptoms in dementia. A state-of-the-art document. Janssen-Cilag AB, 2000.

Fearon, M., Donaldson, C., Burns, A., et al. Intimacy as a determinant of expressed emotion in carers of people with Alzheimer's disease. Psychol Med (1998) 28(5):1085-90.

Finkel, S. The significance of the behavioural and psychological symptoms of dementia. Clinician (1998) 16(1):33-42.

Finkel, S. I., Costa e Silva, J., Cohen, G., et al. Behavioral and psychological signs and symptoms of dementia: a consensus statement on current knowledge and implications for research and treatment. Int Psychogeriatr (1996) 8(Suppl 3):497-500.

Fisher, L., and Lieberman, M. A. A longitudinal study of predictors of nursing home placement for patients with dementia: the contribution of family characteristics. Gerontologist (1999) 39(6):677-86.

Fitting, M., Rabins, P., Lucas, M. J., et al. Caregivers for dementia patients: a comparison of husbands and wives. Gerontologist (1986) 26(3):248-52. 
Folstein, M. F., Folstein, S. E., and McHugh, P. R. "Mini-Mental State": A practical method for grading the cognitive state of patients for the clinician. Journal of Psychiatric Research (1975) 12:189-198.

Forstl, H. Neuropathology of Behavioural and Psychological Symptoms of dementia. Int Psychogeriatr (2000) 12(suppl 1):77-81.

Frisoni, G. B., Rozzini, L., Gozzetti, A., et al. Behavioral syndromes in Alzheimer's disease: description and correlates. Dement Geriatr Cogn Disord (1999) 10(2):130-8.

Fuh, J. L., Liu, C. K., Mega, M. S., et al. Behavioral disorders and caregivers' reaction in Taiwanese patients with Alzheimer's disease. Int Psychogeriatr (2001) 13(1):121-8.

Gallagher-Thompson, D., Dal Canto, P. G., Jacob, T., et al. A comparison of marital interaction patterns between couples in which the husband does or does not have Alzheimer's disease. Journal of Gerontology: social sciences (2001) 56B(3):140-150.

Gallicchio, L., Siddiqi, N., Langenberg, P., et al. Gender differences in burden and depression among informal caregivers of demented elders in the community. Int J Geriatr Psychiatry (2002) 17(2):154-63.

Gaugler, J. E., Davey, A., Pearlin, L. I., et al. Modeling caregiver adaptation over time: the longitudinal impact of behavior problems. Psychol Aging (2000a) 15(3):437-50.

Gaugler, J. E., Edwards, A. B., Femia, E. E., et al. Predictors of institutionalization of cognitively impaired elders: family help and the timing of placement. J Gerontol B Psychol Sci Soc Sci (2000b) 55(4):P247-55.

Gaugler, J. E., Kane, R. L., Kane, R. A., et al. Caregiving and institutionalization of cognitively impaired older people: utilizing dynamic predictors of change. Gerontologist (2003) 43(2):219-29.

Gendron, C., Poitras, L., Dastoor, D. P., et al. Cognitive-behavioural group intervention for spousal caregivers: findings and clinical considerations. Clinical Gerontologist (1996) 17(1):3-19.

Gerdner, L. A., Buckwalter, K. C., and Reed, D. Impact of a psychoeducational intervention on caregiver response to behavioural problems. Nurs Res (2002) 51(6):363-74.

Gerdner, L. A., Hall, G. R., and Buckwalter, K. C. Caregiver training for people with Alzheimer's based on a stress threshold model. Image J Nurs Sch (1996) 28(3):241-6.

Gilhooly, M. L., and Whittick, J. E. Expressed emotion in caregivers of the dementing elderly. Br J Med Psychol (1989) 62(Pt 3):265-72.

Gitlin, L. N., Corcoran, M., Winter, L., et al. A randomized, controlled trial of a home environmental intervention: effect on efficacy and upset in caregivers and on daily function of persons with dementia. Gerontologist (2001) 41(1):414.

Gitlin, L. N., Winter, L., Dennis, M. P., et al. Strategies used by families to simplify tasks for individuals with Alzheimer's disease and related disorders: psychometric analysis of the Task Management Strategy Index (TMSI). Gerontologist (2002) 42(1):61-9.

Gonzalez-Salvador, M. T., Arango, C., Lyketsos, C. G., et al. The stress and psychological morbidity of the Alzheimer patient caregiver. Int J Geriatr Psychiatry (1999) 14(9):701-710.

Gormley, N., Lyons, D., and Howard, R. Behavioural management of aggression in dementia: a randomized controlled trial. Age Ageing (2001) 30(2):141-5.

Greene, J. G., Smith, R., Gardiner, M., et al. Measuring behavioural disturbance of elderly demented patients in the community and its effects on relatives: a factor analytic study. Age Ageing (1982) 11(2):121-6.

Gunnar, M. R., Morison, S. J., Chisholm, K., et al. Salivary cortisol levels in children adopted from romanian orphanages. Dev Psychopathol (2001) 13(3):611-28.

Hair, Anderson, Tatham, et al. Multivariate data analysis, fifth edition. Prentice Hall, 1988.

Hamel, M., Gold, D. P., Andres, D., et al. Predictors and consequences of aggressive behavior by community-based dementia patients. Gerontologist (1990) 30(2):206-11.

Hamilton, M. A. A rating scale for depression. Journal of Neurology, Neurosurgery and Psychiatry (1960) 23:56-62. 
References

Harvath, T. A. Interpretation and management of dementia-related behavior problems. Clin Nurs Res (1994) 3(1):7-25; discussion 25-6.

Haupt, M., Karger, A., and Janner, M. Improvement of agitation and anxiety in demented patients after psychoeducative group intervention with their caregivers. Int J Geriatr Psychiatry (2000) 15(12):1125-1129.

Hebert, R., Dubois, M. F., Wolfson, C., et al. Factors associated with long-term institutionalization of older people with dementia: data from the Canadian Study of Health and Aging. J Gerontol A Biol Sci Med Sci (2001) 56(11):M693-9.

Hebert, R., Leclerc, G., Bravo, G., et al. Efficacy of a support group programme for caregivers of demented patients in the community: a randomized controlled trial. Archives of Gerontology and Geriatrics (1994) 18:1-4.

Hebert, R., Levesque, L., Vezina, J., et al. Efficacy of a psychoeducative group program for caregivers of demented persons living at home: a randomized controlled trial. J Gerontol B Psychol Sci Soc Sci (2003) 58(1):S58-67.

Hinrichsen, G. A., and Niederehe, G. Dementia management strategies and adjustment of family members of older patients. Gerontologist (1994) 34(1):95-102.

Honig, A., Hofman, A., Rozendaal, N., et al. Psycho-education in bipolar disorder: effect on expressed emotion. Psychiatry Res (1997) 72(1):17-22.

Hooker, K., Bowman, S. R., Coehlo, D. P., et al. Behavioral change in persons with dementia: relationships with mental and physical health of caregivers. J Gerontol B Psychol Sci Soc Sci (2002) 57(5):P453-60.

Hooley, J. M., Orley, J., and Teasdale, J. D. Levels of expressed emotion and relapse in depressed patients. Br J Psychiatry (1986) 148:642-7.

Hope, T., Keene, J., Gedling, K., et al. Predictors of institutionalization for people with dementia living at home with a carer. Int J Geriatr Psychiatry (1998) 13(10):682-90.

Hope, T., Keene, J., Fairburn, C., McShane, R., Jacoby, R. Behaviour changes in dementia. 2: Are there behavioural syndromes? Int J Geriatr Psychiatry (1997) 12(11):1074-8.

Horowitz, A., and Shindelman, L. W. Reciprocity and affection: past influences on current caregiving. Journal of gerontological social work (1983) 5:5-20.

Houx, P. J., Shepherd, J., Blauw, G. J., et al. Testing cognitive function in elderly populations: the PROSPER study. PROspective Study of Pravastatin in the Elderly at Risk. J Neurol Neurosurg Psychiatry (2002) 73(4):385-9.

Huang, H. L., Shyu, Y. I., Chen, M. C., et al. A pilot study on a home-based caregiver training program for improving caregiver self-efficacy and decreasing the behavioral problems of elders with dementia in Taiwan. Int J Geriatr Psychiatry (2003) 18(4):337-45.

IPA. Behavioural and Psychological Symptoms of Dementia (BPSD). Educational Pack. International Psychogeriatric Association, 2003.

Irwin, M., Hauger, R., Patterson, T. L., et al. Alzheimer caregiver stress: basal natural killer cell activity, pituitary-adrenal cortical function, and sympathetic tone. Ann Behav Med (1997) 19(2):83-90.

Jolles, J. Cognitive, emotional and behavioral dysfunctions in aging and dementia. Prog Brain Res (1986) 70:15-39.

Jolles, J., Houx, P. J., Boxtel van, M. P. J., et al. The Maastricht Aging Study: determinants of cognitive aging. Neuropsych Publishers, 1995.

Kat, M. G., De Jonghe, J. F. M., Aalten, P., et al. Neuropsychiatrische symptomen bij dementie: psychometrische aspecten van de Nederlandse Neuropsychiatric Inventory (NPI). Tijdschrift voor Gerontologie en Geriatrie (2002) 33:150-155.

Katon, W., Kleinman, A., and Rosen, G. Depression and somatization: a review. Am J Med (1982) 72:127-135.

Kaufer, D., Cummings, J. L., and Christine, D. Differential neuropsychiatric symptom responses to tacrine in Alzheimer's disease: relationship to dementia severity. J Neuropsychiatry Clin Neurosci (1998) 10(1):55-63.

Kaufer, D. I., Cummings, J.L., Christine, D., Bray, T., Castellon, S., Masterman, D., Macmillan, A., Ketchel, P., DeKosky, S.T. Assessing the impact of neuropsychiatric symptoms in Alzheimer's Disease: The Neuropsychiatric Inventory Caregiver Distress Scale. Journal of American Geriatrics Society (1998) 46:2109-215. 
Kiecolt-Glaser, J. K., Dura, J. R., Speicher, C. E., et al. Spousal caregivers of dementia victims: longitudinal changes in immunity and health. Psychosom Med (1991) 53:345-362.

Kim, J. M., Shin, I. S., Jeong, S. J., et al. Predictors of institutionalization in patients with dementia in Korea. Int J Geriatr Psychiatry (2002) 17(2):101-6.

Kirschbaum, C., and Hellhamer, D. H. Salivary cortisol in psychoneuroendocrine research: Recent developments and applications. Psychoneuroendocrinology (1994) 19(4):313-333.

Knopman, D. S., Berg, J. D., Thomas, R., et al. Nursing home placement is related to dementia progression: experience from a clinical trial. Alzheimer's Disease Cooperative Study. Neurology (1999) 52(4):714-8.

Knopman, D. S., Kitto, J., Deinard, S., et al. Longitudinal study of death and institutionalization in patients with primary degenerative dementia. J Am Geriatr Soc (1988) 36(2):108-12.

Kudielka, B. M., Broderick, J. E., and Kirschbaum, C. Compliance with saliva sampling protocols: electronic monitoring reveals invalid cortisol daytime profiles in noncompliant subjects. Psychosom Med (2003) 65(2):313-9.

Kudielka, B. M., and Kirschbaum, C. Awakening cortisol responses are influenced by health status and awakening time but not by menstrual cycle phase. Psychoneuroendocrinology (2003) 28(1):35-47.

Kuhn, D. R., and Mendes de Leon, C. F. Evaluating an educational intervention with relatives of persons in the early stages of Alzheimer's disease. Research on social work practice (2001) 11(5):531-548.

Kunz-Ebrecht, S. R., Kirschbaum, C., Marmot, M., et al. Differences in cortisol awakening response on work days and weekends in women and men from the Whitehall II cohort. Psychoendocrinology (2004) 29 (4): 516-528.

Landes, A. M., Sperry, S. D., Strauss, M. E., et al. Apathy in Alzheimer's Disease. Jounal of American Geriatrics Society (2001) 49:1700-1707.

Lawlor, B., and Ni Bhriain, S. Psychosis and behavioural symptoms of dementia: defining the role of neuroleptic interventions. Int J Geriatr Psychiatry (2001) 16 Suppl 1:S2-6.

Lawlor, B. A. Environmental and social aspects of behavioral disturbances in dementia. International Psychogeriatrics (1996) 8(3):259-261.

Lawrence, R. H., Tennstedt, S. L., and Assmann, S. F. Quality of the caregiver--care recipient relationship: does it offset negative consequences of caregiving for family caregivers? Psychol Aging (1998) 13(1):150-8.

Lawton, M. P. Competence, environmental press and adaptation. In P. G. Windley, T. O. Byerts, and F. G. Ernst, eds., Theory development in environment and ageing. Gerontological Society, 1975.

Leff, J., and Vaughn, C. Experimental emotion in families: its significance for mental illness. Guilford Press, 1985.

Levy, M. L., Miller, B. L., Cummings, J. L., et al. Alzheimer disease and frontotemporal dementias. Behavioral distinctions [see comments]. Arch Neurol (1996) 53(7):687-90.

Lieberman, M. A., and Kramer, J. H. Factors affecting decisions to institutionalize demented elderly. Gerontologist (1991) 31(3):371-4.

Lindgren, C. L. The caregiver career. Image J Nurs Sch (1993) 25(3):214-9.

LoGiudice, D., Waltrowicz, W., McKenzie, S., et al. Prevalence of dementia among patients referred to an aged care assessment team and associated stress in their carers. Australian journal of public health (1995) 19:275-279.

Lopez, O. L., Wisniewski, S. R., Becker, J. T., et al. Psychiatric medication and abnormal behavior as predictors of progression in probable Alzheimer disease. Arch Neurol (1999) 56(10):1266-72.

Lupien, S., Lecours, A. R., Lussier, I., et al. Basal cortisol levels and cognitive deficits in human aging. J Neurosci (1994) 14(5 Pt 1):2893-903.

Lupien, S. J., de Leon, M., de Santi, S., et al. Cortisol levels during human aging predict hippocampal atrophy and memory deficits. Nat Neurosci (1998) 1(1):69-73.

Luteijn, F., and van der Ploeg, F. A. E. Handleiding Groningen Intelligentie Test (GIT) [Manual Groningen Intelligence Test]. Swets \& Zeitlinger, 1983. 
References

Lyketsos, C. G., Breitner, J. C., and Rabins, P. V. An evidence-based proposal for the classification of neuropsychiatric disturbance in Alzheimer's disease. Int J Geriatr Psychiatry (2001a) 16(11):1037-42.

Lyketsos, C. G., Sheppard, J. M., Steinberg, M., et al. Neuropsychiatric disturbance in Alzheimer's disease clusters into three groups: the Cache County study. Int J Geriatr Psychiatry (2001b) 16(11):1043-53.

Magana, A. B., Goldstein, J. M., Karno, M., et al. A brief method for assessing expressed emotion in relatives of psychiatric patients. Psychiatry Res (1986) 17(3):203-12.

Marchello, V., Boczko, F., and Shelkey, M. Progressive dementia: strategies to manage new problem behaviors. Geriatrics (1995) 50(3):40-3; quiz 44-5.

Marriott, A., Donaldson, C., Tarrier, N., et al. Effectiveness of cognitive-behavioural family intervention in reducing the burden of care in carers of patients with Alzheimer's disease. Br J Psychiatry (2000) 176:557-62.

Matsuda, O. The effect of coping on the caregiver of elderly patients with dementia [published erratum appears in Psychiatry Clin Neurosci 1995 Dec;49(5-6):308]. Psychiatry Clin Neurosci (1995) 49(4):209-11.

McEwen, B. S., and Sapolsky, R. M. Stress and cognitive function. Curr Opin Neurobiol (1995) 5(2):205-16.

McKeith, I., Galasko, D., Kosaka, K., et al. Consensus guidelines for the clinical and pathologic diagnosis of dementia with Lewy bodies (DLB): report of the consortium on DLB international workshop. Neurology (1996) 47(5):1113-24.

McKhann, G., Drachman, D., Folstein, M. F., et al. Clinical diagnosis of Alzheimer's disease: report of the NINCDS-ADRA Work group under the auspices of Department of Health and Human Services Task Force on Alzheimer's Disease. Neurology (1984) 34(7):939-44.

McShane, R. What are the syndromes of behavioral and psychological symptoms of dementia? International Psychogeriatrics (2000) 12:147-153.

Mega, M. S., Cummings, J.L., Fiorello, T., Gornbein, J. The spectrum of behavioral changes in Alzheimer's disease. Neurology (1996) 46:130-135.

Mega, M. S., Dinov, I. D., Lee, L., O'Connor, S. M., Masterman, D. M., Wilen, B., Mishkin, F., Toga, A. W., Cummings, J. L. Orbital and dorsolateral frontal perfusion defect associated with behavioral response to cholinesterase inhibitor therapy in Alzheimer's disease. J Neuropsychiatry Clin Neurosci (2000) 12(2):209-18.

Meins, W., Frey, A., and Thiesemann, R. Premorbid personality traits in Alzheimer's disease: do they predispose to noncognitive behavioral symptoms? [In Process Citation]. International Psychogeriatrics (1998) 10(4):369-78.

Mills, P. J., Ziegler, M. G., Patterson, T., et al. Plasma catecholamine and lymphocyte beta-2-adrenergic receptor alterations in elderly Alzheimer caregivers under stress. Psychosomatic Medicine (1997) 59:251-256.

Montgomery, S., and Asberg, M. A new depression scale designed to be sensitive to change. British Journal of Psychiatry (1979) 134:382-389.

Morris, L. W., Morris, R. G., and Britton, P. G. The relationship between marital intimacy, perceived strain and depression in spouse caregivers of dementia sufferers. British journal of medical psychychology (1988) 61:231-236.

Murray, J., Schneider, J., Banerjee, S., et al. EUROCARE: a cross-national study of co-resident spouse carers for people with Alzheimer's disease: II--A qualitative analysis of the experience of caregiving. Int J Geriatr Psychiatry (1999) 14(8):662-7.

Nicolson, N. A., and van Diest, R. Salivary cortisol patterns in vital exhaustion. J Psychosom Res (2000) 49(5):335-42.

O'Donell, B., Drachman, D., Barnes, H., et al. Incontinence and troublesome behaviors predict institutionalization in dementia. J Geriatric Psychiatry and Neurology (1992) 5:45-52.

Ostwald, S. K., Hepburn, K. W., Caron, W., et al. Reducing caregiver burden: a randomized psychoeducational intervention for caregivers of persons with dementia. Gerontologist (1999) 39(3):299-309.

Parker, G., and Hadzi-Pavlovic, D. Expressed emotion as a predictor of schizophrenic relapse: an analysis of aggregated data. Psychological medicine (1990) 20:961-965.

Parmelee, P. A., and Lawton, M. P. The design of special environments for the aged. In J. E. Birren and K. W. Schaie, eds., Handbook of the psychology of aging. Academic Press, 1990. 
Patterson, G. R., and Hops, H. Coercion, a game for two: Intervention techniques for marital conflict. In R. E. Ulrich and P. Mountjoy, eds., The experimental analysis of social behavior. Appleton-Century-Crofts, 1972.

Perry, R. J., and Miller, B. L. Behavior and treatment in frontotemporal dementia. Neurology (2001) 56(4):S46-s51.

Phillips, V. L., and Diwan, S. The incremental effect of dementia-related problem behaviors on the time to nursing home placement in poor, frail, demented older people. J Am Geriatr Soc (2003) 51(2):188-93.

Pinquart, M., and Sorensen, S. Associations of stressors and uplifts of caregiving with caregiver burden and depressive mood: a meta-analysis. J Gerontol B Psychol Sci Soc Sci (2003) 58(2):P112-28.

Pope, C., and Mays, N. Reaching the parts other methods cannot reach: an introduction to qualitative methods in health and health services research. British Medical Journal (1995) 311(42-45).

Pot, A. M. Caregiver perspectives: a longitudinal study on the psychological distress of informal caregivers of demented elderly, Department of Psychiatry. Vrije Universiteit, 1996.

Powers, D. V., Gallagher-Thompson, D., and Kraemer, H. C. Coping and depression in Alzheimer's caregivers: longitudinal evidence of stability. J Gerontol B Psychol Sci Soc Sci (2002) 57(3):P205-11.

Preussner, J., and Hellhamer, D. H. Burnout, perceived stress, and cortisol reponses to awakening. Psychosomatic medicine (1999) 61:197-204.

Preussner, J., Wolf, O. T., and Hellhamer, D. H. Free cortisol levels after awakening: a reliable biological marker for the assessment for adrenocortical activity. Life Sci (1997) 61(26):2539-49.

Preussner, M., Hellhamer, D. H., J.C., P., et al. Self-reported depressive symptoms and stress levels in healthy young men: associations with the cortisol response to awakening. Psychosom Med (2003) 65(1):92-99.

Pruchno, R. A., Michaels, J. E., and Potashnik, S. L. Predictors of institutionalization among Alzheimer disease victims with caregiving spouses. Journal of Gerontology (1990) 45(6):S259-66.

Pruchno, R. A., and Resch, N. L. Aberrant behaviors and Alzheimer's disease: mental health effects on spouse caregivers. J Gerontol (1989) 44(5):S177-82.

Quayhagen, M. P., Quayhagen, M., Corbeil, R. R., et al. Coping with dementia: evaluation of four nonpharmacologic interventions. Int Psychogeriatr (2000) 12(2):249-65.

Reisberg, B., Ferris, S. H., de Leon, M. J., et al. The Global Deterioration Scale for assessment of primary degenerative dementia. Am J Psychiatry (1982) 139(9):1136-9.

Riello, R., Geroldi, C., Parrinello, G., et al. The relationship between biological and environmental determinants of delusions in mild Alzheimer's disease patients. Int J Geriatr Psychiatry (2002) 17(7):687-8.

Ritchie, J., and Spencer, L. Qualitative data analysis for applied policy research. In A. Bryman and P. Burgess, eds., Analysing qualitative data. Sage, 1994.

Riviere, S., Gillette-Guyonnet, S., Andrieu, S., et al. Cognitive function and caregiver burden: predictive factors for eating behaviour disorders in Alzheimer's disease. Int J Geriatr Psychiatry (2002) 17(10):950-5.

Roberto, K. A., Richter, J. M., Bottenberg, D. J., et al. Communication patterns between caregivers and their spouses with Alzheimer's disease: a case study. Arch Psychiatr Nurs (1998) 12(4):202-8.

Robinson, K. M., Adkisson, P., and Weinrich, S. Problem behaviour, caregiver reactions, and impact among caregivers of persons with Alzheimer's disease. J Adv Nurs (2001) 36(4):573-82.

Roman, G., Tatemichi, T., Erkinjuntti, T., et al. Vascular dementia: diagnostic criteria for research studies. Report of the NINDS-AIREN International Workshop. Neurology (1993) 43(2):250-60.

Rosso, S. M., Roks, G., Stevens, M., et al. Complex compulsive behaviour in the temporal variant of frontotemporal dementia. J Neurol (2001) 248(11):965-70.

Rymer, S., Salloway, S., Norton, L., et al. Impaired awareness, behavior disturbance, and caregiver burden in Alzheimer disease. Alzheimer Dis Assoc Disord (2002) 16(4):248-53.

Sapolsky, R. M., Krey, L. C., and McEwen, B. S. The neuroendocrinology of stress and aging: the glucocorticoid cascade hypothesis. Endocr Rev (1986) 7(3):284-301. 


\section{References}

Schmidt-Reinwald, A., Pruessner, J. C., Hellhammer, D. H., et al. The cortisol response to awakening in relation to different challenge tests and a 12-hour cortisol rhythm. Life Sci (1999) 64(18):1653-60.

Schulz, P., Kirschbaum, C., Preussner, J., et al. Increased free cortisol secretion after awakening in chronically stressed individuals due to work overload. Stress Medicine (1998) 14:91-97.

Schulz, R., O'Brien, A., Czaja, S., et al. Dementia caregiver intervention research: in search of clinical significance. Gerontologist (2002) 42(5):589-602.

Schulz, R., O'Brien, A. T., Bookwala, J., et al. Psychiatric and physical morbidity effects of dementia caregiving: Prevalence, correlates, and causes. The Gerontological Society of America (1995) 35:771-791.

Seidel, J. The Ethnograph v5.0: a user's guide. Qualis Research Associates, 1998.

Severson, M. A., Smith, G. E., Tangalos, E. G., et al. Patterns and predictors of institutionalization in community-based dementia patients. Journal of American Geriatrics Society (1994) 42:181-185.

Smith, A. The Symbol Digit Modalities Test: a neuropsychological test for economic screening of learning and other cerebral disorders. Learning disorders (1968) 36:83-91.

Snowden, J. S., Bathgate, D., Varma, A., et al. Distinct behavioural profiles in frontotemporal dementia and semantic dementia. J Neurol Neurosurg Psychiatry (2001) 70(3):323-32.

Spruytte, N., Van Audenhove, C., and Lammertyn, F. Predictors of institutionalization of cognitively-impaired elderly cared for by their relatives. Int J Geriatr Psychiatry (2001) 16(12):1119-28.

Stewart, J. T. Management of behavior problems in the demented patient. Am Fam Physician (1995) 52(8):2311-7, $2321-2$.

Strauss, M. E., Lee, M.M., DiFilippo, J.M. Premorbid personality and behavioral symptoms in Alzheimer Disease. Archives of Neurology (1997) 54:257-259

Street, J. S., Clark, W. S., Gannon, K. S., et al. Olanzapine treatment of psychotic and behavioral symptoms in patients with Alzheimer disease in nursing care facilities: a double-blind, randomized, placebo-controlled trial. The HGEU Study Group. Arch Gen Psychiatry (2000) 57(10):968-76.

Stroop, J. R. Studies of interference in serial verbal reactions. Journal of experimental psychology (1935) 18:643-662.

Sultzer, D. L. Neuroimaging and the origin of psychiatric symptoms in dementia. Int Psychogeriatr (1996) 8(Suppl 3):239-43; discussion 269-72.

Sultzer, D. L., Mahler, M. E., Mandelkern, M. A., et al. The relationship between psychiatric symptoms and regional cortical metabolism in Alzheimer's disease. Journal of Neuropsychiatry and Clinical Neurosciences (1995) 7(4):476-84.

Sultzer, D. L., Mahler, M. E., Cummings, J. L., Van Gorp, W. G., Hinkin, C. H., Brown, C. Cortical abnormalities associated with subcortical lesions in vascular dementia. Clinical and position emission tomographic findings. Arch Neurol (1995) 52(8):773-80

Tariot, P. N., Mack, J. L., Patterson, M. B., et al. The Behavior Rating Scale for dementia of the consortium to establish a registry for Alzheimer's disease. American Journal of Psychiatry (1995) 152(9):1349-1357.

Tarrier, N., Barrowclough, C., Ward, J., et al. Expressed emotion and attributions in the carers of patients with Alzheimer's disease: the effect on carer burden. J Abnorm Psychol (2002) 111(2):340-9.

Teri, L. Training families to provide care: effects on people with dementia. Int J Geriatr Psychiatry (1999) 14(2):110-6; discussion 116-9.

Teri, L., Logsdon, R. G., and McCurry, S. M. Nonpharmacologic treatment of behavioral disturbance in dementia. The Medical Clinics of North America (2002) 86:641-656.

Teri, L., Logsdon, R. G., Peskind, E., et al. Treatment of agitation in AD: a randomized, placebo-controlled clinical trial. Neurology (2000) 55(9):1271-8.

Teri, L., Logsdon, R. G., Uomoto, J., et al. Behavioral treatment of depression in dementia patients: a controlled clinical trial. J Gerontol B Psychol Sci Soc Sci (1997) 52(4):P159-66. 
Teri, L., Logsdon, R. G., Whall, A. L., et al. Treatment for agitation in dementia patients: a behaviour managment approach. Psychotherapy (1998) 35(4):436-443.

Teri, L., and Uomoto, J. Reducing excess disability in dementia patients:training caregivers to manage patient depression. Clinical Gerontologist (1991) 10(4):49-63.

Teunisse, S. Activities of daily living scales in dementia: their development and future, Developments in dementia and functional disorders in the elderly. Wrightson Biomedical Publishing Ltd, 1995.

Teunisse, S., and Derix, M. M. A. Measuring functional disability in community dwelling dementia patients: development of a questionnaire. Tijdschrift voor Gerontologie en Geriatrie (1991) 22:53-59.

The Lund and Manchester groups. Clinical and neuropathological criteria for frontotemporal dementia. J Neurol Neurosurg Psychiatry (1994) 57:416-418.

Van der Zee, K. I., and Sanderman, R. Het meten van de algemene gezondheidstoestand met de RAND-36: een handleiding [Measurement of general health with the RAND-36: a manual]. Noordelijk Centrum voor Gezondheidsvraagstukken NCG, 1993.

Vedhara, K., Cox, N. K. M., Wilcock, G. K., et al. Chronic stress in elderly carers of dementia patients and antibody response to influenza vaccination. The Lancet (1999) 353:627-631.

Vernooij-Dassen, M. J., Persoon, J. M., and Felling, A. J. Predictors of sense of competence in caregivers of demented persons. Soc Sci Med (1996) 43(1):41-9.

Vernooij-Dassen, M. J. F. J., Felling, A. J. A., Brummelkamp, E., et al. Assessment of caregiver's competence in dealing with the burden of caregiving for a dementia patient: a short sense of competence questionnaire (SSCQ) suitable for clinical practice. American Geriatrics Society (1999) 47:256-262.

Victoroff, J., Mack, W. J., and Nielson, K. A. Psychiatric complications of dementia: impact on caregivers. Dement Geriatr Cogn Disord (1998) 9(1):50-5.

Vitaliano, P. P., Young, H. M., Russo, J., et al. Does expressed emotion in spouses predict subsequent problems among care recipients with Alzheimer's disease? Journal of gerontology: psychological sciences (1993) 48(4):202209.

Vugt de, M. E., Nicolson, N. A., Aalten, P., et al. Behavioral problems in dementia and salivary cortisol patterns in caregivers. J Neurology and Clinical Neuroscience (2004, in press).

Vugt, d. M. E., Stevens, F., Aalten, P., et al. Do caregiver management strategies influence patient behaviour in dementia? Int J Geriatr Psychiatry (2004) 19:85-92.

Vugt, M. E., Stevens, F., Aalten, P., et al. Behavioural disturbances in dementia patients and quality of the marital relationship. Int J Geriatr Psychiatry (2003) 18:149-154.

Wagner, A. W., Logsdon, R. G., Pearson, J. L., et al. Caregiver expressed emotion and depression in Alzheimer's disease. Aging \& Mental Health (1997) 1(2):132-139.

Williamson, G. M., and Shaffer, D. R. Relationship quality and potentially harmful behaviors by spousal caregivers: how we were then, how we are now. The Family Relationships in Late Life Project. Psychol Aging (2001) 16(2):21726.

Wright, L. K. The impact of Alzheimer's disease on the marital relationship. Gerontologist (1991) 31(2):224-37.

Wust, S., Federenko, I., Hellhammer, D. H., et al. Genetic factors, perceived chronic stress, and the free cortisol response to awakening. Psychoneuroendocrinology (2000a) 25(7):707-20.

Wust, S., Wolf, J., Hellhammer, D. H., et al. the cortisol awakening response - normal values and confounds. Noise and Health (2000b) 7:77-85.

Yaffe, K., Fox, P., Newcomer, R., et al. Patient and caregiver characteristics and nursing home placement in patients with dementia. Jama (2002) 287(16):2090-7.

Yehuda, R., S.L., H., Yang, R. K., et al. Relationship between 24-hour urinary-free cortisol excretion and salivary cortisol levels sampled from awakening to bedtime in healthy subjects. Life Sci (2003) 73(3):349-358. 


\section{References}

Zanetti, O., Frisoni, G. B., Bianchetti, A., et al. Depressive symptoms of Alzheimer caregivers are mainly due to personal rather than patient factors. Int J Geriatr Psychiatry (1998) 13(6):358-67.

Zannetti, O., Metitieri, A., Bianchetti, A., et al. Effectiveness of an educational program for demented person's relatives. Arch Gerontol Geriatr suppl (1998) 6:531-538.

Zarit, S. H. Behavioral disturbances of dementia and caregiver issues. International psychogeriatrics (1996) 8(3):263268.

Zarit, S. H., Anthony, C. R., and Boutselis, M. Interventions with care givers of dementia patients: comparison of two approaches. Psychol Aging (1987) 2(3):225-32.

Zubenko, G. S., Moossy, J., Martinez, A. J., et al. Neuropathological and neurochemical correlates of psychosis in primary dementia. Archives of Neurology (1991) 48:619-624. 


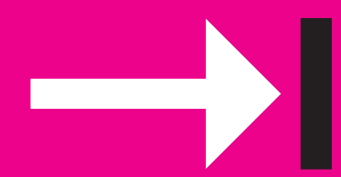

Dankwoord 
Met plezier kijk ik terug op mijn aio-periode. Promoveren was voor mij een interactief proces, waarin ik heb genoten van de prettige, enthousiasmerende en prikkelende samenwerking met vele anderen. Ik wil iedereen bedanken die op welke manier dan ook een bijdrage heeft geleverd aan dit proefschrift. Ik wil graag een aantal mensen persoonlijk bedanken, waarbij ik vrees voor mogelijke nalatigheden.

Ten eerste hebben de participanten van het MAASBED onderzoek een zeer essentiële bijdrage geleverd aan dit proefschrift. Ik dank hen hartelijk voor hun medewerking en openheid in het onderzoek, dat voor hen tijdsrovend en vaak confronterend was.

Mijn promotor, professor Frans Verhey. Beste Frans, bedankt voor het leerzame, inspirerende en gezellige 'maasbedje'. Je weet als geen ander de essentie uit zaken te halen en hebt oog voor wat zowel wetenschappelijk als klinisch relevant is. Ik ben je erkentelijk voor de goede begeleiding, waarbij je altijd openstond voor mijn mening.

Mijn tweede promotor, professor Jelle Jolles. Beste Jelle, bedankt dat ik heb mogen profiteren van je wetenschappelijke expertise. Ik ben je zeer erkentelijk dat je me de kans hebt gegeven me onder jouw vleugels niet alleen als wetenschapper, maar ook als clinicus te ontwikkelen.

Mijn copromotor, Fred Stevens. Beste Fred, ik heb veel geleerd en genoten van onze vele discussies. Je wist me steeds weer te enthousiasmeren en te inspireren. Bedankt dat je me door mijn 'kwalitatieve avontuur' hebt geloodst en dat je deur altijd voor mij open stond.

Richel, je was mijn statistische steun en toeverlaat. Bedankt voor je vele wijze lessen. En, al was ik blij om te merken dat ik je steeds minder nodig had, samen met jou data analyseren blijft toch het leukst! Niet alleen vanwege je enthousiasme, maar ook vanwege het feit dat dit vaak uitmondde in goede gesprekken over wetenschap en vele andere zaken. Ik heb je betrokkenheid en steun erg gewaardeerd.

Pauline, met jou deelde ik het aio-schap, een kamer, artikels, congressen, en nog veel meer. We hebben ons samen in het "Maasbed" ontwikkeld in de wetenschap. Bedankt voor de samenwerking, je steun, je grote bijdrage aan mijn proefschrift en het feit dat je het al die tijd met mij hebt uitgehouden. Ik hoop dat we ook van "Maasbed in de praktijk" een succes kunnen maken. 
Niek Jaspers, jij maakte het ons mogelijk het onderzoek ook op de RIAGG uit te voeren. Ik dank je zeer voor je feedback na het lezen van mijn artikels en je klinische blik. Ook de overige medewerkers van de afdeling ouderenzorg van de RIAGG Maastricht bedank ik voor hun medewerking bij het uitvoeren van het onderzoek. Jullie gaven mij altijd het gevoel zeer welkom te zijn op jullie afdeling. Met name Yvonne van Zutphen en Frank Amory dank ik voor de prettige samenwerking en betrokkenheid.

De onderzoeksassistenten Maartje Linzell, Esmeralda van Pol, leke Winkens en Birgit Senden dank ik voor het mee verzamelen van alle onderzoeksgegevens. Zonder jullie hulp waren wij nu nog bezig geweest met dataverzamelen. Birgit, jij hebt het grootste deel van de logistiek en patiëntbezoeken voor je rekening genomen. Dank voor je inzet, het goede werk en de gezelligheid. leke, je was voor mij een grote steun. Ik heb met een gerust hart de dataverzameling van de verzorgers aan jou uit handen gegeven. Bedankt dat je met humor en flexibiliteit zo enorm veel werk voor mij hebt verzet. Ik heb alle vertrouwen in jouw aio-carrière! De stagiaires Peggy Stuijts, Liesbeth van Osch en Silvia de Wilde, ik dank ook jullie voor de hulp bij het verzamelen van onderzoeksgegevens. Jullie wisten er samen op de 'buurkamer' altijd een gezellige boel van te maken.

Alle co-auteurs wil ik bedanken voor hun waardevolle bijdragen aan de artikelen, met name Nancy Nicolson en Adriaan Honig. Dr. J. Sulon (Universiteit van Luik) dank ik voor het analyseren van de cortisol samples. Jane Sykes dank ik voor het corrigeren van mijn Engels. Jacqueline Mourik, bedankt voor de prettige samenwerking en succes met de eindfase van jouw promotie-onderzoek. De leden van de beoordelingscommissie dank ik voor het beoordelen van het manuscript.

Veel collega's van de vakgroep Psychiatrie en Neuropsychologie hebben direct of indirect een bijlage geleverd aan dit proefschrift, mijn dank hiervoor. Een aantal van hen wil ik nog bij naam noemen. Ankie Hochstenbach, Laurent Louwies, Marco van Hertrooy en Nico Rozendaal, bedankt voor jullie ondersteuning. Kitty Verwoerdt, dank voor al je hulp, zeker ook in de laatste 'regel'fase. Rudolf Ponds, bedankt voor het superviseren van de neuropsychologische verslaglegging en je belangstelling. Geert Leenders, jij weet mij altijd weer te verrassen met een onuitputtelijke stroom van creatieve ideeën. Dankzij jou had ik het voorrecht mee te mogen werken aan een aantal zeer leuke projecten. En niet in de laatste plaats ben ik je zeer dankbaar voor het pr-werk dat je voor mij en mijn medeaio's hebt verricht. Mijn mede-aio's dank ik voor hun bijdrage aan de aio-bijeenkomsten en ook voor de vele gezellige avondjes, waarvan een aantal (Sint-)avondjes onvergetelijk zijn (met dank aan Ivo). Ivo, jij was vanaf het begin de kartrekker van onze aio-club. Ik heb 
je tevens leren waarderen als een goede gesprekspartner. Ik mis onze gesprekken over de wetenschap, de klinische praktijk en het leven, dus laten we maar weer eens een avondje plannen! Ook de gezellige avonden met collega's in de kroeg heb ik zeer kunnen waarderen. Jeroen Schmitt, op jou kon je dan altijd rekenen. Het feit dat je binnenkort vertrekt naar Zwitserland zal zeker ten koste gaan van de gezelligheid. Susan van Hooren, bedankt voor het feit dat ik altijd even bij je kon binnenlopen met vragen of verhalen; en ons tripje naar Genève was toch zeker een van de hoogtepunten (op het congres na). Ik dank ook mijn 'nieuwe' collega's in Vijverdal voor hun interesse tijdens de afronding van mijn proefschrift.

Een aantal vrienden verdienen hier een speciale plek.

Sascha Rasquin, lieve Sas. Jij was mijn collega en kamergenootje maar bent veel meer dan dat. Je was altijd geïnteresseerd en bereid te helpen. Ik bewonder je onuitputtelijke energie en je doorzettingsvermogen. Ik ben blij dat je me als paranimf wilt bijstaan en ik straks hetzelfde voor je terug mag doen.

Dorine Willemse, lieve Dorine. Wat hebben we een leuke tijd gehad samen in Maastricht! Het lijkt alweer zo lang geleden, vooral nu er voor jou een nieuwe levensfase is begonnen. Maar je hebt het begin van mijn "Maastrichtse carrière" meegemaakt en daarom vind ik het zo leuk dat je nu nog eens naar het zuiden afzakt om mijn paranimf te zijn.

Petra Hurks en Suzanne Valentijn, al staan jullie straks misschien niet letterlijk achter mij, toch voelt het wel zo. Lieve Petra, wellicht is een teken van goede vriendschap dat je uren met elkaar kunt doorbrengen zonder dat je uitgepraat raakt. Bij ons is dat zeer zeker het geval, tot vermoeienis van onze mede-treinreizigers. Bedankt voor de gezellige uurtjes in en buiten de trein en voor het feit dat je altijd voor me klaar staat. Lieve Suus, ook jij bent inmiddels meer dan een collega geworden en dat voelt heel vanzelfsprekend. We zitten in veel zaken op dezelfde lijn en dat maakt ons een goede match. Ik wil jou en Corne bedanken voor de vele logeerpartijtjes, waarvan er wellicht ook nog wel meer zullen volgen. En meiden ....op naar Barcelona!

Mijn vrienden buiten Maastricht, al noem ik jullie niet bij naam, mijn dank gaat evenzeer naar jullie uit. Ik ben blij dat we in onze drukke levens toch regelmatig tijd voor elkaar vrij weten te maken. Alle vrienden, veel dank voor jullie interesse, steun en gezelligheid.

Mijn familie en schoonfamilie, bedankt dat jullie er altijd voor me zijn en me bij alles steunen. Jullie belangstelling en begrip heb ik erg gewaardeerd. Lieve pap en mam, bedankt voor zoveel dingen, maar vooral voor jullie wijze adviezen, onvoorwaardelijke steun, liefde en vertrouwen. 
Lieve Alard, ik denk dat ik met recht kan zeggen dat dit proefschrift er zonder jou heel anders had uitgezien. Hoe kan ik je ooit bedanken voor die vele uren werk waarmee ik je heb opgezadeld. Jij wist mij als geen ander te motiveren in mijn werk: pas toen de doctors titel in zicht kwam, wilde je eindelijk met mij trouwen. Dat we nog maar lang samen mogen genieten van het leven! 
Dankwoord 


\section{Curriculum Vitace}


Marjolein de Vugt werd geboren op 17 augustus 1975 te Tilburg. In 1993 behaalde zij haar WWO diploma aan het Mill-Hillcollege te Goirle. Hierna ging zij aan de Universiteit Maastricht (UM) Gezondheidswetenschappen studeren, afstudeerrichting Geestelijke Gezondheidskunde (GGK). In 1995 startte zij tevens met de studie Psychologie, afstudeerrichting Biologische Psychologie. Tijdens het laatste jaar van haar studie werkte zij als student-assistent bij de vakgroep Psychiatrie en Neuropsychologie van de UM ten behoeve van een onderzoek naar Mild Cognitive Impairment. Na een onderzoeksstage op de Academische Afdeling Neuropsychologie van Psychomedisch Streekcentrum Vijverdal en een klinische stage op de Geheugenpolikliniek van het Academisch Ziekenhuis Maastricht (AZM), behaalde zij in 1998 voor beide studies haar diploma. Aansluitend werkte zij als neuropsycholoog op de Geheugenpolikliniek van het AZM. In 1999 werd zij als assistent in opleiding aangesteld bij de vakgroep Psychiatrie en Neuropsychologie van de UM waar zij werkte aan dit proefschrift. In januari 2003 is zij gestart met de postdoctorale opleiding tot Gezondheidszorgpsycholoog. In het kader van deze opleiding werkt zij momenteel op de afdeling Niet-Aangeboren Hersenletsel en de afdeling Ouderenzorg van Psychomedisch Streekcentrum Vijverdal te Maastricht. 